University of Nebraska - Lincoln

DigitalCommons@University of Nebraska - Lincoln

Faculty Publications, Department of Physics and Astronomy

Research Papers in Physics and Astronomy

2016

\title{
Electronic Structure of Iron Porphyrin Adsorbed to the Pt(111)
}

Surface

\author{
Daniel P. Miller \\ State University of New York at Buffalo \\ James Hooper \\ Jagiellonian University, Krakow \\ Scott Simpson \\ St. Bonaventure University \\ Paulo S. Costa \\ University of Nebraska - Lincoln, pcosta2@unl.edu \\ Nina Tyminska \\ State University of New York at Buffalo
}

See next page for additional authors

Follow this and additional works at: https://digitalcommons.unl.edu/physicsfacpub

Miller, Daniel P.; Hooper, James; Simpson, Scott; Costa, Paulo S.; Tyminska, Nina; McDonnell, Shannon M.; Bennett, Jason A.; Enders, Axel; and Zurek, Eva, "Electronic Structure of Iron Porphyrin Adsorbed to the Pt(111) Surface" (2016). Faculty Publications, Department of Physics and Astronomy. 194.

https://digitalcommons.unl.edu/physicsfacpub/194

This Article is brought to you for free and open access by the Research Papers in Physics and Astronomy at DigitalCommons@University of Nebraska - Lincoln. It has been accepted for inclusion in Faculty Publications, Department of Physics and Astronomy by an authorized administrator of DigitalCommons@University of Nebraska - Lincoln. 


\section{Authors}

Daniel P. Miller, James Hooper, Scott Simpson, Paulo S. Costa, Nina Tyminska, Shannon M. McDonnell, Jason A. Bennett, Axel Enders, and Eva Zurek 


\section{Electronic Structure of Iron Porphyrin Adsorbed to the Pt(111) Surface}

Daniel P. Miller, ${ }^{\dagger}$ J James Hooper, ${ }^{\ddagger}$ Scott Simpson, ${ }^{\text {IL }}$ Paulo S. Costa, ${ }^{\#}$ Nina Tymińska, ${ }^{\dagger}$ Shannon M. McDonnell, $\|$ Jason A. Bennett, ${ }^{*} \|^{\dagger}$ Axel Enders, ${ }^{\perp, \#}$ and Eva Zurek*, ${ }^{\dagger}$

${ }^{\dagger}$ Department of Chemistry, State University of New York at Buffalo, Buffalo, New York 14260-3000, United States

${ }^{\ddagger}$ Department of Theoretical Chemistry, Faculty of Chemistry, Jagiellonian University, 30-060 Krakow, Poland

${ }^{\mathbb{T}}$ Department of Chemistry, St. Bonaventure University, St. Bonaventure, New York 14778, United States

"School of Science, Penn State Behrend, Erie, Pennsylvania 16563, United States

${ }^{\perp}$ Experimentalphysik V, Universität Bayreuth, 95440 Bayreuth, Germany

\# Department of Physics and Astronomy, University of Nebraska-Lincoln, Lincoln, Nebraska 68588-0299, United States

\section{Supporting Information}

ABSTRACT: Systematic density functional theory calculations that treat the strong on-site $3 \mathrm{~d}$ electron-electron interactions on iron via a Hubbard $U_{\text {eff }}=3.0 \mathrm{eV}$ and the van der Waals $(\mathrm{vdW})$ interactions between the substrate and adsorbate within the vdW-DF framework are employed to study the adsorption of the iron porphyrin ( $\mathrm{FeP})$ molecule to the $\mathrm{Pt}(111)$ surface. The more accurate vdW-DF-optPBE and vdW-DF-optB88 functionals found the same binding site to be the most stable and yielded binding energies that were within $\sim 20 \%$ of each other, whereas the binding energies computed with the vdW-DF-revPBE functional were substantially weaker. This work highlights the importance of vdW interactions for organometallic molecules chemisorbed to transition metal surfaces. The stability of the binding sites was found to depend upon the number of $\mathrm{Fe}-\mathrm{Pt}$ and $\mathrm{C}-\mathrm{Pt}$ bonds that were formed. Whereas in the gas phase the most stable spin state of $\mathrm{FeP}$ is the intermediate spin $S=$ 1 state, the high spin $S=2$ state is preferred for the $\mathrm{FeP}-\mathrm{Pt}(111)$ system on the binding sites

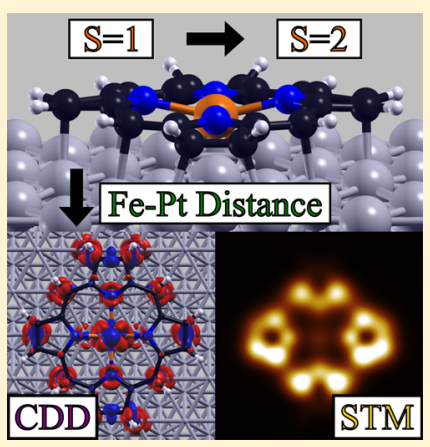
considered herein. The spin switch results from the elongation of the $\mathrm{Fe}-\mathrm{N}$ bonds that occur upon adsorption.

\section{INTRODUCTION}

The investigation, manipulation and exploitation of the magnetism of single atoms is of substantial interest, both from a basic science point of view as well as in the context of applications in information processing and storage. Experimentally, magnetic moment bearing transition metal centers with localized $d$ - and/or $f$-states are typically deposited on flat crystalline surfaces or template surfaces at low temperature using surface-science approaches. ${ }^{1}$ Given the high mobility of those atoms at room temperatures, it is of more practical use to stabilize them using organic host molecules, such as, for instance, in single molecular magnets, ${ }^{2}$ spin crossover complexes, ${ }^{3}$ metal-organic structure frameworks, ${ }^{4}$ and other organic-containing structures. Organic ligands or molecules create a ligand field around the magnetic center, which typically determines the spin state, magnetic moment, and the magnetic anisotropy. Metal-center containing molecules are also amenable to $2 \mathrm{D}$ and $3 \mathrm{D}$ self-assembly and can thus help to create long-range ordered arrays of magnetic centers. ${ }^{5}$

Porphyrin molecules have attracted particular interest as hosts for magnetic centers. Nearly all $3 \mathrm{~d}$ metals can be incorporated into the macrocycles, and they easily form noncovalent $2 \mathrm{D}$ assemblies on flat, crystalline surfaces, ${ }^{6-10}$ as well as $3 \mathrm{D}$ metal-organic frameworks. They have thus become a prototypical system for the study of coordination effects and molecular spintronics, as is evident by the large number of studies of metalated porphyrins on surfaces alone. ${ }^{6-9,11-25}$ The local conductivity of the molecules correlates with the spin state of the central metal atom, ${ }^{10,11,26,27}$ and local probes, specifically scanning tunneling microscopes (STM), can be used to detect and manipulate the local magnetic properties. ${ }^{8,26}$ Other effects conveniently observable on $2 \mathrm{D}$ porphyrin layers are adsorbate and substrate effects, such as superexchange between the metal centers through the substrate. ${ }^{11}$

The spin states of iron porphyrin $(\mathrm{FeP})$ molecules are intimately related to their oxidation state and geometry so they may be switched by external factors including ligation, temperature, pressure, electric fields, and light. In the ground state in the gas phase, the $\mathrm{Fe}(\mathrm{II})$ center within the iron porphyrin molecule assumes an intermediate spin (IS) state of $S=1$. Recently, it has been shown that the high spin (HS) state, $S=2$, can be attained via surface adsorption. For example, density functional theory (DFT) calculations predicted that the HS state will be preferred when iron porphyrins are physisorbed on $\mathrm{Au}(111),{ }^{9}$ chemisorbed to various magnetic substrates including $\mathrm{Co}(001), \mathrm{Ni}(001), \mathrm{Ni}(110)$, and $\mathrm{Ni}(111)$,

Received: September 17, 2016

Revised: November 21, 2016

Published: November 30, 2016 
or deposited at a divacancy site in a graphene lattice. ${ }^{28}$ However, DFT predicts that the $S=1$ state will be maintained upon adsorption to the $\mathrm{Cu}(001)$ surface. ${ }^{17}$ Moreover, DFT calculations have shown that the spin state of the related manganese(II) porphine molecule can be perturbed via adsorption to the $\mathrm{Au}(111)$ surface, and application of an electric field to this system also alters its magnetic properties. ${ }^{16}$

For the $\mathrm{FeP}$ molecule, a ground state configuration of $\left(\mathrm{d}_{x y}\right)^{2}\left(\mathrm{~d}_{\pi}\right)^{2}\left(\mathrm{~d}_{z}\right)^{2}$ has been found via Mössbauer, magnetic, and NMR measurements, ${ }^{29-34}$ whereas Raman spectroscopy suggests a $\left(\mathrm{d}_{x y}\right)^{2}\left(\mathrm{~d}_{\pi}\right)^{3}\left(\mathrm{~d}_{z}\right)^{1}$ configuration. ${ }^{35}$ Theoretical methods can also yield different occupations. ${ }^{36,37}$ Results from calculations that approach the accuracy of $\operatorname{CCSD}(\mathrm{T})$, which utilize complete basis set extrapolation, ${ }^{38}$ are in agreement with the Raman studies. The $\operatorname{CCSD}(\mathrm{T})$ approximation is the coupled cluster method that includes singles, doubles, and noniterative triples, i.e., singly-, doubly-, and triply excited determinants, where the triples correction to the energy is evaluated using exactly the same formula as in fourth-order perturbation theory. Important for this study is that the $\mathrm{d}_{x^{2}-y^{2}}$ based molecular orbital, which is antibonding with the nitrogen atoms, remains unoccupied in the ground state. A mechanical strain that leads to the elongation of the $\mathrm{Fe}-\mathrm{N}$ bond will therefore result in the lowering of the energy of this orbital. When the energy is low enough, the orbital occupation becomes $\left(\mathrm{d}_{x y}\right)^{2}\left(\mathrm{~d}_{\pi}\right)^{2}\left(\mathrm{~d}_{z^{2}}\right)^{1}\left(\mathrm{~d}_{x^{2}-y^{2}}\right)^{1}$, and the $S=2$ spin state is assumed. The spin switching that occurs upon surface adsorption is due to elongation of the $\mathrm{Fe}-\mathrm{N}$ bond, which may be a result of charge transfer between the molecule and the metal, epitaxial fit, or a structural distortion of the macrocyclic ring.

The rapidly growing number of mostly experimental studies on related systems calls for comprehensive, systematic, and reliable computational data. It has been shown that an accurate description of van der Waals (vdW) dispersion interactions is crucial in obtaining the correct binding energies of benzene chemisorbed to transition metal surfaces. ${ }^{39-42}$ Therefore, herein we study the surface-induced spin switching within the FeP molecule via DFT calculations that include dispersion interactions within the vdW-DF framework. ${ }^{43-46}$ In the context of adsorbate magnetism, Pt stands out as a substrate because it can easily acquire an induced magnetic moment, contribute to the magnetic anisotropy of magnetic adsorbate structures, and has a relatively large spin-orbit coupling; thus, it could be a component of organics-based spin hybrid systems. ${ }^{47-50}$ Our study investigates the adsorption of $\mathrm{FeP}$ to the $\mathrm{Pt}(111)$ surface in a systematic manner to establish the correlation between binding sites, geometry, and local electronic structure.

\section{COMPUTATIONAL METHODS}

Periodic DFT calculations were performed using the Vienna $A b$ initio Simulation Package (VASP) version 5.3.5. ${ }^{51}$ Each system was optimized with the vdW-DF-optPBE, ${ }^{43}$ vdW-DF-optB88, ${ }^{43}$ and the vdW-DF-revPBE ${ }^{52}$ van der Waals functionals, and the dispersion forces were accounted for within these frameworks. $^{44,45}$ These vdW-DF methodologies provide a better description of dispersion for surface-adsorbate systems over the standard generalized gradient approximation (GGA) functionals. ${ }^{46}$ Experimental estimates for the binding energy of benzene to the $\mathrm{Pt}(111)$ surface range from -34.4 to -44.0 $\mathrm{kcal} / \mathrm{mol}$ (see refs 39 and 40 and references within). It has been shown that the vdW-DF-optPBE and the vdW-DF-optB88 functionals perform the best for this system, with the former yielding smaller binding energies than the latter. ${ }^{39,40}$

The projector augmented wave (PAW) method $^{53}$ was used to treat the core states along with a plane-wave energy cutoff of $500 \mathrm{eV}$, and the $\mathrm{C} / \mathrm{N} / \mathrm{O} 2 \mathrm{~s} / 2 \mathrm{p}, \mathrm{H} 1 \mathrm{~s}, \mathrm{Fe} 4 \mathrm{~s} / 3 \mathrm{~d}$, and $\mathrm{Pt} 6 \mathrm{~s} /$ $5 \mathrm{~d}$ electrons were treated explicitly. Mass-velocity relativistic effects were included; however, spin orbit coupling was not considered because the computational cost involved is high and because it was not necessary to include spin orbit coupling to successfully theoretically study the adsorption of benzene to $\operatorname{Pt}(111) .^{39,40}$ A $\Gamma$-centered Monkhorst-Pack scheme ${ }^{54}$ was used to generate $k$-point grids, and a $5 \times 5 \times 1 k$-mesh provided total energies and binding energies converged to within 0.60 $\mathrm{kcal} / \mathrm{mol}$ per unit cell when compared to a $7 \times 7 \times 1 \mathrm{k}$-mesh.

The surface was simulated using a three layer slab containing $90 \mathrm{Pt}$ atoms, with a $\sim 30 \AA$ vacuum space between adjacent slabs. The iron porphyrin adsorbate and the top layer of the $\mathrm{Pt}(111)$ surface were allowed to relax fully, while the bottom two layers of the $\operatorname{Pt}(111)$ surface were kept fixed. The computed lattice constants of $4.00 \AA$ (vdW-DF-optPBE), 3.98 $\AA$ (vdW-DF-optB88), and $4.04 \AA$ (vdW-DF-revPBE) were used in the construction of the $\mathrm{Pt}(111)$ surface slabs. The number of molecules per surface area of the metal surface, i.e., the surface coverage, was approximately 0.48 molecules $/ \mathrm{nm}^{2}$.

The Hubbard $U$ term was used to describe the d-states in iron. ${ }^{55}$ We employed a $U_{\text {eff }}=U-J=3.0 \mathrm{eV}$, as it has been shown to accurately reproduce the electronic and magnetic structure of $\mathrm{FeP}$ in the gas phase. ${ }^{56,57}$ In addition, a $U_{\text {eff }}$ of $3.0 \mathrm{eV}$ has been successfully employed to study the adsorption of $\mathrm{Fe}$ (II) porphyrins to surfaces. ${ }^{7,17,28}$ Benchmark calculations showed that a $U_{\text {eff }}=3.0 \mathrm{eV}$ yielded the correct $S=1$ ground spin state for $\mathrm{FeP}$ for all of the functionals considered herein.

The Avogadro molecular editor ${ }^{58}$ was used for building and analyzing the structures, and the figures that illustrate the molecule adsorbed to the surface, the charge density difference plots, and the spin densities were prepared with the XCrySDen software. $^{59}$

\section{RESULTS AND DISCUSSION}

FeP in the Gas Phase. To get a better understanding of the electronic structure of the $\mathrm{FeP}$ molecule in the gas phase, we projected the $\mathrm{Fe} \mathrm{d}$-states and plotted the electron density associated within energy windows that encompassed these levels, as shown in Figure 1. In addition to containing a small amount of Fe d-character, the occupied peaks labeled as 1, 2, and 6 comprise primarily orbitals that stem from the porphyrin atoms. The regions $3,5,7,8,13$, and 15 are primarily of Fe dcharacter, whereas $4,9,10,11,12$, and 14 contain a substantial amount of metal and ligand character. Comparison of these charge densities with the molecular orbitals calculated using the molecular ADF package ${ }^{60,61}$ and the revPBE ${ }^{62}$ functional yielded good agreement. The gap between the highest occupied molecular orbital (HOMO) and the lowest unoccupied molecular orbital (LUMO) was $0.7 \mathrm{eV}$ with the molecular code vs $1.4 \mathrm{eV}$ with the periodic program. The reason for the larger gap obtained with the latter is because a Hubbard $U$, which opens up band gaps, was used in the periodic calculations. Both the molecular and periodic calculations indicate that the $S=1$ state has the $\left(\mathrm{d}_{x y}\right)^{2}\left(\mathrm{~d}_{\pi}\right)^{3}\left(\mathrm{~d}_{z}\right)^{1}$ configuration, in agreement with computations that approach the accuracy of $\operatorname{CCSD}(\mathrm{T})^{38}$ and with Raman studies. ${ }^{35}$ A similar plot for the less stable $S=2$ state is provided in the SI. 
$\mathrm{FeP} S=1$

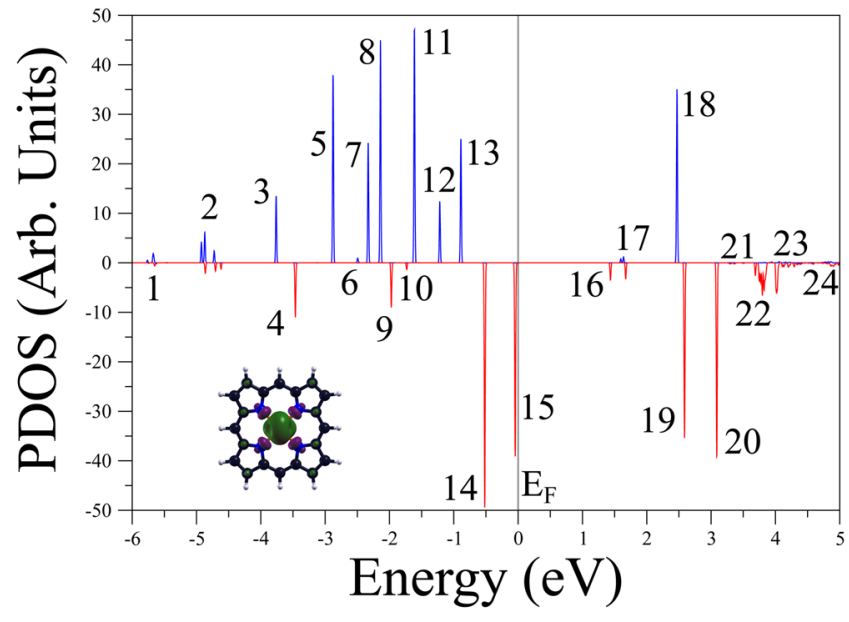

1

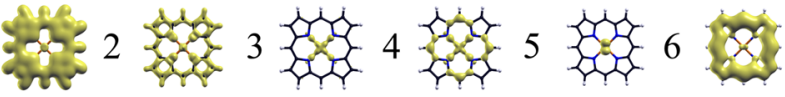

7

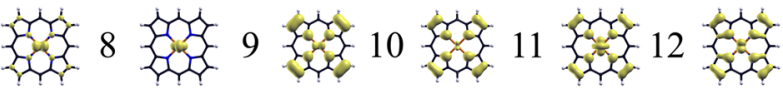

13
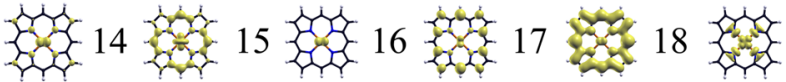

19
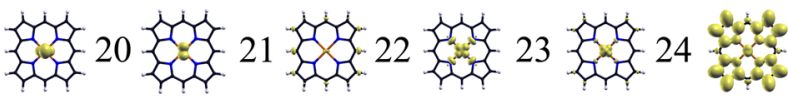

Figure 1. Projected densities of states (PDOS) for the Fe d-states in the isolated $S=1 \mathrm{FeP}$ molecule, coupled with isosurfaces of partial charge densities for the various peaks (isovalues $=0.01-0.1 \mathrm{e} / \AA^{3}$ ) calculated with the vdW-DF-optPBE functional. An isosurface of the spin density (isovalue $= \pm 0.01 \mathrm{e} / \AA^{3}$ ) is given in the inset.

Adsorption of FeP to the $\mathrm{Pt}(111)$ Surface. In order to find the most stable binding site, the geometries of eight high symmetry configurations of $\mathrm{FeP}$ adsorbed to the $\mathrm{Pt}(111)$ surface were optimized. Figure 2 shows the unique binding sites considered in this study. The $\mathrm{Fe}$ atom can be centered on a hollow hexagonally close packed $\left(\mathrm{H}_{\text {hcp }}\right)$ site, a hollow face

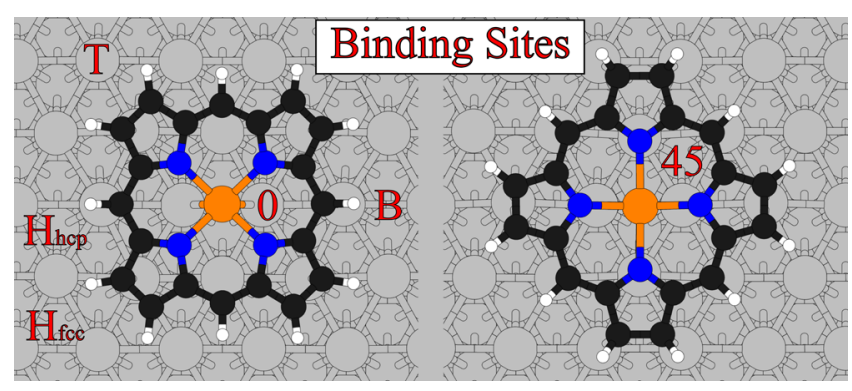

Figure 2. Binding sites considered for $\mathrm{FeP}$ adsorption to the $\mathrm{Pt}(111)$ surface. The Fe atom can lie on top of a bridge (B) binding site, a top (T) binding site, a hollow-hcp $\left(\mathrm{H}_{\mathrm{hcp}}\right)$ binding site, or a hollow-fcc $\left(\mathrm{H}_{\mathrm{fcc}}\right)$ binding site in the 0-configuration (left) or the 45-configuration (right). The difference between the two configurations is a $45^{\circ}$ rotation of the molecule about the $z$-axis. The white/black/blue/ orange/gray colored balls represent the hydrogen/carbon/nitrogen/ iron/platinum atoms, respectively. centered cubic $\left(\mathrm{H}_{\mathrm{fcc}}\right)$ site, a bridge (B) site, or a top $(\mathrm{T})$ site. In addition, the porphyrin ring can be rotated by $45^{\circ}$ with respect to the [100] lattice plane of the surface. The binding energies, select geometric parameters, and spin states of the optimized systems are provided in Tables 1, 2, and 3 for the vdW-DFoptPBE, vdW-DF-optB88, and vdW-DF-revPBE functionals, respectively. The $\mathrm{Fe}-\mathrm{Pt}$ bond lengths are also provided in these tables, as are the shortest and longest $\mathrm{C}-\mathrm{Pt}$ bonds found in each system. For comparison, the most stable adsorption site of benzene to $\mathrm{Pt}(111)$ is the $\mathrm{B}_{30}$ site with the distance between the carbon atoms and the metal surface measuring 2.1-2.2 $\AA$ with the functionals used herein. ${ }^{39,40}$ Benzene undergoes a Kekule distortion upon adsorption to $\mathrm{Pt}(111)$ with the hydrogen atoms puckering away from the surface. ${ }^{39}$

The most favorable binding site for adsorption of $\mathrm{FeP}$ to the $\mathrm{Pt}(111)$ surface was the $\mathrm{B}_{45}$ site for the vdW-DF-optPBE and vdW-DF-optB88 functionals, as shown in Figure 3, while the preferred binding site for vdW-DF-revPBE was the $\mathrm{H}_{\text {hcp-45, }}$, see the SI. In all cases, the high-spin $S=2$ state was the lowest in energy. The binding energy of the most stable site, computed with respect to the optimized $S=1$ state, was -105.13/ $-119.48 /-66.27 \mathrm{kcal} / \mathrm{mol}$ for the vdW-DF-optPBE/vdW-DFoptB88/vdW-DF-revPBE functionals, respectively. The trend in the strength of adsorption illustrates the well-known trends of these functionals, with vdW-DF-revPBE being the most repulsive functional and vdW-DF-optB88 being the most attractive functional. ${ }^{40,63}$ For the sites considered herein, the corrugation energies were calculated as being 27.48/32.71/ $24.14 \mathrm{kcal} / \mathrm{mol}$ for the aforementioned functionals. The large corrugation energy and short substrate-adsorbate distances are indicative of chemisorption. It has been shown that the binding energies of benzene to the $\mathrm{Pt}(111)$ surface calculated with the vdW-DF-optPBE and vdW-DF-optB88 functionals give the best agreement with experiment. ${ }^{39,40}$ Because the porphyrin ring is planar and aromatic with $\mathrm{sp}^{2}$ hybridized carbon atoms like benzene, it is likely that these two functionals will provide the most accurate results for the $\mathrm{FeP}$ system. We focus on analyzing the results for the vdW-DF-optPBE functional, whereas the results for the vdW-DF-optB88 functional are given in parentheses to highlight the similarities.

In the most stable $\mathrm{B}_{45}$ binding site, the $\mathrm{C}-\mathrm{Pt}$ distances of the hydrogenated carbons ranged from 2.18 to $2.60 \AA$ (vdW-DFoptB88: $2.17-2.60 \AA$ ). The $\mathrm{C}-\mathrm{Pt}$ distances of the carbon atoms bound to nitrogen were longer, measuring 2.75-2.91 $\AA$ (2.74-2.89 $\AA$ ). The hydrogen atoms bonded to those carbons that are closest to the surface pucker away from the surface, similar to what is found for adsorbed benzene because these carbons become $\mathrm{sp}^{3}$ hybridized upon coordination to the platinum surface. To calculate the charge density difference (CDD), the charge density of the $\mathrm{FeP}$ molecule and the surface (in the geometry of the optimized $\mathrm{FeP} / \mathrm{Pt}(111)$ system) was subtracted from the charge density of the full system. The CDD plots shown in Figure 4 illustrate a loss of charge directly above and beneath the platinum atoms that interact most strongly with the adsorbate because charge flows from the surface into the $\mathrm{FeP}-\mathrm{Pt}$ bonds. This electronic reorganization results in a buildup of charge roughly midway between the hydrogenated carbon atoms and the platinum atoms closest to them, indicative of chemical bond formation. The CDD also shows elements indicative of the $\mathrm{sp}^{2} \rightarrow \mathrm{sp}^{3}$ rehybridization of the carbon atoms that bind to the surface. Very little charge reorganization is seen between the nitrogen atoms and the surface, as well as between the carbons bonded to nitrogen and 
Table 1. Binding Energies (BE), Relative Energies, Spin State and Select Geometric Parameters of FeP Adsorbed to Pt(111) as Calculated with the vdW-DF-optPBE Functional for Various Binding Sites ${ }^{a}$

\begin{tabular}{|c|c|c|c|c|c|c|}
\hline site & $B E(\mathrm{kcal} / \mathrm{mol})$ & $\mathrm{Fe}-\mathrm{Pt}(\AA)$ & $\mathrm{C}-\mathrm{Pt}(\AA)$ & $\mathrm{N}-\mathrm{Pt}(\AA)$ & $S$ & $\mathrm{Fe}-\mathrm{N}$ bond length $(\AA)$ \\
\hline $\mathrm{B}_{45}$ & $-105.13(0.00)$ & $2.69,2.72(2)$ & $2.18-2.27(6)$ & 2.50 & 2 & 2.16 \\
\hline $\mathrm{H}_{\mathrm{fcc}-45}$ & $-101.29(3.84)$ & $2.67,2.68(2)$ & $2.19-2.28(4)$ & 2.63 & 2 & 2.15 \\
\hline $\mathrm{H}_{\text {hcp }-45}$ & $-96.32(8.81)$ & $2.78,2.78(2)$ & $2.18-2.30(4)$ & 2.53 & 2 & 2.12 \\
\hline $\mathrm{B}_{45}-\mathrm{IS}$ & $-94.93(10.20)$ & $2.88,2.90(2)$ & $2.18-2.28(6)$ & 2.50 & 1 & 2.09 \\
\hline $\mathrm{T}_{0}$ & $-89.62(15.51)$ & $2.54(1)$ & $2.15-2.28(10)$ & 2.60 & 2 & 2.16 \\
\hline $\mathrm{H}_{\mathrm{fcc}-0}$ & $-89.59(15.54)$ & $2.65(1)$ & $2.19-2.26(3)$ & 2.86 & 2 & 2.13 \\
\hline $\mathrm{H}_{\mathrm{hcp}-0}$ & $-88.51(16.62)$ & $2.76(1)$ & $2.22-2.26(3)$ & 2.91 & 2 & 2.12 \\
\hline $\mathrm{T}_{45}$ & $-78.45(26.68)$ & $2.50(1)$ & $2.24-2.26(4)$ & 2.75 & 2 & 2.06 \\
\hline $\mathrm{B}_{0}$ & $-77.65(27.48)$ & $2.72,2.73(2)$ & $2.23-2.24(2)$ & 2.87 & 2 & 2.12 \\
\hline
\end{tabular}

${ }^{a_{T}}$ The energies relative to the most stable binding site are given in parentheses. The shortest $\mathrm{Fe}-\mathrm{Pt}$ distances are provided with the number in the brackets corresponding to the number of these contacts. Ranges of $\mathrm{C}-\mathrm{Pt}$ distances that likely correspond to bonds are provided with the number in the brackets corresponding to the number of these contacts. The distances between the surface and the $\mathrm{N}$ atoms were obtained by subtracting the average of the atomic coordinates in the $z$-direction for the atoms in question minus an average of the $z$-coordinate of the atoms in the top layer of the $\mathrm{Pt}(111)$ surface. The spin state, $S$, and the average $\mathrm{Fe}-\mathrm{N}$ bond lengths are provided. The $S=1$ spin state for the $\mathrm{B}_{45}$ binding site is given as $\mathrm{B}_{45}-$ IS (intermediate spin).

Table 2. Same as Table 1 but for the vdW-DF-optB88 Functional

\begin{tabular}{|c|c|c|c|c|c|c|}
\hline site & $B E(\mathrm{kcal} / \mathrm{mol})$ & $\mathrm{Fe}-\mathrm{Pt}(\AA)$ & $\mathrm{C}-\mathrm{Pt}(\AA)$ & $\mathrm{N}-\mathrm{Pt}(\AA)$ & Fe spin & Fe-N bond length $(\AA)$ \\
\hline $\mathrm{B}_{45}$ & $-119.48(0.00)$ & $2.64,2.68(2)$ & $2.17-2.33(10)$ & 2.48 & 2 & 2.16 \\
\hline $\mathrm{T}_{0}$ & $-113.89(5.59)$ & $2.47(1)$ & $2.14-2.31(12)$ & 2.45 & 2 & 2.15 \\
\hline $\mathrm{H}_{\mathrm{fcc}-45}$ & $-111.65(7.83)$ & $2.63,2.65(2)$ & $2.18-2.33(8)$ & 2.51 & 2 & 2.15 \\
\hline $\mathrm{B}_{45}-\mathrm{IS}$ & $-111.32(8.16)$ & $2.84,2.88(2)$ & $2.17-2.35(10)$ & 2.47 & 1 & 2.08 \\
\hline $\mathrm{H}_{\mathrm{hcp}-45}$ & $-109.24(10.24)$ & $2.64,2.65(2)$ & $2.18-2.32(8)$ & 2.52 & 2 & 2.15 \\
\hline $\mathrm{H}_{\mathrm{hcp}-0}$ & $-95.77(23.71)$ & $2.63,2.81(2)$ & $2.19-2.32(4)$ & 2.63 & 2 & 2.11 \\
\hline $\mathrm{H}_{\mathrm{fcc}-0}$ & $-91.18(28.30)$ & $2.66,2.82,2.82(3)$ & $2.16-2.30(5)$ & 2.38 & 2 & 2.10 \\
\hline $\mathrm{B}_{0}$ & $-88.28(31.20)$ & $2.69,2.73(2)$ & $2.20-2.29(6)$ & 2.86 & 2 & 2.11 \\
\hline $\mathrm{T}_{45}$ & $-86.77(32.71)$ & $2.50(1)$ & $2.22-2.24(4)$ & 2.75 & 2 & 2.06 \\
\hline
\end{tabular}

Table 3. Same as Table 1 but for the vdW-DF-revPBE Functional ${ }^{a}$

\begin{tabular}{|c|c|c|c|c|c|c|}
\hline site & $B E(\mathrm{kcal} / \mathrm{mol})$ & $\mathrm{Fe}-\mathrm{Pt}(\AA)$ & $\mathrm{C}-\mathrm{Pt}(\AA)$ & $\mathrm{N}-\mathrm{Pt}(\AA)$ & Fe spin & $\mathrm{Fe}-\mathrm{N}$ bond length $(\AA)$ \\
\hline $\mathrm{H}_{\mathrm{hcp}-45}$ & $-66.27(0.00)$ & $2.76,2.78,3.20(3)$ & $2.18-2.31(3)$ & 2.94 & 2 & 2.16 \\
\hline $\mathrm{H}_{\mathrm{fcc}-45}$ & $-65.64(0.63)$ & $2.82,2.84,3.15(3)$ & $2.21-2.31$ & 2.88 & 2 & 2.16 \\
\hline $\mathrm{H}_{\mathrm{fcc}-0}$ & $-62.18(4.09)$ & $2.87,3.14,3.19(3)$ & $2.23-2.32(3)$ & 3.02 & 2 & 2.12 \\
\hline $\mathrm{H}_{\mathrm{hcp}-0}$ & $-61.64(4.63)$ & $2.98,3 \cdot 17,3 \cdot 22(3)$ & $2.24-2.33(3)$ & 3.06 & 2 & 2.12 \\
\hline $\mathrm{H}_{\mathrm{hcp}-45^{\mathrm{F}}}$-IS & $-60.72(5.55)$ & $3.27,3.29(2)$ & $2.17-2.32(3)$ & 3.03 & 1 & 2.04 \\
\hline $\mathrm{B}_{45}$ & $-60.48(5.79)$ & $2.72,2.79(2)$ & $2.20(4)$ & 2.62 & 2 & 2.16 \\
\hline $\mathrm{T}_{0}$ & $-56.23(10.04)$ & $2.59(1)$ & $2.16-2.34(4)$ & 2.98 & 2 & 2.10 \\
\hline $\mathrm{T}_{45}$ & $-55.41(10.86)$ & $2.61(1)$ & $2.40-2.41(2)$ & 3.16 & 2 & 2.10 \\
\hline $\mathrm{B}_{0}$ & $-42.13(24.14)$ & $2.92,2.95(2)$ & $2.26-2.27(2)$ & 2.96 & 2 & 2.11 \\
\hline
\end{tabular}

${ }^{a}$ The $S=1$ spin state for the $\mathrm{H}_{\mathrm{hcp}-45}$ binding site is given as $\mathrm{H}_{\mathrm{hcp}-45}$-IS.

the surface. If these carbon atoms were to form a chemical bond with the surface, one of the other atoms to which they are bonded would need to move away from the surface because of the change in hybridization, leading to substantial distortion within the porphyrin ring. Nitrogen loses charge in its lone pair, but there is a gain of charge along the $\mathrm{Fe}-\mathrm{N}$ bond.

Whereas the shortest distance between the $\mathrm{Fe}$ and $\mathrm{Pt}$ atoms, 2.69 and $2.72 \AA$ ( 2.64 and $2.68 \AA$ ), are indicative of bonding, the N-Pt distances, $2.48-2.81 \AA(2.48-2.78 \AA)$, are too long for bond formation. This is in stark contrast to the results computed for the interaction of FeP with the (001), (110), and (111) surfaces of $\mathrm{Co}$ and $\mathrm{Ni}$ where the formation of nitrogensurface bonds was found to be crucial for dictating the stability of the binding sites considered. ${ }^{7}$ The reason for this difference is that the atomic radius of platinum is approximately $0.3 \AA$ larger than that of cobalt and nickel, resulting in a longer metal-metal bond and concomitantly a larger distance between the substrate and the nitrogen atoms. The $\mathrm{Fe}$ atom in this system dips closer to the surface so that the $\mathrm{C}-\mathrm{C}-\mathrm{N}-\mathrm{Fe}$ dihedral (for the carbon and nitrogen atoms in the pyrrole-like ring) is $158-166^{\circ}\left(157-166^{\circ}\right)$. The CDD plots clearly show a gain of charge between the $\mathrm{Fe}$ and the two $\mathrm{Pt}$ atoms that it straddles, indicative of chemical bond formation, and charge loss in orbitals that have $\mathrm{Fe}$ d-character.

The $\mathrm{Fe}-\mathrm{N}$ distances in the molecule in the gas phase were calculated to be $2.01 \AA(2.00 \AA)$. Upon adsorption to the most stable binding site, they stretch to $2.11,2.11,2.19$, and $2.24 \AA$ (2.11, 2.11, 2.19, and $2.21 \AA$ ), as shown in Figure 3 . Calculations wherein the $\mathrm{Fe}-\mathrm{N}$ bonds were uniformly stretched in the gas phase FeP (but the rest of the molecule and the spin state were allowed to optimize) showed that at a distance of $2.18 \AA$, an $S=2$ state is more stable than an $S=0$ or $S=1$ state even with the pure GGA revPBE functional, which has a strong preference for the $S=1$ ground electronic state. 


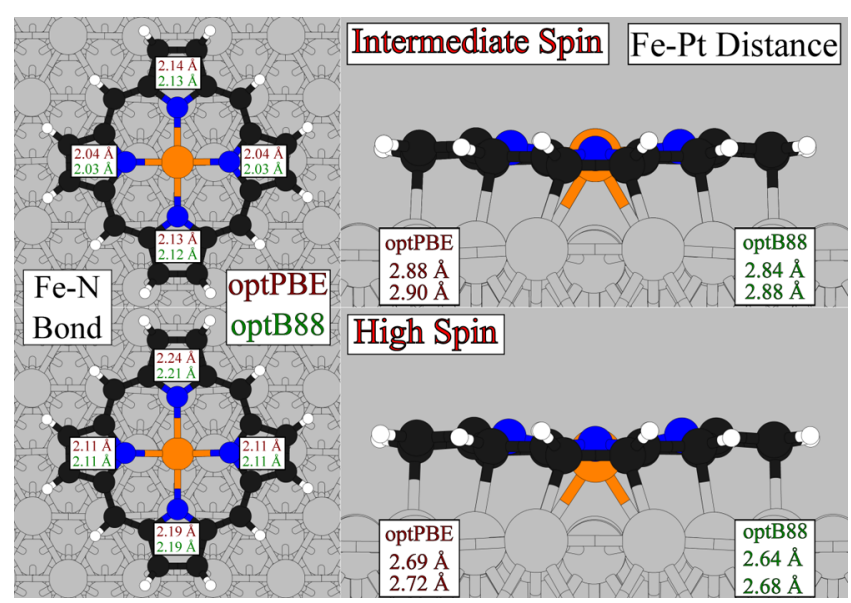

Figure 3. Top-view and side-view of the most stable adsorption geometries $\left(\mathrm{B}_{45}\right.$ site $)$ of $\mathrm{FeP}$ on the $\mathrm{Pt}(111)$ surface for the intermediate, $S=1$, and high, $S=2$, spin states obtained with the vdW-DF-optPBE and vdW-DF-optB88 functionals. The $\mathrm{Fe}-\mathrm{N}$ and $\mathrm{Fe}-\mathrm{Pt}$ distances are provided.

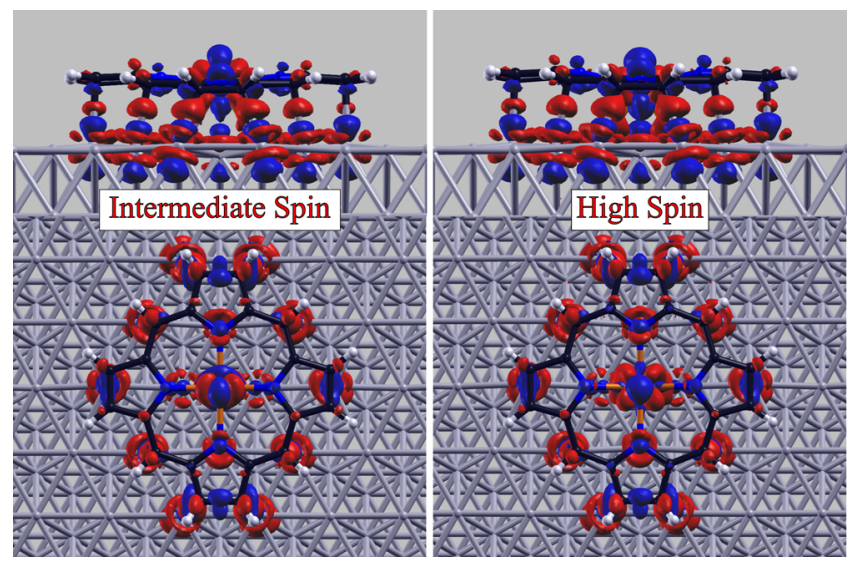

Figure 4. Isosurfaces of the charge density difference (CDD) for the $\mathrm{B}_{45}$ site were produced by subtracting the charge density of the adsorbate and the surface, in their distorted geometries, from the charge density of the interacting system. The distorted FeP molecule had the same spin as the one accrued on the surface. The CDDs are shown for the most stable FeP-Pt(111) structures found for the vdWDF-optPBE functional. The isovalue was set to $\pm 0.04 e / \AA^{3}$ and the $\mathrm{red} / \mathrm{blue}$ isosurfaces represent a gain/loss of electronic charge density.

We also optimized an intermediate $S=1$ spin state at the most stable binding site. This system was $10.20 \mathrm{kcal} / \mathrm{mol}(8.16 \mathrm{kcal} /$ mol) less stable than the preferred $S=2$ state. The main difference between its geometry and that of the high spin configuration is that it has longer $\mathrm{Fe}-\mathrm{Pt}$ distances of 2.88 and $2.90 \AA$ ( 2.84 and $2.88 \AA$ ). Because the iron atom did not pucker down toward the surface $\left(\mathrm{C}-\mathrm{C}-\mathrm{N}-\mathrm{Fe}\right.$ dihedrals of $168-172^{\circ}$ $\left.\left(167-173^{\circ}\right)\right)$, the porphyrin molecule remained relatively flat, but the $\mathrm{Fe}-\mathrm{N}$ bonds still elongated to 2.04, 2.04, 2.13, and $2.14 \AA(2.03,2.03,2.12$, and $2.13 \AA)$. Single-point calculations were carried out on the distorted $\mathrm{FeP}$ geometries from the $\mathrm{FeP} / \mathrm{Pt}(111)$ systems wherein the spin state was varied. As illustrated in Table 4, the most stable electronic configurations of the gas phase systems were the same as those on the surface. In the SI, we show that the increase in the $\mathrm{Fe}-\mathrm{N}$ bond distance and the preference for the $S=2$ state occurs even for a simple model system when a neutral ligand binds to the FeP molecule at one of the open coordination sites on Fe. Thus, the switch of
Table 4. Energies Relative to the Most Stable Spin State of the FeP Molecule Optimized in the Gas Phase, and the FeP Molecule in the Geometry It Attained at the $B_{45}$ Site on the $\operatorname{Pt}(111)$ Surface in the $S=1$ and $S=2$ Spin States

\begin{tabular}{|c|c|c|c|}
\hline system & $\begin{array}{c}S=0 \\
(\mathrm{kcal} / \mathrm{mol})\end{array}$ & $\begin{array}{c}S=1 \\
(\mathrm{kcal} / \mathrm{mol})\end{array}$ & $\begin{array}{c}S=2 \\
(\mathrm{kcal} / \mathrm{mol})\end{array}$ \\
\hline gas phase $\mathrm{FeP}$ & 12.2 & 0 & 2.6 \\
\hline $\begin{array}{l}\text { FeP from } \mathrm{FeP}-\mathrm{Pt}(111) \text { with } \\
\quad S=1\end{array}$ & 21.8 & 0 & 4.3 \\
\hline $\begin{array}{l}\text { FeP from } \mathrm{FeP}-\mathrm{Pt}(111) \text { with } \\
\quad S=2\end{array}$ & 28.9 & 16.1 & 0 \\
\hline
\end{tabular}

the spin state of the FeP molecule upon adsorption is a result of the geometric distortion it undergoes upon adsorption, and in particular by the elongation of the $\mathrm{Fe}-\mathrm{N}$ bonds. Moreover, it may be that this system is susceptible to the surface trans effect where the substrate, $\mathrm{Pt}(111)$ in this case, acts as an axial ligand. $^{64}$

The CDD of the intermediate and high spin states are qualitatively similar around all of the atoms with the exception of the iron atom. In the SI, a contour plot of the CDD that bisects the Fe atom shows that for the $S=1$ and $S=2$ systems there is charge gain in the region between the two surface $\mathrm{Pt}$ atoms and the $\mathrm{Fe}$ atom, but the region directly below the $\mathrm{Fe}$ atom loses charge density. The amount of charge accumulated in the $\mathrm{Fe}-\mathrm{Pt}$ bonds is larger in the $S=2$ system, but in the $S=1$ state charge is gained above the $\mathrm{Fe}$ atom. Closer inspection of the CDDs suggests that charge is lost in the $\mathrm{Fe}_{z^{2}}$ orbital upon adsorption in both systems. For the HS state, charge accumulates in a Fe $\mathrm{d}_{x y}$ orbital, and in the IS state, the gain occurs in an orbital of $\mathrm{d}_{\pi}$ symmetry. In the coordinate system used the Fe $d_{x^{2}-y^{2}}$ orbital points towards the nitrogen atoms. It should be pointed out, however, that the CDDs were obtained using the charge density of the FeP geometry attained on the surface whose spin state had the lowest energy (see Table 4).

The $S=2$ state was obtained for the geometry optimizations carried out on all of the binding sites considered, which further suggests that the $\operatorname{Pt}(111)$ substrate stabilizes the high spin system. Table 1 shows that for the $S=2$ state, the most stable binding sites have two $\mathrm{Fe}-\mathrm{Pt}$ bonds and between four and six $\mathrm{C}-\mathrm{Pt}$ bonds. Geometries that result in only one $\mathrm{Fe}-\mathrm{Pt}$ bond are generally less stable, with the system that has ten $\mathrm{C}-\mathrm{Pt}$ bonds being the most stable of these. When listed according to decreasing binding energy, the trends in the binding sites were similar, but not identical, for the vdW-DF-optPBE and vdWDF-optB88 functionals. However, in both cases the binding site preference was governed by the number of $\mathrm{Fe}-\mathrm{Pt}$ and $\mathrm{C}-\mathrm{Pt}$ bonds formed. In general the geometries optimized with the vdW-DF-optB88 functional resulted in a larger number of bonds between the surface and the substrate, consistent with the larger magnitude of the binding energies. For example, there are ten $\mathrm{C}-\mathrm{Pt}$ distances that fall between 2.16 and $2.33 \AA$ for the vdW-DF-optB88 functional at the $B_{45}$ site, but only six $\mathrm{C}-\mathrm{Pt}$ distances that are shorter than $2.27 \AA$ for vdW-DFoptPBE. The increased number of $\mathrm{C}-\mathrm{Pt}$ bonds at some of the binding sites as found with the vdW-DF-optB88 functional changed their order of stability as compared to what was found for the vdW-DF-optPBE functional. For the high-spin state, the $\mathrm{Fe}-\mathrm{N}$ bond stretched for all of the binding sites considered. The elongation was smallest at the $\mathrm{T}_{45}$ site with both vdW-DFoptPBE and vdW-DF-optB88, and for the former functional we 


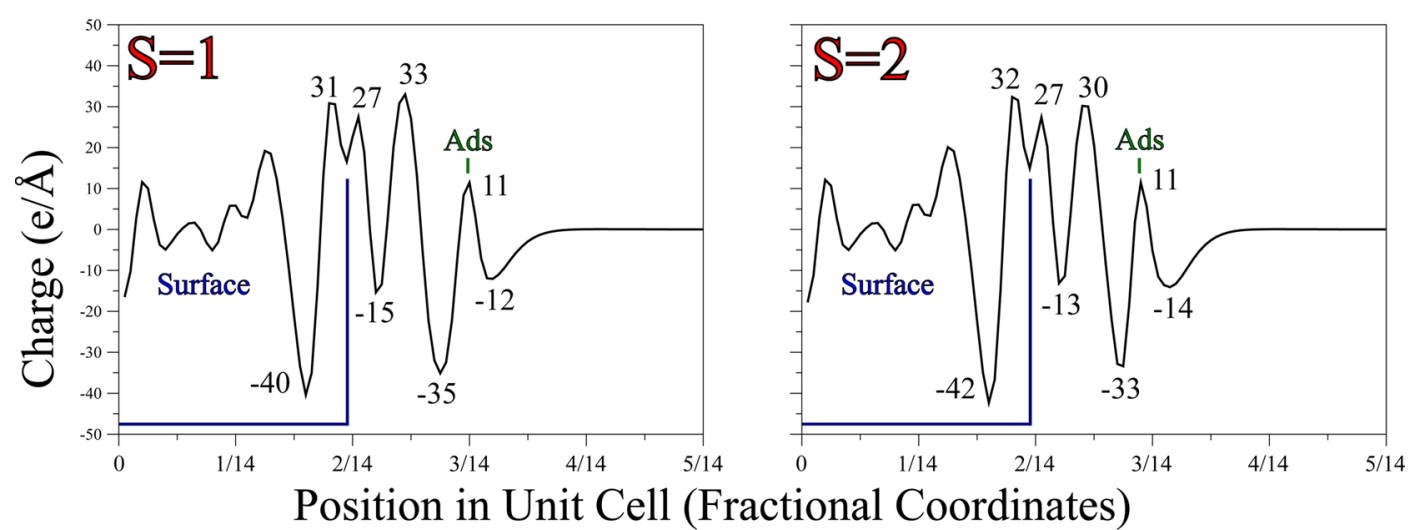

Figure 5. Plane averaged charge density difference taken along the $z$-axis as calculated with the vdW-DF-optPBE functional for the intermediate and high spin states of $\mathrm{FeP}$ adsorbed to the $\mathrm{Pt}(111)$ surface at the $\mathrm{B}_{45}$ site. The position of the porphyrin ring is schematically labeled as "Ads". Note that in the $S=2$ state the Fe atom puckers toward the surface, so it lies below the "Ads" marker.
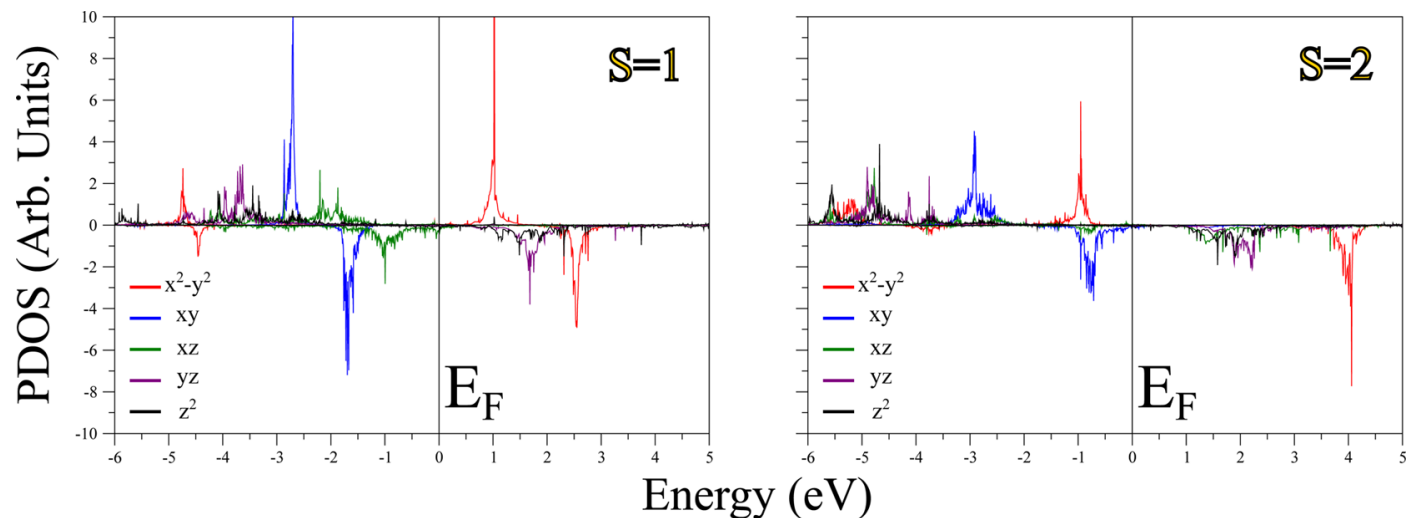

Figure 6. PDOS for the $d$-states in the intermediate $(S=1)$ and high spin $(S=2)$ state of FeP adsorbed to the $\mathrm{Pt}(111)$ surface at the $\mathrm{B}_{45}$ site as calculated with the vdW-DF-optPBE functional. The coordinate system was chosen so that the $\mathrm{Fe}_{x^{2}-y^{2}}$ orbitals pointed toward the nitrogen atoms on the porphyrin.

found an $S=1$ state at this site that was only slightly less stable in energy.

For the vdW-DF-revPBE functional, the high-spin state was the most stable at all of the binding sites considered. The magnitude of the binding energies were smaller than for the other two functionals, the $\mathrm{Fe}-\mathrm{Pt}$ distances were longer, and in general, there were fewer $\mathrm{C}-\mathrm{Pt}$ bonds at a given binding site. The optimized geometry at the most stable binding site is illustrated in the SI. The molecule is tilted so that only one side binds to the surface. Two $\mathrm{Fe}-\mathrm{Pt}$ bonds are formed, and only three carbons are close enough to interact with platinum. The average $\mathrm{N}-\mathrm{Pt}$ distance is almost half an angström longer than for the other functionals. The geometry is consistent with the smaller binding energy and corrugation energy. The $S=1$ state is $5.55 \mathrm{kcal} / \mathrm{mol}$ less stable than the high spin state. Based upon the previous benchmark calculations on the adsorption of benzene to $\operatorname{Pt}(111),{ }^{39,40}$ it is likely that the results obtained with the vdW-DF-revPBE functional are less accurate than with vdW-DF-optPBE and vdW-DF-optB88.

A Bader analysis was carried out to determine the magnitude and direction of charge transfer between the substrate and the adsorbate (see the SI). For the intermediate spin state, 0.28 e were transferred from the molecule to the surface. The close proximity of the iron atom to the platinum atoms in the high spin state resulted in a somewhat larger amount of molecule $\rightarrow$ surface charge transfer, 0.34e. The plane averaged CDD plots in Figure 5 show that the maximum charge lost per angström in the region directly below the molecular plane is similar for the high-spin geometry and the intermediate-spin geometry, $-33 e / \AA$ vs $-35 e / \AA$, as is the charge gained above the molecular plane, $11 e / \AA$. Moving closer to the surface, we see a region of charge gain between the substrate and adsorbate consistent with $\mathrm{Fe}-\mathrm{Pt}$ and $\mathrm{C}-\mathrm{Pt}$ bond formation for the $S=2$ and $S=1$ systems (30 and $33 e / \AA)$, followed by a region of charge loss $(-13$ and $-15 e / \AA)$ and then gain $(27-32$ and $27-$ $31 e / \AA ̊)$. The surface gains electronic charge, consistent with the Bader charge analysis.

To determine how the electronic structure of the gas phase $S=1$ ground state changes upon adsorption to the surface, the $d$-states for the $\mathrm{Fe}$ atom for the IS and HS states were projected, as shown in Figure 6. It was found that $S=1$ surface adsorption did not affect which orbitals were occupied so the electronic configuration remained as $\left(\mathrm{d}_{x y}\right)^{2}\left(\mathrm{~d}_{\pi}\right)^{3}\left(\mathrm{~d}_{z}\right)^{2}$. For the more stable $S=2$ spin state on the $\operatorname{Pt}(111)$ surface the occupied iron orbitals were determined to be the same as one would obtain for the gas phase system, $\left(\mathrm{d}_{x y}\right)^{2}\left(\mathrm{~d}_{\pi}\right)^{2}\left(\mathrm{~d}_{z^{2}}\right)^{1}\left(\mathrm{~d}_{x^{2}-y^{2}}\right)^{1}$. Because the $d_{x^{2}-y^{2}}$, which is antibonding with the nitrogen atoms, is now occupied, the $\mathrm{Fe}-\mathrm{N}$ bond elongates so that the average $\mathrm{Fe}-\mathrm{N}$ bond measures $2.16 \AA$ $(2.16 \AA)$ for $S=2$ vs $2.09 \AA(2.08 \AA)$ for the IS state (see the SI for isosurfaces of the charge density for various energy windows in the IS and $\mathrm{HS} \mathrm{FeP-Pt(111)).}$

The spin density, i.e., the difference between the electron densities of the two spin components, is shown in Figure 7 for 


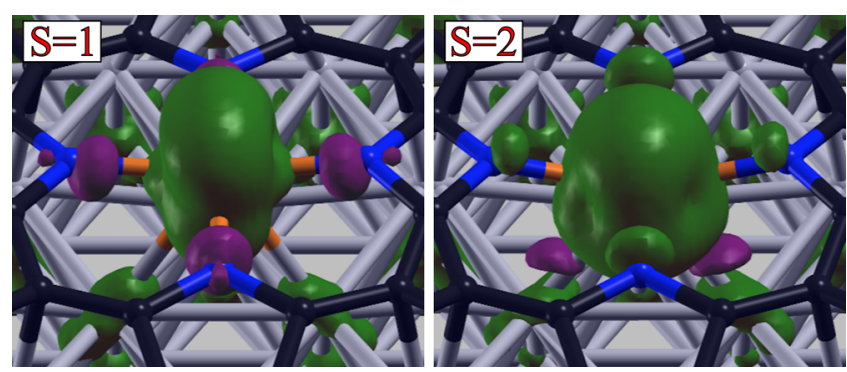

Figure 7. Isosurfaces (isovalue $= \pm 0.02 e / \AA^{3}$ ) of the spin density, i.e., the difference between the electron densities of the two spin components, for the optimized $S=1$ and $S=2 \mathrm{FeP}-\mathrm{Pt}(111)$ systems as calculated with the vdW-DF-optPBE functional at the $\mathrm{B}_{45}$ site. The purple and green colors denote the two different spin components.

the $S=1$ and $S=2$ states that were optimized on $\operatorname{Pt}(111)$. The plots show that within the molecule the unpaired electron density resides, for the most part, close to the iron atom in both systems. A little spin density is induced on the nitrogen atoms in both the IS and HS states. In both systems the iron atom is in close enough proximity to the surface so as to induce spin density within the platinum atoms in the surface.

Simulated STM. Some of the computed properties are, of course, accessible experimentally. Notably, scanning tunneling microscopy (STM) is a powerful local probe of the structure and electronic properties of single molecule adsorbates. The tunnel current between the probing tip of the STM and the sample is directly dependent on the local density of states of the molecules, ${ }^{65}$ so that STM is the method of choice to investigate local electronic properties, structural details, and the spin state in many studies. To guide experimentalists, and to illustrate the delicate interplay between structural and electronic properties plus the spin state, we simulate here STM images for the intermediate and high $\mathrm{Fe}$ spin states of $\mathrm{FeP}$ on $\mathrm{Pt}(111)$ using the vdW-DF-optPBE functional.

Shown in Figure 8 are select simulated STM images at bias voltages of $-1,-0.04,+0.04$, and $+1 \mathrm{~V}$. Notable differences between images for $S=1$ and $S=2$ are visible at the site of the central $\mathrm{Fe}$ ion, where the $\mathrm{Fe}$ in the IS state appears brighter and more localized and the $\mathrm{Fe}$ in the HS state is darker and more blurry. As has been discussed, a HS state results in a short $\mathrm{Fe}-$ Pt distance, and vice versa. As noted previously, the computed Bader charges showed that a shorter $\mathrm{Fe}-\mathrm{Pt}$ bond results in a somewhat larger molecule $\rightarrow$ surface charge transfer.

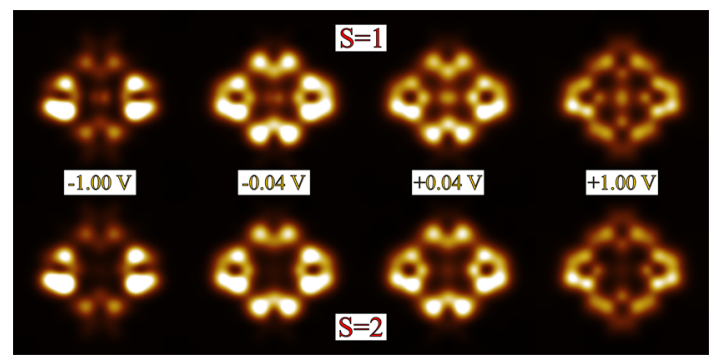

Figure 8. Simulated STM images of $\mathrm{FeP}$ on the $\mathrm{Pt}(111)$ surface using a voltage bias of \pm 1.00 and $\pm 0.04 \mathrm{~V}$, and a tip height of approximately $3.5 \AA$ from the top of the $\mathrm{Pt}(111)$ surface for the (top) intermediate and (bottom) high Fe spin states as calculated with the vdW-DFoptPBE functional. The Fe atom in the IS state is brighter than in the HS state, and the Fe atom in the HS state appears blurrier.
This effect should, in principle, be experimentally observable with STM. The simulated STM images in Figure 8 were derived by calculating the partial charge density between the respective voltages and the Fermi level and should as such be observable with simple metallic STM tips. It is noted, however, that the differences in the STM images are small and might be reduced further in experiments where tip convolution effects contribute to a reduced lateral resolution; however, the simulation illustrates a basic mechanism on how the molecular spin state can modulate the tunnel current in STM. This contrast mechanism is different from standard spin-polarized STM imaging where the tunneling of electrons between tip and sample itself is spin dependent due to magneto tunnel resistance effects based on asymmetries in the DOS for up and down spin electrons. ${ }^{66}$

\section{CONCLUSIONS}

The value of this work is in the systematic comparison of the computational results obtained on a popular experimental system. Our first-principles studies of the adsorption of the iron porphyrin $(\mathrm{FeP})$ molecule to the $\mathrm{Pt}(111)$ surface illustrate that the van der Waals (vdW) density functional employed within the calculations can significantly affect the geometries of the most stable binding sites and binding energies for this chemisorbed system. In accordance with previous research carried out on benzene adsorbed to the $\mathrm{Pt}(111)$ surface, ${ }^{39,40}$ the vdW-DF-optPBE and vdW-DF-optB88 functionals were found to yield similar results, with the binding energies computed for the former functional being somewhat smaller than those obtained with the latter. The vdW-DF-revPBE functional, however, yielded binding energies that are about half as large as the ones computed with vdW-DF-optB88 and the most stable binding site found differed substantially from the ones obtained with the other two functionals. Because of the good agreement of experimental binding energies of benzene to $\mathrm{Pt}(111)$ with those calculated via the vdW-DF-optPBE and vdW-DF-optB88 functionals, ${ }^{39,40}$ we assumed that these functionals are better suited to describe the interaction of FeP with $\mathrm{Pt}(111)$.

In the most stable binding site of $\mathrm{FeP}$ to $\mathrm{Pt}(111)$ the iron atom lies on top of a bridge (B) site, resulting in two $\mathrm{Fe}-\mathrm{Pt}$ bonds. The $\mathrm{B}_{45}$ site is more stable than the $\mathrm{B}_{0}$ because this geometry allows for a greater number of $\mathrm{C}-\mathrm{Pt}$ bonds to form. In general, the strength of the binding energy was found to correlate with the number of $\mathrm{Fe}-\mathrm{Pt}$ and $\mathrm{C}-\mathrm{Pt}$ bonds in the chemisorbed system, with the former interaction being more important than the latter for the vdW-DF-optPBE functional.

Adsorption led to a switching of the spin of iron from the intermediate, $S=1$, state with the $\left(\mathrm{d}_{x y}\right)^{2}\left(\mathrm{~d}_{\pi}\right)^{3}\left(\mathrm{~d}_{z}^{2}\right)^{1}$ occupation that is preferred in the gas phase, to the high, $S=2$, state with the $\left(d_{x y}\right)^{2}\left(d_{\pi}\right)^{2}\left(d_{z}^{2}\right)^{1}\left(d_{x^{2}-y^{2}}\right)^{1}$ occupation on $\operatorname{Pt}(111)$. The spin switch occurs because of the elongation of the $\mathrm{Fe}-\mathrm{N}$ bond that is caused by the puckering of the iron atom below the plane of the porphyrin, which occurs so that it can bind to the platinum atoms. A Bader analysis revealed that molecule $\rightarrow$ surface charge transfer occurs upon adsorption. The $S=1$ configuration adsorbed to the $\mathrm{B}_{45}$ site was found to be less stable than the $S=2$ state because it had a larger $\mathrm{Fe}-\mathrm{Pt}$ distance. At all of the binding sites considered, the high spin state was preferred over the intermediate spin state. We demonstrated the correlation between the spin state, the bond length, and the charge density at the molecule-substrate 
interface, which is experimentally accessible, for instance, with STM.

The demonstrated interplay between the structural properties of molecular adsorbates, energy level alignment, charge transfer, and concomitantly the molecular spin state is of fundamental importance, and specifically the impact of the substrate on the adsorbate-molecule properties. Thus, the presented investigation can aid in the theory-driven discovery of useful molecular magnetic structures and assist in developing strategies to design their magnetic properties for applications.

\section{ASSOCIATED CONTENT}

\section{S Supporting Information}

The Supporting Information is available free of charge on the ACS Publications website at DOI: 10.1021/acs.jpcc.6b09408.

Illustrations of the optimized geometries obtained with the vdW-DF-revPBE functional, further contour and isosurface plots of the CDD, Bader charges, charge densities for select energy regions of the IS and HS FeP$\mathrm{Pt}(111)$ systems, PDOS of FeP in the gas phase and the $S=2$ spin state coupled with isosurfaces of the partial charge densities for select energy regions with the vdWDF-optPBE functional, diagram illustrating the symmetry elements in the FeP-Pt(111) system, analysis of $\mathrm{FeP}$ and $\mathrm{FeP}$ coordinated to imidazole, relative energies of different spin states for various functionals and choices of $U_{\text {eff }}$, calculated workfunctions of the FeP-Pt(111) systems, and coordinates of the optimized geometries (PDF)

\section{AUTHOR INFORMATION}

\section{Corresponding Authors}

*E-mail: jab95@psu.edu.

*E-mail: ezurek@buffalo.edu.

\section{ORCID}

Daniel P. Miller: 0000-0003-1507-2667

\section{Notes}

The authors declare no competing financial interest.

\section{ACKNOWLEDGMENTS}

J.B. thanks the National Science Foundation (CHE-1305660) for financial support. Support from the Center of Computational Research at the University at Buffalo is acknowledged. E.Z. thanks the Alfred P. Sloan Foundation for a Research Fellowship (2013-2015). The authors would like to thank Susanne Simkovitch for her preliminary investigations of the $\mathrm{FeP}$ molecule in the gas phase.

\section{REFERENCES}

(1) Enders, A.; Skomski, R.; Honolka, J. Magnetic surface nanostructures. J. Phys.: Condens. Matter 2010, 22, 433001.

(2) Christou, G.; Gatteschi, D.; Hendrickson, D. N.; Sessoli, R. Single-molecule magnets. MRS Bull. 2000, 25, 66-71.

(3) Gutlich, P.; Garcia, Y.; Goodwin, H. A. Spin crossover phenomena in Fe(II) complexes. Chem. Soc. Rev. 2000, 29, 419-427.

(4) Kurmoo, M. Magnetic metal-organic frameworks. Chem. Soc. Rev. 2009, 38, 1353-1379.

(5) Barth, J. V.; Costantini, G.; Kern, K. Engineering atomic and molecular nanostructures at surfaces. Nature 2005, 437, 671-679.

(6) Rojas, G.; et al. Self-assembly and properties of nonmetalated tetraphenyl-porphyrin on metal substrates. J. Phys. Chem. C 2010, 114, 9408-9415.
(7) Bhandary, S.; et al. Manipulation of spin state of iron porphyrin by chemisorption on magnetic substrates. Phys. Rev. B: Condens. Matter Mater. Phys. 2013, 88, 024401.

(8) Heinrich, B. W.; Braun, L.; Pascual, J. I.; Franke, K. J. Tuning the magnetic anisotropy of single molecules. Nano Lett. 2015, 15, 40244028.

(9) Wang, W.; Pang, R.; Kuang, G.; Shi, X.; Shang, X.; Liu, P. N.; Lin, $\mathrm{N}$. Intramolecularly resolved Kondo resonance of high-spin $\mathrm{Fe}(\mathrm{II})$ porphyrin adsorbed on $\mathrm{Au}(111)$. Phys. Rev. B: Condens. Matter Mater. Phys. 2015, 91, 045440.

(10) Auwärter, W.; Écija, D.; Klappenberger, F.; Barth, J. V. Porphyrins at interfaces. Nat. Chem. 2015, 7, 105-120.

(11) Wende, H.; et al. Substrate-induced magnetic ordering and switching of iron porphyrin molecules. Nat. Mater. 2007, 6, 516-520.

(12) Zhang, Q.; Kuang, G.; Pang, R.; Shi, X.; Lin, N. Switching molecular Kondo effect via supramolecular interaction. ACS Nano 2015, 9, 12521-12528.

(13) Bennett, J. A.; Sterling, K. L.; Pander, J. E. Direct metal substitution of electropolymerized ferriprotoporphyrin: A simple electrode-modification process for developing electrocatalytic materials. ECS Electrochem. Lett. 2013, 2, H37-H39.

(14) Bennett, J. A.; Neiswonger, M. A.; Wheeler, C. D.; Pander, J. E.; McKinney, S. E. Cyanide-coordinated Fe(III) meso-tetra(4-carboxyphenyl) porphyrin as a possible electrocatalytic material for selective $\mathrm{H}_{2} \mathrm{~S}$ oxidation. J. Electrochem. Soc. 2012, 159, F119-F124.

(15) Bennett, J. A.; Wheeler, C. D.; Sterling, K. L.; Chiodo, A. M. Exploring dicyano-ferriprotoporphyrin as a novel electrocatalytic material for selective $\mathrm{H}_{2} \mathrm{~S}$ gasotransmitter detection. Electrochim. Acta 2013, 88, 86-93.

(16) Leung, K.; Rempe, S. B.; Schultz, P. A.; Sproviero, E. M.; Batista, V. S.; Chandross, M. E.; Medforth, C. J. Density functional theory and $\mathrm{DFT}+\mathrm{U}$ study of transition metal porphines adsorbed on $\mathrm{Au}(111)$ surfaces and effects of applied electric fields. J. Am. Chem. Soc. 2006, $128,3659-3668$.

(17) Brena, B.; Herper, H. C. Influence of ligands on the electronic and magnetic properties of $\mathrm{Fe}$ porphyrin in gas phase and on $\mathrm{Cu}(001)$. J. Appl. Phys. 2015, 117, 17B318.

(18) Muller, M.; Diller, K.; Maurer, R. J.; Reuter, K. Interfacial charge rearrangement and intermolecular interactions: Density-functional theory study of free-base porphine adsorbed on $\mathrm{Ag}(111)$ and $\mathrm{Cu}(111)$. J. Chem. Phys. 2016, 144, 024701.

(19) Luder, J.; Sanyal, B.; Eriksson, O.; Puglia, C.; Brena, B. Comparison of van der Waals corrected and sparse-matter density functionals for the metal-free phthalocyanine/gold interface. Phys. Rev. B: Condens. Matter Mater. Phys. 2014, 89, 045416.

(20) Gottfried, J. M. Surface chemistry of porphyrins and phthalocyanines. Surf. Sci. Rep. 2015, 70, 259-379.

(21) Antczak, G.; Kamiński, W.; Sabik, A.; Zaum, C.; Morgenstern, $\mathrm{K}$. Complex surface diffusion mechanisms of cobalt phthalocyanine molecules on $\mathrm{Ag}(100)$. J. Am. Chem. Soc. 2015, 137, 14920-14929.

(22) Buchner, F.; Warnick, K.-G.; Wölfle, T.; Görling, A.; Steinrück, H.-P.; Hieringer, W.; Marbach, H. Chemical fingerprints of large organic molecules in scanning tunneling microscopy: Imaging adsorbate-substrate coupling of metalloporphyrins. J. Phys. Chem. C 2009, 113, 16450-16457.

(23) Seufert, K.; Auwärter, W.; Barth, J. V. Discriminative response of surface-confined metalloporphyrin molecules to carbon and nitrogen monoxide. J. Am. Chem. Soc. 2010, 132, 18141-18146.

(24) Lukasczyk, T.; Flechtner, K.; Merte, L. R.; Jux, N.; Maier, F.; Gottfried, J. M.; Steinrück, H.-P. Interaction of cobalt(II) tetraarylporphyrins with a $\operatorname{Ag}(111)$ surface studied with photoelectron spectroscopy. J. Phys. Chem. C 2007, 111, 3090-3098.

(25) Auwärter, W.; et al. Site-specific electronic and geometric interface structure of Co-tetraphenyl-porphyrin layers on $\mathrm{Ag}(111)$ Phys. Rev. B: Condens. Matter Mater. Phys. 2010, 81, 245403.

(26) Iancu, V.; Deshpande, A.; Hla, S. W. Manipulating Kondo temperature via single molecule switching. Nano Lett. 2006, 6, 820823. 
(27) Wu, F.; Liu, J.; Mishra, P.; Komeda, T.; Mack, J.; Chang, Y.; Kobayashi, N.; Shen, Z. Modulation of the molecular spintronic properties of adsorbed copper corroles. Nat. Commun. 2015, 6, 7547.

(28) Bhandary, S.; Ghosh, S.; Herper, H.; Wende, H.; Eriksson, O.; Sanyal, B. Graphene as a reversible spin manipulator of molecular magnets. Phys. Rev. Lett. 2011, 107, 257202.

(29) Collman, J.; Hoard, J.; Kim, N.; Lang, G.; Reed, C. Synthesis, stereochemistry, and structure-related properties of $\alpha, \beta, \gamma, \delta$ tetraphenylporphinatoiron(II). J. Am. Chem. Soc. 1975, 97, 26762681.

(30) Lang, G.; Spartalian, K.; Reed, C. A.; Collman, J. P. Mössbauer effect study of the magnetic properties of $S=1$ ferrous tetraphenylporphyrin. J. Chem. Phys. 1978, 69, 5424-5427.

(31) Hu, C.; Roth, A.; Ellison, M.; An, J.; Ellis, C.; Schulz, C.; Scheidt, W. Electronic configuration assignment and the importance of low-lying excited states in high-spin imidazole-ligated iron(II) porphyrinates. J. Am. Chem. Soc. 2005, 127, 5675-5688.

(32) Boyd, P. D. W.; Buckingham, D. A.; McMeeking, R. F.; Mitra, S. Paramagnetic anisotropy, average magnetic susceptibility, and electronic structure of intermediate-spin $S=1(5,10,15,20$ tetraphenylporphyrin)iron(II). Inorg. Chem. 1979, 18, 3585-3591.

(33) Goff, H.; La Mar, G.; Reed, C. Nuclear magnetic resonance investigation of magnetic and electronic properties of "intermediate spin" ferrous porphyrin complexes. J. Am. Chem. Soc. 1977, 99, 36413646.

(34) Mispelter, J.; Momenteau, M.; Lhoste, J. M. Proton magnetic resonance characterization of the intermediate $(S=1)$ spin state of ferrous porphyrins. J. Chem. Phys. 1980, 72, 1003-1012.

(35) Kitagawa, T.; Teraoka, J. The resonance Raman spectra of intermediate-spin ferrous porphyrin. Chem. Phys. Lett. 1979, 63, 443446.

(36) Radoń, M.; Pierloot, K. Binding of $\mathrm{CO}, \mathrm{NO}$, and $\mathrm{O}_{2}$ to Heme by Density functional and multireference ab initio calculations. J. Phys. Chem. A 2008, 112, 11824-11832.

(37) Rovira, C.; Kunc, K.; Hutter, J.; Ballone, P.; Parrinello, M. Equilibrium geometries and electronic structure of iron-porphyrin complexes: A density functional study. J. Phys. Chem. A 1997, 101, 8914-8925.

(38) Radoń, M. Spin-state energetics of heme-related models from DFT and coupled cluster calculations. J. Chem. Theory Comput. 2014, 10, 2306-2321.

(39) Carrasco, J.; Liu, W.; Michaelides, A.; Tkatchenko, A. Insight into the description of van der Waals forces for benzene adsorption on transition metal (111) surfaces. J. Chem. Phys. 2014, 140, 084704.

(40) Yildirim, H.; Greber, T.; Kara, A. Trends in adsorption characteristics of benzene on transition metal surfaces: Role of surface chemistry and van der Waals interactions. J. Phys. Chem. C 2013, 117, 20572-20583.

(41) Liu, W.; Carrasco, J.; Santra, B.; Michaelides, A.; Scheffler, M.; Tkatchenko, A. Benzene adsorbed on metals: Concerted effect of covalency and van der Waals bonding. Phys. Rev. B: Condens. Matter Mater. Phys. 2012, 86, 245405.

(42) Gautier, S.; Steinmann, S. N.; Michel, C.; Fleurat-Lessard, P.; Sautet, P. Molecular adsorption at $\mathrm{Pt}(111)$. How accurate are DFT functionals? Phys. Chem. Chem. Phys. 2015, 17, 28921-28930.

(43) Klimeš, J.; Bowler, D. R.; Michaelides, A. Chemical accuracy for the van der Waals density functional. J. Phys.: Condens. Matter 2010, 132, 022201.

(44) Román-Pérez, G.; Soler, J. M. Efficient implementation of a van der Waals density functional: Application to double-wall carbon nanotubes. Phys. Rev. Lett. 2009, 103, 096102.

(45) Klimeš, J.; Bowler, D. R.; Michaelides, A. Van der Waals density functionals applied to solids. Phys. Rev. B: Condens. Matter Mater. Phys. 2011, 83, 195131.

(46) Klimeš, J.; Michaelides, A. Perspective: Advances and challenges in treating van der Waals dispersion forces in density functional theory. J. Chem. Phys. 2012, 137, 120901.

(47) Gambardella, P.; Rusponi, S.; Veronese, M.; Dhesi, S. S.; Grazioli, C.; Dallmeyer, A.; Cabria, I.; Zeller, R.; Dederichs, P. H.;
Kern, K.; Carbone, C.; Brune, H. Giant magnetic anisotropy of single cobalt atoms and nanoparticles. Science 2003, 300, 1130-1133.

(48) Komelj, M.; Steiauf, D.; Fähnle, M. Influence of the substrate on the magnetic anisotropy of monatomic nanowires. Phys. Rev. B: Condens. Matter Mater. Phys. 2006, 73, 134428.

(49) Bornemann, S.; Minár, J.; Staunton, J. B.; Honolka, J.; Enders, A.; Kern, K.; Ebert, H. Magnetic anisotropy of deposited transition metal clusters. Eur. Phys. J. D 2007, 45, 529-534.

(50) Skomski, R.; Zhang, J.; Sessi, V.; Honolka, J.; Kern, K.; Enders, A. Substrate-controlled growth and magnetism of nanosize Fe clusters on Pt. J. Appl. Phys. 2008, 103, 07D519.

(51) Kresse, G.; Furthmuller, J. Efficient iterative schemes for ab initio total-energy calculations using a plane-wave basis set. Phys. Rev. B: Condens. Matter Mater. Phys. 1996, 54, 11169-11186.

(52) Dion, M.; Rydberg, H.; Schröder, E.; Langreth, D. C.; Lundquist, B. I. Van der Waals density functional for general geometries. Phys. Rev. Lett. 2004, 92, 246401.

(53) Kresse, G.; Joubert, D. From ultrasoft pseudopotentials to the projector augmented-wave method. Phys. Rev. B: Condens. Matter Mater. Phys. 1999, 59, 1758-1775.

(54) Monkhorst, H. J.; Pack, J. D. Special points for brillouin-zone integrations. Phys. Rev. B 1976, 13, 5188-5192.

(55) Dudarev, S. L.; Botton, G. A.; Savrasov, S. Y.; Humphreys, C. J.; Sutton, A. P. Electron-energy-loss spectra and the structural stability of nickel oxide: An LSDA+U study. Phys. Rev. B: Condens. Matter Mater. Phys. 1998, 57, 1505-1509.

(56) Panchmatia, P. M.; Sanyal, B.; Oppeneer, B. M. GGA+U modeling of structural, electronic, and magnetic properties of iron porphyrin-type molecules. Chem. Phys. 2008, 343, 47-60.

(57) Ali, M. E.; Sanyal, B.; Oppeneer, P. M. Electronic structure, spin-states, and spin-crossover reaction of heme-related Fe-porphyrins A theoretical perspective. J. Phys. Chem. B 2012, 116, 5849-5898.

(58) Hanwell, M. D.; Curtis, D. E.; Lonie, D.; Vandermeersch, T.; Zurek, E.; Hutchison, G. R. Avogadro: An advanced semantic chemical editor, visualization, and analysis platform. J. Cheminf. 2012, 4, 1-17. See http://avogadro.openmolecules.net/.

(59) Kokalj, A. Computer graphics and graphical user interfaces as tools in simulations of matter at the atomic scale. Comput. Mater. Sci. 2003, 28, 155-168.

(60) te Velde, G.; Bickelhaupt, F. M.; Baerends, E. J.; Fonseca Guerra, C.; van Gisbergen, S. J. A.; Snijders, J. G.; Ziegler, T. Chemistry with ADF. J. Comput. Chem. 2001, 22, 931-967.

(61) Baerends, E. J.; et al. ADF2013.01; http://www.scm.com.

(62) Perdew, J. P.; Burke, K.; Ernzerhof, M. Generalized gradient approximation made simple. Phys. Rev. Lett. 1996, 77, 3865-3868.

(63) Murphy, C. J.; et al. Effect of BN/CC isosterism on the thermodynamics of surface and bulk binding: 1,2-Dihydro-1,2azaborine vs benzene. J. Phys. Chem. C 2015, 119, 14624-14631.

(64) Hieringer, W.; Flechtner, K.; Kretschmann, A.; Seufert, K.; Auwärter, W.; Barth, J. V.; Görling, A.; Steinrück, H.-P.; Gottfried, J. $M$. The surface trans effect: Influence of axial ligands on the surface chemical bonds of adsorbed metalloporphyrins. J. Am. Chem. Soc. 2011, 133, 6206-6222.

(65) Tersoff, J.; Hamann, D. R. Theory of the scanning tunneling microscope. Phys. Rev. B: Condens. Matter Mater. Phys. 1985, 31, 805813.

(66) Bode, M.; Getzlaff, M.; Wiesendanger, R. Spin-polarized vacuum tunneling into the exchange-split surface state of $\mathrm{Gd}(0001)$. Phys. Rev. Lett. 1998, 81, 4256-4259. 


\section{Supporting Information: Electronic Structure of Iron Porphyrin Adsorbed to the $\operatorname{Pt}(111)$ Surface}

Section S1: Preferred Binding Site and Geometric Info Calculated Using vdW-DF-revPBE.

Section S2: Split-up CDD of the $S=1$ and $S=2$ FeP-Pt(111) Systems......

Section S3: Contour plots of the CDD of the $S=1$ and $S=2$ FeP-Pt(111) Systems. S5-6

Section S4: Bader Charges of FeP in the Gas Phase and on $\mathrm{Pt}(111) \ldots$ .$S 7$

Section S5: PDOS of the $\mathrm{S}=2 \mathrm{FeP}$ in the Gas Phase with Partial Charge Densities. S8

Section S6: Symmetry in the FeP-Pt(111) System.

Section S7: Four Coordinate FeP Versus Five Coordinate FeP. S10-12

Section S8: Workfunction of FeP-Pt(111) $\mathrm{S} 13$

Section S9: Partial Charge Densities of the $S=1$ and $S=2$ FeP-Pt(111) Systems.

Section S10: Coordinates of Isolated FeP and Pt(111) Surfaces (POSCAR format)........ S28-38

Section S11: Coordinates of FeP on the Pt(111) Surface (POSCAR format). S39-117 
Section S1: Preferred Binding Site and Geometric Info Calculated Using vdW-DF-revPBE

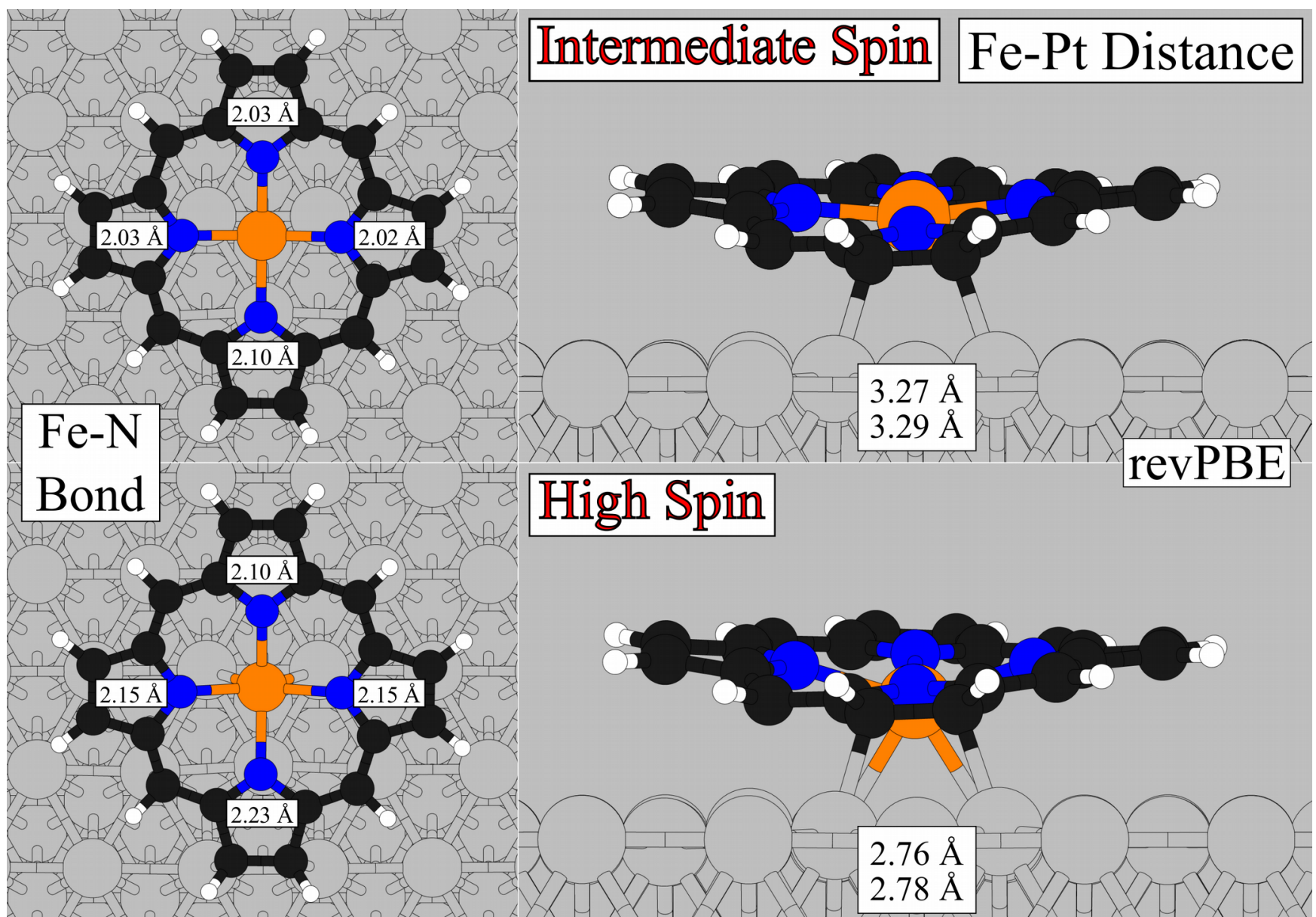

Figure S1: The preferred binding site $\left(\mathrm{H}_{\mathrm{hp}}-45\right)$ and geometric info calculated for the $S=1$ (intermediate spin) and $S=2$ (high spin) FeP-Pt(111) systems using the vdW-DF-revPBE functional. The Fe-N bond distances are shown to the left while the Fe-Pt bond distances are shown to the right. 
Section S2: Split-up CDD of the $S=1$ and $S=2$ FeP-Pt(111) Systems

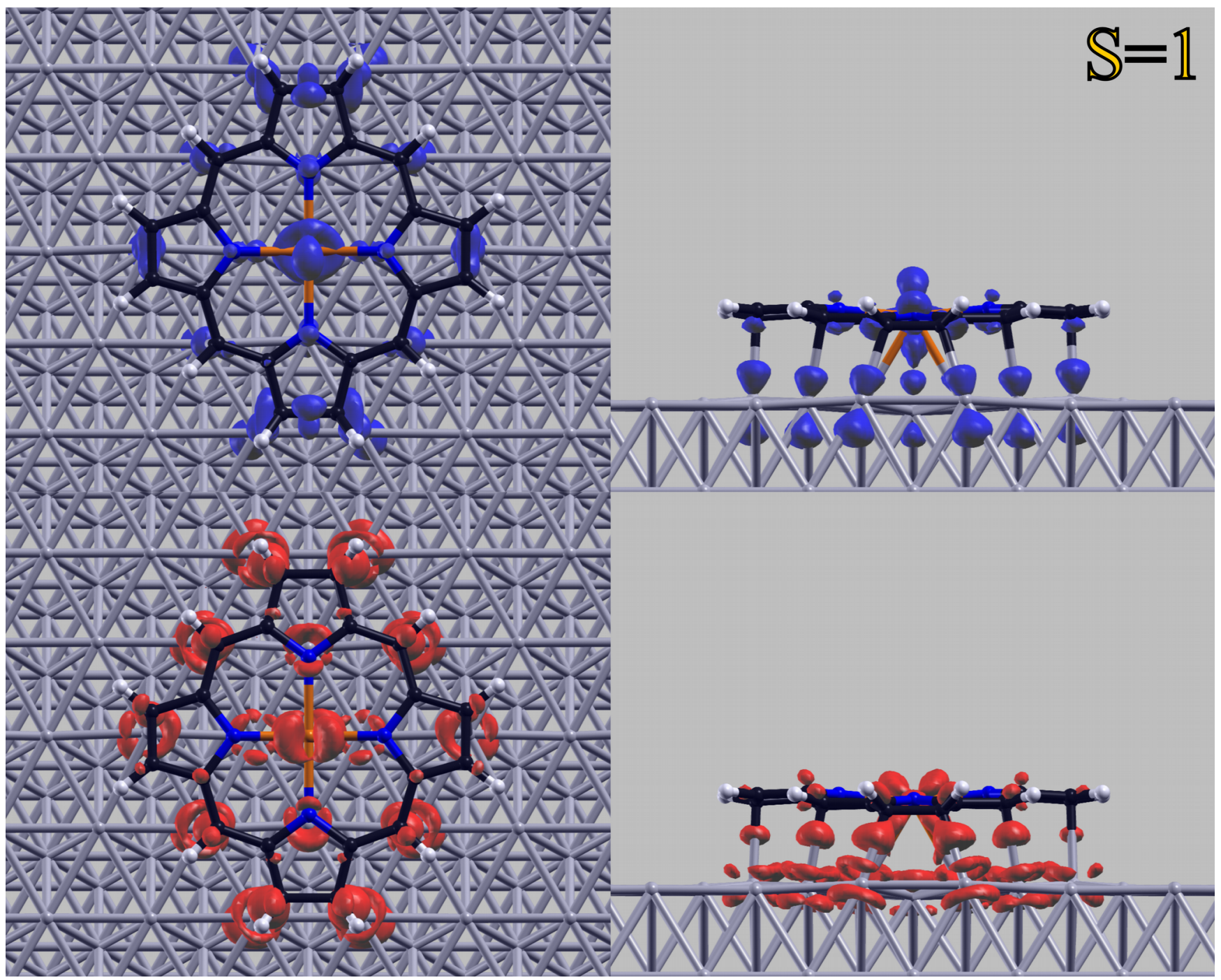

Figure S2: The split-up charge density difference (CDD) of the $S=1$ FeP-Pt(111) system as calculated with vdW-DF-optPBE. The red/blue represent a gain/loss of electronic charge density (isovalue $=+/-0.04 \mathrm{e} / \AA^{3}$ ). 

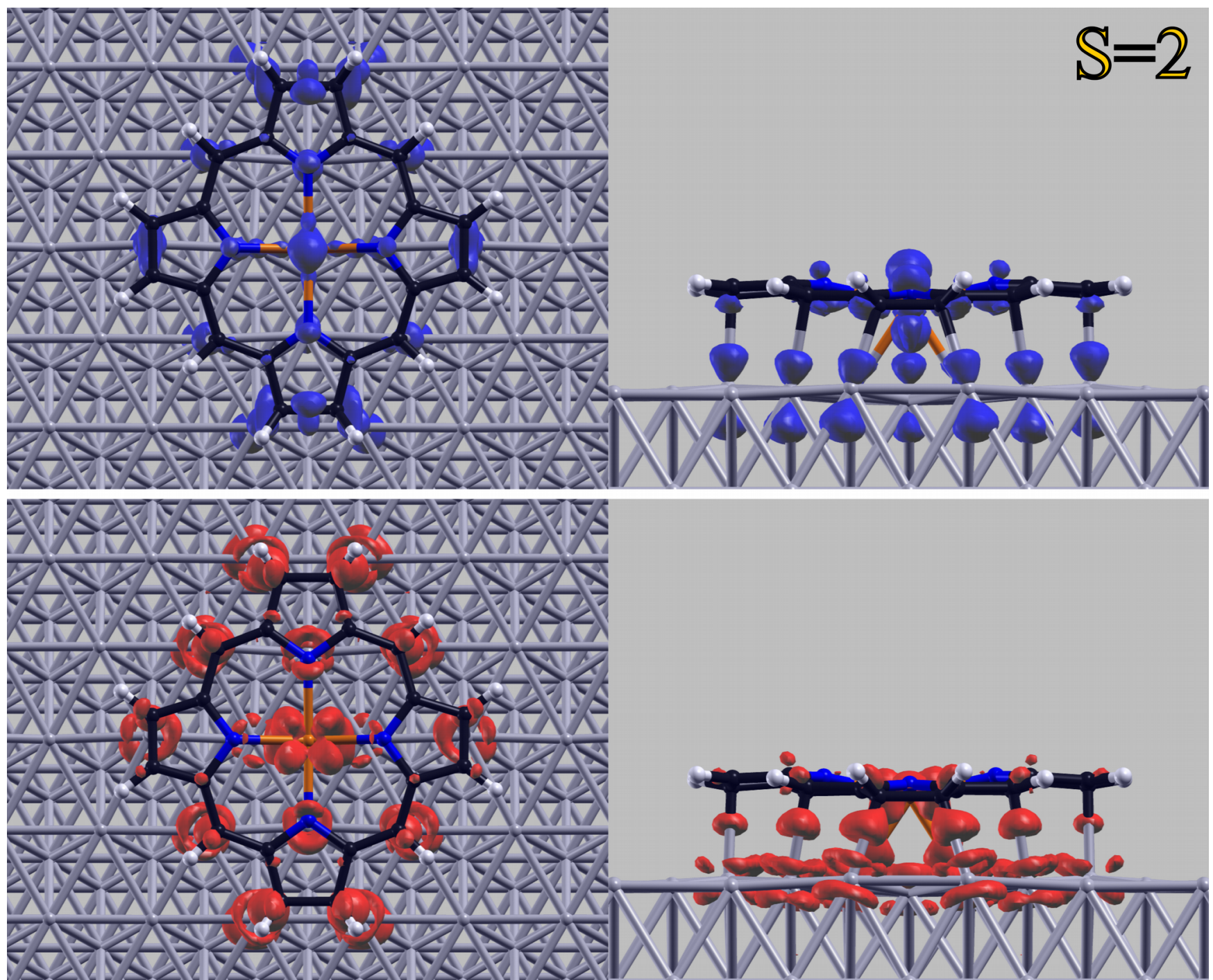

Figure S3: The split-up charge density difference (CDD) of the $S=2$ FeP-Pt(111) system as calculated with vdW-DF-optPBE. The red/blue represent a gain/loss of electronic charge density (isovalue $=+/-0.04 \mathrm{e} / \AA^{3}$ ). 
Section S3: Contour plots of the CDD of the $S=1$ and $S=2$ FeP-Pt(111) Systems<smiles>[SiH2]I</smiles>
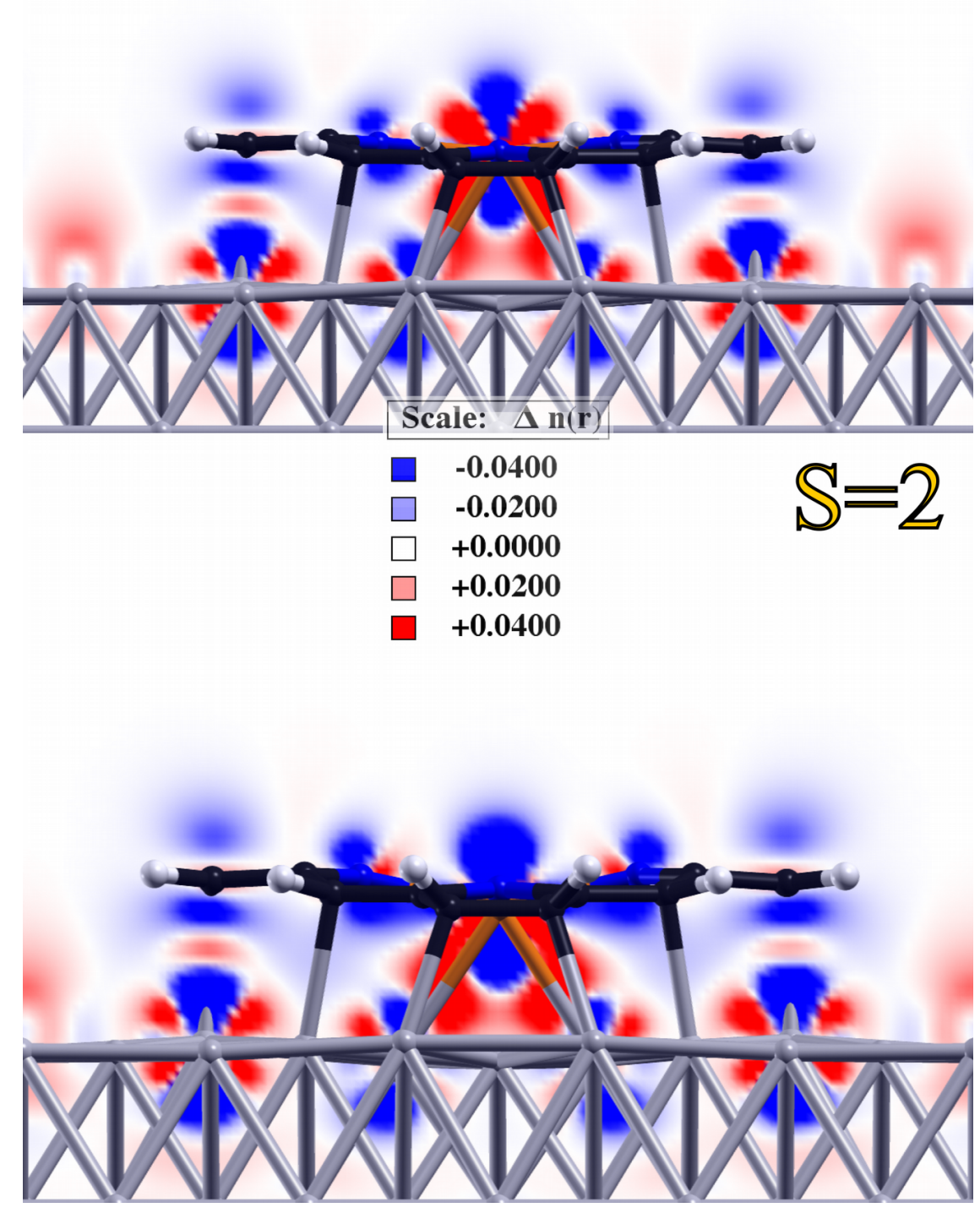

Figure S4: Contour plot of the CDD bisecting the middle of the FeP molecule (at the Fe atom) adsorbed to $P t(111)$ in the $S=1$ and $S=2$ spin state as calculated with vdW-DF-optPBE. Note the large amount of charge transfer between FeP and $\mathrm{Pt}(111)$ in both spin states. The units are e/ $\AA^{3}$ in the scale bar with red/blue representing charge gain/loss. 

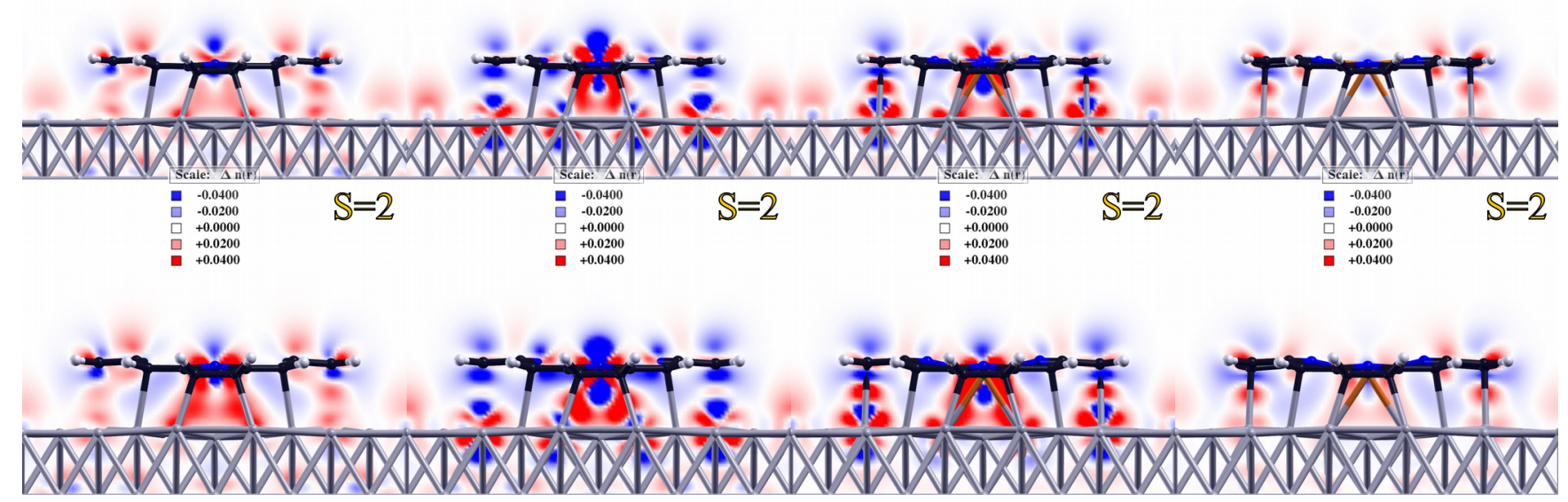

Figure S5: Contour plot of the CDD of the FeP molecule adsorbed to $\mathrm{Pt}(111)$ in the $S=1$ and $S=2$ spin states as calculated with vdW-DF-optPBE. The plane for which the CDD is shown cuts through different portions of the Fe-N bond with the leftmost plane being closest to the viewer, the rightmost image being the farthest from the viewer, and the $2^{\text {nd }}$ and $3^{\text {rd }}$ images being just below and just above the Fe atom. Note the slightly larger amount of Pauli repulsion within the $S=1$ spin state versus the $S=2$ spin state within each contour plot of the CDD. The units are e/ $\AA^{3}$ in the scale bar with red/blue representing charge gain/loss. 

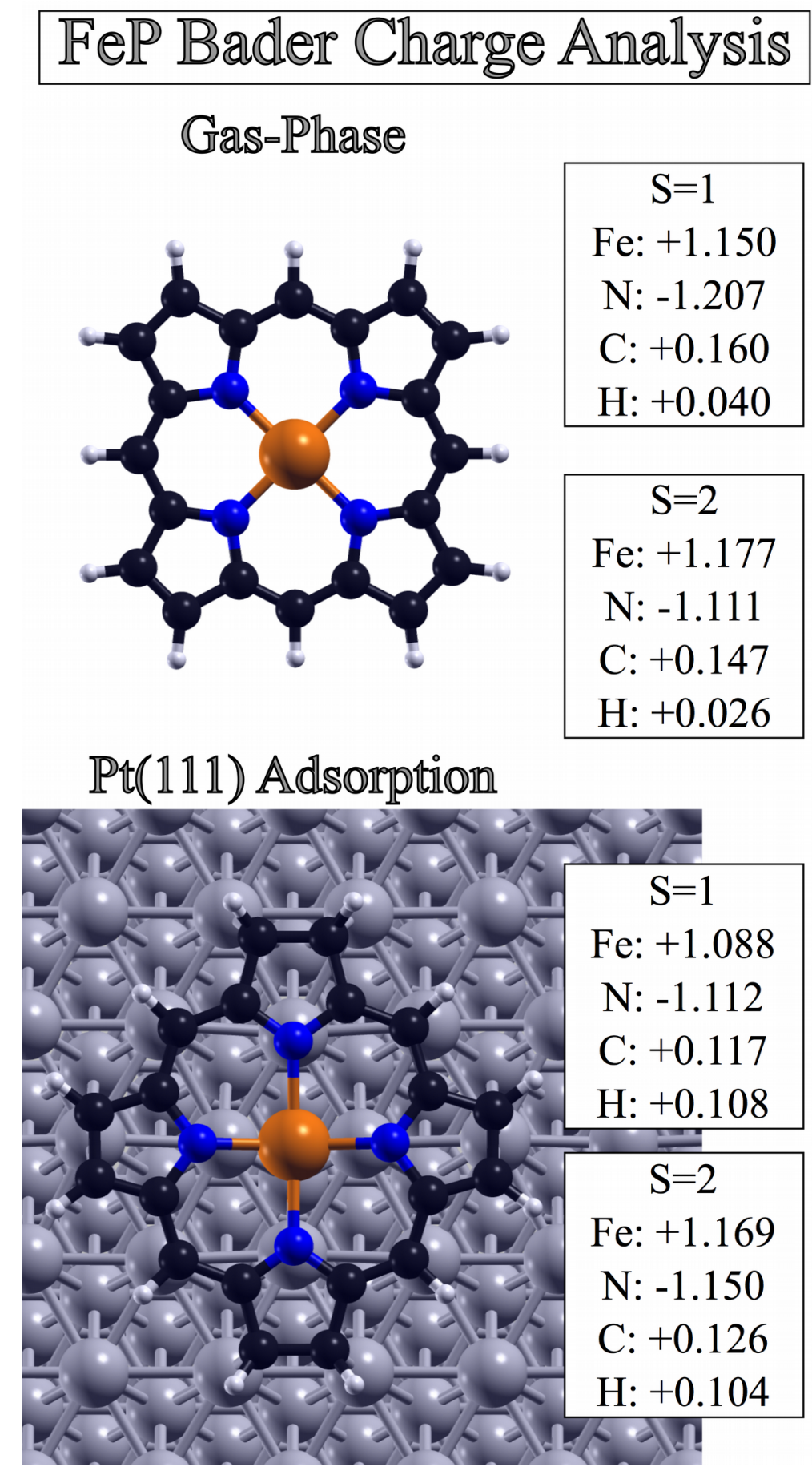

Figure S6: The averaged Bader charges (units of $e$ ) of the individual atom types in FeP within the gas phase $(S=1)$ and upon the $\operatorname{Pt}(111)$ surface in the $S=1$ and $S=2$ spin states. The hydrogen, carbon, nitrogen, iron, and platinum atoms are colored white, black, blue, orange, and gray, respectively. Calculated with vdW-DF-optPBE. A + sign represents a positive charge (loss of electrons) while a - sign represents a negative charge (gain of electrons). 
Section S5: PDOS of the $\mathrm{S}=2 \mathrm{FeP}$ in the Gas Phase with Partial Charge Densities
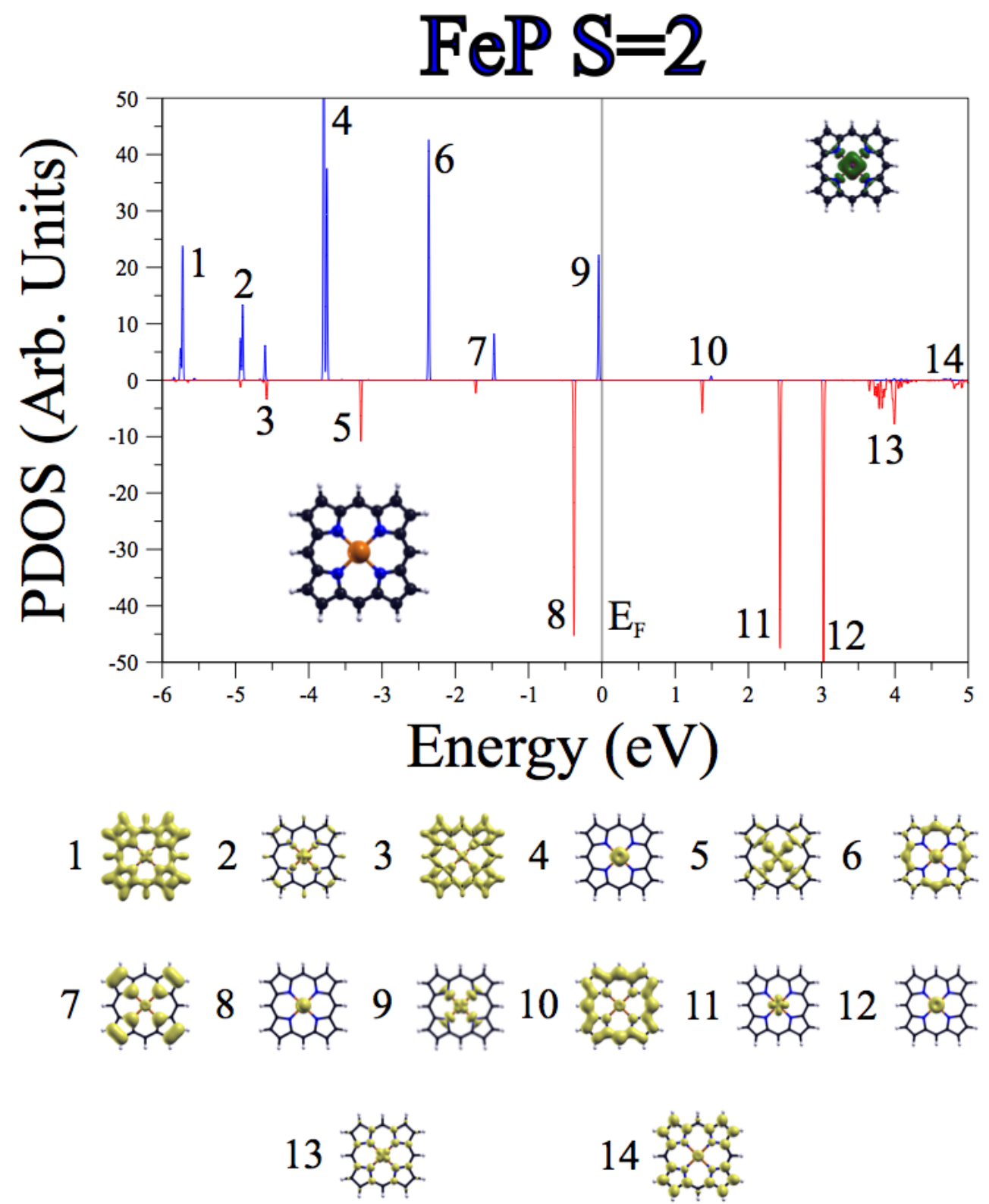

Figure S7: The projected densities of states (PDOS) for the Fe d-states in the isolated S=2 FeP molecule, coupled with isosurfaces of partial charge densities for the various peaks (isovalues = 0.01-0.1 $\mathrm{e} / \AA^{3}$ ) calculated with the vdW-DF-optPBE functional. An isosurface of the spin density (isovalue $=+/-0.01 \mathrm{e} / \AA^{3}$ ) is given in the inset. 
Section S6: Symmetry in the FeP-Pt(111) System

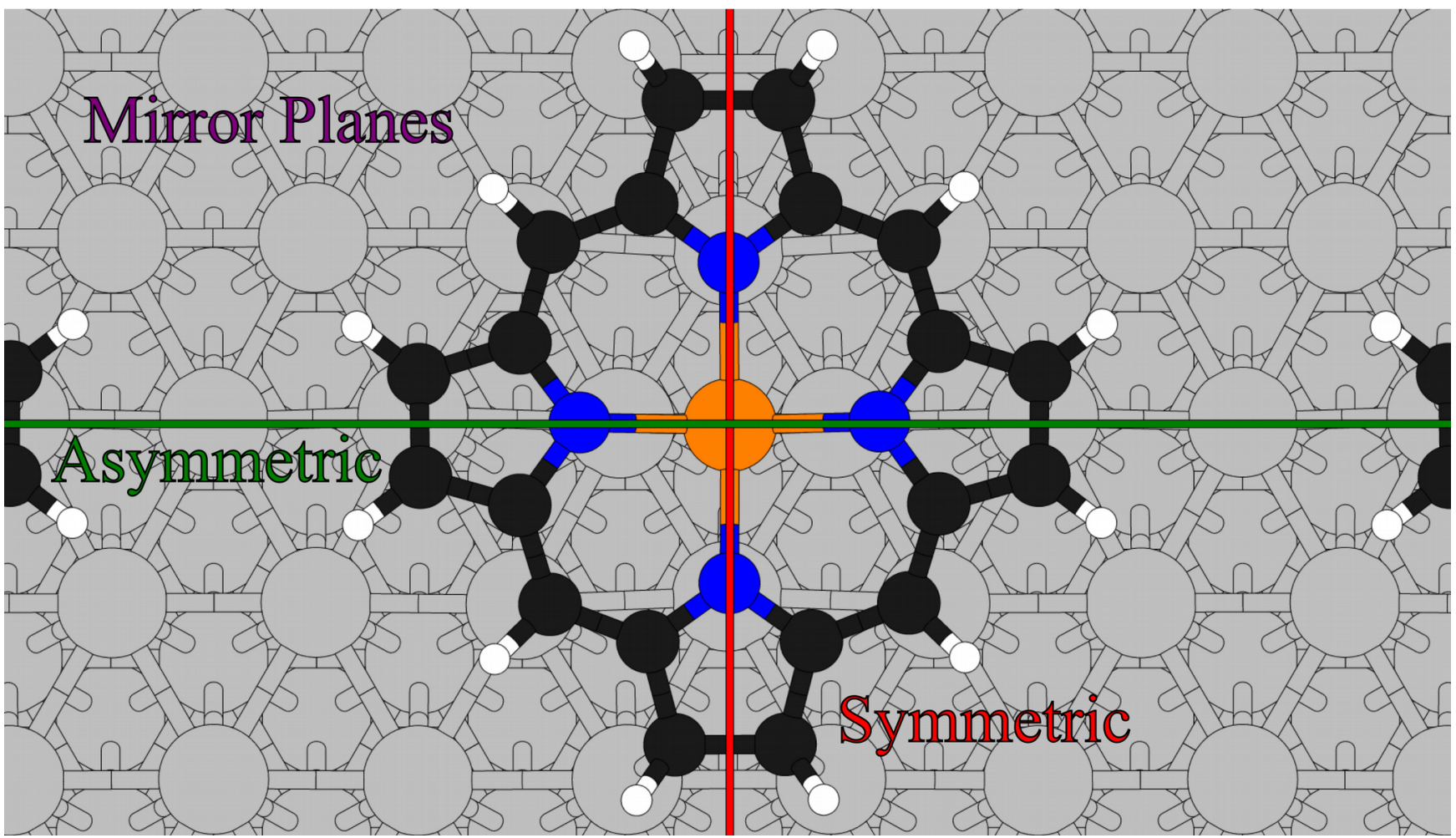

Figure S8: FeP on Pt(111) designating the mirror plane in FeP that is retained upon adsorption to the B-45 site (red). The remaining mirror planes of FeP are lost upon adsorption, with one of the three being depicted (green). 
Section S7: Four Coordinate FeP Versus Five Coordinate FeP

4 coordinate FeP<smiles>N[R](N)(N)N</smiles>

$S=2$

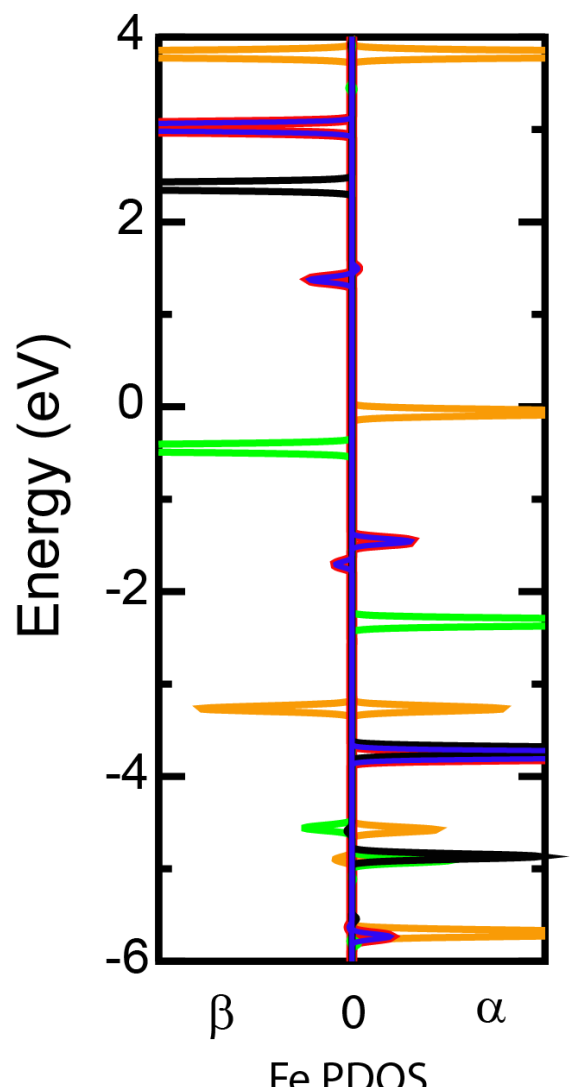

5 coordinate FeP

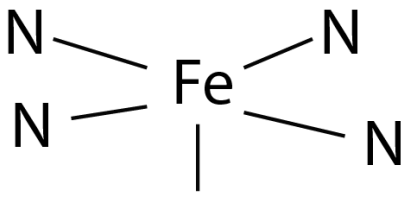

$\mathrm{N}$

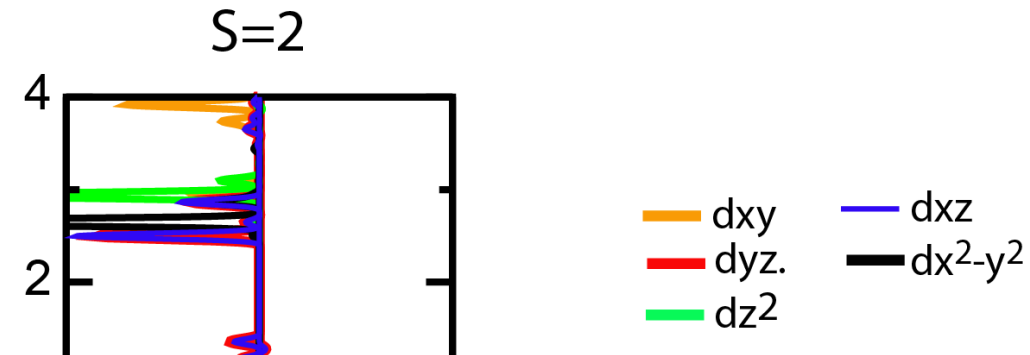

Figure S9: (Left) Projected density of states of four coordinate FeP for the Fe d states in the $S=2$ state. (Right) Projected density of states of five coordinate FeP+imidazole for the Fe d states in the $S=2$ state. The main differences between the two DOS plots is the position of the states arising from the $\mathrm{dz}^{2}$ orbitals. This is also observed in the FeP-Pt(111) DOS. 


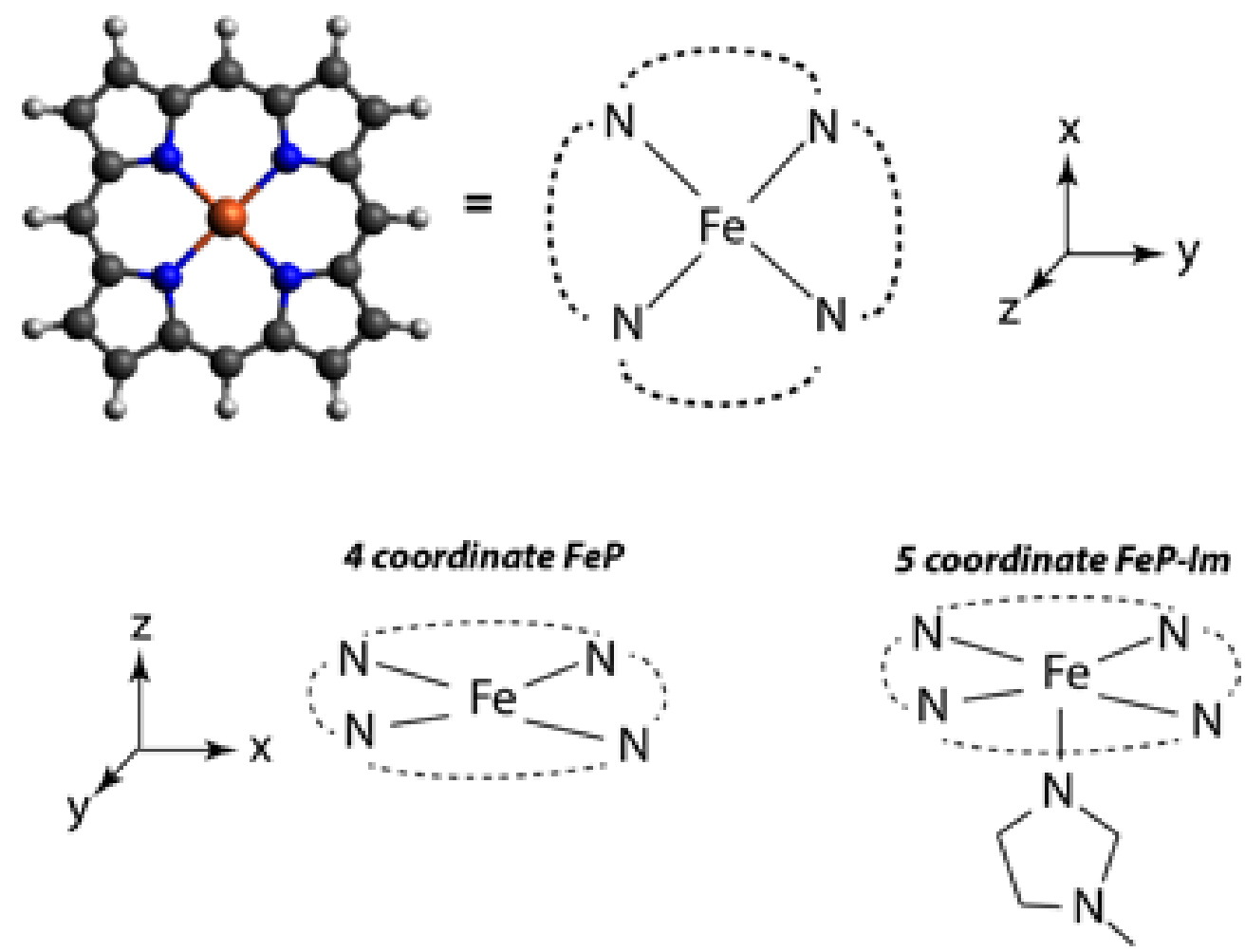

Figure S10: (Top) A sketch of the FeP model, which has a four-coordinate Fe center. (Bottom) FeP is shown alongside the FeP-Im model; FeP-Im is formed when 1-methylimidazole (Im) is bound to FeP to create a five-coordinate Fe center. The Im ligand was modeled such that the plane of the imidazole ring lies directly over $\mathrm{N}-\mathrm{Fe}-\mathrm{N}$ bonds that are in the FeP fragment below it.

In Table $S 1$, we show the relative energies between the $S=1$ and $S=2$ states, $\Delta E_{S}$, that were obtained from DFT computations on the FeP and FeP-Im models which are illustrated in Figure S10. The vdW$\mathrm{DF}$-optPBE results, in Table $\mathrm{S} 1$, are shown alongside $\Delta \mathrm{E}_{\mathrm{S}}$ values that were obtained with the PBE and $\mathrm{PBE}+\mathrm{D} 3$ functionals; $\mathrm{PBE}+\mathrm{D} 3$ refers to the $\mathrm{PBE}$ functional being used in tandem with the post-SCF dispersion corrections that were proposed by Grimme. The tendency of PBE to overstabilize the $S=1$ state and the stabilization of the $\mathrm{S}=2$ state as $\mathrm{U}-\mathrm{J}$ is increased are well-known, ${ }^{\mathrm{S} 1}$ and we reproduce those results here. The vdW-DF-optPBE functional behaves similarly to PBE+D3 in this regard.

In Table S2, we show the computed Fe-N distances that we obtained for the FeP and FeP-Im models with the PBE+D3 and vdW-DF-optPBE functionals. The general trends we emphasize are: 1) the Fe-N distances increase with an increase in U-J, and 2) the Fe-N distances increase with an increased coordination number around $\mathrm{Fe}$; both of these trends have been discussed in the literature ${ }^{\mathrm{S} 1, \mathrm{~S} 2}$. The Fe$\mathrm{N}$ distances that were obtained with the vdW-DF-optPBE functional are, generally, slightly longer (by up to $0.01 \AA$ ) than those that were found with PBE+D3.

${ }^{S 1}$ D. A. Scherlis, M. Cococcioni, P. Sit, and N. Marzari. "Simulation of Heme Using DFT + U: A Step toward Accurate Spin-State Energetics” J. Phys. Chem. B (2007) 111, 7384-7391.

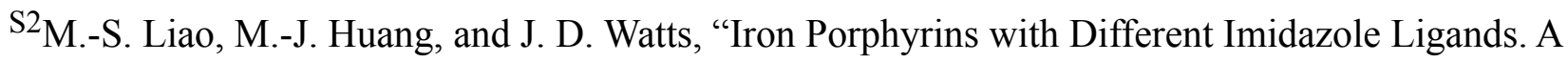
Theoretical Comparative Study” J. Phys. Chem. A, (2010) 114, 9554-9569. 


\begin{tabular}{|l|c|c|c|c|}
\hline Structure & $\mathbf{U}-\mathbf{J}$ & $\begin{array}{c}\Delta \mathbf{E}_{\mathbf{S}} \\
\text { (vdW-DF- } \\
\text { optPBE) }\end{array}$ & $\begin{array}{c}\Delta \mathbf{E}_{\mathbf{S}} \\
(\mathbf{P B E}+\mathbf{D 3})\end{array}$ & $\begin{array}{c}\boldsymbol{\Delta} \mathbf{E}_{\mathbf{S}} \\
(\mathbf{P B E})\end{array}$ \\
\hline FeP & $0 \mathrm{eV}$ & -17 & -17 & -16 \\
\hline FeP & $3 \mathrm{eV}$ & -3 & -1 & -1 \\
\hline FeP-Im & $3 \mathrm{eV}$ & +1 & +3 & +4 \\
\hline
\end{tabular}

Table S1: The computed energy differences between the lowest energy $\mathrm{S}=1$ and $\mathrm{S}=2$ electronic states that were found; $\Delta \mathrm{E}_{\mathrm{S}}$ is computed as $\mathrm{E}_{\mathrm{S}=1}-\mathrm{E}_{\mathrm{S}=2}$, and the FeP and FeP-Im models are shown in Figure S10. $\Delta \mathrm{E}_{\mathrm{S}}$ is shown for three functionals: $\mathrm{PBE}, \mathrm{PBE}+\mathrm{D} 3$, and vdW-DF-optPBE. The computed $\Delta \mathrm{E}_{\mathrm{S}}$ values that were computed without the $\mathrm{DFT}+\mathrm{U}$ method, ie. with $\mathrm{U}-\mathrm{J}=0 \mathrm{eV}$, are also shown.

\begin{tabular}{|l|c|c|c|c|c|}
\hline Structure & S & U-J & $\begin{array}{c}\text { Fe-N } \\
\text { (vdW-DF- } \\
\text { optPBE) }\end{array}$ & $\begin{array}{c}\text { Fe-N } \\
\text { (PBE+D3) }\end{array}$ & $\begin{array}{c}\text { Fe-N } \\
\text { (PBE) }\end{array}$ \\
\hline FeP & 1 & $0 \mathrm{eV}$ & 1.99 & 1.99 & 1.99 \\
\hline FeP & 2 & $0 \mathrm{eV}$ & 2.06 & 2.05 & 2.06 \\
\hline FeP & 1 & $3 \mathrm{eV}$ & 2.01 & 2.00 & 2.00 \\
\hline FeP & 2 & $3 \mathrm{eV}$ & 2.06 & 2.06 & 2.06 \\
\hline FeP-Im & 1 & $3 \mathrm{eV}$ & $2.02-2.03$ & $2.01-2.02$ & $2.02-2.02$ \\
\hline FeP-Im & 2 & $3 \mathrm{eV}$ & $2.08-2.09$ & $2.08-2.09$ & $2.08-2.09$ \\
\hline
\end{tabular}

Table S2: The ranges of computed Fe-N distances, expressed in $\AA$, in the lowest energy $\mathrm{S}=1$ and $\mathrm{S}=2$ electronic states that were found; the FeP and FeP-Im models are shown in Figure S10. For the FeP-Im model, only the $\mathrm{Fe}-\mathrm{N}$ distances within the $\mathrm{FeP}$ "fragment" are shown. We note that the range of values we see for the FeP-Im model come about from the 1-methylimidazole ligand (Im) breaking the symmetry of the chemical environment around it; in our model, the plane of the imidazole ring in the Im ligand was positioned to overlap with the straight $\mathrm{N}-\mathrm{Fe}-\mathrm{N}$ motif in the FeP fragment below it. 


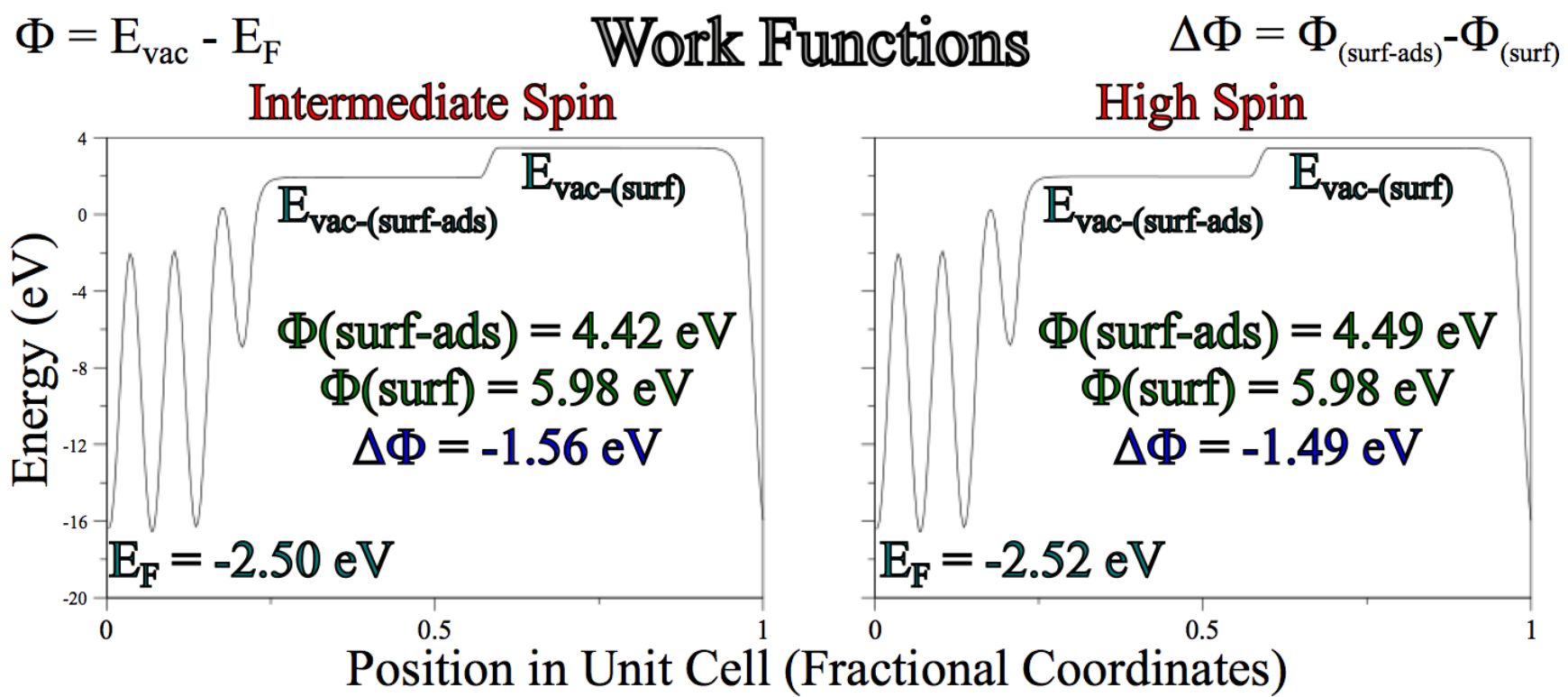

Figure S11: The planar averaged local potential taken along the $z$-axis as calculated with the vdW-DF-optPBE functional for the intermediate and high spin states of FeP adsorbed to the $\operatorname{Pt}(111)$ surface at the $B-45$ site. The change in the workfunction $(\Delta \Phi)$ was calculated by subtracting the workfunction of the surface $(\Phi($ surf $))$ from the workfunction of the surfaceadsorbate system ( $\Phi($ surf-ads)). The workfunction for each component was calculated via $\Phi=\mathbf{E}_{\mathrm{vac}}-\mathbf{E}_{\mathrm{F}}$. 
Section S9: Partial Charge Densities of the $S=1$ and $S=2$ FeP-Pt(111) Systems

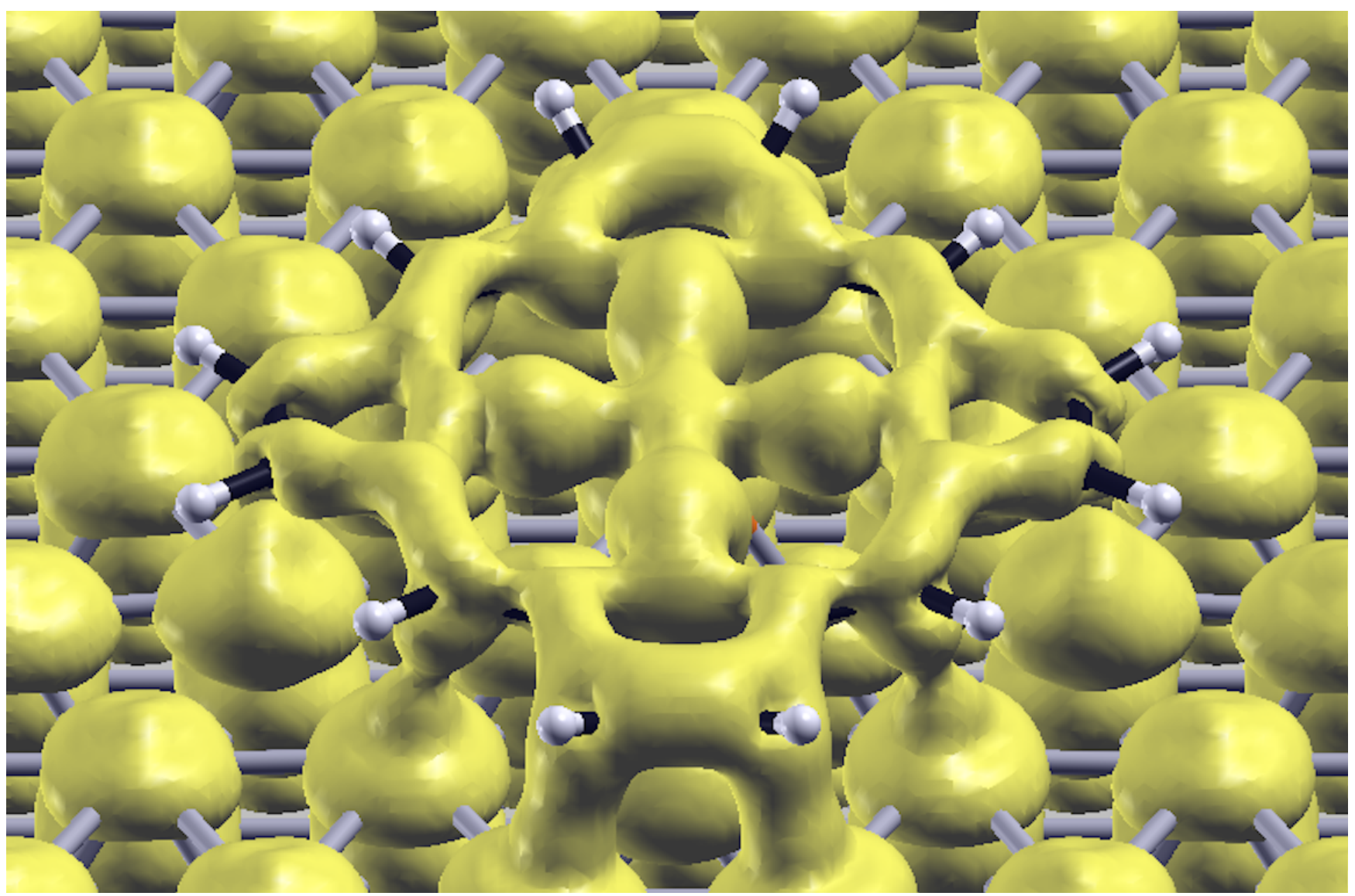

Figure S12: Partial charge density of the $S=1$ FeP-Pt(111) system at the energy range of $-5.00 \mathrm{eV}$ to $-4.25 \mathrm{eV}$. (isovalue $\left.=0.06 \mathrm{e} / \AA^{3}\right)$ 


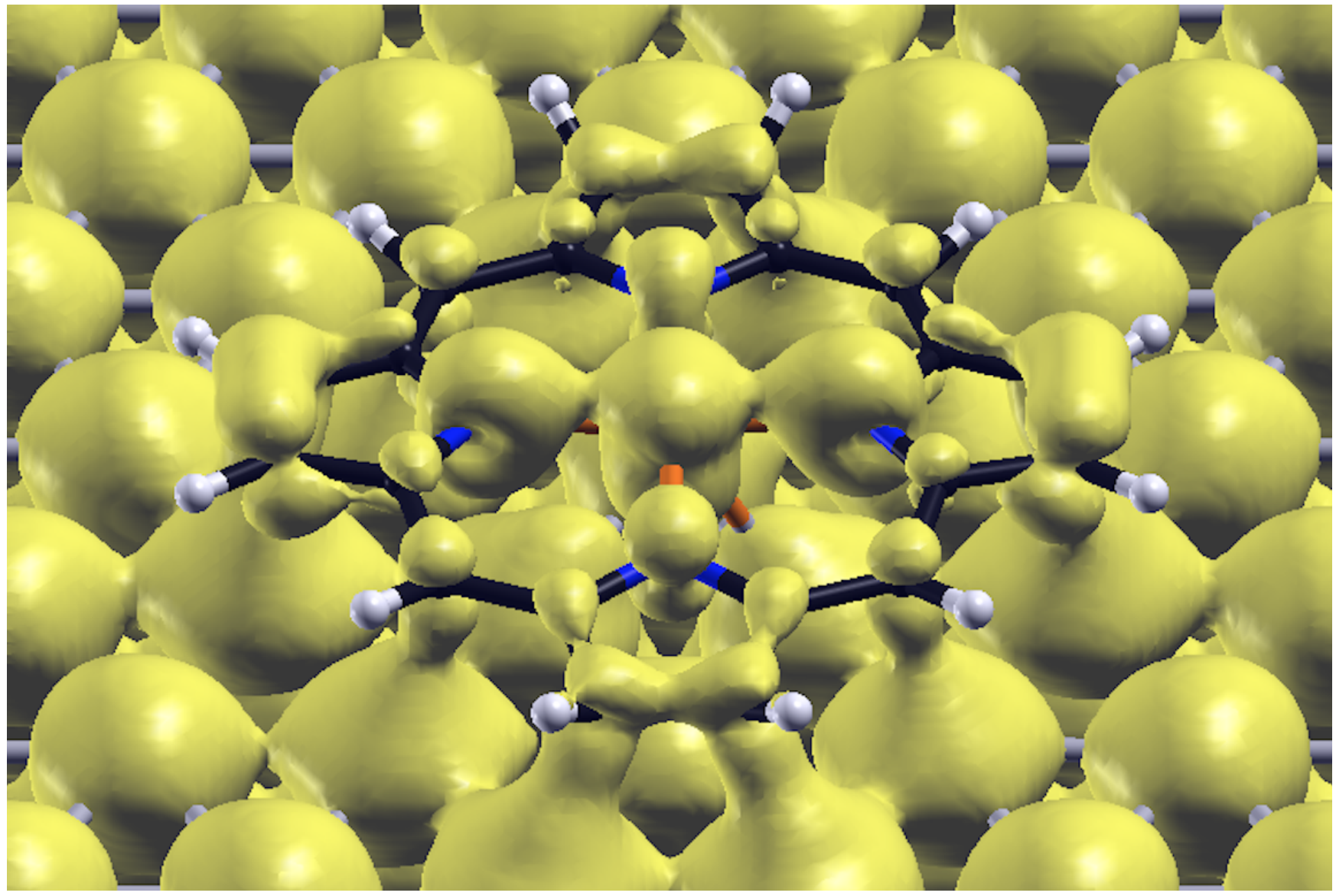

Figure S13: Partial charge density of the $S=1$ FeP-Pt(111) system at the energy range of $-4.20 \mathrm{eV}$ to $-3.25 \mathrm{eV}$. $\left(\right.$ isovalue $=0.06 \mathrm{e} / \AA^{3}$ ) 


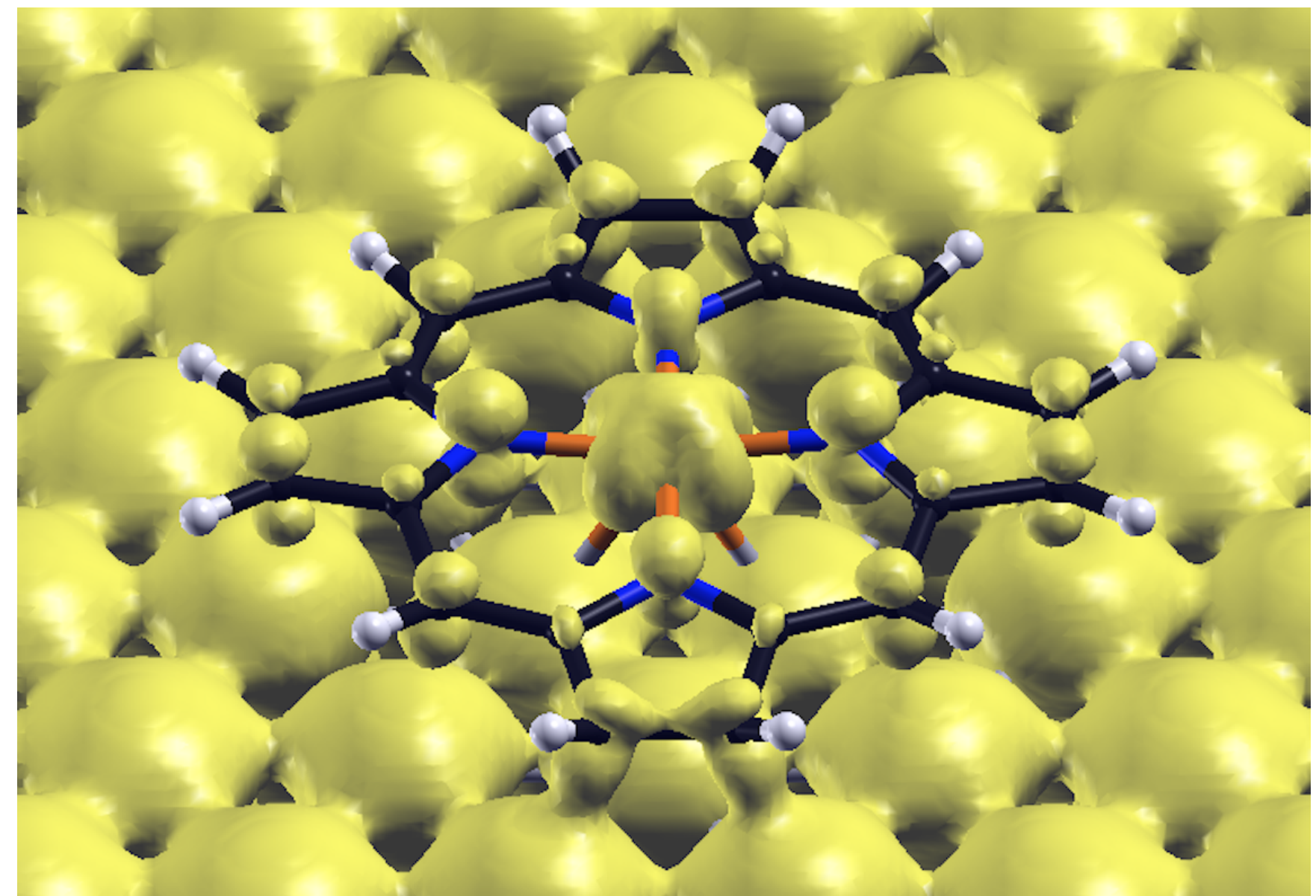

Figure S14: Partial charge density of the $S=1$ FeP-Pt(111) system at the energy range of $-3.20 \mathrm{eV}$ to $-2.30 \mathrm{eV}$. (isovalue $\left.=0.06 \mathrm{e} / \AA^{3}\right)$ 


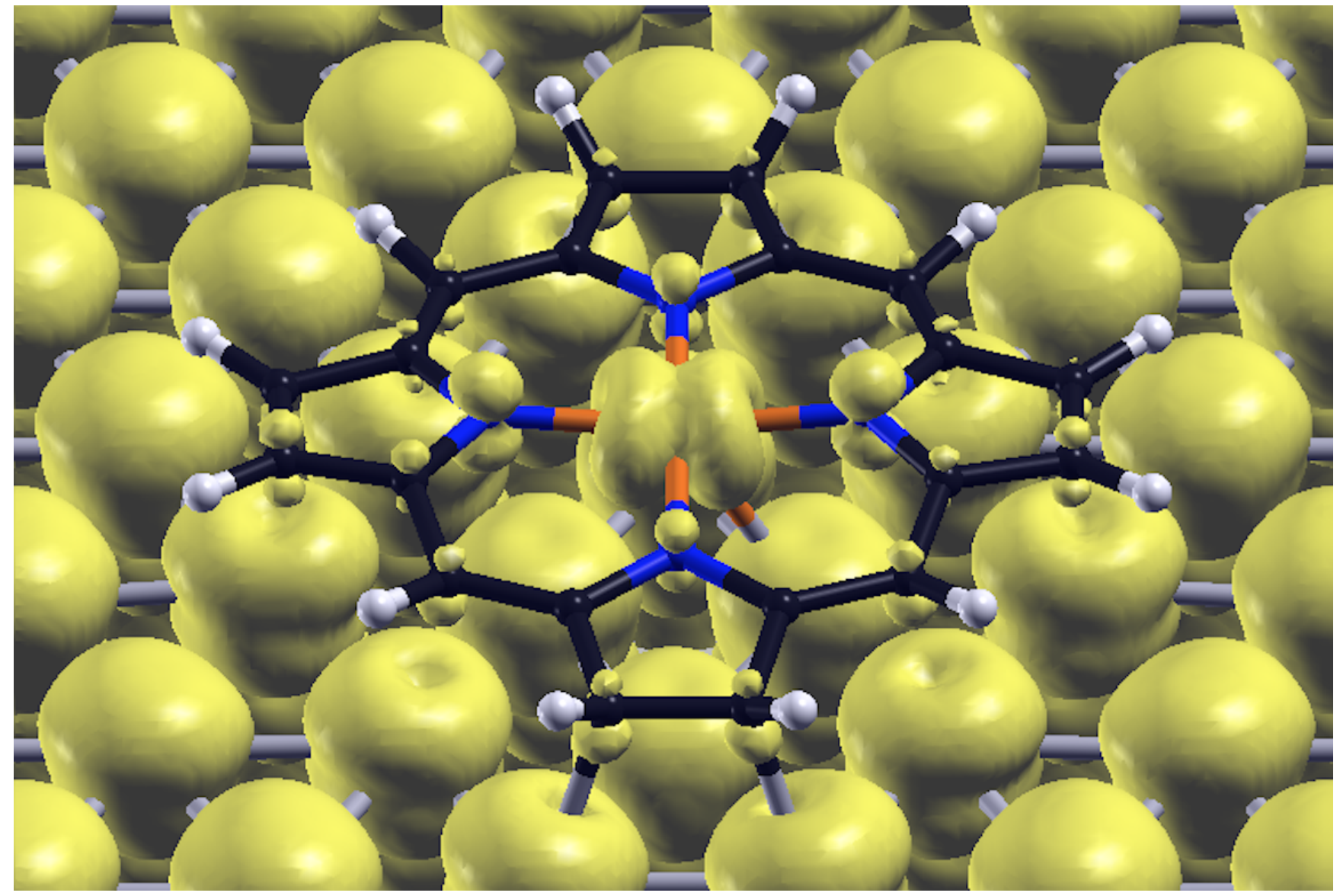

Figure S15: Partial charge density of the $S=1$ FeP-Pt(111) system at the energy range of $\mathbf{- 2 . 2 5} \mathrm{eV}$ to $-1.40 \mathrm{eV}$. (isovalue $=0.06 \mathrm{e} / \AA^{3}$ ) 


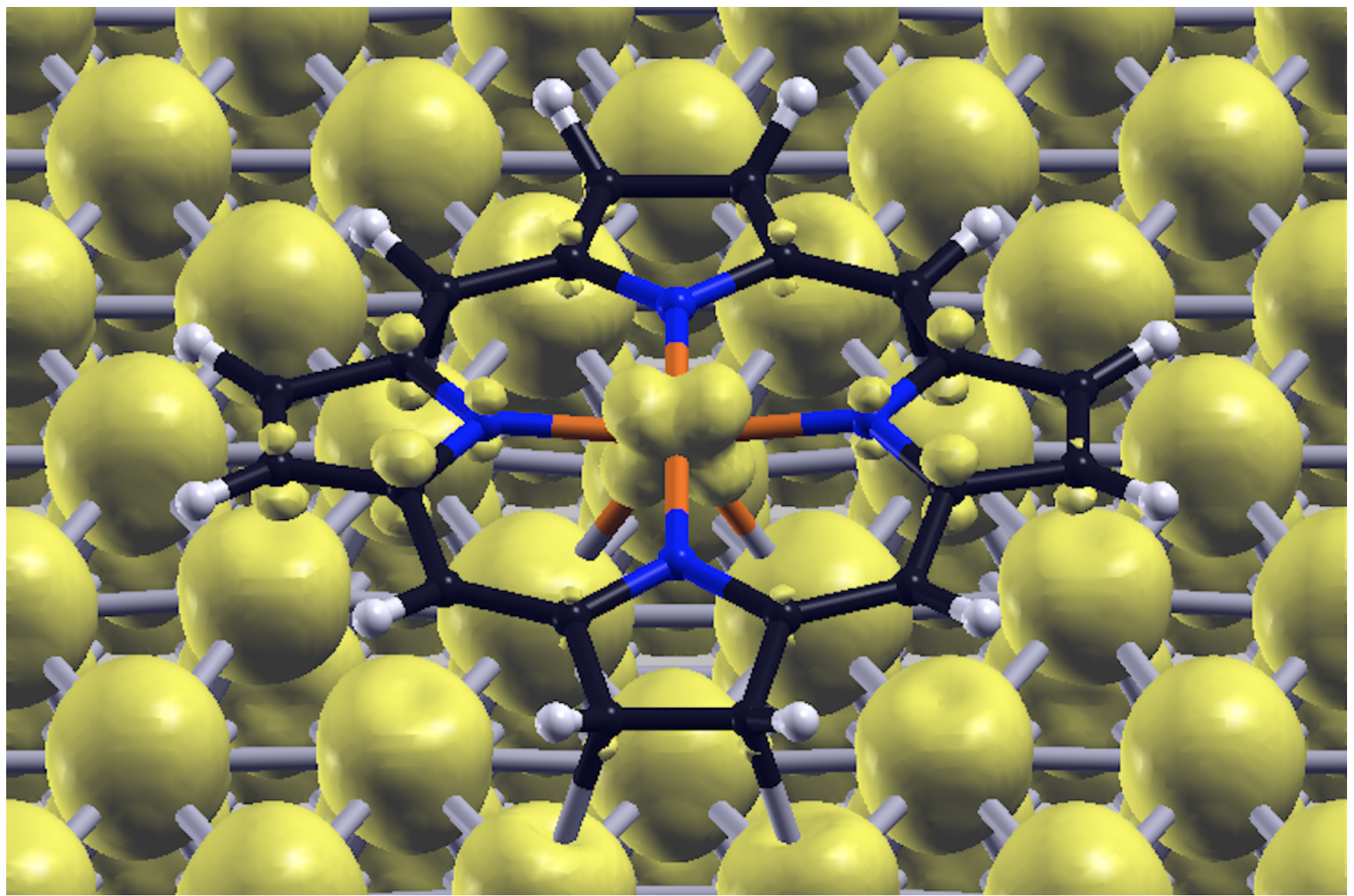

Figure S16: Partial charge density of the $S=1$ FeP-Pt(111) system at the energy range of $-1.20 \mathrm{eV}$ to $-0.50 \mathrm{eV}$. (isovalue $\left.=0.06 \mathrm{e} / \AA^{3}\right)$ 


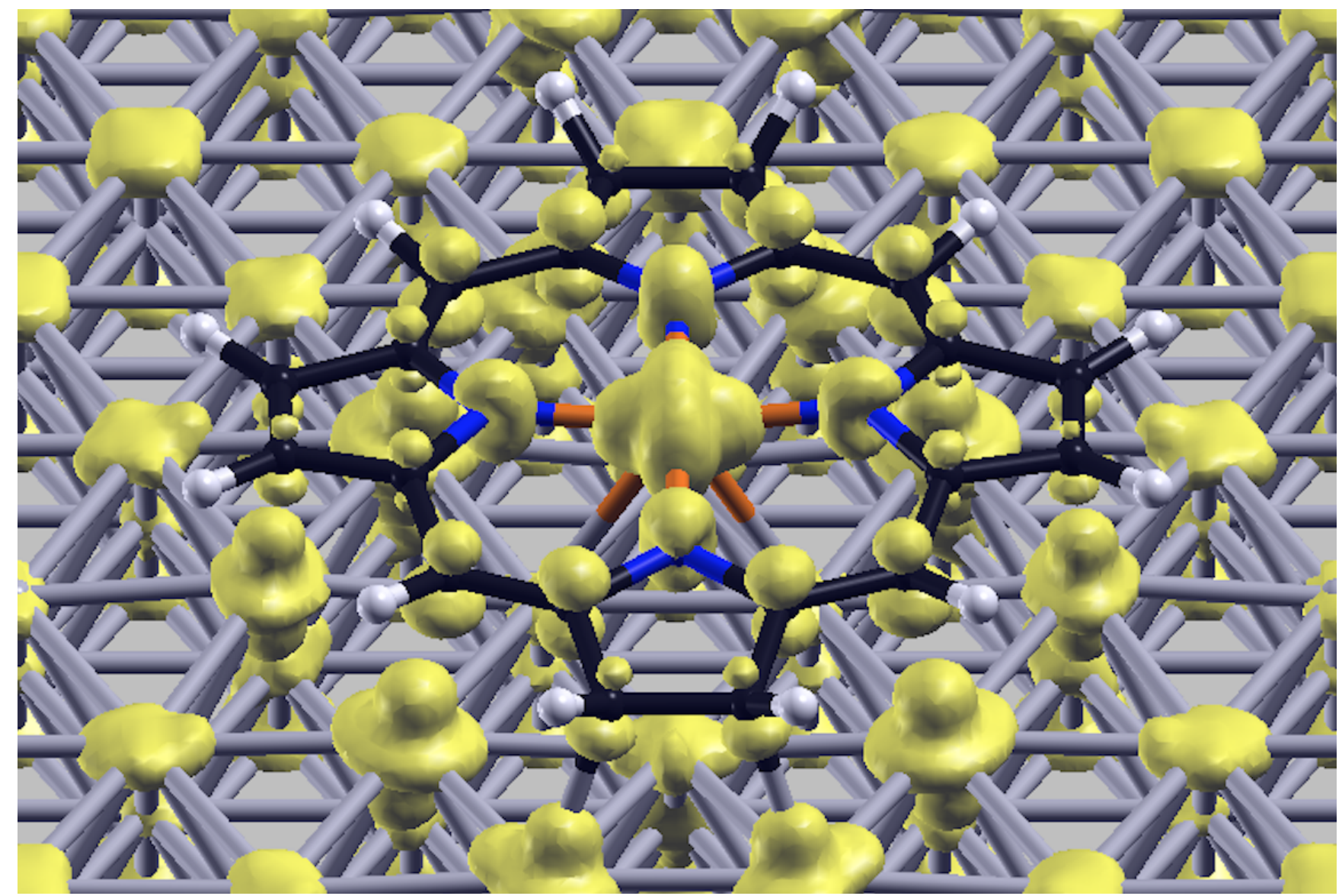

Figure S17: Partial charge density of the $S=1$ FeP-Pt(111) system at the energy range of $+0.50 \mathrm{eV}$ to $+1.30 \mathrm{eV}$. (isovalue $=0.06 \mathrm{e} / \AA^{3}$ ) 


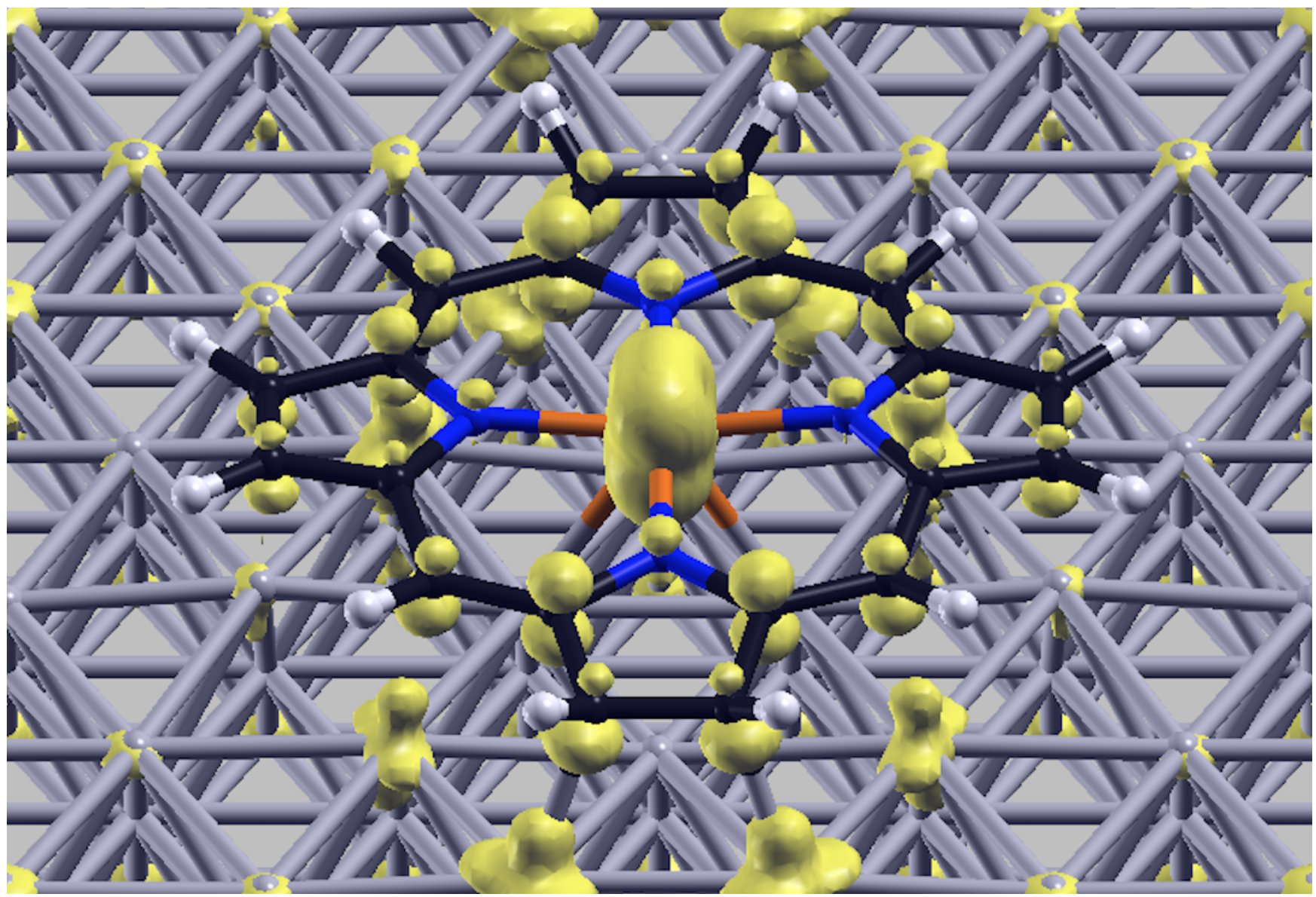

Figure S18: Partial charge density of the $S=1$ FeP-Pt(111) system at the energy range of $+1.40 \mathrm{eV}$ to $+2.10 \mathrm{eV}$. (isovalue $\left.=0.06 \mathrm{e} / \AA^{3}\right)$ 


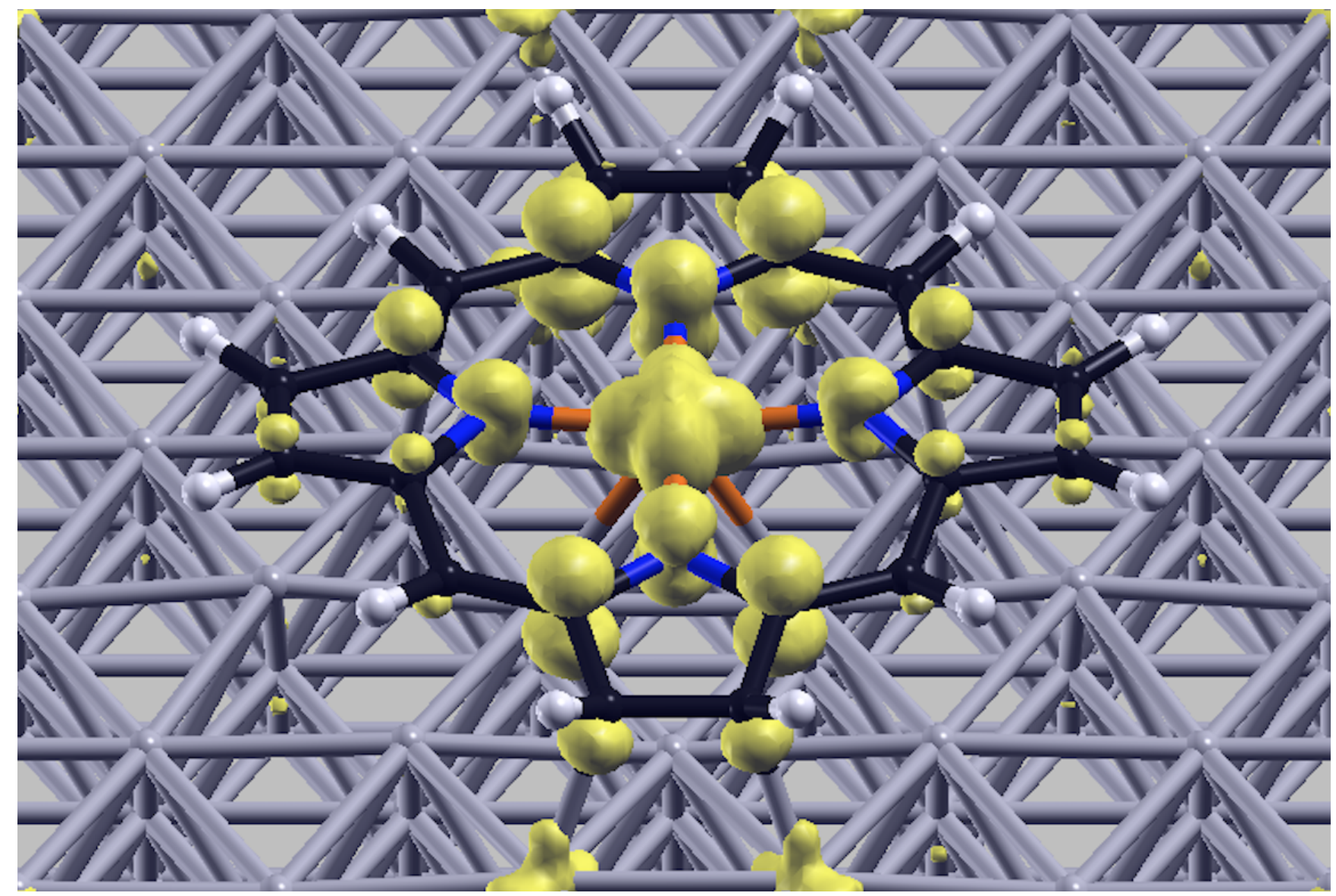

Figure S19: Partial charge density of the $S=1 \mathrm{FeP}-\mathrm{Pt}(111)$ system at the energy range of $+2.20 \mathrm{eV}$ to $+3.00 \mathrm{eV}$. (isovalue $\left.=0.06 \mathrm{e} / \AA^{3}\right)$ 


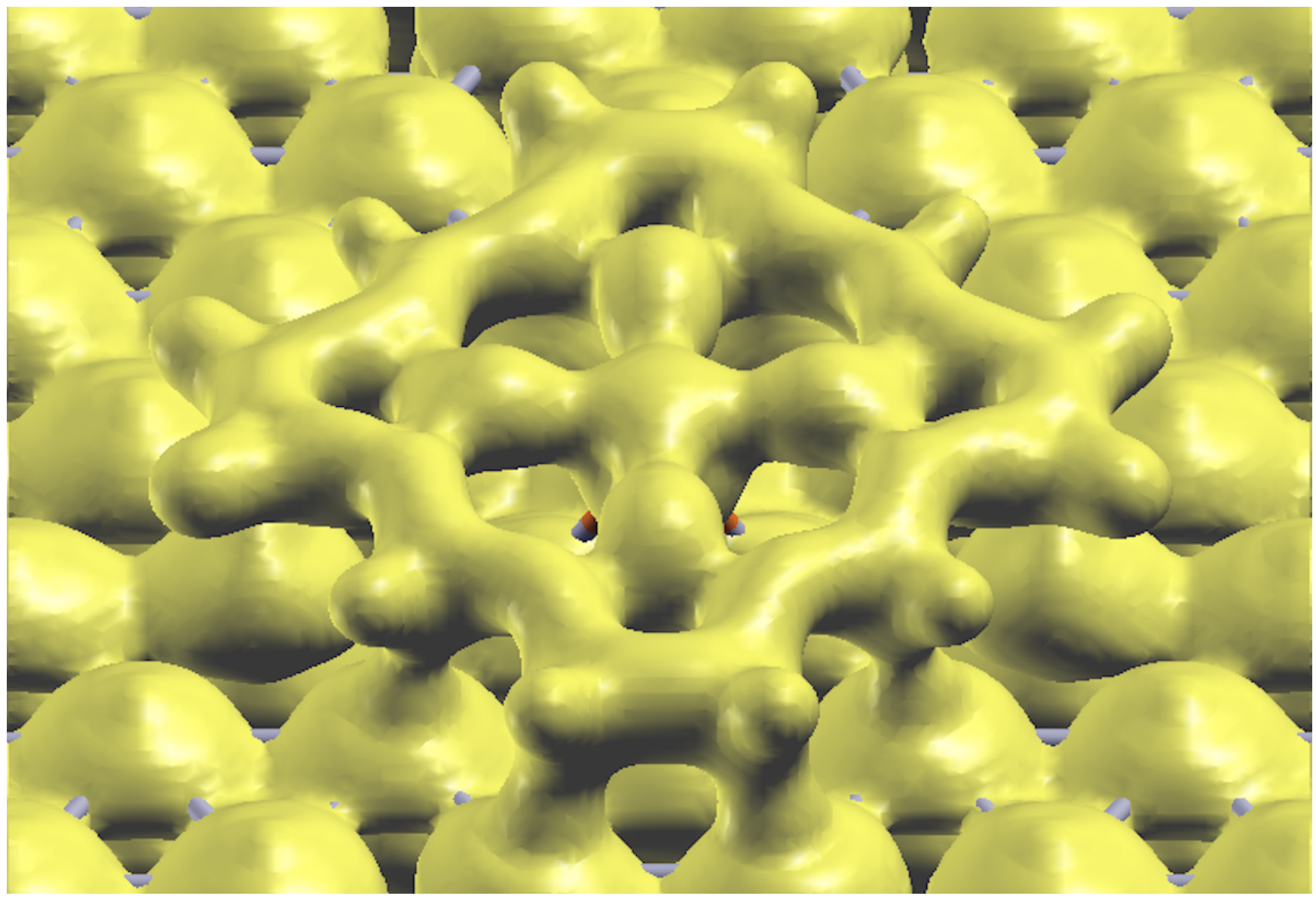

Figure S20: Partial charge density of the $S=2$ FeP-Pt(111) system at the energy range of $-6.00 \mathrm{eV}$ to $-4.25 \mathrm{eV}$. (isovalue $=0.1 \mathrm{e} / \AA^{3}$ ) 


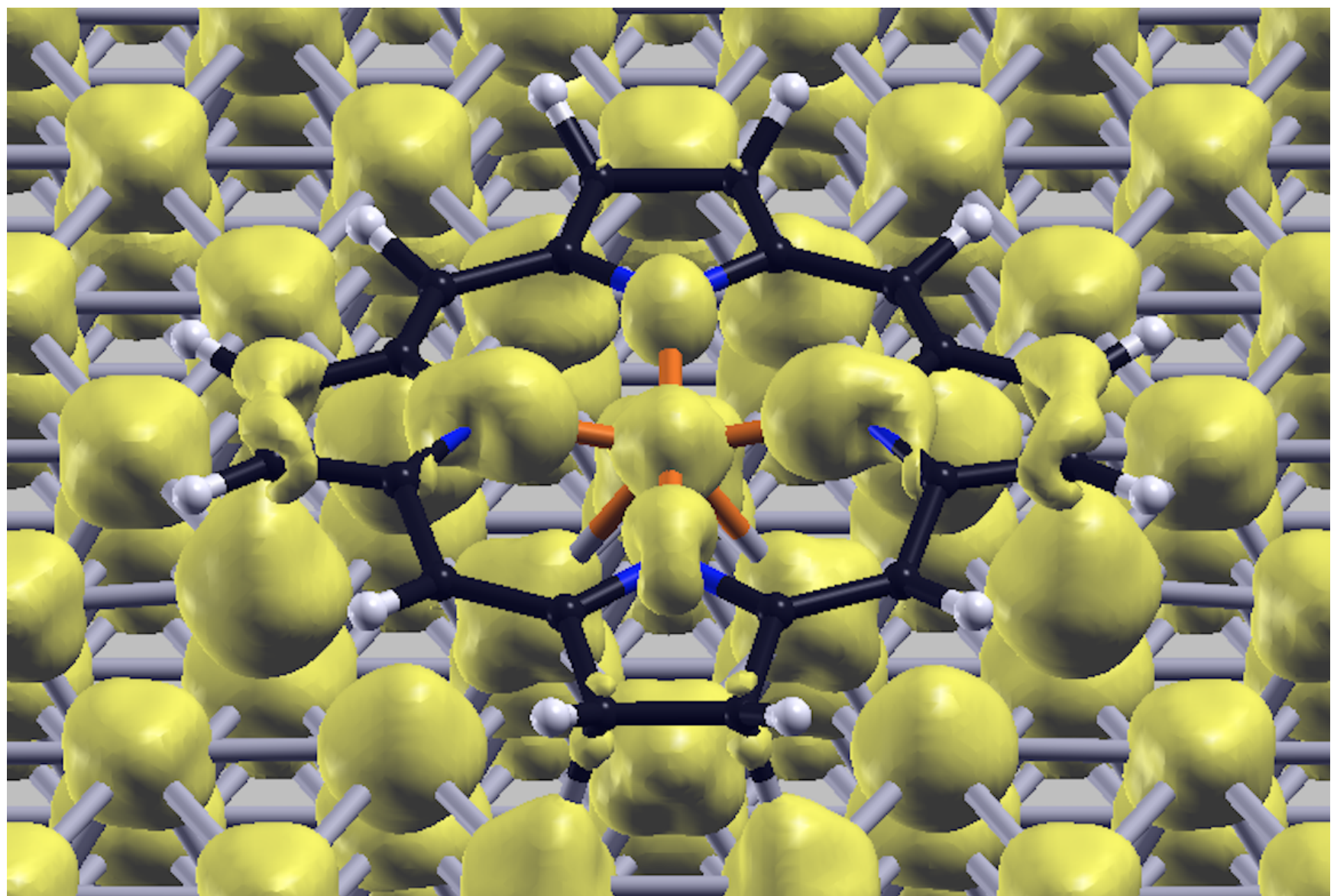

Figure S21: Partial charge density of the $S=2$ FeP-Pt(111) system at the energy range of $\mathbf{- 4 . 1 0} \mathrm{eV}$ to $-3.50 \mathrm{eV}$. (isovalue $=0.1 \mathrm{e} / \AA^{3}$ ) 


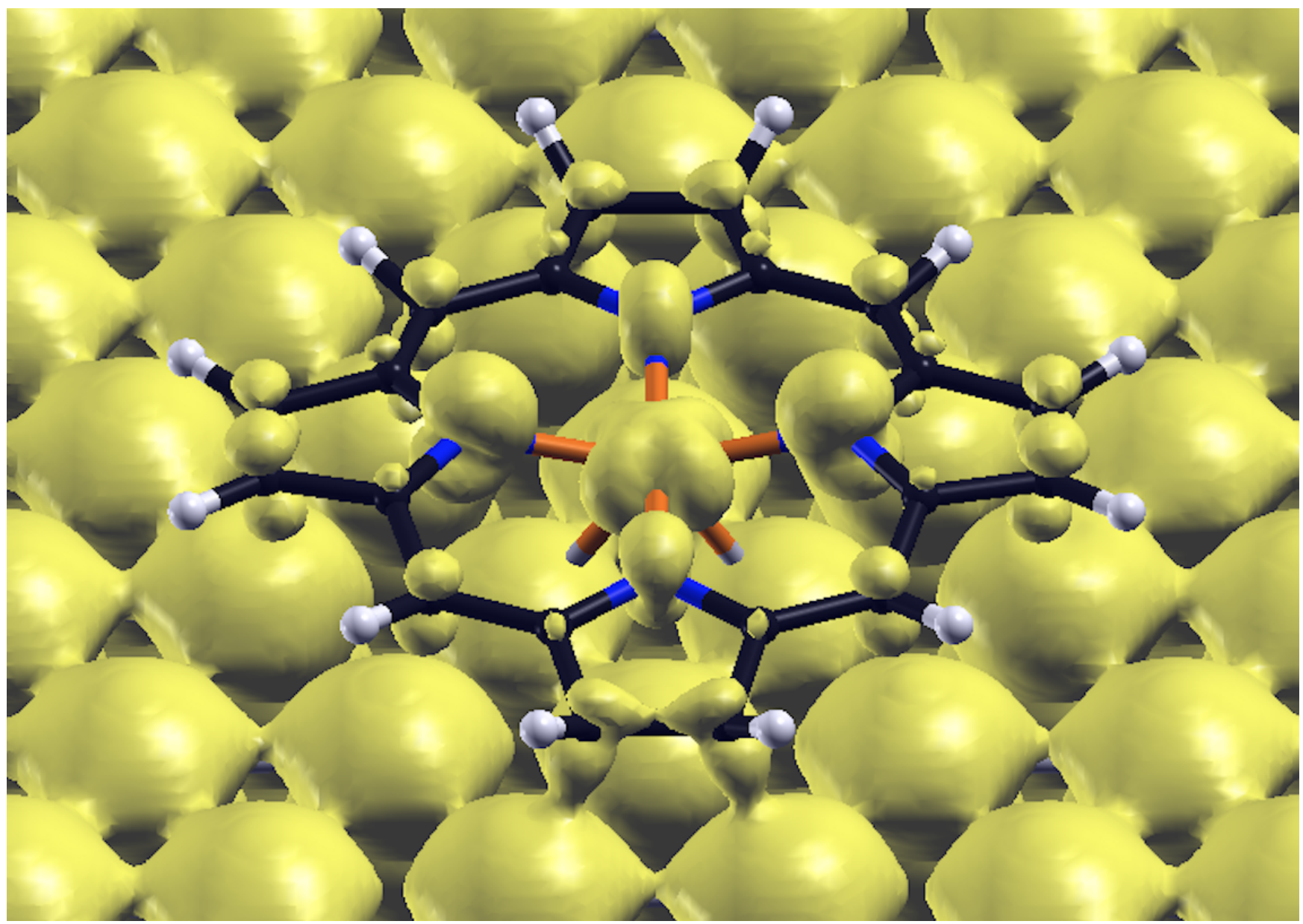

Figure S22: Partial charge density of the $S=2$ FeP-Pt(111) system at the energy range of $-3.45 \mathrm{eV}$ to $-2.10 \mathrm{eV}$. (isovalue $\left.=0.1 \mathrm{e} / \AA^{3}\right)$ 


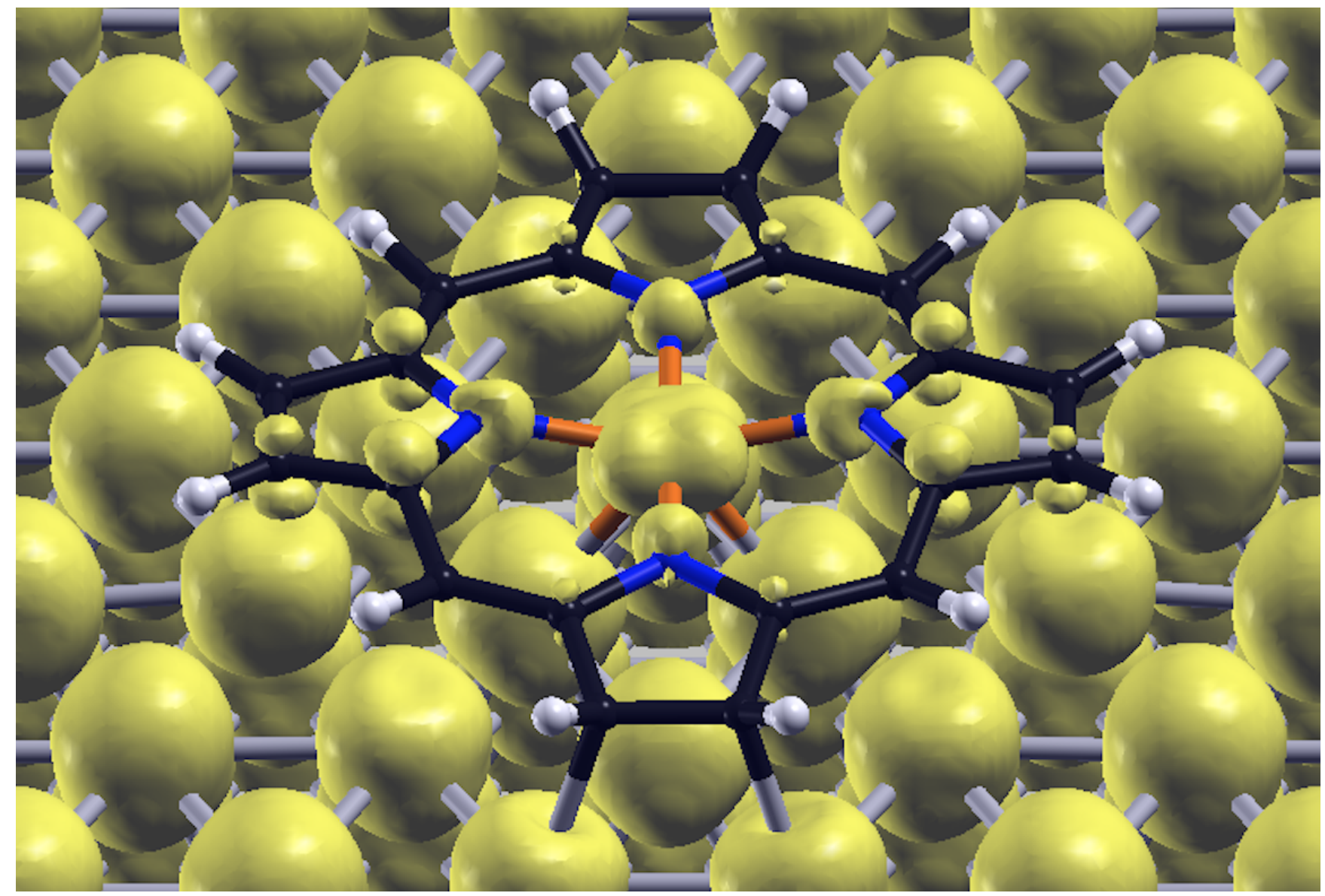

Figure S23: Partial charge density of the $S=2$ FeP-Pt(111) system at the energy range of $-1.40 \mathrm{eV}$ to $-0.30 \mathrm{eV}$. (isovalue $=0.1 \mathrm{e} / \AA^{3}$ ) 


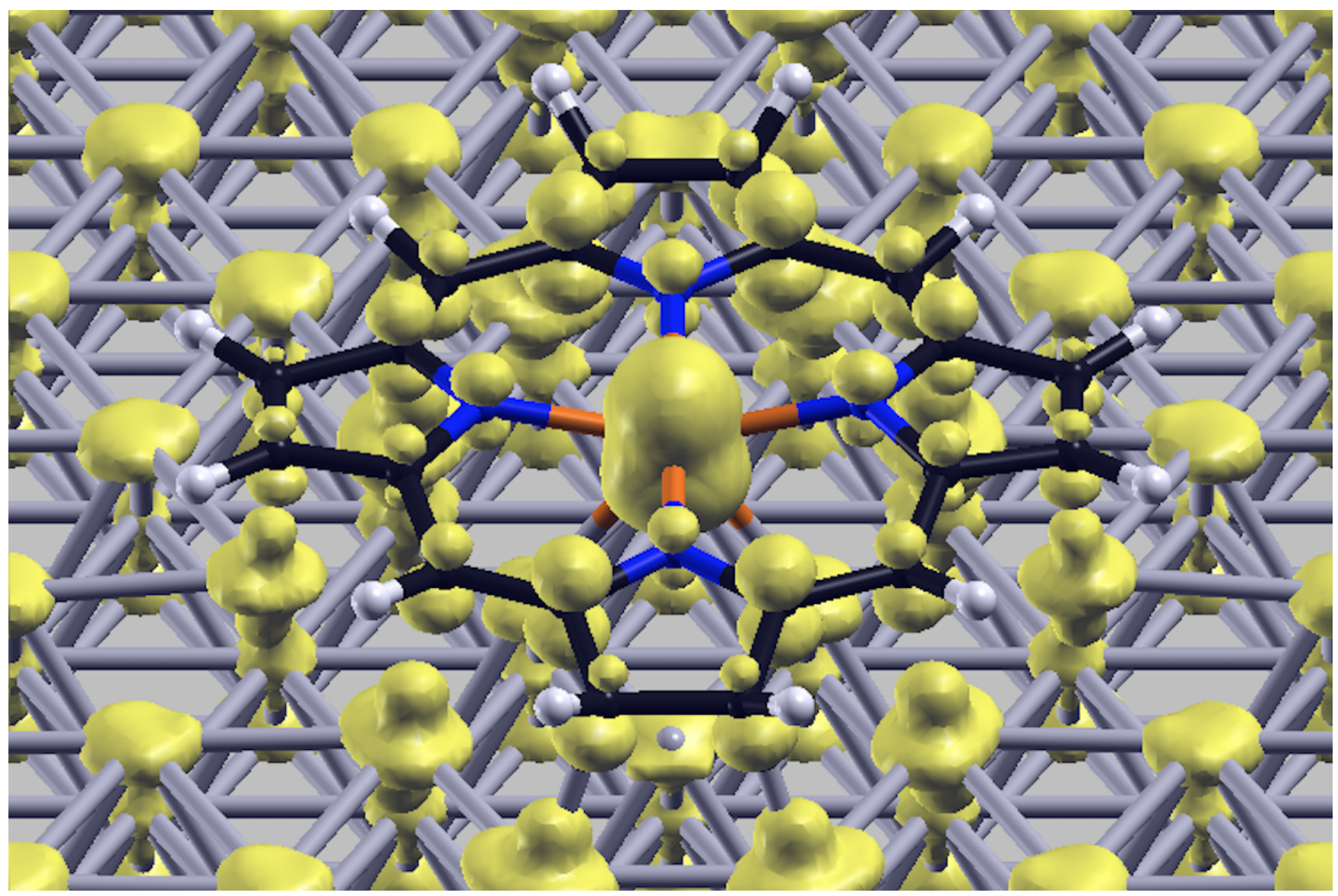

Figure S24: Partial charge density of the $S=2$ FeP-Pt(111) system at the energy range of $+1.00 \mathrm{eV}$ to $+2.60 \mathrm{eV}$. (isovalue $\left.=0.1 \mathrm{e} / \AA^{3}\right)$ 


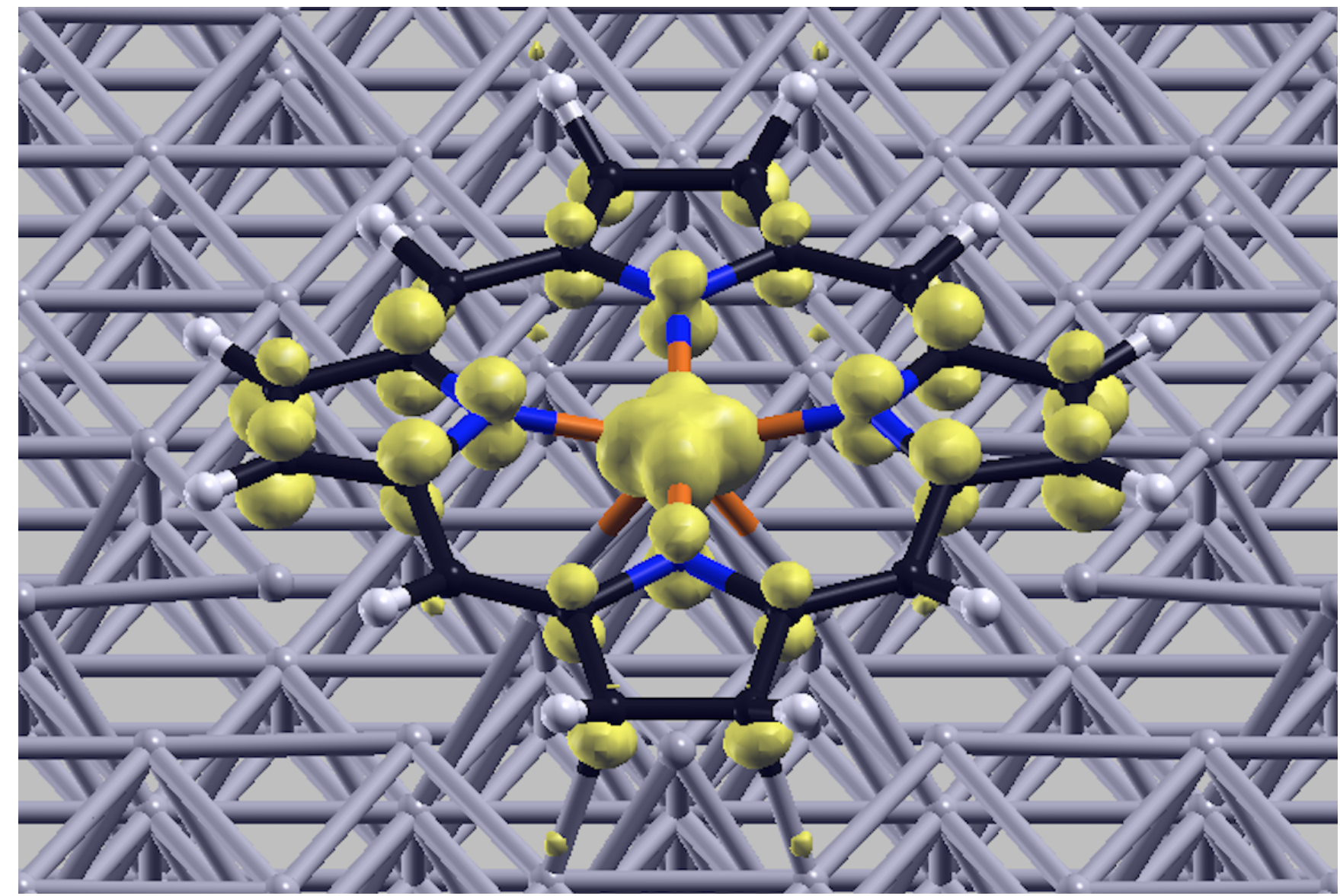

Figure S25: Partial charge density of the $\mathrm{S}=2 \mathrm{FeP}-\mathrm{Pt}(111)$ system at the energy range of $+3.50 \mathrm{eV}$ to $+4.40 \mathrm{eV}$. (isovalue $\left.=0.1 \mathrm{e} / \AA^{3}\right)$ 
Section S10: Coordinates of Isolated FeP and Pt(111) Surfaces (POSCAR format)

FeP - vdW-DF-optPBE, Ueff $=3, S=1$ :

$\mathrm{H} \mathrm{C} \mathrm{N} \mathrm{Fe}$

1.00000000000000

$\begin{array}{llll}14.1349999999999998 & 0.0000000000000000 & 0.0000000000000000\end{array}$

$\begin{array}{lll}0.0000000000000000 & 14.6880000000000006 & 0.0000000000000000\end{array}$

$\begin{array}{llll}0.0000000000000000 & 0.0000000000000000 & 35.0000000000000000\end{array}$

$\mathrm{H} \quad \mathrm{C} \quad \mathrm{N} \quad \mathrm{Fe}$

$\begin{array}{llll}12 & 20 & 4 & 1\end{array}$

Direct

$\begin{array}{llll}0.7191238659599293 & 0.4458740370534571 & 0.2267216543858623\end{array}$

$\begin{array}{llll}0.4002508376261602 & 0.1370679864871818 & 0.2272146451001689\end{array}$

$\begin{array}{llll}0.0789636272347281 & 0.4436168125424373 & 0.2270218773731898\end{array}$

$\begin{array}{llll}0.3978797808789665 & 0.7525241432282783 & 0.2269334739517603\end{array}$

$\begin{array}{llll}0.0762188186980879 & 0.6225390279048710 & 0.2278919002870339\end{array}$

$\begin{array}{llll}0.2118225208695250 & 0.7538120598700004 & 0.2278152546099648\end{array}$

$\begin{array}{llll}0.5836532296970844 & 0.7553354618969124 & 0.2280845472311910\end{array}$

$\begin{array}{llll}0.7201999419315257 & 0.6250423245027932 & 0.2280011036768030\end{array}$

$\begin{array}{llll}0.7216231212430628 & 0.2671312957958065 & 0.2275989014111275\end{array}$

$\begin{array}{llll}0.5862649039773160 & 0.1357535179439040 & 0.2276866550205128\end{array}$

$\begin{array}{llll}0.2143410572122377 & 0.1344126131757406 & 0.2284360448058393\end{array}$

$\begin{array}{llll}0.0778223602766843 & 0.2648313208316253 & 0.2285357369160081\end{array}$

$\begin{array}{llll}0.3110206352330067 & 0.6344967332085929 & 0.2263142019233584\end{array}$

$\begin{array}{llll}0.2205858413981261 & 0.6803965858995866 & 0.2270295029904119\end{array}$

$\begin{array}{lllll}0.1525433092298982 & 0.6144654959031115 & 0.2270713934208857\end{array}$

$\begin{array}{lllll}0.2010393544254825 & 0.5279088713055771 & 0.2263390476534184\end{array}$

$\begin{array}{llll}0.3981270523786336 & 0.6783395383512598 & 0.2264973746102257\end{array}$

$\begin{array}{llll}0.4855793834194060 & 0.6351722833309665 & 0.2264503068340034\end{array}$

$\begin{array}{llll}0.5962771685531081 & 0.5294072796635731 & 0.2262910852318569\end{array}$

$\begin{array}{lllll}0.6439163752763051 & 0.6164431750837238 & 0.2273587205008099\end{array}$

$\begin{array}{llll}0.5753642932559444 & 0.6818636452088356 & 0.2274609001207679\end{array}$

$\begin{array}{llll}0.6420549520071788 & 0.4456353276643696 & 0.2262048509327670\end{array}$

$\begin{array}{llll}0.5969247865732399 & 0.3615616104644204 & 0.2258991193860282\end{array}$

$\begin{array}{llll}0.4869856216423614 & 0.2550560004290290 & 0.2259838367213547\end{array}$

$\begin{array}{llll}0.5772778545102568 & 0.2091208760454819 & 0.2268806667410956\end{array}$

$\begin{array}{llll}0.6452703868998526 & 0.2750366966115863 & 0.2267259950224485\end{array}$

$\begin{array}{llll}0.3999535828559431 & 0.2112536438460069 & 0.2264950955000629\end{array}$

$\begin{array}{llll}0.3125387842217577 & 0.2544535057390291 & 0.2265077143785774\end{array}$

$\begin{array}{llll}0.2018254543382199 & 0.3602326181775695 & 0.2266182894170470\end{array}$

$\begin{array}{lllll}0.1560054529772685 & 0.4438717401254806 & 0.2265692661955967\end{array}$

$\begin{array}{llll}0.1541219393373012 & 0.2733538305566867 & 0.2277923278788379\end{array}$

$\begin{array}{llll}0.2226326842526944 & 0.2078952691448137 & 0.2276893607414507\end{array}$

$\begin{array}{llll}0.2981618977677059 & 0.5409696421422865 & 0.2258477532193837\end{array}$

$\begin{array}{llll}0.4991450039595975 & 0.5417370424410635 & 0.2257894182137647\end{array}$

$\begin{array}{lllll}0.2989926828761611 & 0.3478955040823877 & 0.2259067572798656\end{array}$

$\begin{array}{llll}0.4998594444579538 & 0.3485686787142939 & 0.2254251662445554\end{array}$ 
FeP - vdW-DF-optPBE, Ueff = 3, $S=2$ :

$\mathrm{H} \mathrm{C} \mathrm{N} \mathrm{Fe}$

1.00000000000000

$\begin{array}{lll}14.1349999999999998 & 0.0000000000000000 & 0.0000000000000000\end{array}$

$\begin{array}{llll}0.0000000000000000 & 14.6880000000000006 & 0.0000000000000000\end{array}$

$\begin{array}{llll}0.0000000000000000 & 0.0000000000000000 & 35.0000000000000000\end{array}$

$\begin{array}{llll}\mathrm{H} & \mathrm{C} & \mathrm{N} & \mathrm{Fe}\end{array}$

$\begin{array}{llll}12 & 20 & 4 & 1\end{array}$

Direct

$\begin{array}{llll}0.7198329552724516 & 0.4458948066700614 & 0.2267393620143920\end{array}$

$\begin{array}{llll}0.4001025840793844 & 0.1361617622828533 & 0.2272135802202513\end{array}$

$\begin{array}{llll}0.0781232918676480 & 0.4436668869830385 & 0.2270807789838258\end{array}$

$\begin{array}{llll}0.3976551664225454 & 0.7535070110108748 & 0.2269803551952521\end{array}$

$\begin{array}{llll}0.0746175705015162 & 0.6245131553605034 & 0.2278435282967877\end{array}$

$\begin{array}{lllll}0.2100213824849533 & 0.7556551128049307 & 0.2276885096069421\end{array}$

$\begin{array}{llll}0.5855400886430431 & 0.7571113799902562 & 0.2280848023466504\end{array}$

$\begin{array}{llll}0.7219766767542239 & 0.6268749209018267 & 0.2279445676460270\end{array}$

$\begin{array}{llll}0.7236624775392357 & 0.2650329640380349 & 0.2275005256126903\end{array}$

$\begin{array}{llll}0.5882192201187451 & 0.1338814615572872 & 0.2276114694185551\end{array}$

$\begin{array}{llll}0.2122193182433065 & 0.1327163420062831 & 0.2284350510458069\end{array}$

$\begin{array}{llll}0.0758498829005774 & 0.2629014782740171 & 0.2286036189473322\end{array}$

$\begin{array}{llll}0.3097369523715727 & 0.6362613309581704 & 0.2262598014414863\end{array}$

$\begin{array}{llll}0.2192950740955766 & 0.6823485873272830 & 0.2269718416320998\end{array}$

$\begin{array}{llll}0.1509086337547600 & 0.6161615104322848 & 0.2271584066141514\end{array}$

$\begin{array}{llll}0.1992006136123479 & 0.5293082971021263 & 0.2264111750725988\end{array}$

$\begin{array}{llll}0.3980510409109570 & 0.6793319310701449 & 0.2264845906430324\end{array}$

$\begin{array}{llll}0.4868912381354846 & 0.6370721599259852 & 0.2264926295469962\end{array}$

$\begin{array}{llll}0.5981262158332044 & 0.5308365396407382 & 0.2262583971781780\end{array}$

$\begin{array}{llll}0.6457494914791724 & 0.6179737369715781 & 0.2271690926348882\end{array}$

$\begin{array}{llll}0.5770069258596351 & 0.6836960413670070 & 0.2273100894127182\end{array}$

$\begin{array}{llll}0.6427299945856007 & 0.4456492278210433 & 0.2262359390651483\end{array}$

$\begin{array}{llll}0.5988903127844125 & 0.3602003867091952 & 0.2260716271612679\end{array}$

$\begin{array}{llll}0.4884429639665839 & 0.2530697738316761 & 0.2260683123161087\end{array}$

$\begin{array}{llll}0.5791043963361560 & 0.2072138243417783 & 0.2267900426570506\end{array}$

$\begin{array}{llll}0.6473951096762889 & 0.2733941118307328 & 0.2268199863392084\end{array}$

$\begin{array}{llll}0.3998952275797549 & 0.2103345480186221 & 0.2265333223925978\end{array}$

$\begin{array}{lllll}0.3110815372566407 & 0.2525664183063796 & 0.2266220791585098\end{array}$

$\begin{array}{llll}0.1998180061271455 & 0.3589135504593983 & 0.2266524474167539\end{array}$

$\begin{array}{lllll}0.1552243674889198 & 0.4439364391485014 & 0.2266403449851069\end{array}$

$\begin{array}{llll}0.1520680816504623 & 0.2718209773545510 & 0.2277694663974188\end{array}$

$\begin{array}{llll}0.2208741440885547 & 0.2060862447650180 & 0.2276589336936823\end{array}$

$\begin{array}{llll}0.2956091627660129 & 0.5434365169115409 & 0.2258375476704018\end{array}$

$\begin{array}{llll}0.5016496021858430 & 0.5443358368699975 & 0.2258251313079143\end{array}$

$\begin{array}{llll}0.2963003684727070 & 0.3453351955540498 & 0.2259651125931725\end{array}$

$\begin{array}{lllll}0.5024966543613161 & 0.3459359858153377 & 0.2254777871853193\end{array}$ 


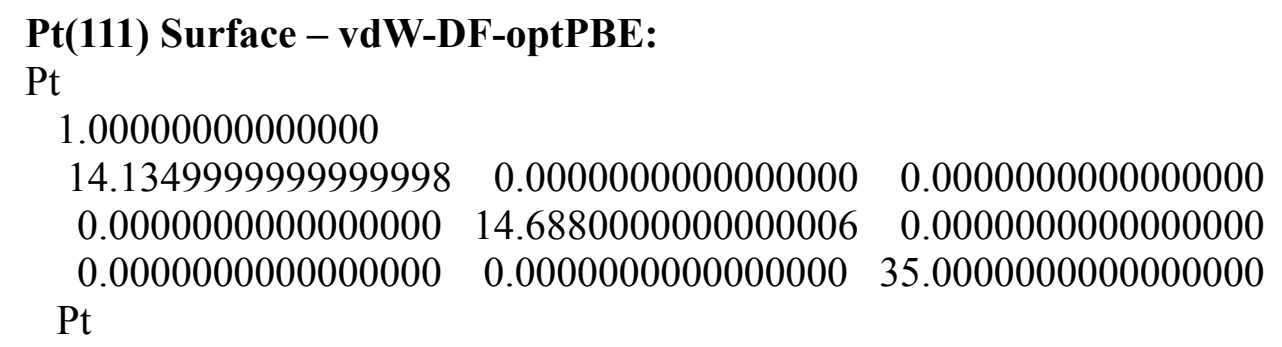

90

Direct

$\begin{array}{llll}0.6999029999999991 & 0.8333280000000016 & 0.0000000000000000\end{array}$

$\begin{array}{llll}0.7998899999999978 & 0.6666669999999968 & 0.0000000000000000\end{array}$

$\begin{array}{llll}0.5999169999999978 & 0.6666669999999968 & 0.0000000000000000\end{array}$

$\begin{array}{llll}0.8998759999999990 & 0.8333280000000016 & 0.0000000000000000\end{array}$

$\begin{array}{llll}0.2999590000000012 & 0.8333280000000016 & 0.0000000000000000\end{array}$

$\begin{array}{llll}0.3999450000000024 & 0.6666669999999968 & 0.0000000000000000\end{array}$

$\begin{array}{lllll}0.1999720000000025 & 0.6666669999999968 & 0.0000000000000000\end{array}$

$\begin{array}{llll}0.4999309999999966 & 0.8333280000000016 & 0.0000000000000000\end{array}$

$\begin{array}{llll}0.0000000000000000 & 0.6666669999999968 & 0.0000000000000000\end{array}$

$\begin{array}{llll}0.0999860000000012 & 0.8333280000000016 & 0.0000000000000000\end{array}$

$\begin{array}{lllll}0.6999029999999991 & 0.1666609999999977 & 0.0000000000000000\end{array}$

$\begin{array}{llll}0.7998899999999978 & 0.0000000000000000 & 0.0000000000000000\end{array}$

$\begin{array}{llll}0.5999169999999978 & 0.0000000000000000 & 0.0000000000000000\end{array}$

$\begin{array}{llll}0.8998759999999990 & 0.4999830000000003 & 0.0000000000000000\end{array}$

$\begin{array}{lllll}0.8998759999999990 & 0.1666609999999977 & 0.0000000000000000\end{array}$

$\begin{array}{llll}0.7998899999999978 & 0.3333220000000026 & 0.0000000000000000\end{array}$

$\begin{array}{llll}0.2999590000000012 & 0.1666609999999977 & 0.0000000000000000\end{array}$

$\begin{array}{llll}0.3999450000000024 & 0.0000000000000000 & 0.0000000000000000\end{array}$

$\begin{array}{llll}0.1999720000000025 & 0.0000000000000000 & 0.0000000000000000\end{array}$

$\begin{array}{llll}0.4999309999999966 & 0.4999830000000003 & 0.0000000000000000\end{array}$

$\begin{array}{lllll}0.4999309999999966 & 0.1666609999999977 & 0.0000000000000000\end{array}$

$\begin{array}{llll}0.5999169999999978 & 0.3333220000000026 & 0.0000000000000000\end{array}$

$\begin{array}{lll}0.3999450000000024 & 0.3333220000000026 & 0.0000000000000000\end{array}$

$\begin{array}{llll}0.6999029999999991 & 0.4999830000000003 & 0.0000000000000000\end{array}$

$\begin{array}{lllll}0.0000000000000000 & 0.0000000000000000 & 0.0000000000000000\end{array}$

$\begin{array}{llll}0.0999860000000012 & 0.4999830000000003 & 0.0000000000000000\end{array}$

$\begin{array}{lllll}0.0999860000000012 & 0.1666609999999977 & 0.0000000000000000\end{array}$

$\begin{array}{llll}0.1999720000000025 & 0.3333220000000026 & 0.0000000000000000\end{array}$

$\begin{array}{llll}0.0000000000000000 & 0.3333220000000026 & 0.0000000000000000\end{array}$

$\begin{array}{llll}0.2999590000000012 & 0.4999830000000003 & 0.0000000000000000\end{array}$

$\begin{array}{llll}0.8998759999999990 & 0.7222200000000001 & 0.0659410285714301\end{array}$

$\begin{array}{lllll}0.4999309999999966 & 0.7222200000000001 & 0.0659410285714301\end{array}$

$\begin{array}{lllll}0.6999029999999991 & 0.7222200000000001 & 0.0659410285714301\end{array}$

$\begin{array}{lllll}0.7998899999999978 & 0.8888809999999978 & 0.0659410285714301\end{array}$

$\begin{array}{lllll}0.5999169999999978 & 0.8888809999999978 & 0.0659410285714301\end{array}$

$\begin{array}{lllll}0.0999860000000012 & 0.7222200000000001 & 0.0659410285714301\end{array}$ 
$\begin{array}{llll}0.2999590000000012 & 0.7222200000000001 & 0.0659410285714301\end{array}$ $\begin{array}{lllll}0.3999450000000024 & 0.8888809999999978 & 0.0659410285714301\end{array}$ $\begin{array}{llll}0.1999720000000025 & 0.8888809999999978 & 0.0659410285714301\end{array}$ $\begin{array}{lllll}0.0000000000000000 & 0.8888809999999978 & 0.0659410285714301\end{array}$ $\begin{array}{llll}0.8998759999999990 & 0.0555540000000008 & 0.0659410285714301\end{array}$ $\begin{array}{llll}0.4999309999999966 & 0.0555540000000008 & 0.0659410285714301\end{array}$ $\begin{array}{lllll}0.6999029999999991 & 0.3888749999999987 & 0.0659410285714301\end{array}$ $\begin{array}{llll}0.6999029999999991 & 0.0555540000000008 & 0.0659410285714301\end{array}$ $\begin{array}{llll}0.7998899999999978 & 0.2222149999999985 & 0.0659410285714301\end{array}$ $\begin{array}{llll}0.5999169999999978 & 0.2222149999999985 & 0.0659410285714301\end{array}$ $\begin{array}{llll}0.8998759999999990 & 0.3888749999999987 & 0.0659410285714301\end{array}$ $\begin{array}{llll}0.7998899999999978 & 0.5555360000000036 & 0.0659410285714301\end{array}$ $\begin{array}{llll}0.0999860000000012 & 0.0555540000000008 & 0.0659410285714301\end{array}$ $\begin{array}{llll}0.2999590000000012 & 0.3888749999999987 & 0.0659410285714301\end{array}$ $\begin{array}{llll}0.2999590000000012 & 0.0555540000000008 & 0.0659410285714301\end{array}$ $\begin{array}{llll}0.3999450000000024 & 0.2222149999999985 & 0.0659410285714301\end{array}$ $\begin{array}{llll}0.1999720000000025 & 0.2222149999999985 & 0.0659410285714301\end{array}$ $\begin{array}{lllll}0.4999309999999966 & 0.3888749999999987 & 0.0659410285714301\end{array}$ $\begin{array}{llll}0.5999169999999978 & 0.5555360000000036 & 0.0659410285714301\end{array}$ $\begin{array}{llll}0.3999450000000024 & 0.5555360000000036 & 0.0659410285714301\end{array}$ $\begin{array}{llll}0.0000000000000000 & 0.2222149999999985 & 0.0659410285714301\end{array}$ $\begin{array}{lllll}0.0999860000000012 & 0.3888749999999987 & 0.0659410285714301\end{array}$ $\begin{array}{llll}0.1999720000000025 & 0.5555360000000036 & 0.0659410285714301\end{array}$ $\begin{array}{llll}0.0000000000000000 & 0.5555360000000036 & 0.0659410285714301\end{array}$ $\begin{array}{llll}0.8999864530678298 & 0.9430179569745333 & 0.1325988511518165\end{array}$ $\begin{array}{llll}0.7999716810408403 & 0.7763710562574289 & 0.1323526151560627\end{array}$ $\begin{array}{llll}0.4999614802335310 & 0.9432372762783799 & 0.1325565385629108\end{array}$ $\begin{array}{llll}0.5999338741508011 & 0.7764161683917596 & 0.1323511498307774\end{array}$ $\begin{array}{llll}0.3999074732700764 & 0.7764235513510550 & 0.1323223138266643\end{array}$ $\begin{array}{llll}0.6999867045847602 & 0.9430292257059780 & 0.1326058955617932\end{array}$ $\begin{array}{llll}0.0999766290019247 & 0.9430493187155118 & 0.1326091424977907\end{array}$ $\begin{array}{llll}0.1999490048911478 & 0.7763764965877522 & 0.1323378836854807\end{array}$ $\begin{array}{llll}0.9999418650589251 & 0.7763502984120816 & 0.1323370904255489\end{array}$ $\begin{array}{llll}0.2999262657717218 & 0.9431284291730665 & 0.1325878141441066\end{array}$ $\begin{array}{llll}0.8999241783488843 & 0.2764155074352540 & 0.1323100244384378\end{array}$ $\begin{array}{llll}0.7999803451471845 & 0.1094748015476950 & 0.1325830257338009\end{array}$ $\begin{array}{llll}0.4998137792728188 & 0.2770353355778575 & 0.1324748155167324\end{array}$ $\begin{array}{llll}0.5999285765133422 & 0.1096419665965900 & 0.1325298414929430\end{array}$ $\begin{array}{llll}0.3999323247614787 & 0.1097370084691574 & 0.1325006730343373\end{array}$ $\begin{array}{llll}0.6999178409695301 & 0.6095074380588348 & 0.1325572712538958\end{array}$ $\begin{array}{llll}0.6997653211384969 & 0.2764763984685077 & 0.1323122011085242\end{array}$ $\begin{array}{llll}0.7996576751722978 & 0.4429631143874734 & 0.1325650796918458\end{array}$ $\begin{array}{llll}0.5994955093493122 & 0.4433279924183806 & 0.1326179445244998\end{array}$ $\begin{array}{llll}0.8999588464326579 & 0.6094326243656608 & 0.1325376974723238\end{array}$ $\begin{array}{llll}0.1001535659468949 & 0.2765005739125684 & 0.1323099164448778\end{array}$ $\begin{array}{llll}0.2000480979184416 & 0.1096269984810794 & 0.1325244166543654\end{array}$ $\begin{array}{llll}1.0000101384996423 & 0.1095368743732209 & 0.1325394548346460\end{array}$ 
$\begin{array}{llll}0.2999643890628914 & 0.6095178078445208 & 0.1325855338548241\end{array}$

$\begin{array}{llll}0.3000675158229038 & 0.2770712480372001 & 0.1324882944413370\end{array}$

$\begin{array}{llll}0.3999360671679700 & 0.4432472308603601 & 0.1326220160052143\end{array}$

$\begin{array}{llll}0.2002281282728917 & 0.4432708325817875 & 0.1326275442819182\end{array}$

$\begin{array}{llll}0.4999127478847520 & 0.6095915240868383 & 0.1325693782548400\end{array}$

$\begin{array}{llll}0.0000143832965594 & 0.4430381017087038 & 0.1325539429109374\end{array}$

$\begin{array}{llll}0.1000008213410234 & 0.6094866004629617 & 0.1325655143338723\end{array}$

FeP - vdW-DF-optB88, Ueff $=3$ :

$\mathrm{H} \mathrm{C} \mathrm{N} \mathrm{Fe}$

1.00000000000000

$\begin{array}{llll}14.0790000000000006 & 0.0000000000000000 & 0.0000000000000000\end{array}$

$\begin{array}{llll}0.0000000000000000 & 14.6310000000000002 & 0.0000000000000000\end{array}$

$\begin{array}{llll}0.0000000000000000 & 0.0000000000000000 & 35.0000000000000000\end{array}$

$\mathrm{H} \quad \mathrm{C} \quad \mathrm{N} \quad \mathrm{Fe}$

$\begin{array}{llll}12 & 20 & 4 & 1\end{array}$

Direct

$\begin{array}{llll}0.7198393216573634 & 0.4458296712243567 & 0.2268223052023599\end{array}$

$\begin{array}{llll}0.4001145124481293 & 0.1362328597967334 & 0.2273667801428064\end{array}$

$\begin{array}{llll}0.0783108441321707 & 0.4436542825808039 & 0.2272686387354749\end{array}$

$\begin{array}{llll}0.3979949869625044 & 0.7534564855268246 & 0.2271215065434013\end{array}$

$\begin{array}{llll}0.0756254256601923 & 0.6228144060148272 & 0.2281075433243765\end{array}$

$\begin{array}{llll}0.2116959063335704 & 0.7546287175447638 & 0.2280514228262023\end{array}$

$\begin{array}{llll}0.5840765396200723 & 0.7560204029776787 & 0.2281404480589632\end{array}$

$\begin{array}{llll}0.7209981123760759 & 0.6251285541372226 & 0.2280558161849558\end{array}$

$\begin{array}{llll}0.7222119832748071 & 0.2666684148163070 & 0.2278543604167306\end{array}$

$\begin{array}{llll}0.5863907174792651 & 0.1348994835756509 & 0.2278759996153412\end{array}$

$\begin{array}{lllll}0.2140293731146891 & 0.1336963767597437 & 0.2285325764585361\end{array}$

$\begin{array}{llll}0.0769843582151183 & 0.2646139912129292 & 0.2285339809448460\end{array}$

$\begin{array}{lllll}0.3110424695821905 & 0.6347782298430774 & 0.2262053245530615\end{array}$

$\begin{array}{llll}0.2206362898733036 & 0.6808902406354738 & 0.2271829339066744\end{array}$

$\begin{array}{llll}0.1523319808357012 & 0.6146907709631824 & 0.2271695292682287\end{array}$

$\begin{array}{llll}0.2010167781419668 & 0.5282148365002866 & 0.2261682452795572\end{array}$

$\begin{array}{llll}0.3982000228895615 & 0.6788994169727431 & 0.2264836537205416\end{array}$

$\begin{array}{lllll}0.4857871673617917 & 0.6353917729182134 & 0.2263408968954894\end{array}$

$\begin{array}{llll}0.5964338315295085 & 0.5295177245256135 & 0.2262555892226055\end{array}$

$\begin{array}{llll}0.6443308115960324 & 0.6164853925781149 & 0.2273996680069709\end{array}$

$\begin{array}{llll}0.5756434982352699 & 0.6822085532663351 & 0.2274607671364325\end{array}$

$\begin{array}{llll}0.6423547824810494 & 0.4456008264836145 & 0.2262062173151696\end{array}$

$\begin{array}{lllll}0.5970143927006504 & 0.3613875889199381 & 0.2258943772547804\end{array}$

$\begin{array}{llll}0.4869224009177239 & 0.2547987725427699 & 0.2259090984402916\end{array}$

$\begin{array}{llll}0.5771930345106968 & 0.2086030311091749 & 0.2269483274369050\end{array}$

$\begin{array}{lllll}0.6454763671568911 & 0.2748069732740426 & 0.2269895541866092\end{array}$

$\begin{array}{llll}0.3997442215734685 & 0.2107853252157014 & 0.2264884518565876\end{array}$

$\begin{array}{lllll}0.3122987014060009 & 0.2542566335342218 & 0.2264543368713828\end{array}$

$\begin{array}{llll}0.2016799908304281 & 0.3601230535890664 & 0.2264289152085725\end{array}$

$\begin{array}{lllll}0.1557977900902309 & 0.4439660962837821 & 0.2265328148012092\end{array}$ 
$\begin{array}{llll}0.1536709459764463 & 0.2732778479306259 & 0.2277703807767522\end{array}$

$\begin{array}{llll}0.2223394802868641 & 0.2075460225498590 & 0.2277271896421759\end{array}$

$\begin{array}{llll}0.2981331653550462 & 0.5412712250799960 & 0.2254117870555377\end{array}$

$\begin{array}{llll}0.4992955993458515 & 0.5419534338714725 & 0.2256336212784608\end{array}$

$\begin{array}{llll}0.2988352732997004 & 0.3476701841156564 & 0.2255428434468708\end{array}$

$\begin{array}{llll}0.4999403885686478 & 0.3483182989522399 & 0.2252423745141533\end{array}$

$\begin{array}{llll}0.3990783820041571 & 0.4447808578806413 & 0.2249671302388251\end{array}$

\section{Pt(111) Surface - vdW-DF-optB88:}

$\mathrm{Pt}$

1.00000000000000

$\begin{array}{lll}14.0790000000000006 & 0.0000000000000000 & 0.0000000000000000\end{array}$

$\begin{array}{lll}0.0000000000000000 & 14.6310000000000002 & 0.0000000000000000\end{array}$

$\begin{array}{llll}0.0000000000000000 & 0.0000000000000000 & 35.0000000000000000\end{array}$

$\mathrm{Pt}$

90

Direct

$\begin{array}{llll}0.7005809324617136 & 0.7228770796061964 & 0.1318717097246009\end{array}$

$\begin{array}{llll}0.9006695276236349 & 0.3895719168890974 & 0.1318117651532733\end{array}$

$\begin{array}{llll}0.9006431532248944 & 0.7228509274240470 & 0.1318392144110211\end{array}$

$\begin{array}{llll}0.8009310445569606 & 0.5562066536873164 & 0.1320268209003012\end{array}$

$\begin{array}{llll}0.3005830801316699 & 0.7227852504673802 & 0.1318179583626374\end{array}$

$\begin{array}{llll}0.5006489407806605 & 0.3895832726601672 & 0.1318147343487174\end{array}$

$\begin{array}{llll}0.5006056834405990 & 0.7227567832386920 & 0.1318058408776486\end{array}$

$\begin{array}{llll}0.6007089445619024 & 0.5562144554348649 & 0.1319775053922530\end{array}$

$\begin{array}{llll}0.4008420336439314 & 0.5562468415421109 & 0.1322704357485346\end{array}$

$\begin{array}{llll}0.7005537915086770 & 0.0560831896210170 & 0.1320157320939649\end{array}$

$\begin{array}{llll}0.7006621241425135 & 0.3895733406285902 & 0.1318148298918426\end{array}$

$\begin{array}{llll}0.8005694238328470 & 0.2226527437612491 & 0.1317170661398725\end{array}$

$\begin{array}{llll}0.6005764209398453 & 0.2226978225081966 & 0.1317522211539123\end{array}$

$\begin{array}{lllll}0.9010126251996770 & 0.0561782563111176 & 0.1320426940729990\end{array}$

$\begin{array}{lllll}0.8005461453240313 & 0.8895426084099131 & 0.1319665508986881\end{array}$

$\begin{array}{llll}0.1006808445262089 & 0.3895799844160740 & 0.1318188410640689\end{array}$

$\begin{array}{llll}0.1005914594432371 & 0.7229033866299710 & 0.1320538950220483\end{array}$

$\begin{array}{llll}0.2010464147418343 & 0.5562382643166766 & 0.1320825966787633\end{array}$

$\begin{array}{llll}0.0011307602023506 & 0.5562375337326158 & 0.1321234408161319\end{array}$

$\begin{array}{llll}0.3006964116917549 & 0.0561789613014683 & 0.1320340840454282\end{array}$

$\begin{array}{llll}0.3006359034798525 & 0.3895886412472079 & 0.1318135960038073\end{array}$

$\begin{array}{llll}0.4006589146333258 & 0.2228762926398404 & 0.1318319101152887\end{array}$

$\begin{array}{llll}0.2006071984243976 & 0.2228572985309779 & 0.1318615953323830\end{array}$

$\begin{array}{llll}0.5004579714927945 & 0.0560768667490028 & 0.1320828556275979\end{array}$

$\begin{array}{llll}0.6004552654662647 & 0.8895175231686852 & 0.1319436308630680\end{array}$

$\begin{array}{llll}0.4004779833160355 & 0.8895082789071046 & 0.1319414006113843\end{array}$

$\begin{array}{llll}0.0006336032962569 & 0.2227649480196693 & 0.1317979137297608\end{array}$

$\begin{array}{lllll}0.1011408788352658 & 0.0561825325587941 & 0.1321114247731268\end{array}$

$\begin{array}{llll}0.2008479865077612 & 0.8894768222569108 & 0.1322158186439459\end{array}$

$\begin{array}{llll}0.0007084813676215 & 0.8895365533298559 & 0.1318323530937272\end{array}$ 
$\begin{array}{llll}0.9000160000000008 & 0.6111420000000010 & 0.0656899999999965\end{array}$

$\begin{array}{llll}0.8000200000000035 & 0.7778080000000003 & 0.0656899999999965\end{array}$

$\begin{array}{llll}0.5000110000000006 & 0.6111420000000010 & 0.0656899999999965\end{array}$

$\begin{array}{llll}0.6000170000000011 & 0.7778080000000003 & 0.0656899999999965\end{array}$

$\begin{array}{llll}0.4000050000000002 & 0.7778080000000003 & 0.0656899999999965\end{array}$

$\begin{array}{llll}0.7000129999999984 & 0.2777880000000010 & 0.0656899999999965\end{array}$

$\begin{array}{llll}0.7000129999999984 & 0.6111420000000010 & 0.0656899999999965\end{array}$

$\begin{array}{llll}0.8000200000000035 & 0.4444639999999964 & 0.0656899999999965\end{array}$

$\begin{array}{llll}0.6000170000000011 & 0.4444639999999964 & 0.0656899999999965\end{array}$

$\begin{array}{llll}0.9000160000000008 & 0.9444459999999992 & 0.0656899999999965\end{array}$

$\begin{array}{lllll}0.9000160000000008 & 0.2777880000000010 & 0.0656899999999965\end{array}$

$\begin{array}{lllll}0.8000200000000035 & 0.1111220000000017 & 0.0656899999999965\end{array}$

$\begin{array}{llll}0.1000060000000005 & 0.6111420000000010 & 0.0656899999999965\end{array}$

$\begin{array}{llll}0.2000019999999978 & 0.7778080000000003 & 0.0656899999999965\end{array}$

$\begin{array}{llll}0.0000000000000000 & 0.7778080000000003 & 0.0656899999999965\end{array}$

$\begin{array}{llll}0.3000090000000029 & 0.2777880000000010 & 0.0656899999999965\end{array}$

$\begin{array}{llll}0.3000090000000029 & 0.6111420000000010 & 0.0656899999999965\end{array}$

$\begin{array}{llll}0.4000050000000002 & 0.4444639999999964 & 0.0656899999999965\end{array}$

$\begin{array}{llll}0.2000019999999978 & 0.4444639999999964 & 0.0656899999999965\end{array}$

$\begin{array}{llll}0.5000110000000006 & 0.9444459999999992 & 0.0656899999999965\end{array}$

$\begin{array}{lllll}0.5000110000000006 & 0.2777880000000010 & 0.0656899999999965\end{array}$

$\begin{array}{llll}0.6000170000000011 & 0.1111220000000017 & 0.0656899999999965\end{array}$

$\begin{array}{lllll}0.4000050000000002 & 0.1111220000000017 & 0.0656899999999965\end{array}$

$\begin{array}{llll}0.7000129999999984 & 0.9444459999999992 & 0.0656899999999965\end{array}$

$\begin{array}{llll}0.0000000000000000 & 0.4444639999999964 & 0.0656899999999965\end{array}$

$\begin{array}{lllll}0.1000060000000005 & 0.9444459999999992 & 0.0656899999999965\end{array}$

$\begin{array}{lllll}0.1000060000000005 & 0.2777880000000010 & 0.0656899999999965\end{array}$

$\begin{array}{llll}0.2000019999999978 & 0.1111220000000017 & 0.0656899999999965\end{array}$

$\begin{array}{lllll}0.0000000000000000 & 0.1111220000000017 & 0.0656899999999965\end{array}$

$\begin{array}{llll}0.3000090000000029 & 0.9444459999999992 & 0.0656899999999965\end{array}$

$\begin{array}{llll}0.9000160000000008 & 0.8333640000000031 & 0.0000000000000000\end{array}$

$\begin{array}{lllll}0.5000110000000006 & 0.8333640000000031 & 0.0000000000000000\end{array}$

$\begin{array}{llll}0.7000129999999984 & 0.5000199999999992 & 0.0000000000000000\end{array}$

$\begin{array}{llll}0.7000129999999984 & 0.8333640000000031 & 0.0000000000000000\end{array}$

$\begin{array}{lllll}0.8000200000000035 & 0.6666979999999967 & 0.0000000000000000\end{array}$

$\begin{array}{lllll}0.6000069999999980 & 0.6666979999999967 & 0.0000000000000000\end{array}$

$\begin{array}{llll}0.9000160000000008 & 0.1666760000000025 & 0.0000000000000000\end{array}$

$\begin{array}{llll}0.9000160000000008 & 0.5000199999999992 & 0.0000000000000000\end{array}$

$\begin{array}{llll}0.8000200000000035 & 0.3333439999999968 & 0.0000000000000000\end{array}$

$\begin{array}{lllll}0.1000060000000005 & 0.8333640000000031 & 0.0000000000000000\end{array}$

$\begin{array}{llll}0.3000090000000029 & 0.5000199999999992 & 0.0000000000000000\end{array}$

$\begin{array}{llllll}0.3000090000000029 & 0.8333640000000031 & 0.0000000000000000\end{array}$

$\begin{array}{llll}0.4000050000000002 & 0.6666979999999967 & 0.0000000000000000\end{array}$

$\begin{array}{llll}0.2000019999999978 & 0.6666979999999967 & 0.0000000000000000\end{array}$

$\begin{array}{llll}0.5000110000000006 & 0.1666760000000025 & 0.0000000000000000\end{array}$

$\begin{array}{llll}0.5000110000000006 & 0.5000199999999992 & 0.0000000000000000\end{array}$

$\begin{array}{llll}0.6000069999999980 & 0.3333439999999968 & 0.0000000000000000\end{array}$ 
$\begin{array}{llll}0.4000050000000002 & 0.3333439999999968 & 0.0000000000000000\end{array}$

$\begin{array}{lll}0.7000129999999984 & 0.1666760000000025 & 0.0000000000000000\end{array}$

$\begin{array}{llll}0.8000200000000035 & 0.0000000000000000 & 0.0000000000000000\end{array}$

$\begin{array}{llll}0.6000069999999980 & 0.0000000000000000 & 0.0000000000000000\end{array}$

$\begin{array}{llll}0.0000000000000000 & 0.6666979999999967 & 0.0000000000000000\end{array}$

$0.1000060000000005 \quad 0.16667600000000250 .0000000000000000$

$\begin{array}{llll}0.1000060000000005 & 0.5000199999999992 & 0.0000000000000000\end{array}$

$\begin{array}{llll}0.2000019999999978 & 0.3333439999999968 & 0.0000000000000000\end{array}$

$\begin{array}{llll}0.0000000000000000 & 0.3333439999999968 & 0.0000000000000000\end{array}$

$\begin{array}{llll}0.3000090000000029 & 0.1666760000000025 & 0.0000000000000000\end{array}$

$\begin{array}{lll}0.4000050000000002 & 0.0000000000000000 & 0.0000000000000000\end{array}$

$\begin{array}{llll}0.2000019999999978 & 0.0000000000000000 & 0.0000000000000000\end{array}$

$\begin{array}{llll}0.0000000000000000 & 0.0000000000000000 & 0.0000000000000000\end{array}$

\section{FeP - vdW-DF-revPBE, Ueff $=3$;}

$\mathrm{H} \mathrm{C} \mathrm{N} \mathrm{Fe}$

1.00000000000000

$\begin{array}{llll}14.2799999999999994 & 0.0000000000000000 & 0.0000000000000000\end{array}$

$\begin{array}{llll}0.0000000000000000 & 14.8409999999999993 & 0.0000000000000000\end{array}$

$\begin{array}{llll}0.0000000000000000 & 0.0000000000000000 & 35.0000000000000000\end{array}$

$\mathrm{H} \quad \mathrm{C} \quad \mathrm{N} \quad \mathrm{Fe}$

$\begin{array}{llll}12 & 20 & 4 & 1\end{array}$

Direct

$\begin{array}{llll}0.7164292494728403 & 0.4460067730004078 & 0.2267437177920575\end{array}$

$\begin{array}{llll}0.4001513493699028 & 0.1390633299396206 & 0.2271578487818184\end{array}$

$\begin{array}{llll}0.0814811788261632 & 0.4438823201596734 & 0.2270945523707927\end{array}$

$\begin{array}{llll}0.3978591114548473 & 0.7507063217788814 & 0.2270103215473351\end{array}$

$\begin{array}{llll}0.0787944970618984 & 0.6217346466477132 & 0.2280010012593566\end{array}$

$\begin{array}{llll}0.2122480146909854 & 0.7515651058952860 & 0.2279657908061287\end{array}$

$\begin{array}{llll}0.5833059782949764 & 0.7532634587618275 & 0.2278629825485969\end{array}$

$\begin{array}{lllll}0.7179787152207441 & 0.6243187116358995 & 0.2277724564208433\end{array}$

$\begin{array}{llll}0.7192717042987381 & 0.2672716894850680 & 0.2275698928370085\end{array}$

$\begin{array}{llll}0.5857404258668437 & 0.1377358599462888 & 0.2279091153311143\end{array}$

$\begin{array}{llll}0.2146179671531071 & 0.1365423429870931 & 0.2282382149459542\end{array}$

$\begin{array}{lllll}0.0800332984254179 & 0.2651973899453751 & 0.2282179528918847\end{array}$

$\begin{array}{lllll}0.3115711037643357 & 0.6337969763294934 & 0.2263035492212177\end{array}$

$\begin{array}{llll}0.2216774875929903 & 0.6791236274331823 & 0.2272461130145849\end{array}$

$\begin{array}{llll}0.1542299582689318 & 0.6135840825315797 & 0.2272347306321585\end{array}$

$\begin{array}{llll}0.2023507145058475 & 0.5278163126853744 & 0.2263270466230116\end{array}$

$\begin{array}{llll}0.3982389496164495 & 0.6774492889017437 & 0.2264062575930401\end{array}$

$\begin{array}{lllll}0.4852605386611105 & 0.6346402491341181 & 0.2262562696239497\end{array}$

$\begin{array}{llll}0.5951741473582947 & 0.5293142581846261 & 0.2262845659335060\end{array}$

$\begin{array}{llll}0.6426485206955306 & 0.6155913493327900 & 0.2271495118293524\end{array}$

$\begin{array}{llll}0.5746760902296930 & 0.6807363888800738 & 0.2271419700780104\end{array}$

$\begin{array}{lllll}0.6402201542409769 & 0.4457764279815027 & 0.2262621162263008\end{array}$

$\begin{array}{lllll}0.5958836938567923 & 0.3618101106710017 & 0.2261212299832072\end{array}$

$\begin{array}{llll}0.4866427567897252 & 0.2557023387507566 & 0.2263709914811517\end{array}$ 
$\begin{array}{llll}0.5763177584191406 & 0.2101931880998347 & 0.2271676731444255\end{array}$

$\begin{array}{llll}0.6438787058948223 & 0.2757509704116061 & 0.2269507215054466\end{array}$

$\begin{array}{llll}0.3998399398610855 & 0.2123208475130784 & 0.2265908841340698\end{array}$

$\begin{array}{llll}0.3127256239845762 & 0.2551100404198180 & 0.2265156181129190\end{array}$

$\begin{array}{llll}0.2026880745277392 & 0.3605256859361477 & 0.2265143018028924\end{array}$

$\begin{array}{llll}0.1576622836100312 & 0.4441080674626680 & 0.2265575333046597\end{array}$

$\begin{array}{llll}0.1553171175354373 & 0.2741503774960404 & 0.2274420440863485\end{array}$

$\begin{array}{llll}0.2233194810833573 & 0.2090430477019541 & 0.2273622224574496\end{array}$

$\begin{array}{llll}0.2985851052826072 & 0.5410103961210654 & 0.2257216103561784\end{array}$

$\begin{array}{llll}0.4988512689636911 & 0.5419081773581002 & 0.2257463454028004\end{array}$

$\begin{array}{lllll}0.2990599867540731 & 0.3478467623308804 & 0.2259952632418261\end{array}$

$\begin{array}{lllll}0.4996559719304536 & 0.3484945133429721 & 0.2257920142568077\end{array}$

$\begin{array}{llll}0.3990829242589802 & 0.4447753205101398 & 0.2255409751896341\end{array}$

\section{Pt(111) Surface - vdW-DF-revPBE:}

$\mathrm{Pt}$

1.00000000000000

$\begin{array}{lll}14.2799999999999994 & 0.0000000000000000 & 0.0000000000000000\end{array}$

$\begin{array}{lll}0.0000000000000000 & 14.8409999999999993 & 0.0000000000000000\end{array}$

$\begin{array}{llll}0.0000000000000000 & 0.0000000000000000 & 35.0000000000000000\end{array}$

$\mathrm{Pt}$

90

Direct

$\begin{array}{llll}0.7999999999999972 & 0.6666669999999968 & 0.0000000000000000\end{array}$

$\begin{array}{lllll}0.9000040000000027 & 0.8333319999999986 & 0.0000000000000000\end{array}$

$\begin{array}{lllll}0.7999999999999972 & 0.3333330000000032 & 0.0000000000000000\end{array}$

$\begin{array}{llll}0.9000040000000027 & 0.4999990000000025 & 0.0000000000000000\end{array}$

$\begin{array}{llll}0.7999999999999972 & 0.0000000000000000 & 0.0000000000000000\end{array}$

$\begin{array}{llll}0.9000040000000027 & 0.1666650000000018 & 0.0000000000000000\end{array}$

$\begin{array}{llll}0.6000090000000000 & 0.6666669999999968 & 0.0000000000000000\end{array}$

$\begin{array}{lllll}0.7000140000000030 & 0.8333319999999986 & 0.0000000000000000\end{array}$

$\begin{array}{lllll}0.3999999999999986 & 0.6666669999999968 & 0.0000000000000000\end{array}$

$\begin{array}{llll}0.5000039999999970 & 0.8333319999999986 & 0.0000000000000000\end{array}$

$\begin{array}{llll}0.6000090000000000 & 0.3333330000000032 & 0.0000000000000000\end{array}$

$\begin{array}{lllll}0.7000140000000030 & 0.4999990000000025 & 0.0000000000000000\end{array}$

$\begin{array}{llll}0.3999999999999986 & 0.3333330000000032 & 0.0000000000000000\end{array}$

$\begin{array}{llll}0.5000039999999970 & 0.4999990000000025 & 0.0000000000000000\end{array}$

$\begin{array}{lll}0.6000090000000000 & 0.0000000000000000 & 0.0000000000000000\end{array}$

$\begin{array}{llll}0.7000140000000030 & 0.1666650000000018 & 0.0000000000000000\end{array}$

$\begin{array}{lllll}0.3999999999999986 & 0.0000000000000000 & 0.0000000000000000\end{array}$

$\begin{array}{llll}0.5000039999999970 & 0.1666650000000018 & 0.0000000000000000\end{array}$

$\begin{array}{llll}0.2000090000000014 & 0.6666669999999968 & 0.0000000000000000\end{array}$

$\begin{array}{llll}0.3000139999999973 & 0.8333319999999986 & 0.0000000000000000\end{array}$

$\begin{array}{llll}0.0000000000000000 & 0.6666669999999968 & 0.0000000000000000\end{array}$

$\begin{array}{llll}0.1000039999999984 & 0.8333319999999986 & 0.0000000000000000\end{array}$

$\begin{array}{lllll}0.2000090000000014 & 0.3333330000000032 & 0.0000000000000000\end{array}$

$\begin{array}{llll}0.3000139999999973 & 0.4999990000000025 & 0.0000000000000000\end{array}$ 
$\begin{array}{llll}0.0000000000000000 & 0.3333330000000032 & 0.0000000000000000\end{array}$

$\begin{array}{llll}0.1000039999999984 & 0.4999990000000025 & 0.0000000000000000\end{array}$

$\begin{array}{llll}0.2000090000000014 & 0.0000000000000000 & 0.0000000000000000\end{array}$

$\begin{array}{llll}0.3000139999999973 & 0.1666650000000018 & 0.0000000000000000\end{array}$

$\begin{array}{llll}0.0000000000000000 & 0.0000000000000000 & 0.0000000000000000\end{array}$

0.10000399999999840 .16666500000000180 .0000000000000000

$\begin{array}{lllll}0.9000040000000027 & 0.7222220000000021 & 0.0666289999999989\end{array}$

$\begin{array}{llll}0.7999999999999972 & 0.8888869999999969 & 0.0666289999999989\end{array}$

$\begin{array}{lllll}0.9000040000000027 & 0.3888880000000015 & 0.0666289999999989\end{array}$

$\begin{array}{llll}0.7999999999999972 & 0.5555540000000008 & 0.0666289999999989\end{array}$

$\begin{array}{llll}0.9000040000000027 & 0.0555549999999982 & 0.0666289999999989\end{array}$

$\begin{array}{lllll}0.7999999999999972 & 0.2222209999999976 & 0.0666289999999989\end{array}$

$\begin{array}{llll}0.6000090000000000 & 0.8888869999999969 & 0.0666289999999989\end{array}$

$\begin{array}{llll}0.7000140000000030 & 0.7222220000000021 & 0.0666289999999989\end{array}$

$\begin{array}{lllll}0.5000039999999970 & 0.7222220000000021 & 0.0666289999999989\end{array}$

$\begin{array}{llll}0.3999999999999986 & 0.8888869999999969 & 0.0666289999999989\end{array}$

$\begin{array}{llll}0.6000090000000000 & 0.5555540000000008 & 0.0666289999999989\end{array}$

$\begin{array}{llll}0.7000140000000030 & 0.3888880000000015 & 0.0666289999999989\end{array}$

$\begin{array}{llll}0.5000039999999970 & 0.3888880000000015 & 0.0666289999999989\end{array}$

$\begin{array}{llll}0.3999999999999986 & 0.5555540000000008 & 0.0666289999999989\end{array}$

$\begin{array}{lllll}0.6000090000000000 & 0.2222209999999976 & 0.0666289999999989\end{array}$

$\begin{array}{llll}0.7000140000000030 & 0.0555549999999982 & 0.0666289999999989\end{array}$

$\begin{array}{llll}0.5000039999999970 & 0.0555549999999982 & 0.0666289999999989\end{array}$

$\begin{array}{lllll}0.3999999999999986 & 0.2222209999999976 & 0.0666289999999989\end{array}$

$\begin{array}{llll}0.2000090000000014 & 0.8888869999999969 & 0.0666289999999989\end{array}$

$\begin{array}{lllll}0.3000139999999973 & 0.7222220000000021 & 0.0666289999999989\end{array}$

$\begin{array}{lllll}0.1000039999999984 & 0.7222220000000021 & 0.0666289999999989\end{array}$

$\begin{array}{llll}0.0000000000000000 & 0.8888869999999969 & 0.0666289999999989\end{array}$

$\begin{array}{llll}0.2000090000000014 & 0.5555540000000008 & 0.0666289999999989\end{array}$

$\begin{array}{llll}0.3000139999999973 & 0.3888880000000015 & 0.0666289999999989\end{array}$

$\begin{array}{llll}0.1000039999999984 & 0.3888880000000015 & 0.0666289999999989\end{array}$

$\begin{array}{llll}0.0000000000000000 & 0.5555540000000008 & 0.0666289999999989\end{array}$

$\begin{array}{lllll}0.2000090000000014 & 0.2222209999999976 & 0.0666289999999989\end{array}$

$\begin{array}{llll}0.3000139999999973 & 0.0555549999999982 & 0.0666289999999989\end{array}$

$\begin{array}{lllll}0.1000039999999984 & 0.0555549999999982 & 0.0666289999999989\end{array}$

$\begin{array}{lllll}0.0000000000000000 & 0.2222209999999976 & 0.0666289999999989\end{array}$

$\begin{array}{llll}0.8000423827989948 & 0.7778995164634616 & 0.1339503658347707\end{array}$

$\begin{array}{llll}0.9000467977322117 & 0.9445752739977890 & 0.1339508449099222\end{array}$

$\begin{array}{llll}0.8000423827989948 & 0.4445665164634583 & 0.1339503658347707\end{array}$

$\begin{array}{lllll}0.9000467977322117 & 0.6112422739977857 & 0.1339508449099222\end{array}$

$\begin{array}{lllll}0.8000423827989948 & 0.1112325164634578 & 0.1339503658347707\end{array}$

$\begin{array}{llll}0.9000467977322117 & 0.2779092739977894 & 0.1339508449099222\end{array}$

$\begin{array}{llll}0.4000497577772301 & 0.7778980765194031 & 0.1339323124438436\end{array}$

$\begin{array}{llll}0.6000378612689158 & 0.7778948403770621 & 0.1339403979229549\end{array}$

$\begin{array}{llll}0.7000378605815294 & 0.9445615844589109 & 0.1339454418308394\end{array}$

$\begin{array}{llll}0.5000326060900170 & 0.9445749846652679 & 0.1339344758456162\end{array}$

$\begin{array}{llll}0.4000497577772301 & 0.4445650765193999 & 0.1339323124438436\end{array}$ 
$\begin{array}{llll}0.6000378612689158 & 0.4445618403770589 & 0.1339403979229549\end{array}$

$\begin{array}{lllll}0.7000378605815294 & 0.6112285844589077 & 0.1339454418308394\end{array}$

$\begin{array}{lllll}0.5000326060900170 & 0.6112419846652647 & 0.1339344758456162\end{array}$

$\begin{array}{llll}0.4000497577772301 & 0.1112310765193994 & 0.1339323124438436\end{array}$

$\begin{array}{llll}0.6000378612689158 & 0.1112278403770582 & 0.1339403979229549\end{array}$

$\begin{array}{llll}0.7000378605815294 & 0.2778955844589116 & 0.1339454418308394\end{array}$

$\begin{array}{llll}0.5000326060900170 & 0.2779089846652684 & 0.1339344758456162\end{array}$

$\begin{array}{lllll}0.0000540265184684 & 0.7779089316908264 & 0.1339513406538936\end{array}$

$\begin{array}{llll}0.2000712580240303 & 0.7778975579757280 & 0.1339409895885965\end{array}$

$\begin{array}{llll}0.3000548419146843 & 0.9445543802947091 & 0.1339332610210253\end{array}$

$\begin{array}{llll}0.1000604036616866 & 0.9445725219977632 & 0.1339481058655297\end{array}$

$\begin{array}{lllll}0.0000540265184684 & 0.4445759316908231 & 0.1339513406538936\end{array}$

$\begin{array}{lllll}0.2000712580240303 & 0.4445645579757251 & 0.1339409895885965\end{array}$

$\begin{array}{llll}0.3000548419146843 & 0.6112213802947059 & 0.1339332610210253\end{array}$

$\begin{array}{llll}0.1000604036616866 & 0.6112395219977600 & 0.1339481058655297\end{array}$

$\begin{array}{llll}0.0000540265184684 & 0.1112419316908223 & 0.1339513406538936\end{array}$

$\begin{array}{llll}0.2000712580240303 & 0.1112305579757244 & 0.1339409895885965\end{array}$

$\begin{array}{llll}0.3000548419146843 & 0.2778883802947101 & 0.1339332610210253\end{array}$

$\begin{array}{llll}0.1000604036616866 & 0.2779065219977642 & 0.1339481058655297\end{array}$ 
Section S11: Coordinates of FeP on the Pt(111) Surface (POSCAR format)

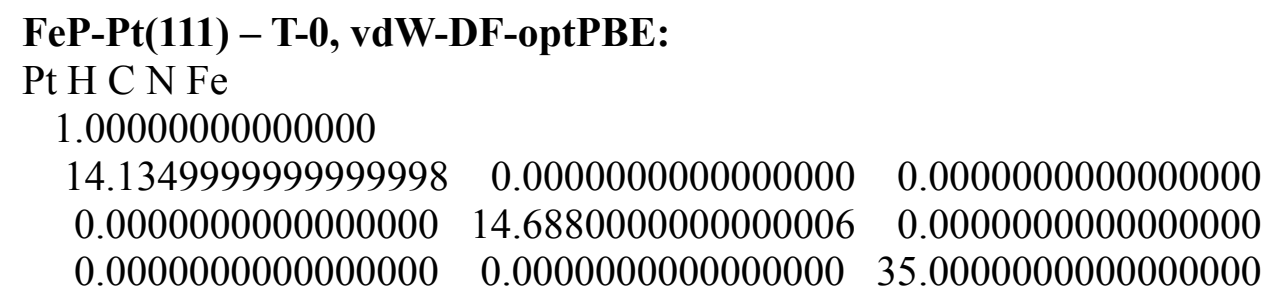

$\begin{array}{lllll}\mathrm{Pt} & \mathrm{H} & \mathrm{C} & \mathrm{N} & \mathrm{Fe}\end{array}$

$\begin{array}{lllll}90 & 12 & 20 & 4 & 1\end{array}$

Direct

0.69989999999999950 .83332999999999660 .0000000000000000

$\begin{array}{llll}0.7998899999999978 & 0.6666700000000034 & 0.0000000000000000\end{array}$

$\begin{array}{lll}0.5999199999999973 & 0.6666700000000034 & 0.0000000000000000\end{array}$

$\begin{array}{llll}0.8998800000000031 & 0.8333299999999966 & 0.0000000000000000\end{array}$

$\begin{array}{llll}0.2999599999999987 & 0.8333299999999966 & 0.0000000000000000\end{array}$

$\begin{array}{llll}0.3999499999999969 & 0.6666700000000034 & 0.0000000000000000\end{array}$

$0.1999700000000004 \quad 0.66667000000000340 .0000000000000000$

$\begin{array}{llll}0.4999299999999991 & 0.8333299999999966 & 0.0000000000000000\end{array}$

$\begin{array}{llll}0.0000000000000000 & 0.6666700000000034 & 0.0000000000000000\end{array}$

$\begin{array}{lllll}0.0999899999999982 & 0.8333299999999966 & 0.0000000000000000\end{array}$

$\begin{array}{lllll}0.6998999999999995 & 0.1666600000000003 & 0.0000000000000000\end{array}$

$\begin{array}{llll}0.7998899999999978 & 0.0000000000000000 & 0.0000000000000000\end{array}$

$\begin{array}{llll}0.5999199999999973 & 0.0000000000000000 & 0.0000000000000000\end{array}$

$\begin{array}{llll}0.8998800000000031 & 0.4999800000000008 & 0.0000000000000000\end{array}$

$\begin{array}{lllll}0.8998800000000031 & 0.1666600000000003 & 0.0000000000000000\end{array}$

$\begin{array}{llll}0.7998899999999978 & 0.3333200000000005 & 0.0000000000000000\end{array}$

$\begin{array}{llll}0.2999599999999987 & 0.1666600000000003 & 0.0000000000000000\end{array}$

$\begin{array}{llll}0.3999499999999969 & 0.0000000000000000 & 0.0000000000000000\end{array}$

$\begin{array}{lll}0.1999700000000004 & 0.0000000000000000 & 0.0000000000000000\end{array}$

$\begin{array}{llll}0.4999299999999991 & 0.4999800000000008 & 0.0000000000000000\end{array}$

$\begin{array}{llll}0.4999299999999991 & 0.1666600000000003 & 0.0000000000000000\end{array}$

$\begin{array}{llll}0.5999199999999973 & 0.3333200000000005 & 0.0000000000000000\end{array}$

$\begin{array}{llll}0.3999499999999969 & 0.3333200000000005 & 0.0000000000000000\end{array}$

$\begin{array}{lll}0.6998999999999995 & 0.4999800000000008 & 0.0000000000000000\end{array}$

$\begin{array}{lllll}0.0000000000000000 & 0.0000000000000000 & 0.0000000000000000\end{array}$

$\begin{array}{llll}0.0999899999999982 & 0.4999800000000008 & 0.0000000000000000\end{array}$

$\begin{array}{llll}0.0999899999999982 & 0.1666600000000003 & 0.0000000000000000\end{array}$

$\begin{array}{llll}0.1999700000000004 & 0.3333200000000005 & 0.0000000000000000\end{array}$

$\begin{array}{llll}0.0000000000000000 & 0.3333200000000005 & 0.0000000000000000\end{array}$

$\begin{array}{llll}0.2999599999999987 & 0.4999800000000008 & 0.0000000000000000\end{array}$

$\begin{array}{llll}0.8998800000000031 & 0.7222200000000001 & 0.0659399999999977\end{array}$

$\begin{array}{lllll}0.4999299999999991 & 0.7222200000000001 & 0.0659399999999977\end{array}$

$\begin{array}{lllll}0.6998999999999995 & 0.7222200000000001 & 0.0659399999999977\end{array}$

$\begin{array}{lllll}0.7998899999999978 & 0.8888800000000003 & 0.0659399999999977\end{array}$

$\begin{array}{lllll}0.5999199999999973 & 0.8888800000000003 & 0.0659399999999977\end{array}$

$\begin{array}{lllll}0.0999899999999982 & 0.7222200000000001 & 0.0659399999999977\end{array}$ 
$\begin{array}{llll}0.2999599999999987 & 0.7222200000000001 & 0.0659399999999977\end{array}$

$\begin{array}{llll}0.3999499999999969 & 0.8888800000000003 & 0.0659399999999977\end{array}$

$\begin{array}{lllll}0.1999700000000004 & 0.8888800000000003 & 0.0659399999999977\end{array}$

$\begin{array}{llll}0.0000000000000000 & 0.8888800000000003 & 0.0659399999999977\end{array}$

$\begin{array}{lllll}0.8998800000000031 & 0.0555499999999967 & 0.0659399999999977\end{array}$

$\begin{array}{lllll}0.4999299999999991 & 0.0555499999999967 & 0.0659399999999977\end{array}$

$\begin{array}{lllll}0.6998999999999995 & 0.3888800000000003 & 0.0659399999999977\end{array}$

$\begin{array}{llllll}0.6998999999999995 & 0.0555499999999967 & 0.0659399999999977\end{array}$

$\begin{array}{lllll}0.7998899999999978 & 0.2222099999999969 & 0.0659399999999977\end{array}$

$\begin{array}{llll}0.5999199999999973 & 0.2222099999999969 & 0.0659399999999977\end{array}$

$\begin{array}{llll}0.8998800000000031 & 0.3888800000000003 & 0.0659399999999977\end{array}$

$\begin{array}{lllll}0.7998899999999978 & 0.5555400000000006 & 0.0659399999999977\end{array}$

$\begin{array}{lllll}0.0999899999999982 & 0.0555499999999967 & 0.0659399999999977\end{array}$

$\begin{array}{lllll}0.2999599999999987 & 0.3888800000000003 & 0.0659399999999977\end{array}$

$\begin{array}{lllll}0.2999599999999987 & 0.0555499999999967 & 0.0659399999999977\end{array}$

$\begin{array}{lllll}0.3999499999999969 & 0.2222099999999969 & 0.0659399999999977\end{array}$

$\begin{array}{lllll}0.1999700000000004 & 0.2222099999999969 & 0.0659399999999977\end{array}$

$\begin{array}{lllll}0.4999299999999991 & 0.3888800000000003 & 0.0659399999999977\end{array}$

$\begin{array}{llll}0.5999199999999973 & 0.5555400000000006 & 0.0659399999999977\end{array}$

$\begin{array}{llll}0.3999499999999969 & 0.5555400000000006 & 0.0659399999999977\end{array}$

$\begin{array}{llllll}0.0000000000000000 & 0.2222099999999969 & 0.0659399999999977\end{array}$

$\begin{array}{lllll}0.0999899999999982 & 0.3888800000000003 & 0.0659399999999977\end{array}$

$\begin{array}{lllll}0.1999700000000004 & 0.5555400000000006 & 0.0659399999999977\end{array}$

$\begin{array}{llll}0.0000000000000000 & 0.5555400000000006 & 0.0659399999999977\end{array}$

$\begin{array}{llll}0.9008913826279462 & 0.9423539257857284 & 0.1326980101743489\end{array}$

$\begin{array}{llll}0.8006429701211093 & 0.7767086140936322 & 0.1322355642576367\end{array}$

$\begin{array}{llll}0.5003717270213042 & 0.9377480458663290 & 0.1326625251600291\end{array}$

$\begin{array}{llll}0.6004002699626836 & 0.7721652297834565 & 0.1390371722167353\end{array}$

$\begin{array}{llll}0.3981479690976990 & 0.7750391401133261 & 0.1377198066908766\end{array}$

$\begin{array}{llll}0.6997380783802515 & 0.9398751463338111 & 0.1324412913732004\end{array}$

$\begin{array}{lllll}0.1020426413776304 & 0.9438148998207927 & 0.1322492257784636\end{array}$

$\begin{array}{llll}0.1979161795422713 & 0.7722787214215282 & 0.1390602659233326\end{array}$

$\begin{array}{llll}0.9991317024639476 & 0.7774785307299209 & 0.1322725923627487\end{array}$

$\begin{array}{llll}0.2984974794438269 & 0.9413803313554067 & 0.1326162820434653\end{array}$

$\begin{array}{llll}0.8980658613902270 & 0.2717671013605618 & 0.1324896818488409\end{array}$

$\begin{array}{lllll}0.7997008932280854 & 0.1066844090732514 & 0.1323725902356637\end{array}$

$\begin{array}{llll}0.5004145633698751 & 0.2706950726422259 & 0.1293610885151730\end{array}$

$\begin{array}{llll}0.5993632371267047 & 0.1041319783023001 & 0.1332206873788251\end{array}$

$\begin{array}{llll}0.4007769622287335 & 0.1024114223365182 & 0.1321141590429083\end{array}$

$\begin{array}{lllll}0.7016605128017634 & 0.6104106078097169 & 0.1353336582282151\end{array}$

$\begin{array}{lllll}0.6968990634348492 & 0.2711502153196590 & 0.1389660618120612\end{array}$

$\begin{array}{llll}0.8025992792800378 & 0.4406499584601067 & 0.1310804731153903\end{array}$

$\begin{array}{llll}0.6067427250919866 & 0.4412695864753827 & 0.1346296257402733\end{array}$

$\begin{array}{llll}0.8990851627597706 & 0.6098080082577981 & 0.1324865974411960\end{array}$

$\begin{array}{lllll}0.0996375527954383 & 0.2727388005010484 & 0.1364467925048634\end{array}$

$\begin{array}{lllll}0.2053098389149451 & 0.1143605059257036 & 0.1429957373204573\end{array}$

$\begin{array}{llll}0.0014301554698802 & 0.1074326108139115 & 0.1321864280907791\end{array}$ 
$\begin{array}{llll}0.3017938374887924 & 0.6109752747992747 & 0.1295919640308468\end{array}$ $\begin{array}{lllll}0.3028662882687168 & 0.2735701350997291 & 0.1290587569506428\end{array}$ $\begin{array}{llll}0.4005914451284041 & 0.4408888821431027 & 0.1303348418159241\end{array}$ $\begin{array}{llll}0.1916477263064884 & 0.4414630739116689 & 0.1340761235513248\end{array}$ $\begin{array}{llll}0.4964642452382559 & 0.6118509815059813 & 0.1296330728572974\end{array}$ $\begin{array}{llll}0.9947392603327008 & 0.4411667247652585 & 0.1309298599943834\end{array}$ $\begin{array}{lllll}0.0962865237937537 & 0.6101804783352797 & 0.1348375642262073\end{array}$ $\begin{array}{llll}0.7143417660720973 & 0.4490586673818579 & 0.1998776517987502\end{array}$ $\begin{array}{llll}0.4010553469788631 & 0.1322133380031591 & 0.2091414161199671\end{array}$ $\begin{array}{llll}0.0812556734022036 & 0.4459812677451123 & 0.1990898060891849\end{array}$ $\begin{array}{llll}0.3963625167932953 & 0.7586036113273040 & 0.2113628957251201\end{array}$ $\begin{array}{llll}0.0771935426420977 & 0.6287157129967629 & 0.2098109257021415\end{array}$ $\begin{array}{llll}0.2058967195242986 & 0.7515426085226563 & 0.2128196548472631\end{array}$ $\begin{array}{llll}0.5893258421851907 & 0.7552388179369278 & 0.2130334648483800\end{array}$ $\begin{array}{llll}0.7191494590772275 & 0.6331371196955006 & 0.2102687483251820\end{array}$ $\begin{array}{lllll}0.7195765296973411 & 0.2691475812958019 & 0.2124955813221758\end{array}$ $\begin{array}{lllll}0.5893972642735024 & 0.1342205793629284 & 0.2094615941867267\end{array}$ $\begin{array}{llll}0.2018404073941277 & 0.1367586819347348 & 0.2160066458402091\end{array}$ $\begin{array}{llll}0.0779995708776142 & 0.2615742510588669 & 0.2105276090513148\end{array}$ $\begin{array}{llll}0.3064628603182116 & 0.6462783760305170 & 0.1907452939115064\end{array}$ $\begin{array}{llll}0.2130535494960819 & 0.6961464472306084 & 0.1918151164967907\end{array}$ $\begin{array}{llll}0.1422788975617368 & 0.6195866549435267 & 0.1932592824395457\end{array}$ $\begin{array}{llll}0.1998966939829716 & 0.5360238122240191 & 0.2016877787549197\end{array}$ $\begin{array}{llll}0.3974766413843156 & 0.6987179055139729 & 0.1923835237711888\end{array}$ $\begin{array}{llll}0.4898267634044059 & 0.6489149151203657 & 0.1909596995157396\end{array}$ $\begin{array}{llll}0.5966044542688534 & 0.5399585882169400 & 0.2021477720527316\end{array}$ $\begin{array}{lllll}0.6541452336769203 & 0.6232833602495641 & 0.1937675660268846\end{array}$ $\begin{array}{llll}0.5830882221572438 & 0.6996312638524250 & 0.1920988059126977\end{array}$ $\begin{array}{llll}0.6370286466513365 & 0.4499210389157717 & 0.1984822438519138\end{array}$ $\begin{array}{llll}0.5942415215520206 & 0.3625113190119763 & 0.2055020772657511\end{array}$ $\begin{array}{llll}0.4876570710761662 & 0.2512742610871060 & 0.2107180929870648\end{array}$ $\begin{array}{llll}0.5768696905971039 & 0.2067347654293533 & 0.2057668261007136\end{array}$ $\begin{array}{llll}0.6485910439277583 & 0.2759644063297779 & 0.2003172589854383\end{array}$ $\begin{array}{llll}0.3980375579229458 & 0.2064996230436168 & 0.2103523675149203\end{array}$ $\begin{array}{llll}0.3082137807601638 & 0.2458769309259850 & 0.2053964528496716\end{array}$ $\begin{array}{lllll}0.1998236697787238 & 0.3563285702994481 & 0.2027377221630120\end{array}$ $\begin{array}{lllll}0.1586267810246423 & 0.4458317606421986 & 0.1976095593588822\end{array}$ $\begin{array}{llll}0.1448936038697178 & 0.2704123455933330 & 0.1950745786070972\end{array}$ $\begin{array}{llll}0.2173023125676790 & 0.1955844324357807 & 0.1976962691313834\end{array}$ $\begin{array}{llll}0.2931253868057194 & 0.5542205650309142 & 0.2045814717027746\end{array}$ $\begin{array}{llll}0.5032556275345080 & 0.5573329170339080 & 0.2045026079876302\end{array}$ $\begin{array}{llll}0.2918857277502632 & 0.3400254825237967 & 0.2096526654216447\end{array}$ $\begin{array}{llll}0.5016192801458602 & 0.3468771879358797 & 0.2124250123165464\end{array}$ $\begin{array}{llll}0.3975078561640645 & 0.4487206077329990 & 0.2027390887565068\end{array}$ 
FeP-Pt(111) - T-45, vdW-DF-optPBE:

Pt Fe N C H

1.00000000000000

$\begin{array}{llll}14.1349999999999998 & 0.0000000000000000 & 0.0000000000000000\end{array}$

$\begin{array}{lll}0.0000000000000000 & 14.6880000000000006 & 0.0000000000000000\end{array}$

$\begin{array}{llll}0.0000000000000000 & 0.0000000000000000 & 35.0000000000000000\end{array}$

$\mathrm{Pt} \mathrm{Fe} \mathrm{N} \mathrm{C}$

$\begin{array}{lllll}90 & 1 & 4 & 20 & 12\end{array}$

Direct

$\begin{array}{llll}0.0944442054887356 & 0.6103334469636557 & 0.1327573403945667\end{array}$

$\begin{array}{llll}0.9979202881236245 & 0.4429340304345902 & 0.1330944496324277\end{array}$

$\begin{array}{llll}0.5078530660835908 & 0.6098454382154670 & 0.1332563482841253\end{array}$

$\begin{array}{llll}0.1951764248394378 & 0.4426296381472234 & 0.1300770580659325\end{array}$

$\begin{array}{llll}0.4002957507255522 & 0.4423831854957108 & 0.1310258889720141\end{array}$

$\begin{array}{llll}0.2941819207012291 & 0.2755400251787288 & 0.1330953675089006\end{array}$

$\begin{array}{llll}0.2916961467155295 & 0.6095122113513108 & 0.1330078807178936\end{array}$

$\begin{array}{llll}0.9982736070932094 & 0.1086672462149318 & 0.1328944702386929\end{array}$

$\begin{array}{llll}0.1955078698468438 & 0.1070875825971653 & 0.1311386376748221\end{array}$

$\begin{array}{llll}0.0952168551793357 & 0.2752205952209230 & 0.1326751746614469\end{array}$

$\begin{array}{llll}0.8999457111745670 & 0.6096340502891656 & 0.1332922669223430\end{array}$

$\begin{array}{llll}0.6048225698545684 & 0.4428228759569488 & 0.1300564272345873\end{array}$

$\begin{array}{llll}0.8020637320746086 & 0.4429626537081773 & 0.1331140610119191\end{array}$

$\begin{array}{llll}0.7048231325073422 & 0.2752178281341193 & 0.1326478760181603\end{array}$

$\begin{array}{llll}0.7052831872633996 & 0.6103921625784512 & 0.1327141718758910\end{array}$

$\begin{array}{llll}0.3997140434506543 & 0.1101437714890930 & 0.1383393727854893\end{array}$

$\begin{array}{llll}0.6042504000643407 & 0.1070421481280989 & 0.1311281296841333\end{array}$

$\begin{array}{llll}0.5058454156438422 & 0.2756669504466385 & 0.1332979249864365\end{array}$

$\begin{array}{llll}0.8016758606647750 & 0.1086725076136414 & 0.1328862981376326\end{array}$

$\begin{array}{llll}0.9000545073538492 & 0.2762419476744803 & 0.1334689018693802\end{array}$

$\begin{array}{llll}0.2992371825552181 & 0.9415824850468330 & 0.1316262614814541\end{array}$

$\begin{array}{llll}0.9973362220414906 & 0.7771312535707325 & 0.1330276124392410\end{array}$

$\begin{array}{llll}0.1941400386748783 & 0.7781570026017013 & 0.1311679184364234\end{array}$

$\begin{array}{llll}0.0979036600812371 & 0.9428188660928537 & 0.1332193764532204\end{array}$

$\begin{array}{llll}0.7017885135048565 & 0.9426693026270939 & 0.1331301034650172\end{array}$

$\begin{array}{llll}0.3996687627998275 & 0.7714792230203955 & 0.1382802987620192\end{array}$

$\begin{array}{llll}0.6053541775445386 & 0.7779619096006462 & 0.1312742703640053\end{array}$

$\begin{array}{llll}0.5004349801970506 & 0.9414589977565551 & 0.1315852643498670\end{array}$

$\begin{array}{llll}0.8022829217000840 & 0.7770584778706625 & 0.1330281090136931\end{array}$

$\begin{array}{llll}0.8998888015268051 & 0.9430038746586675 & 0.1329752875919332\end{array}$

$\begin{array}{lllll}0.0000000000000000 & 0.5555400000000006 & 0.0659399999999977\end{array}$

$\begin{array}{lllll}0.1999700000000004 & 0.5555400000000006 & 0.0659399999999977\end{array}$

$\begin{array}{llll}0.0999899999999982 & 0.3888800000000003 & 0.0659399999999977\end{array}$

$\begin{array}{lllll}0.0000000000000000 & 0.2222099999999969 & 0.0659399999999977\end{array}$

$\begin{array}{lllll}0.3999499999999969 & 0.5555400000000006 & 0.0659399999999977\end{array}$

$\begin{array}{lllll}0.5999199999999973 & 0.5555400000000006 & 0.0659399999999977\end{array}$

$\begin{array}{lllll}0.4999299999999991 & 0.3888800000000003 & 0.0659399999999977\end{array}$

$\begin{array}{llll}0.1999700000000004 & 0.2222099999999969 & 0.0659399999999977\end{array}$ 
$\begin{array}{llll}0.3999499999999969 & 0.2222099999999969 & 0.0659399999999977\end{array}$

$\begin{array}{llllll}0.2999599999999987 & 0.0555499999999967 & 0.0659399999999977\end{array}$

$\begin{array}{lllll}0.2999599999999987 & 0.3888800000000003 & 0.0659399999999977\end{array}$

$\begin{array}{lllll}0.0999899999999982 & 0.0555499999999967 & 0.0659399999999977\end{array}$

$\begin{array}{llll}0.7998899999999978 & 0.5555400000000006 & 0.0659399999999977\end{array}$

$\begin{array}{llll}0.8998800000000031 & 0.3888800000000003 & 0.0659399999999977\end{array}$

$\begin{array}{lllll}0.5999199999999973 & 0.2222099999999969 & 0.0659399999999977\end{array}$

$\begin{array}{lllll}0.7998899999999978 & 0.2222099999999969 & 0.0659399999999977\end{array}$

$\begin{array}{lllll}0.6998999999999995 & 0.0555499999999967 & 0.0659399999999977\end{array}$

$\begin{array}{llll}0.6998999999999995 & 0.3888800000000003 & 0.0659399999999977\end{array}$

$\begin{array}{lllll}0.4999299999999991 & 0.0555499999999967 & 0.0659399999999977\end{array}$

$\begin{array}{lllll}0.8998800000000031 & 0.0555499999999967 & 0.0659399999999977\end{array}$

$\begin{array}{llll}0.0000000000000000 & 0.8888800000000003 & 0.0659399999999977\end{array}$

$\begin{array}{llll}0.1999700000000004 & 0.8888800000000003 & 0.0659399999999977\end{array}$

$\begin{array}{llll}0.3999499999999969 & 0.8888800000000003 & 0.0659399999999977\end{array}$

$\begin{array}{lllll}0.2999599999999987 & 0.7222200000000001 & 0.0659399999999977\end{array}$

$\begin{array}{lllll}0.0999899999999982 & 0.7222200000000001 & 0.0659399999999977\end{array}$

$\begin{array}{llll}0.5999199999999973 & 0.8888800000000003 & 0.0659399999999977\end{array}$

$\begin{array}{llll}0.7998899999999978 & 0.8888800000000003 & 0.0659399999999977\end{array}$

$\begin{array}{lllll}0.6998999999999995 & 0.7222200000000001 & 0.0659399999999977\end{array}$

$\begin{array}{lllll}0.4999299999999991 & 0.7222200000000001 & 0.0659399999999977\end{array}$

$\begin{array}{lllll}0.8998800000000031 & 0.7222200000000001 & 0.0659399999999977\end{array}$

$\begin{array}{llll}0.2999599999999987 & 0.4999800000000008 & 0.0000000000000000\end{array}$

$\begin{array}{llll}0.0000000000000000 & 0.3333200000000005 & 0.0000000000000000\end{array}$

0.19997000000000040 .33332000000000050 .0000000000000000

$\begin{array}{lllll}0.0999899999999982 & 0.1666600000000003 & 0.0000000000000000\end{array}$

$\begin{array}{llll}0.0999899999999982 & 0.4999800000000008 & 0.0000000000000000\end{array}$

$\begin{array}{llll}0.0000000000000000 & 0.0000000000000000 & 0.0000000000000000\end{array}$

$\begin{array}{lll}0.6998999999999995 & 0.4999800000000008 & 0.0000000000000000\end{array}$

$\begin{array}{llll}0.3999499999999969 & 0.3333200000000005 & 0.0000000000000000\end{array}$

$\begin{array}{lllll}0.5999199999999973 & 0.3333200000000005 & 0.0000000000000000\end{array}$

$\begin{array}{lllll}0.4999299999999991 & 0.1666600000000003 & 0.0000000000000000\end{array}$

$\begin{array}{llll}0.4999299999999991 & 0.4999800000000008 & 0.0000000000000000\end{array}$

$\begin{array}{lll}0.1999700000000004 & 0.0000000000000000 & 0.0000000000000000\end{array}$

$\begin{array}{lllll}0.3999499999999969 & 0.0000000000000000 & 0.0000000000000000\end{array}$

$\begin{array}{llll}0.2999599999999987 & 0.1666600000000003 & 0.0000000000000000\end{array}$

$\begin{array}{llll}0.7998899999999978 & 0.3333200000000005 & 0.0000000000000000\end{array}$

$\begin{array}{llll}0.8998800000000031 & 0.1666600000000003 & 0.0000000000000000\end{array}$

$\begin{array}{llll}0.8998800000000031 & 0.4999800000000008 & 0.0000000000000000\end{array}$

$\begin{array}{llll}0.5999199999999973 & 0.0000000000000000 & 0.0000000000000000\end{array}$

$\begin{array}{lllll}0.7998899999999978 & 0.0000000000000000 & 0.0000000000000000\end{array}$

$\begin{array}{llll}0.6998999999999995 & 0.1666600000000003 & 0.0000000000000000\end{array}$

$\begin{array}{llll}0.0999899999999982 & 0.8333299999999966 & 0.0000000000000000\end{array}$

$\begin{array}{llll}0.0000000000000000 & 0.6666700000000034 & 0.0000000000000000\end{array}$

$\begin{array}{llll}0.4999299999999991 & 0.8333299999999966 & 0.0000000000000000\end{array}$

0.19997000000000040 .66667000000000340 .0000000000000000

$\begin{array}{llll}0.3999499999999969 & 0.6666700000000034 & 0.0000000000000000\end{array}$ 
$\begin{array}{llll}0.2999599999999987 & 0.8333299999999966 & 0.0000000000000000\end{array}$

$\begin{array}{llll}0.8998800000000031 & 0.8333299999999966 & 0.0000000000000000\end{array}$

$\begin{array}{llll}0.5999199999999973 & 0.6666700000000034 & 0.0000000000000000\end{array}$

$\begin{array}{llll}0.7998899999999978 & 0.6666700000000034 & 0.0000000000000000\end{array}$

$\begin{array}{llll}0.6998999999999995 & 0.8333299999999966 & 0.0000000000000000\end{array}$

$\begin{array}{llll}0.3982728312365011 & 0.4402610542913382 & 0.2023954879162938\end{array}$

$\begin{array}{lllll}0.2566730635480958 & 0.4393903759320023 & 0.2162098163550912\end{array}$

$\begin{array}{llll}0.3981420924579527 & 0.5805706266965959 & 0.2058622444791813\end{array}$

$\begin{array}{llll}0.3985302539297433 & 0.2997236197440355 & 0.2061185722100429\end{array}$

$\begin{array}{llll}0.5394861444996856 & 0.4406629383123508 & 0.2167361702375376\end{array}$

$\begin{array}{llll}0.4497887593174859 & 0.1489532474415468 & 0.1970758721491010\end{array}$

$\begin{array}{llll}0.3486350319908331 & 0.1484182820678212 & 0.1971727500382529\end{array}$

$\begin{array}{llll}0.2259312572850683 & 0.2743593398710958 & 0.2109356219717565\end{array}$

$\begin{array}{llll}0.3188636440171351 & 0.2454575180676383 & 0.2005087848497626\end{array}$

$\begin{array}{llll}0.4786555993776396 & 0.2462313469316550 & 0.2002816271353969\end{array}$

$\begin{array}{llll}0.5713215356857265 & 0.2760508158337470 & 0.2105583782451993\end{array}$

$\begin{array}{llll}0.6923844144205903 & 0.4877891049251631 & 0.2294516304820640\end{array}$

$\begin{array}{llll}0.6930011681989576 & 0.3945044728536065 & 0.2291553627321571\end{array}$

$\begin{array}{llll}0.5978149984569434 & 0.3652331607613734 & 0.2201114715208293\end{array}$

$\begin{array}{lllll}0.5970500031088811 & 0.5166206477377404 & 0.2205308248980657\end{array}$

$\begin{array}{llll}0.5702229511216751 & 0.6057704010469160 & 0.2111488819189627\end{array}$

$\begin{array}{llll}0.3469824917099874 & 0.7314944727201010 & 0.1967466179369896\end{array}$

$\begin{array}{llll}0.4481163327795912 & 0.7321044279575851 & 0.1970359513611286\end{array}$

$\begin{array}{llll}0.4776941180324076 & 0.6349281372539312 & 0.2003673145087766\end{array}$

$\begin{array}{llll}0.3180648652167257 & 0.6339312653986516 & 0.1998183201781250\end{array}$

$\begin{array}{lllll}0.2252582202456901 & 0.6042747099942716 & 0.2104414726422322\end{array}$

$\begin{array}{lllll}0.1988948496209944 & 0.3636347644653898 & 0.2199550645712915\end{array}$

$\begin{array}{llll}0.1035733573942785 & 0.3925891520608363 & 0.2289533154633026\end{array}$

$\begin{array}{llll}0.1033851021775446 & 0.4859140517150598 & 0.2288539603085712\end{array}$

$\begin{array}{llll}0.1985474585132744 & 0.5150794158679832 & 0.2197603416425269\end{array}$

$\begin{array}{llll}0.3039016408729236 & 0.0927538890420663 & 0.2068891705095676\end{array}$

$\begin{array}{llll}0.4952669646488653 & 0.0938397024566215 & 0.2067933203513636\end{array}$

$\begin{array}{llll}0.7530667014107352 & 0.3492001004704116 & 0.2327905685984697\end{array}$

$\begin{array}{llll}0.7520023273925416 & 0.5335069101568846 & 0.2334877101049398\end{array}$

$\begin{array}{llll}0.4928174320286577 & 0.7874346605325770 & 0.2071049728242204\end{array}$

$\begin{array}{lllll}0.3011834897714115 & 0.7864947345085004 & 0.2063117151236872\end{array}$

$\begin{array}{llll}0.0434791824088346 & 0.5313236936611729 & 0.2326320122242153\end{array}$

$\begin{array}{llll}0.0440064816427435 & 0.3468319037402764 & 0.2329095685074148\end{array}$

$\begin{array}{llll}0.1703854732262054 & 0.6564137326985053 & 0.2115323513956433\end{array}$

$\begin{array}{llll}0.1714211077919185 & 0.2218975098391419 & 0.2120708744349304\end{array}$

$\begin{array}{llll}0.6263402857274802 & 0.2240347976435078 & 0.2114521985133746\end{array}$

$\begin{array}{lllll}0.6249231652564681 & 0.6581064817722971 & 0.2124426615155183\end{array}$

FeP-Pt(111) - Hhcp-0, vdW-DF-optPBE:

$\mathrm{Pt} \mathrm{H} \mathrm{C} \mathrm{N} \mathrm{Fe}$

1.00000000000000

$\begin{array}{llll}14.1349999999999998 & 0.0000000000000000 & 0.0000000000000000\end{array}$ 


$\begin{array}{rrrr}0.0000000000000000 & 14.6880000000000006 & 0.0000000000000000 \\ 0.0000000000000000 & 0.0000000000000000 & 35.0000000000000000\end{array}$

$\mathrm{Pt} \quad \mathrm{H} \quad \mathrm{C} \quad \mathrm{N}$ Fe

Direct

$\begin{array}{lllll}90 & 12 & 20 & 4 & 1\end{array}$

$\begin{array}{lll}0.6998999999999995 & 0.8333299999999966 & 0.0000000000000000\end{array}$

$\begin{array}{lllll}0.7998899999999978 & 0.6666700000000034 & 0.0000000000000000\end{array}$

$\begin{array}{lllll}0.5999199999999973 & 0.6666700000000034 & 0.0000000000000000\end{array}$

$\begin{array}{lllll}0.8998800000000031 & 0.8333299999999966 & 0.0000000000000000\end{array}$

$\begin{array}{llll}0.2999599999999987 & 0.8333299999999966 & 0.0000000000000000\end{array}$

$\begin{array}{lll}0.3999499999999969 & 0.6666700000000034 & 0.0000000000000000\end{array}$

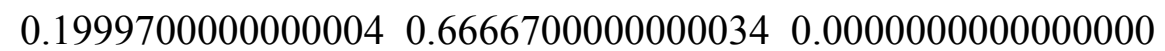

$\begin{array}{llll}0.4999299999999991 & 0.8333299999999966 & 0.0000000000000000\end{array}$

$\begin{array}{llllll}0.0000000000000000 & 0.6666700000000034 & 0.0000000000000000\end{array}$

$\begin{array}{lllll}0.0999899999999982 & 0.8333299999999966 & 0.0000000000000000\end{array}$

$\begin{array}{llll}0.6998999999999995 & 0.1666600000000003 & 0.0000000000000000\end{array}$

$\begin{array}{lllll}0.7998899999999978 & 0.0000000000000000 & 0.0000000000000000\end{array}$

$\begin{array}{lllll}0.5999199999999973 & 0.0000000000000000 & 0.0000000000000000\end{array}$

$\begin{array}{llll}0.8998800000000031 & 0.4999800000000008 & 0.0000000000000000\end{array}$

$\begin{array}{llll}0.8998800000000031 & 0.1666600000000003 & 0.0000000000000000\end{array}$

$\begin{array}{llll}0.7998899999999978 & 0.3333200000000005 & 0.0000000000000000\end{array}$

$\begin{array}{llll}0.2999599999999987 & 0.1666600000000003 & 0.0000000000000000\end{array}$

$\begin{array}{lllll}0.3999499999999969 & 0.0000000000000000 & 0.0000000000000000\end{array}$

$0.19997000000000040 .0000000000000000 \quad 0.0000000000000000$

$\begin{array}{llll}0.4999299999999991 & 0.4999800000000008 & 0.0000000000000000\end{array}$

$\begin{array}{lllll}0.4999299999999991 & 0.1666600000000003 & 0.0000000000000000\end{array}$

$\begin{array}{llll}0.5999199999999973 & 0.3333200000000005 & 0.0000000000000000\end{array}$

$\begin{array}{llll}0.3999499999999969 & 0.3333200000000005 & 0.0000000000000000\end{array}$

$\begin{array}{lllll}0.6998999999999995 & 0.4999800000000008 & 0.0000000000000000\end{array}$

$\begin{array}{lllll}0.0000000000000000 & 0.0000000000000000 & 0.0000000000000000\end{array}$

$\begin{array}{lllll}0.0999899999999982 & 0.4999800000000008 & 0.0000000000000000\end{array}$

$\begin{array}{lllll}0.0999899999999982 & 0.1666600000000003 & 0.0000000000000000\end{array}$

$\begin{array}{lll}0.1999700000000004 & 0.3333200000000005 & 0.0000000000000000\end{array}$

$\begin{array}{llll}0.0000000000000000 & 0.3333200000000005 & 0.0000000000000000\end{array}$

$\begin{array}{lllll}0.2999599999999987 & 0.4999800000000008 & 0.0000000000000000\end{array}$

$\begin{array}{lllll}0.8998800000000031 & 0.7222200000000001 & 0.0659399999999977\end{array}$

$\begin{array}{lllll}0.4999299999999991 & 0.7222200000000001 & 0.0659399999999977\end{array}$

$\begin{array}{lllll}0.6998999999999995 & 0.7222200000000001 & 0.0659399999999977\end{array}$

$\begin{array}{lllll}0.7998899999999978 & 0.8888800000000003 & 0.0659399999999977\end{array}$

$\begin{array}{llll}0.5999199999999973 & 0.8888800000000003 & 0.0659399999999977\end{array}$

$\begin{array}{lllll}0.0999899999999982 & 0.7222200000000001 & 0.0659399999999977\end{array}$

$\begin{array}{lllll}0.2999599999999987 & 0.7222200000000001 & 0.0659399999999977\end{array}$

$\begin{array}{lllll}0.3999499999999969 & 0.8888800000000003 & 0.0659399999999977\end{array}$

$\begin{array}{lllll}0.1999700000000004 & 0.8888800000000003 & 0.0659399999999977\end{array}$

$\begin{array}{llll}0.0000000000000000 & 0.8888800000000003 & 0.0659399999999977\end{array}$

$\begin{array}{llllll}0.8998800000000031 & 0.0555499999999967 & 0.0659399999999977\end{array}$

$\begin{array}{lllll}0.4999299999999991 & 0.0555499999999967 & 0.0659399999999977\end{array}$ 
$\begin{array}{llll}0.6998999999999995 & 0.3888800000000003 & 0.0659399999999977\end{array}$ $\begin{array}{llllll}0.6998999999999995 & 0.0555499999999967 & 0.0659399999999977\end{array}$ $\begin{array}{lllll}0.7998899999999978 & 0.2222099999999969 & 0.0659399999999977\end{array}$ $\begin{array}{lllll}0.5999199999999973 & 0.2222099999999969 & 0.0659399999999977\end{array}$ $\begin{array}{lllll}0.8998800000000031 & 0.3888800000000003 & 0.0659399999999977\end{array}$ $\begin{array}{lllll}0.7998899999999978 & 0.5555400000000006 & 0.0659399999999977\end{array}$ $\begin{array}{lllll}0.0999899999999982 & 0.0555499999999967 & 0.0659399999999977\end{array}$ $\begin{array}{lllll}0.2999599999999987 & 0.3888800000000003 & 0.0659399999999977\end{array}$ $\begin{array}{lllll}0.2999599999999987 & 0.0555499999999967 & 0.0659399999999977\end{array}$ $\begin{array}{llll}0.3999499999999969 & 0.2222099999999969 & 0.0659399999999977\end{array}$ $\begin{array}{lllll}0.1999700000000004 & 0.2222099999999969 & 0.0659399999999977\end{array}$ $\begin{array}{lllll}0.4999299999999991 & 0.3888800000000003 & 0.0659399999999977\end{array}$ $\begin{array}{llll}0.5999199999999973 & 0.5555400000000006 & 0.0659399999999977\end{array}$ $\begin{array}{llll}0.3999499999999969 & 0.5555400000000006 & 0.0659399999999977\end{array}$ $\begin{array}{llll}0.0000000000000000 & 0.2222099999999969 & 0.0659399999999977\end{array}$ $\begin{array}{llll}0.0999899999999982 & 0.3888800000000003 & 0.0659399999999977\end{array}$ $\begin{array}{lllll}0.1999700000000004 & 0.5555400000000006 & 0.0659399999999977\end{array}$ $\begin{array}{llll}0.0000000000000000 & 0.5555400000000006 & 0.0659399999999977\end{array}$ $\begin{array}{llll}0.8999491755192228 & 0.9443360810489937 & 0.1328457977293235\end{array}$ $\begin{array}{llll}0.8016738516168186 & 0.7773633232644533 & 0.1321941378901318\end{array}$ $\begin{array}{llll}0.5004754785720144 & 0.9475062253189065 & 0.1314516855882248\end{array}$ $\begin{array}{llll}0.6024316909557026 & 0.7793223204633513 & 0.1356127655423585\end{array}$ $\begin{array}{llll}0.3999256488600100 & 0.7785263810018339 & 0.1328984711640656\end{array}$ $\begin{array}{llll}0.7015540978577857 & 0.9457361132942984 & 0.1325718147427004\end{array}$ $\begin{array}{llll}0.0983827510953429 & 0.9456092241663615 & 0.1325755381023377\end{array}$ $\begin{array}{llll}0.1974034986734147 & 0.7792612561639033 & 0.1356113300985055\end{array}$ $\begin{array}{llll}0.9979643984089719 & 0.7775449891418830 & 0.1321607026720793\end{array}$ $\begin{array}{llll}0.2990293188826993 & 0.9475354663861232 & 0.1315071605700730\end{array}$ $\begin{array}{llll}0.8995918904716005 & 0.2755063597958645 & 0.1328549129331482\end{array}$ $\begin{array}{llll}0.8002334878388638 & 0.1098108031724458 & 0.1329001308276143\end{array}$ $\begin{array}{llll}0.4998332684462741 & 0.2764104214977618 & 0.1327183563649865\end{array}$ $\begin{array}{llll}0.6005571108799838 & 0.1106000907158486 & 0.1330447530936868\end{array}$ $\begin{array}{llll}0.3998179680750222 & 0.1114898008225488 & 0.1332207595199840\end{array}$ $\begin{array}{llll}0.7021723657485200 & 0.6086544440409621 & 0.1318253539554581\end{array}$ $\begin{array}{llll}0.7004954606346643 & 0.2755560844927589 & 0.1327265874103770\end{array}$ $\begin{array}{llll}0.8011537478972739 & 0.4419646094223597 & 0.1327745653704055\end{array}$ $\begin{array}{llll}0.6017229644473264 & 0.4411478642893148 & 0.1317723722335659\end{array}$ $\begin{array}{llll}0.8999043451431629 & 0.6092498210550878 & 0.1328882255226103\end{array}$ $\begin{array}{llll}0.0990395769935943 & 0.2755517050924641 & 0.1327227496583559\end{array}$ $\begin{array}{llll}0.1990303145640309 & 0.1107416374145543 & 0.1330267987347432\end{array}$ $\begin{array}{llll}0.9995739416788556 & 0.1098848625593980 & 0.1328676243831106\end{array}$ $\begin{array}{llll}0.2990007183076646 & 0.6063029023329418 & 0.1308143910722548\end{array}$ $\begin{array}{llll}0.2998711461030937 & 0.2763939365452093 & 0.1326576743013139\end{array}$ $\begin{array}{llll}0.3995458667485408 & 0.4429837824112151 & 0.1339729066492627\end{array}$ $\begin{array}{llll}0.1974087059089931 & 0.4410325442309423 & 0.1319749779586271\end{array}$ $\begin{array}{llll}0.4993222227817472 & 0.6064918843944410 & 0.1310095311479618\end{array}$ $\begin{array}{llll}0.9984507667955885 & 0.4418613392551329 & 0.1327985611194459\end{array}$ 
$\begin{array}{llll}0.0971084324407748 & 0.6089605721773746 & 0.1318310077871852\end{array}$ $\begin{array}{llll}0.3995124938691802 & 0.8526597216062899 & 0.2008547096923041\end{array}$

$\begin{array}{llll}0.7235607077998195 & 0.5482170702536066 & 0.2202421519714207\end{array}$ $\begin{array}{llll}0.4041024863672733 & 0.2385786983598439 & 0.2279440496783528\end{array}$ $\begin{array}{llll}0.0801577675263659 & 0.5432677889355465 & 0.2196623474203272\end{array}$ $\begin{array}{llll}0.2162127001065659 & 0.2349268247260532 & 0.2278947149925831\end{array}$ $\begin{array}{llll}0.0795316155649547 & 0.3641987188209667 & 0.2239544775602312\end{array}$ $\begin{array}{llll}0.0742427896153512 & 0.7240450856924363 & 0.2122996748972557\end{array}$ $\begin{array}{llll}0.2092780208589063 & 0.8545403441366164 & 0.2054650175409362\end{array}$ $\begin{array}{llll}0.5887563275679184 & 0.8574246993265757 & 0.2049908721221457\end{array}$ $\begin{array}{llll}0.7261892300967399 & 0.7295563718804147 & 0.2124008665871186\end{array}$ $\begin{array}{llll}0.7269526759470814 & 0.3687286383265834 & 0.2243227711046090\end{array}$ $\begin{array}{llll}0.5924578499718394 & 0.2374835971531902 & 0.2280022085777736\end{array}$ $\begin{array}{llll}0.2033635316742951 & 0.4607611651797552 & 0.2213832745683380\end{array}$ $\begin{array}{llll}0.1557437479574152 & 0.3734759063070762 & 0.2242854321887976\end{array}$ $\begin{array}{llll}0.2247815917166235 & 0.3083045016493154 & 0.2261982824865293\end{array}$ $\begin{array}{llll}0.3149550453677444 & 0.3551582695985430 & 0.2247418828685245\end{array}$ $\begin{array}{llll}0.1573053016289320 & 0.5447233938110249 & 0.2189605408967990\end{array}$ $\begin{array}{llll}0.1992271478668350 & 0.6305506493034673 & 0.2136365135788513\end{array}$ $\begin{array}{llll}0.3099392014010069 & 0.7352475458637624 & 0.2028973826717466\end{array}$ $\begin{array}{llll}0.2181032166177721 & 0.7821614409721301 & 0.1995383642451639\end{array}$ $\begin{array}{llll}0.1496991731023238 & 0.7137894386842697 & 0.2084253719364457\end{array}$ $\begin{array}{llll}0.3999026029930918 & 0.7789386453185465 & 0.1962164141780445\end{array}$ $\begin{array}{llll}0.4903108678788614 & 0.7362193339513785 & 0.2030258793914835\end{array}$ $\begin{array}{llll}0.6031458455590331 & 0.6336971781638996 & 0.2140788221453954\end{array}$ $\begin{array}{llll}0.6508413420565253 & 0.7180393502114069 & 0.2087856400993119\end{array}$ $\begin{array}{llll}0.5812520026829532 & 0.7846229834827836 & 0.1996691614605267\end{array}$ $\begin{array}{llll}0.6463774037012423 & 0.5484459513260188 & 0.2192833424096354\end{array}$ $\begin{array}{llll}0.6016121561035630 & 0.4637121886850283 & 0.2215844323902702\end{array}$ $\begin{array}{llll}0.4917259976751336 & 0.3562567127066903 & 0.2248462222474470\end{array}$ $\begin{array}{llll}0.4036340011788000 & 0.3126769050199283 & 0.2259821441900050\end{array}$ $\begin{array}{llll}0.5826630446997977 & 0.3107378949153088 & 0.2264440416631213\end{array}$ $\begin{array}{llll}0.6505402635772063 & 0.3768976223543833 & 0.2244429594639353\end{array}$ $\begin{array}{llll}0.3006929307719904 & 0.4479643778174654 & 0.2217132948763921\end{array}$ $\begin{array}{llll}0.2973491794877631 & 0.6450422815342308 & 0.2096596849764757\end{array}$ $\begin{array}{llll}0.5045473482185023 & 0.4493035495082787 & 0.2218149791018266\end{array}$ $\begin{array}{lllll}0.5046655822850898 & 0.6464273872089471 & 0.2100779768664280\end{array}$ $\begin{array}{llll}0.4020766339852477 & 0.5386625804870119 & 0.2018312594091024\end{array}$

FeP-Pt(111) - Hhcp-45, vdW-DF-optPBE:

Pt H C N Fe

1.00000000000000

$\begin{array}{llll}14.1349999999999998 & 0.0000000000000000 & 0.0000000000000000\end{array}$ $\begin{array}{lll}0.0000000000000000 & 14.6880000000000006 & 0.0000000000000000\end{array}$ $\begin{array}{llll}0.0000000000000000 & 0.0000000000000000 & 35.0000000000000000\end{array}$ $\mathrm{Pt} \mathrm{H} \quad \mathrm{C} \quad \mathrm{N}$ Fe $\begin{array}{lllll}90 & 12 & 20 & 4 & 1\end{array}$ 
Direct

0.69989999999999950 .83332999999999660 .0000000000000000

$\begin{array}{llll}0.7998899999999978 & 0.6666700000000034 & 0.0000000000000000\end{array}$

$\begin{array}{lll}0.5999199999999973 & 0.6666700000000034 & 0.0000000000000000\end{array}$

$\begin{array}{llll}0.8998800000000031 & 0.8333299999999966 & 0.0000000000000000\end{array}$

$\begin{array}{llll}0.2999599999999987 & 0.8333299999999966 & 0.0000000000000000\end{array}$

$\begin{array}{llll}0.3999499999999969 & 0.6666700000000034 & 0.0000000000000000\end{array}$

0.19997000000000040 .66667000000000340 .0000000000000000

$\begin{array}{llll}0.4999299999999991 & 0.8333299999999966 & 0.0000000000000000\end{array}$

$\begin{array}{llll}0.0000000000000000 & 0.6666700000000034 & 0.0000000000000000\end{array}$

$\begin{array}{lllll}0.0999899999999982 & 0.8333299999999966 & 0.0000000000000000\end{array}$

$\begin{array}{llll}0.6998999999999995 & 0.1666600000000003 & 0.0000000000000000\end{array}$

$\begin{array}{llll}0.7998899999999978 & 0.0000000000000000 & 0.0000000000000000\end{array}$

$\begin{array}{llll}0.5999199999999973 & 0.0000000000000000 & 0.0000000000000000\end{array}$

$\begin{array}{llll}0.8998800000000031 & 0.4999800000000008 & 0.0000000000000000\end{array}$

$\begin{array}{llll}0.8998800000000031 & 0.1666600000000003 & 0.0000000000000000\end{array}$

$\begin{array}{lllll}0.7998899999999978 & 0.3333200000000005 & 0.0000000000000000\end{array}$

$\begin{array}{llll}0.2999599999999987 & 0.1666600000000003 & 0.0000000000000000\end{array}$

$\begin{array}{llll}0.3999499999999969 & 0.0000000000000000 & 0.0000000000000000\end{array}$

$\begin{array}{lll}0.1999700000000004 & 0.0000000000000000 & 0.0000000000000000\end{array}$

$\begin{array}{lllll}0.4999299999999991 & 0.4999800000000008 & 0.0000000000000000\end{array}$

$\begin{array}{llll}0.4999299999999991 & 0.1666600000000003 & 0.0000000000000000\end{array}$

$\begin{array}{llll}0.5999199999999973 & 0.3333200000000005 & 0.0000000000000000\end{array}$

$\begin{array}{llll}0.3999499999999969 & 0.3333200000000005 & 0.0000000000000000\end{array}$

$\begin{array}{lll}0.6998999999999995 & 0.4999800000000008 & 0.0000000000000000\end{array}$

$\begin{array}{lllll}0.0000000000000000 & 0.0000000000000000 & 0.0000000000000000\end{array}$

$\begin{array}{llll}0.0999899999999982 & 0.4999800000000008 & 0.0000000000000000\end{array}$

$\begin{array}{llll}0.0999899999999982 & 0.1666600000000003 & 0.0000000000000000\end{array}$

$\begin{array}{llll}0.1999700000000004 & 0.3333200000000005 & 0.0000000000000000\end{array}$

$\begin{array}{llll}0.0000000000000000 & 0.3333200000000005 & 0.0000000000000000\end{array}$

$\begin{array}{llll}0.2999599999999987 & 0.4999800000000008 & 0.0000000000000000\end{array}$

$\begin{array}{lllll}0.8998800000000031 & 0.7222200000000001 & 0.0659399999999977\end{array}$

$\begin{array}{lllll}0.4999299999999991 & 0.7222200000000001 & 0.0659399999999977\end{array}$

$\begin{array}{lllll}0.6998999999999995 & 0.7222200000000001 & 0.0659399999999977\end{array}$

$\begin{array}{lllll}0.7998899999999978 & 0.8888800000000003 & 0.0659399999999977\end{array}$

$\begin{array}{lllll}0.5999199999999973 & 0.8888800000000003 & 0.0659399999999977\end{array}$

$\begin{array}{lllll}0.0999899999999982 & 0.7222200000000001 & 0.0659399999999977\end{array}$

$\begin{array}{lllll}0.2999599999999987 & 0.7222200000000001 & 0.0659399999999977\end{array}$

$\begin{array}{llll}0.3999499999999969 & 0.8888800000000003 & 0.0659399999999977\end{array}$

$\begin{array}{llll}0.1999700000000004 & 0.8888800000000003 & 0.0659399999999977\end{array}$

$\begin{array}{lllll}0.0000000000000000 & 0.8888800000000003 & 0.0659399999999977\end{array}$

$\begin{array}{lllll}0.8998800000000031 & 0.0555499999999967 & 0.0659399999999977\end{array}$

$\begin{array}{lllll}0.4999299999999991 & 0.0555499999999967 & 0.0659399999999977\end{array}$

$\begin{array}{llll}0.6998999999999995 & 0.3888800000000003 & 0.0659399999999977\end{array}$

$\begin{array}{llllll}0.6998999999999995 & 0.0555499999999967 & 0.0659399999999977\end{array}$

$\begin{array}{lllll}0.7998899999999978 & 0.2222099999999969 & 0.0659399999999977\end{array}$

$\begin{array}{lllll}0.5999199999999973 & 0.2222099999999969 & 0.0659399999999977\end{array}$ 
$\begin{array}{llll}0.8998800000000031 & 0.3888800000000003 & 0.0659399999999977\end{array}$

$\begin{array}{llll}0.7998899999999978 & 0.5555400000000006 & 0.0659399999999977\end{array}$

$\begin{array}{lllll}0.0999899999999982 & 0.0555499999999967 & 0.0659399999999977\end{array}$

$\begin{array}{lllll}0.2999599999999987 & 0.3888800000000003 & 0.0659399999999977\end{array}$

$\begin{array}{lllll}0.2999599999999987 & 0.0555499999999967 & 0.0659399999999977\end{array}$

$\begin{array}{lllll}0.3999499999999969 & 0.2222099999999969 & 0.0659399999999977\end{array}$

$\begin{array}{lllll}0.1999700000000004 & 0.2222099999999969 & 0.0659399999999977\end{array}$

$\begin{array}{lllll}0.4999299999999991 & 0.3888800000000003 & 0.0659399999999977\end{array}$

$\begin{array}{llll}0.5999199999999973 & 0.5555400000000006 & 0.0659399999999977\end{array}$

$\begin{array}{llll}0.3999499999999969 & 0.5555400000000006 & 0.0659399999999977\end{array}$

$\begin{array}{llll}0.0000000000000000 & 0.2222099999999969 & 0.0659399999999977\end{array}$

$\begin{array}{lllll}0.0999899999999982 & 0.3888800000000003 & 0.0659399999999977\end{array}$

$\begin{array}{lllll}0.1999700000000004 & 0.5555400000000006 & 0.0659399999999977\end{array}$

$\begin{array}{llll}0.0000000000000000 & 0.5555400000000006 & 0.0659399999999977\end{array}$

$\begin{array}{llll}0.8998398928808147 & 0.9399385024531459 & 0.1330411462292224\end{array}$

$\begin{array}{llll}0.8003290866803018 & 0.7763772630212017 & 0.1320710902587813\end{array}$

$\begin{array}{llll}0.5021916546868078 & 0.9380593397028404 & 0.1327985175579511\end{array}$

$\begin{array}{llll}0.5984312116012017 & 0.7722907847215232 & 0.1382422984960200\end{array}$

$\begin{array}{llll}0.3999617287088650 & 0.7751010274270608 & 0.1293505555436926\end{array}$

$\begin{array}{llll}0.7003055483712182 & 0.9387906172786732 & 0.1333440852663739\end{array}$

$\begin{array}{llll}0.0994434743617243 & 0.9387558229112066 & 0.1332642080066516\end{array}$

$\begin{array}{llll}0.2011106934029134 & 0.7719740767835404 & 0.1381784471390793\end{array}$

$\begin{array}{llll}0.9994388129153691 & 0.7762966498619941 & 0.1320382465585207\end{array}$

$\begin{array}{llll}0.2977129444222777 & 0.9380252582809737 & 0.1327827967887230\end{array}$

$\begin{array}{llll}0.8998721392027276 & 0.2700186113961128 & 0.1330504450664687\end{array}$

$\begin{array}{lllll}0.8008587394090847 & 0.1041943714386222 & 0.1329909134648595\end{array}$

$\begin{array}{llll}0.4989380764586014 & 0.2640308726455355 & 0.1344403007196172\end{array}$

$\begin{array}{llll}0.6023365004570168 & 0.1017008513342008 & 0.1327899932285282\end{array}$

$\begin{array}{llll}0.3999549176072400 & 0.0987515134737654 & 0.1323478782205932\end{array}$

$\begin{array}{llll}0.7059602198163257 & 0.6098190149622156 & 0.1357452214977854\end{array}$

$\begin{array}{llll}0.7019162499362320 & 0.2673492126578580 & 0.1321064002190538\end{array}$

$\begin{array}{lllll}0.8004218927036015 & 0.4367372637322677 & 0.1319975817795327\end{array}$

$\begin{array}{llll}0.6004617478710291 & 0.4301052557043602 & 0.1345525267353275\end{array}$

$\begin{array}{llll}0.8998799020975344 & 0.6058488409782031 & 0.1314251572370994\end{array}$

$\begin{array}{llll}0.0979845964415914 & 0.2673151746045450 & 0.1320987998749546\end{array}$

$\begin{array}{llll}0.1975008956080232 & 0.1016693535827335 & 0.1327893114683627\end{array}$

$\begin{array}{llll}0.9989729103689582 & 0.1042923770534167 & 0.1329482076498861\end{array}$

$\begin{array}{llll}0.2979928440135788 & 0.6073870304906270 & 0.1296101751008832\end{array}$

$\begin{array}{lllll}0.3011252823982957 & 0.2639964780062527 & 0.1344494778752894\end{array}$

$\begin{array}{llll}0.3999339447624915 & 0.4435568395188579 & 0.1300883129856428\end{array}$

$\begin{array}{llll}0.1994931136816041 & 0.4299033944285758 & 0.1345843769926370\end{array}$

$\begin{array}{llll}0.5019637022830052 & 0.6074173977053028 & 0.1296328025622701\end{array}$

$\begin{array}{llll}0.9995105159207313 & 0.4365869479584371 & 0.1320313518484587\end{array}$

$\begin{array}{llll}0.0937875610701307 & 0.6093290888031678 & 0.1356145150143110\end{array}$

$\begin{array}{llll}0.1700097445626559 & 0.7932794011247557 & 0.2115440567641289\end{array}$

$\begin{array}{llll}0.6280756547841810 & 0.7944062681252909 & 0.2121050483038655\end{array}$

$\begin{array}{llll}0.6279626480755249 & 0.3562987212555409 & 0.2049271134046141\end{array}$ 
$\begin{array}{llll}0.1721922328269474 & 0.3555973711392215 & 0.2048869999779005\end{array}$

$\begin{array}{llll}0.4922670453722892 & 0.2259131853846806 & 0.2086199796687468\end{array}$

$\begin{array}{llll}0.3083052297032227 & 0.2256333548402095 & 0.2086227572524360\end{array}$

$\begin{array}{llll}0.0387942251736042 & 0.4795691350592013 & 0.2100097344227676\end{array}$

$\begin{array}{llll}0.0371768385032827 & 0.6660543254853243 & 0.2072714757497136\end{array}$

$\begin{array}{llll}0.3022886703706443 & 0.9209923773889752 & 0.2263310629029608\end{array}$

$\begin{array}{llll}0.4949631139007952 & 0.9215640311516747 & 0.2267531209020460\end{array}$

$\begin{array}{llll}0.7615403019063736 & 0.6674950484091778 & 0.2074441042545400\end{array}$

$\begin{array}{llll}0.7609620956749743 & 0.4809903288688316 & 0.2097512560278454\end{array}$

$\begin{array}{llll}0.3204894240815637 & 0.3747346438077436 & 0.1985935695304450\end{array}$

$\begin{array}{llll}0.3471944195785343 & 0.2791549455616428 & 0.1936911627054016\end{array}$

$\begin{array}{llll}0.4531050551295810 & 0.2792437862123661 & 0.1936847176900487\end{array}$

$\begin{array}{llll}0.4795855478956902 & 0.3748989597045935 & 0.1985766710643555\end{array}$

$\begin{array}{llll}0.2252407123734972 & 0.4081861356333940 & 0.1994323800257962\end{array}$

$\begin{array}{llll}0.1951672592203964 & 0.4996995878590288 & 0.2080874247378537\end{array}$

$\begin{array}{lllll}0.1951320155541701 & 0.6508657785762020 & 0.2040856590317422\end{array}$

$\begin{array}{llll}0.0965089265115648 & 0.6212169358350501 & 0.2009464657615893\end{array}$

$\begin{array}{llll}0.0989258945435406 & 0.5253257570360703 & 0.2076867850375181\end{array}$

$\begin{array}{llll}0.2250269009323474 & 0.7449391161671550 & 0.2030340723010982\end{array}$

$\begin{array}{llll}0.3202765038394338 & 0.7735879506195025 & 0.2120748875024803\end{array}$

$\begin{array}{llll}0.4780381002259090 & 0.7740506579740513 & 0.2124090108168647\end{array}$

$\begin{array}{llll}0.4480983048271632 & 0.8643070847444287 & 0.2216125272347920\end{array}$

$\begin{array}{llll}0.3496786403358763 & 0.8639544934110510 & 0.2214145180946144\end{array}$

$\begin{array}{llll}0.5733349220032113 & 0.7456689405761674 & 0.2037645128762107\end{array}$

$\begin{array}{llll}0.6036764497249026 & 0.6517373368858060 & 0.2044986083207187\end{array}$

$\begin{array}{llll}0.6044289895235460 & 0.5003119170697858 & 0.2080243465599494\end{array}$

$\begin{array}{llll}0.5747061963784506 & 0.4086515152034323 & 0.1994116275083570\end{array}$

$\begin{array}{llll}0.7005460331993247 & 0.5264315461643903 & 0.2075528964215371\end{array}$

$\begin{array}{llll}0.7023137934734979 & 0.6225388692407822 & 0.2011311722738322\end{array}$

$\begin{array}{llll}0.3999616492891505 & 0.4307787506099783 & 0.1984950931914023\end{array}$

$\begin{array}{llll}0.2533524965524379 & 0.5767400682372509 & 0.2076922692376235\end{array}$

$\begin{array}{llll}0.5458662588199107 & 0.5771502568683650 & 0.2078504367342209\end{array}$

$\begin{array}{llll}0.3992950070096910 & 0.7186186581643644 & 0.2077882638342069\end{array}$

$\begin{array}{llll}0.3996542943001236 & 0.5798054510558942 & 0.1965852412682268\end{array}$

\section{FeP-Pt(111) - Hfcc-0, vdW-DF-optPBE:}

$\mathrm{Pt} \mathrm{Fe} \mathrm{N} \mathrm{C} \mathrm{H}$

1.00000000000000

$\begin{array}{lll}14.1349999999999998 & 0.0000000000000000 & 0.0000000000000000\end{array}$

$\begin{array}{lll}0.0000000000000000 & 14.6880000000000006 & 0.0000000000000000\end{array}$

$\begin{array}{llll}0.0000000000000000 & 0.0000000000000000 & 35.0000000000000000\end{array}$

$\mathrm{Pt} F \mathrm{~N}$ C $\mathrm{H}$

$\begin{array}{lllll}90 & 1 & 4 & 20 & 12\end{array}$

Direct

$\begin{array}{llll}0.0980339621386605 & 0.6093053149318661 & 0.1329013134894078\end{array}$

$\begin{array}{llll}0.9999983651023846 & 0.4425805592965642 & 0.1329774631305572\end{array}$

$\begin{array}{llll}0.4999923306207301 & 0.6102430259982049 & 0.1341122974608736\end{array}$ 
$\begin{array}{llll}0.1987962242224881 & 0.4427303174255959 & 0.1318406083001034\end{array}$ $\begin{array}{llll}0.3998904577770335 & 0.4471250325897723 & 0.1306419834116344\end{array}$ $\begin{array}{llll}0.2991373443804296 & 0.2712072129216577 & 0.1357610569591229\end{array}$ $\begin{array}{llll}0.2959947822623452 & 0.6108956759828637 & 0.1321216509292983\end{array}$ $\begin{array}{llll}0.0000299967434073 & 0.1073559443900548 & 0.1326833992381150\end{array}$ $\begin{array}{llll}0.1982811874053425 & 0.1060148327251757 & 0.1323194117213765\end{array}$ $\begin{array}{llll}0.0988679182675967 & 0.2744798974652550 & 0.1320246467420877\end{array}$ $\begin{array}{llll}0.9020389267728998 & 0.6092474259556043 & 0.1329276031759580\end{array}$ $\begin{array}{llll}0.6003513446781921 & 0.4471043501651815 & 0.1306985154790759\end{array}$ $\begin{array}{llll}0.8012271988512759 & 0.4426717529037442 & 0.1318658188230830\end{array}$ $\begin{array}{llll}0.7008763029818182 & 0.2713504629325044 & 0.1358939461658883\end{array}$ $\begin{array}{llll}0.7040524700309142 & 0.6108184436534655 & 0.1321778108286432\end{array}$ $\begin{array}{llll}0.3997218795533058 & 0.1032227695516663 & 0.1316242691922040\end{array}$ $\begin{array}{llll}0.6003624640943831 & 0.1033185981694538 & 0.1316479655076002\end{array}$ $\begin{array}{llll}0.5000271830704778 & 0.2717903073964563 & 0.1329098514951349\end{array}$ $\begin{array}{llll}0.8017542686386999 & 0.1061178242089189 & 0.1323564254380797\end{array}$ $\begin{array}{llll}0.9010724269712248 & 0.2744886652926163 & 0.1320424369634919\end{array}$ $\begin{array}{llll}0.2994839406868613 & 0.9404417798281974 & 0.1329446690955382\end{array}$ $\begin{array}{llll}0.0000230519524968 & 0.7757099159678594 & 0.1332617663565789\end{array}$ $\begin{array}{llll}0.1987971749342188 & 0.7757057525942423 & 0.1327671719451101\end{array}$ $\begin{array}{llll}0.0997831503635637 & 0.9408979824848294 & 0.1328285398785843\end{array}$ $\begin{array}{llll}0.7006950743508423 & 0.9403913867768821 & 0.1329459935552846\end{array}$ $\begin{array}{llll}0.3999224555252718 & 0.7748092510498132 & 0.1325094415458030\end{array}$ $\begin{array}{llll}0.6002171705139993 & 0.7748013407986224 & 0.1325272899185961\end{array}$ $\begin{array}{lllll}0.5000647016240779 & 0.9389411011206310 & 0.1333327975203225\end{array}$ $\begin{array}{llll}0.8012353675312521 & 0.7755507398586177 & 0.1327765610817398\end{array}$ $\begin{array}{lllll}0.9002885376100692 & 0.9409332084751101 & 0.1328109369418236\end{array}$ $\begin{array}{lllll}0.0000000000000000 & 0.5555400000000006 & 0.0659399999999977\end{array}$ $\begin{array}{llll}0.1999700000000004 & 0.5555400000000006 & 0.0659399999999977\end{array}$ $\begin{array}{lllll}0.0999899999999982 & 0.3888800000000003 & 0.0659399999999977\end{array}$ $\begin{array}{lllll}0.0000000000000000 & 0.2222099999999969 & 0.0659399999999977\end{array}$ $\begin{array}{lllll}0.3999499999999969 & 0.5555400000000006 & 0.0659399999999977\end{array}$ $\begin{array}{lllll}0.5999199999999973 & 0.5555400000000006 & 0.0659399999999977\end{array}$ $\begin{array}{llll}0.4999299999999991 & 0.3888800000000003 & 0.0659399999999977\end{array}$ $\begin{array}{lllll}0.1999700000000004 & 0.2222099999999969 & 0.0659399999999977\end{array}$ $\begin{array}{lllll}0.3999499999999969 & 0.2222099999999969 & 0.0659399999999977\end{array}$ $\begin{array}{lllll}0.2999599999999987 & 0.0555499999999967 & 0.0659399999999977\end{array}$ $\begin{array}{llll}0.2999599999999987 & 0.3888800000000003 & 0.0659399999999977\end{array}$ $\begin{array}{lllll}0.0999899999999982 & 0.0555499999999967 & 0.0659399999999977\end{array}$ $\begin{array}{lllll}0.7998899999999978 & 0.5555400000000006 & 0.0659399999999977\end{array}$ $\begin{array}{lllll}0.8998800000000031 & 0.3888800000000003 & 0.0659399999999977\end{array}$ $\begin{array}{lllll}0.5999199999999973 & 0.2222099999999969 & 0.0659399999999977\end{array}$ $\begin{array}{llll}0.7998899999999978 & 0.2222099999999969 & 0.0659399999999977\end{array}$ $\begin{array}{lllll}0.6998999999999995 & 0.0555499999999967 & 0.0659399999999977\end{array}$ $\begin{array}{lllll}0.6998999999999995 & 0.3888800000000003 & 0.0659399999999977\end{array}$ $\begin{array}{lllll}0.4999299999999991 & 0.0555499999999967 & 0.0659399999999977\end{array}$ $\begin{array}{llll}0.8998800000000031 & 0.0555499999999967 & 0.0659399999999977\end{array}$ 
$\begin{array}{llll}0.0000000000000000 & 0.8888800000000003 & 0.0659399999999977\end{array}$

$\begin{array}{lllll}0.1999700000000004 & 0.8888800000000003 & 0.0659399999999977\end{array}$

$\begin{array}{llll}0.3999499999999969 & 0.8888800000000003 & 0.0659399999999977\end{array}$

$\begin{array}{lllll}0.2999599999999987 & 0.7222200000000001 & 0.0659399999999977\end{array}$

$\begin{array}{lllll}0.0999899999999982 & 0.7222200000000001 & 0.0659399999999977\end{array}$

$\begin{array}{llll}0.5999199999999973 & 0.8888800000000003 & 0.0659399999999977\end{array}$

$\begin{array}{lllll}0.7998899999999978 & 0.8888800000000003 & 0.0659399999999977\end{array}$

$\begin{array}{lllll}0.6998999999999995 & 0.7222200000000001 & 0.0659399999999977\end{array}$

$\begin{array}{lllll}0.4999299999999991 & 0.7222200000000001 & 0.0659399999999977\end{array}$

$\begin{array}{llll}0.8998800000000031 & 0.7222200000000001 & 0.0659399999999977\end{array}$

$\begin{array}{llll}0.2999599999999987 & 0.4999800000000008 & 0.0000000000000000\end{array}$

$\begin{array}{llll}0.0000000000000000 & 0.3333200000000005 & 0.0000000000000000\end{array}$

$\begin{array}{llll}0.1999700000000004 & 0.3333200000000005 & 0.0000000000000000\end{array}$

$\begin{array}{llll}0.0999899999999982 & 0.1666600000000003 & 0.0000000000000000\end{array}$

$\begin{array}{llll}0.0999899999999982 & 0.4999800000000008 & 0.0000000000000000\end{array}$

$\begin{array}{llll}0.0000000000000000 & 0.0000000000000000 & 0.0000000000000000\end{array}$

$\begin{array}{llll}0.6998999999999995 & 0.4999800000000008 & 0.0000000000000000\end{array}$

$\begin{array}{llll}0.3999499999999969 & 0.3333200000000005 & 0.0000000000000000\end{array}$

$\begin{array}{lll}0.5999199999999973 & 0.3333200000000005 & 0.0000000000000000\end{array}$

$\begin{array}{llll}0.4999299999999991 & 0.1666600000000003 & 0.0000000000000000\end{array}$

$\begin{array}{llll}0.4999299999999991 & 0.4999800000000008 & 0.0000000000000000\end{array}$

$\begin{array}{lllll}0.1999700000000004 & 0.0000000000000000 & 0.0000000000000000\end{array}$

$\begin{array}{llll}0.3999499999999969 & 0.0000000000000000 & 0.0000000000000000\end{array}$

$\begin{array}{llll}0.2999599999999987 & 0.1666600000000003 & 0.0000000000000000\end{array}$

$\begin{array}{llll}0.7998899999999978 & 0.3333200000000005 & 0.0000000000000000\end{array}$

$\begin{array}{lllll}0.8998800000000031 & 0.1666600000000003 & 0.0000000000000000\end{array}$

$\begin{array}{llll}0.8998800000000031 & 0.4999800000000008 & 0.0000000000000000\end{array}$

$\begin{array}{llll}0.5999199999999973 & 0.0000000000000000 & 0.0000000000000000\end{array}$

$\begin{array}{lllll}0.7998899999999978 & 0.0000000000000000 & 0.0000000000000000\end{array}$

$\begin{array}{llll}0.6998999999999995 & 0.1666600000000003 & 0.0000000000000000\end{array}$

$\begin{array}{lllll}0.0999899999999982 & 0.8333299999999966 & 0.0000000000000000\end{array}$

$\begin{array}{llll}0.0000000000000000 & 0.6666700000000034 & 0.0000000000000000\end{array}$

$\begin{array}{llll}0.4999299999999991 & 0.8333299999999966 & 0.0000000000000000\end{array}$

0.19997000000000040 .66667000000000340 .0000000000000000

$\begin{array}{lllll}0.3999499999999969 & 0.6666700000000034 & 0.0000000000000000\end{array}$

$\begin{array}{lllll}0.2999599999999987 & 0.8333299999999966 & 0.0000000000000000\end{array}$

$\begin{array}{llll}0.8998800000000031 & 0.8333299999999966 & 0.0000000000000000\end{array}$

$\begin{array}{lll}0.5999199999999973 & 0.6666700000000034 & 0.0000000000000000\end{array}$

$\begin{array}{llll}0.7998899999999978 & 0.6666700000000034 & 0.0000000000000000\end{array}$

$\begin{array}{lllll}0.6998999999999995 & 0.8333299999999966 & 0.0000000000000000\end{array}$

$\begin{array}{llll}0.4990439725775322 & 0.5186311427139172 & 0.1994844541625179\end{array}$

$\begin{array}{llll}0.3971509021387974 & 0.6072317060565507 & 0.2206346487956464\end{array}$

$\begin{array}{llll}0.6001166478472927 & 0.6080282734144049 & 0.2209251424209328\end{array}$

$\begin{array}{llll}0.3957405755821276 & 0.4104248021206268 & 0.2082164384908740\end{array}$

$\begin{array}{lllll}0.6026035752383474 & 0.4110536637000471 & 0.2084145775184499\end{array}$

$\begin{array}{llll}0.6769452292641933 & 0.7470625442702814 & 0.2265233950491500\end{array}$

$\begin{array}{llll}0.7454308498633154 & 0.6815053907645615 & 0.2246089400523125\end{array}$ 
$\begin{array}{llll}0.7427584113579490 & 0.5104716492074596 & 0.2189589400358490\end{array}$

$\begin{array}{llll}0.6973289348757652 & 0.5946397669864456 & 0.2210612405785153\end{array}$

$\begin{array}{lllll}0.5865275972000369 & 0.7007719357362124 & 0.2244331679521701\end{array}$

$\begin{array}{llll}0.4981910517836380 & 0.7436105303742732 & 0.2261316793401842\end{array}$

$\begin{array}{llll}0.2513940631812717 & 0.6798778904050752 & 0.2246209627530395\end{array}$

$\begin{array}{llll}0.3196185766884166 & 0.7456439425084354 & 0.2268530783402408\end{array}$

$\begin{array}{lllll}0.4103066566757045 & 0.7000228709619546 & 0.2244555307775355\end{array}$

$\begin{array}{lllll}0.2999542598374274 & 0.5932211523364536 & 0.2206073469388533\end{array}$

$\begin{array}{llll}0.2547655886402320 & 0.5088781631630792 & 0.2184247637882760\end{array}$

$\begin{array}{llll}0.3185435727908550 & 0.2714005393517118 & 0.1996496980076737\end{array}$

$\begin{array}{llll}0.2494102702266458 & 0.3388561289925552 & 0.2087912965707599\end{array}$

$\begin{array}{llll}0.2974246742828643 & 0.4233143004428654 & 0.2130271434100910\end{array}$

$\begin{array}{lllll}0.4095785894628189 & 0.3201862567289394 & 0.2018860882934701\end{array}$

$\begin{array}{llll}0.4999187230115926 & 0.2763567504134071 & 0.1954877529885712\end{array}$

$\begin{array}{llll}0.7006284548864422 & 0.4247877455114336 & 0.2135629136426373\end{array}$

$\begin{array}{llll}0.7495977643777706 & 0.3409693994487238 & 0.2096452608319308\end{array}$

$\begin{array}{llll}0.6814648046035909 & 0.2728871469744058 & 0.2000955423181080\end{array}$

$\begin{array}{llll}0.5898662533105201 & 0.3206406775559514 & 0.2020564394921134\end{array}$

$\begin{array}{lllll}0.8217983047181975 & 0.6899888572795351 & 0.2246111896399281\end{array}$

$\begin{array}{llll}0.6858579897606465 & 0.8203801713390652 & 0.2285981401064049\end{array}$

$\begin{array}{llll}0.3102201591226974 & 0.8188309726592412 & 0.2291898609441669\end{array}$

$\begin{array}{llll}0.1750159656546038 & 0.6881501522744048 & 0.2246486851853998\end{array}$

$\begin{array}{llll}0.1743628496202371 & 0.3274812024009136 & 0.2133611057304669\end{array}$

$\begin{array}{lllll}0.3118341874837919 & 0.1991741614611137 & 0.2063279478111557\end{array}$

$\begin{array}{llll}0.6888605842716292 & 0.2006160118667326 & 0.2065936261394596\end{array}$

$\begin{array}{lllll}0.8248217110702879 & 0.3303218491753218 & 0.2140808815484221\end{array}$

$\begin{array}{llll}0.5000860725426970 & 0.2032039718879979 & 0.2016712171724519\end{array}$

$\begin{array}{llll}0.8198552907840806 & 0.5114248488636840 & 0.2203268622683059\end{array}$

$\begin{array}{llll}0.4979294801975083 & 0.8175471220643813 & 0.2287639660937570\end{array}$

$\begin{array}{llll}0.1776565656415824 & 0.5095419678451938 & 0.2197659561959937\end{array}$

\section{FeP-Pt(111) - Hfcc-45, vdW-DF-optPBE:}

Pt H C N Fe

1.00000000000000

$\begin{array}{lll}14.1349999999999998 & 0.0000000000000000 & 0.0000000000000000\end{array}$

$\begin{array}{llll}0.0000000000000000 & 14.6880000000000006 & 0.0000000000000000\end{array}$

$\begin{array}{llll}0.0000000000000000 & 0.0000000000000000 & 35.0000000000000000\end{array}$

$\mathrm{Pt} \quad \mathrm{H} \quad \mathrm{C} \quad \mathrm{N} \quad \mathrm{Fe}$

$\begin{array}{lllll}90 & 12 & 20 & 4 & 1\end{array}$

Direct

$\begin{array}{llll}0.6998999999999995 & 0.8333299999999966 & 0.0000000000000000\end{array}$

$\begin{array}{llll}0.7998899999999978 & 0.6666700000000034 & 0.0000000000000000\end{array}$

$\begin{array}{lll}0.5999199999999973 & 0.6666700000000034 & 0.0000000000000000\end{array}$

$\begin{array}{lllll}0.8998800000000031 & 0.8333299999999966 & 0.0000000000000000\end{array}$

$\begin{array}{lllll}0.2999599999999987 & 0.8333299999999966 & 0.0000000000000000\end{array}$

0.39994999999999690 .66667000000000340 .0000000000000000

$0.1999700000000004 \quad 0.66667000000000340 .0000000000000000$ 
$\begin{array}{lll}0.4999299999999991 & 0.8333299999999966 & 0.0000000000000000\end{array}$

$\begin{array}{llll}0.0000000000000000 & 0.6666700000000034 & 0.0000000000000000\end{array}$

$\begin{array}{llll}0.0999899999999982 & 0.8333299999999966 & 0.0000000000000000\end{array}$

$\begin{array}{llll}0.6998999999999995 & 0.1666600000000003 & 0.0000000000000000\end{array}$

$\begin{array}{llll}0.7998899999999978 & 0.0000000000000000 & 0.0000000000000000\end{array}$

$\begin{array}{llll}0.5999199999999973 & 0.0000000000000000 & 0.0000000000000000\end{array}$

$\begin{array}{lllll}0.8998800000000031 & 0.4999800000000008 & 0.0000000000000000\end{array}$

$\begin{array}{llll}0.8998800000000031 & 0.1666600000000003 & 0.0000000000000000\end{array}$

$\begin{array}{llll}0.7998899999999978 & 0.3333200000000005 & 0.0000000000000000\end{array}$

$\begin{array}{llll}0.2999599999999987 & 0.1666600000000003 & 0.0000000000000000\end{array}$

$\begin{array}{llll}0.3999499999999969 & 0.0000000000000000 & 0.0000000000000000\end{array}$

$\begin{array}{llll}0.1999700000000004 & 0.0000000000000000 & 0.0000000000000000\end{array}$

$\begin{array}{llll}0.4999299999999991 & 0.4999800000000008 & 0.0000000000000000\end{array}$

$\begin{array}{llll}0.4999299999999991 & 0.1666600000000003 & 0.0000000000000000\end{array}$

$\begin{array}{llll}0.5999199999999973 & 0.3333200000000005 & 0.0000000000000000\end{array}$

$\begin{array}{lllll}0.3999499999999969 & 0.3333200000000005 & 0.0000000000000000\end{array}$

$\begin{array}{llll}0.6998999999999995 & 0.4999800000000008 & 0.0000000000000000\end{array}$

$\begin{array}{llll}0.0000000000000000 & 0.0000000000000000 & 0.0000000000000000\end{array}$

$\begin{array}{llll}0.0999899999999982 & 0.4999800000000008 & 0.0000000000000000\end{array}$

$\begin{array}{llll}0.0999899999999982 & 0.1666600000000003 & 0.0000000000000000\end{array}$

$\begin{array}{llll}0.1999700000000004 & 0.3333200000000005 & 0.0000000000000000\end{array}$

$\begin{array}{lllll}0.0000000000000000 & 0.3333200000000005 & 0.0000000000000000\end{array}$

$\begin{array}{llll}0.2999599999999987 & 0.4999800000000008 & 0.0000000000000000\end{array}$

$\begin{array}{lllll}0.8998800000000031 & 0.7222200000000001 & 0.0659399999999977\end{array}$

$\begin{array}{llll}0.4999299999999991 & 0.7222200000000001 & 0.0659399999999977\end{array}$

$\begin{array}{lllll}0.6998999999999995 & 0.7222200000000001 & 0.0659399999999977\end{array}$

$\begin{array}{lllll}0.7998899999999978 & 0.8888800000000003 & 0.0659399999999977\end{array}$

$\begin{array}{llll}0.5999199999999973 & 0.8888800000000003 & 0.0659399999999977\end{array}$

$\begin{array}{lllll}0.0999899999999982 & 0.7222200000000001 & 0.0659399999999977\end{array}$

$\begin{array}{lllll}0.2999599999999987 & 0.7222200000000001 & 0.0659399999999977\end{array}$

$\begin{array}{lllll}0.3999499999999969 & 0.8888800000000003 & 0.0659399999999977\end{array}$

$\begin{array}{lllll}0.1999700000000004 & 0.8888800000000003 & 0.0659399999999977\end{array}$

$\begin{array}{llll}0.0000000000000000 & 0.8888800000000003 & 0.0659399999999977\end{array}$

$\begin{array}{lllll}0.8998800000000031 & 0.0555499999999967 & 0.0659399999999977\end{array}$

$\begin{array}{lllll}0.4999299999999991 & 0.0555499999999967 & 0.0659399999999977\end{array}$

$\begin{array}{lllll}0.6998999999999995 & 0.3888800000000003 & 0.0659399999999977\end{array}$

$\begin{array}{lllll}0.6998999999999995 & 0.0555499999999967 & 0.0659399999999977\end{array}$

$\begin{array}{lllll}0.7998899999999978 & 0.2222099999999969 & 0.0659399999999977\end{array}$

$\begin{array}{llll}0.5999199999999973 & 0.2222099999999969 & 0.0659399999999977\end{array}$

$\begin{array}{lllll}0.8998800000000031 & 0.3888800000000003 & 0.0659399999999977\end{array}$

$\begin{array}{lllll}0.7998899999999978 & 0.5555400000000006 & 0.0659399999999977\end{array}$

$\begin{array}{lllll}0.0999899999999982 & 0.0555499999999967 & 0.0659399999999977\end{array}$

$\begin{array}{llll}0.2999599999999987 & 0.3888800000000003 & 0.0659399999999977\end{array}$

$\begin{array}{lllll}0.2999599999999987 & 0.0555499999999967 & 0.0659399999999977\end{array}$

$\begin{array}{lllll}0.3999499999999969 & 0.2222099999999969 & 0.0659399999999977\end{array}$

$\begin{array}{lllll}0.1999700000000004 & 0.2222099999999969 & 0.0659399999999977\end{array}$

$\begin{array}{llll}0.4999299999999991 & 0.3888800000000003 & 0.0659399999999977\end{array}$ 
$\begin{array}{llll}0.5999199999999973 & 0.5555400000000006 & 0.0659399999999977\end{array}$ $\begin{array}{lllll}0.3999499999999969 & 0.5555400000000006 & 0.0659399999999977\end{array}$ $\begin{array}{llllll}0.0000000000000000 & 0.2222099999999969 & 0.0659399999999977\end{array}$ $\begin{array}{llll}0.0999899999999982 & 0.3888800000000003 & 0.0659399999999977\end{array}$ $\begin{array}{llll}0.1999700000000004 & 0.5555400000000006 & 0.0659399999999977\end{array}$ $\begin{array}{llll}0.0000000000000000 & 0.5555400000000006 & 0.0659399999999977\end{array}$ $\begin{array}{llll}0.9027259978472983 & 0.9470000667697533 & 0.1329981782833585\end{array}$ $\begin{array}{llll}0.8034337321045015 & 0.7838240058446146 & 0.1321314166450946\end{array}$ $\begin{array}{llll}0.5012690518439342 & 0.9491611422467585 & 0.1320438748283266\end{array}$ $\begin{array}{llll}0.5998682869227707 & 0.7850390020859805 & 0.1343200554226698\end{array}$ $\begin{array}{llll}0.4021314350020617 & 0.7853640611822712 & 0.1345188991305051\end{array}$ $\begin{array}{llll}0.7037312738240828 & 0.9486079119381614 & 0.1328383518810696\end{array}$ $\begin{array}{llll}0.0999663016453525 & 0.9467973919273049 & 0.1330050379346090\end{array}$ $\begin{array}{llll}0.1978114214066480 & 0.7835566567539374 & 0.1324410818551129\end{array}$ $\begin{array}{llll}0.0012736984677915 & 0.7813601093863056 & 0.1330634778332538\end{array}$ $\begin{array}{llll}0.2989273440047455 & 0.9478548671958293 & 0.1329312265234321\end{array}$ $\begin{array}{llll}0.9014392487269234 & 0.2772924124774718 & 0.1323074346350666\end{array}$ $\begin{array}{llll}0.8025451360275753 & 0.1121954779303209 & 0.1329677657404460\end{array}$ $\begin{array}{llll}0.5028636534121489 & 0.2730632171783185 & 0.1304797421245780\end{array}$ $\begin{array}{llll}0.6029759888491455 & 0.1105970724033796 & 0.1330327620293659\end{array}$ $\begin{array}{llll}0.4012541427346932 & 0.1106091845050301 & 0.1326930220123543\end{array}$ $\begin{array}{llll}0.7002670457290350 & 0.6200475438909692 & 0.1338978127835446\end{array}$ $\begin{array}{llll}0.7027730235248839 & 0.2775876318612163 & 0.1321214281050800\end{array}$ $\begin{array}{llll}0.8036881081959706 & 0.4495483248878287 & 0.1390187931522462\end{array}$ $\begin{array}{llll}0.6012286395877240 & 0.4396824931484389 & 0.1310872870931235\end{array}$ $\begin{array}{llll}0.9031071131044541 & 0.6163978106488486 & 0.1308422409954844\end{array}$ $\begin{array}{lllll}0.1002432066809291 & 0.2746369136634783 & 0.1318911358475887\end{array}$ $\begin{array}{llll}0.2013906947348829 & 0.1119125215723571 & 0.1326569252379031\end{array}$ $\begin{array}{lllll}0.0016895729843327 & 0.1117540823806564 & 0.1329395709458865\end{array}$ $\begin{array}{llll}0.3006326267609580 & 0.6209668778957749 & 0.1347318770396200\end{array}$ $\begin{array}{llll}0.3042426275416474 & 0.2773036007245864 & 0.1380122126195786\end{array}$ $\begin{array}{llll}0.3998505832888242 & 0.4417714376644781 & 0.1303245589073114\end{array}$ $\begin{array}{llll}0.1962381312145638 & 0.4418749494154923 & 0.1351475046978834\end{array}$ $\begin{array}{llll}0.5001809205739818 & 0.6046173385104225 & 0.1296900349274992\end{array}$ $\begin{array}{llll}0.9999906630615740 & 0.4438918535191760 & 0.1313377151406030\end{array}$ $\begin{array}{llll}0.1012757676313285 & 0.6141527345448168 & 0.1314749348407318\end{array}$ $\begin{array}{llll}0.2664878678993787 & 0.2552208195765999 & 0.2101390968280299\end{array}$ $\begin{array}{llll}0.2721840891879808 & 0.6938378192527637 & 0.2050985216081429\end{array}$ $\begin{array}{llll}0.7294164604920539 & 0.6880557908566445 & 0.2030352461211677\end{array}$ $\begin{array}{lllll}0.7195386543377111 & 0.2526431943240866 & 0.2236134263856044\end{array}$ $\begin{array}{lllll}0.8620476347985075 & 0.5562260237554213 & 0.2028436365273194\end{array}$ $\begin{array}{llll}0.8576437555593017 & 0.3718436859652456 & 0.2111907383455858\end{array}$ $\begin{array}{llll}0.5867174311924543 & 0.1288066681339899 & 0.2354295863484595\end{array}$ $\begin{array}{llll}0.3962882604420415 & 0.1263993197872111 & 0.2244492760689809\end{array}$ $\begin{array}{llll}0.1333965551956808 & 0.3841768211882055 & 0.2066286192562998\end{array}$ $\begin{array}{lllll}0.1365641919106650 & 0.5705706368790544 & 0.2093386255789156\end{array}$ $\begin{array}{llll}0.4096852686790697 & 0.8226105157597416 & 0.2087406496295614\end{array}$ 
$\begin{array}{llll}0.5934098494365045 & 0.8209947457183731 & 0.2086078294375966\end{array}$

$\begin{array}{llll}0.7000672611634968 & 0.3937254823560679 & 0.2139003842061555\end{array}$

$\begin{array}{llll}0.7975658733893951 & 0.4162744058677745 & 0.2060604518786988\end{array}$

$\begin{array}{llll}0.8005624405584584 & 0.5119826224109545 & 0.2003781871309869\end{array}$

$\begin{array}{llll}0.7028072012778779 & 0.5436248995023902 & 0.2046886879953931\end{array}$

$\begin{array}{llll}0.6660660754109529 & 0.3057651121499632 & 0.2206859321226204\end{array}$

$\begin{array}{llll}0.5714352073533675 & 0.2775307742449939 & 0.2229450922939478\end{array}$

$\begin{array}{llll}0.4161616828450221 & 0.2757520824006505 & 0.2133333824452137\end{array}$

$\begin{array}{llll}0.4437217727170761 & 0.1844664467092514 & 0.2231026225576116\end{array}$

$\begin{array}{llll}0.5404001078454286 & 0.1857372278958636 & 0.2289250414630573\end{array}$

$\begin{array}{llll}0.3216780624193518 & 0.3037298107980746 & 0.2019005770342659\end{array}$

$\begin{array}{lllll}0.2917984470303736 & 0.3981782372207761 & 0.2042353865387696\end{array}$

$\begin{array}{llll}0.2931203864756640 & 0.5492925814728906 & 0.2080742583396216\end{array}$

$\begin{array}{llll}0.1964150729292840 & 0.5243657250832428 & 0.2073046258505542\end{array}$

$\begin{array}{llll}0.1935519166338509 & 0.4284679625933450 & 0.2010327443632036\end{array}$

$\begin{array}{llll}0.3246988400515534 & 0.6407132142002752 & 0.1996681545083945\end{array}$

$\begin{array}{llll}0.4204235708674773 & 0.6732554058878222 & 0.1989631591624175\end{array}$

$\begin{array}{llll}0.5798225328231936 & 0.6716999581825931 & 0.1980362082111995\end{array}$

$\begin{array}{llll}0.6753394429440576 & 0.6367048221442673 & 0.1969749335966497\end{array}$

$\begin{array}{llll}0.5540163993201190 & 0.7677775761142485 & 0.1936515780784234\end{array}$

$\begin{array}{llll}0.4480014480978198 & 0.7686645856308800 & 0.1938022713053584\end{array}$

$\begin{array}{llll}0.6442854166570910 & 0.4722852017326673 & 0.2117106228549943\end{array}$

$\begin{array}{llll}0.4936775483832250 & 0.3322629896151881 & 0.2141707409591334\end{array}$

$\begin{array}{llll}0.4996873110026243 & 0.6166311903687571 & 0.1985388934908698\end{array}$

$\begin{array}{llll}0.3502329447339747 & 0.4718271408173536 & 0.2079164269718987\end{array}$

$\begin{array}{llll}0.4983504037966426 & 0.4656059391619536 & 0.1945074609032957\end{array}$

\section{FeP-Pt(111) - B-0, vdW-DF-optPBE:}

Pt H C N Fe

1.00000000000000

$\begin{array}{llll}14.1349999999999998 & 0.0000000000000000 & 0.0000000000000000\end{array}$

$\begin{array}{lll}0.0000000000000000 & 14.6880000000000006 & 0.0000000000000000\end{array}$

$\begin{array}{llll}0.0000000000000000 & 0.0000000000000000 & 35.0000000000000000\end{array}$

$\mathrm{Pt} \quad \mathrm{H} \quad \mathrm{C} \quad \mathrm{N} \quad \mathrm{Fe}$

$\begin{array}{lllll}90 & 12 & 20 & 4 & 1\end{array}$

Direct

0.69989999999999950 .83332999999999660 .0000000000000000

$\begin{array}{llll}0.7998899999999978 & 0.6666700000000034 & 0.0000000000000000\end{array}$

$\begin{array}{llll}0.5999199999999973 & 0.6666700000000034 & 0.0000000000000000\end{array}$

$\begin{array}{lllll}0.8998800000000031 & 0.8333299999999966 & 0.0000000000000000\end{array}$

$\begin{array}{lllll}0.2999599999999987 & 0.8333299999999966 & 0.0000000000000000\end{array}$

$\begin{array}{lll}0.3999499999999969 & 0.6666700000000034 & 0.0000000000000000\end{array}$

0.19997000000000040 .66667000000000340 .0000000000000000

$\begin{array}{llll}0.4999299999999991 & 0.8333299999999966 & 0.0000000000000000\end{array}$

$\begin{array}{lllll}0.0000000000000000 & 0.6666700000000034 & 0.0000000000000000\end{array}$

$\begin{array}{lllll}0.0999899999999982 & 0.8333299999999966 & 0.0000000000000000\end{array}$

$\begin{array}{llll}0.6998999999999995 & 0.1666600000000003 & 0.0000000000000000\end{array}$ 
$\begin{array}{lll}0.7998899999999978 & 0.0000000000000000 & 0.0000000000000000\end{array}$

$\begin{array}{llll}0.5999199999999973 & 0.0000000000000000 & 0.0000000000000000\end{array}$

$\begin{array}{llll}0.8998800000000031 & 0.4999800000000008 & 0.0000000000000000\end{array}$

$\begin{array}{llll}0.8998800000000031 & 0.1666600000000003 & 0.0000000000000000\end{array}$

$\begin{array}{llll}0.7998899999999978 & 0.3333200000000005 & 0.0000000000000000\end{array}$

$\begin{array}{llll}0.2999599999999987 & 0.1666600000000003 & 0.0000000000000000\end{array}$

$\begin{array}{lllll}0.3999499999999969 & 0.0000000000000000 & 0.0000000000000000\end{array}$

$\begin{array}{lll}0.1999700000000004 & 0.0000000000000000 & 0.0000000000000000\end{array}$

$\begin{array}{llll}0.4999299999999991 & 0.4999800000000008 & 0.0000000000000000\end{array}$

$\begin{array}{llll}0.4999299999999991 & 0.1666600000000003 & 0.0000000000000000\end{array}$

$\begin{array}{llll}0.5999199999999973 & 0.3333200000000005 & 0.0000000000000000\end{array}$

$\begin{array}{llll}0.3999499999999969 & 0.3333200000000005 & 0.0000000000000000\end{array}$

$\begin{array}{llll}0.6998999999999995 & 0.4999800000000008 & 0.0000000000000000\end{array}$

$\begin{array}{llll}0.0000000000000000 & 0.0000000000000000 & 0.0000000000000000\end{array}$

$\begin{array}{llll}0.0999899999999982 & 0.4999800000000008 & 0.0000000000000000\end{array}$

$\begin{array}{llll}0.0999899999999982 & 0.1666600000000003 & 0.0000000000000000\end{array}$

$\begin{array}{lllll}0.1999700000000004 & 0.3333200000000005 & 0.0000000000000000\end{array}$

$\begin{array}{lllll}0.0000000000000000 & 0.3333200000000005 & 0.0000000000000000\end{array}$

$\begin{array}{llll}0.2999599999999987 & 0.4999800000000008 & 0.0000000000000000\end{array}$

$\begin{array}{llll}0.8998800000000031 & 0.7222200000000001 & 0.0659399999999977\end{array}$

$\begin{array}{lllll}0.4999299999999991 & 0.7222200000000001 & 0.0659399999999977\end{array}$

$\begin{array}{lllll}0.6998999999999995 & 0.7222200000000001 & 0.0659399999999977\end{array}$

$\begin{array}{lllll}0.7998899999999978 & 0.8888800000000003 & 0.0659399999999977\end{array}$

$\begin{array}{llll}0.5999199999999973 & 0.8888800000000003 & 0.0659399999999977\end{array}$

$\begin{array}{lllll}0.0999899999999982 & 0.7222200000000001 & 0.0659399999999977\end{array}$

$\begin{array}{lllll}0.2999599999999987 & 0.7222200000000001 & 0.0659399999999977\end{array}$

$\begin{array}{lllll}0.3999499999999969 & 0.8888800000000003 & 0.0659399999999977\end{array}$

$\begin{array}{lllll}0.1999700000000004 & 0.8888800000000003 & 0.0659399999999977\end{array}$

$\begin{array}{llll}0.0000000000000000 & 0.8888800000000003 & 0.0659399999999977\end{array}$

$\begin{array}{lllll}0.8998800000000031 & 0.0555499999999967 & 0.0659399999999977\end{array}$

$\begin{array}{lllll}0.4999299999999991 & 0.0555499999999967 & 0.0659399999999977\end{array}$

$\begin{array}{lllll}0.6998999999999995 & 0.3888800000000003 & 0.0659399999999977\end{array}$

$\begin{array}{lllll}0.6998999999999995 & 0.0555499999999967 & 0.0659399999999977\end{array}$

$\begin{array}{llll}0.7998899999999978 & 0.2222099999999969 & 0.0659399999999977\end{array}$

$\begin{array}{lllll}0.5999199999999973 & 0.2222099999999969 & 0.0659399999999977\end{array}$

$\begin{array}{lllll}0.8998800000000031 & 0.3888800000000003 & 0.0659399999999977\end{array}$

$\begin{array}{lllll}0.7998899999999978 & 0.5555400000000006 & 0.0659399999999977\end{array}$

$\begin{array}{llll}0.0999899999999982 & 0.0555499999999967 & 0.0659399999999977\end{array}$

$\begin{array}{llll}0.2999599999999987 & 0.3888800000000003 & 0.0659399999999977\end{array}$

$\begin{array}{lllll}0.2999599999999987 & 0.0555499999999967 & 0.0659399999999977\end{array}$

$\begin{array}{lllll}0.3999499999999969 & 0.2222099999999969 & 0.0659399999999977\end{array}$

$\begin{array}{lllll}0.1999700000000004 & 0.2222099999999969 & 0.0659399999999977\end{array}$

$\begin{array}{llll}0.4999299999999991 & 0.3888800000000003 & 0.0659399999999977\end{array}$

$\begin{array}{lllll}0.5999199999999973 & 0.5555400000000006 & 0.0659399999999977\end{array}$

$\begin{array}{lllll}0.3999499999999969 & 0.5555400000000006 & 0.0659399999999977\end{array}$

$\begin{array}{lllll}0.0000000000000000 & 0.2222099999999969 & 0.0659399999999977\end{array}$

$\begin{array}{llll}0.0999899999999982 & 0.3888800000000003 & 0.0659399999999977\end{array}$ 
$0.1999700000000004 \quad 0.5555400000000006 \quad 0.0659399999999977$ $\begin{array}{lllll}0.0000000000000000 & 0.5555400000000006 & 0.0659399999999977\end{array}$ $\begin{array}{llll}0.9003648989181875 & 0.9440680355924086 & 0.1332178385976732\end{array}$ $\begin{array}{llll}0.8025023884246349 & 0.7816997343878223 & 0.1320928725175934\end{array}$ $\begin{array}{llll}0.4998372271843629 & 0.9435906265472551 & 0.1333227001302738\end{array}$ $\begin{array}{llll}0.6004674662409191 & 0.7797874540781168 & 0.1324257367379611\end{array}$ $\begin{array}{llll}0.3990039899612171 & 0.7798493332512462 & 0.1323914251066929\end{array}$ $\begin{array}{llll}0.6996167971609438 & 0.9437366161278897 & 0.1330837376832808\end{array}$ $\begin{array}{llll}0.0989156651514700 & 0.9441127793556213 & 0.1331603332627372\end{array}$ $\begin{array}{llll}0.1969815833572961 & 0.7818022483182689 & 0.1320263796123695\end{array}$ $\begin{array}{llll}0.9995482663753820 & 0.7796929524218564 & 0.1329606655389135\end{array}$ $\begin{array}{llll}0.2999155933222205 & 0.9436718934269048 & 0.1331825103953470\end{array}$ $\begin{array}{llll}0.9027274532434418 & 0.2778338111834208 & 0.1311449049811141\end{array}$ $\begin{array}{llll}0.8023830815063073 & 0.1064594153752888 & 0.1319900312507508\end{array}$ $\begin{array}{llll}0.4998341760863428 & 0.2735803707871287 & 0.1304475933729724\end{array}$ $\begin{array}{llll}0.5995553882791439 & 0.1078769450099535 & 0.1323152749709198\end{array}$ $\begin{array}{llll}0.3996806973476046 & 0.1078786449229779 & 0.1323144440865262\end{array}$ $\begin{array}{llll}0.7064415987423108 & 0.6172840604634329 & 0.1356521041530718\end{array}$ $\begin{array}{llll}0.7054302600008865 & 0.2722958712031844 & 0.1358018415256323\end{array}$ $\begin{array}{llll}0.7927428607083773 & 0.4453475525312039 & 0.1365599006353074\end{array}$ $\begin{array}{llll}0.5957899013224036 & 0.4436363171553306 & 0.1307650324021312\end{array}$ $\begin{array}{llll}0.9030258433315118 & 0.6111682392036105 & 0.1314548494784080\end{array}$ $\begin{array}{llll}0.0966986306040761 & 0.2779515520441936 & 0.1311732758162001\end{array}$ $\begin{array}{llll}0.1974217278197149 & 0.1064543373049863 & 0.1319658519735789\end{array}$ $\begin{array}{llll}0.9998856124512151 & 0.1091695908360241 & 0.1329462975518553\end{array}$ $\begin{array}{lllll}0.2923607012998709 & 0.6170765081193264 & 0.1356314722581048\end{array}$ $\begin{array}{llll}0.2941255549825737 & 0.2722120838387977 & 0.1358741073623732\end{array}$ $\begin{array}{llll}0.4035233477463555 & 0.4434361825123245 & 0.1309405715541412\end{array}$ $\begin{array}{llll}0.2059031050648590 & 0.4446640463337029 & 0.1362607084326393\end{array}$ $\begin{array}{llll}0.4991558296831891 & 0.6139003208814979 & 0.1300069242951103\end{array}$ $\begin{array}{llll}0.9992905141596804 & 0.4443616476032305 & 0.1321819625276300\end{array}$ $\begin{array}{llll}0.0960859672897850 & 0.6113248662459244 & 0.1314553647557488\end{array}$ $\begin{array}{llll}0.4999444873709779 & 0.1406793655069777 & 0.2249553324343373\end{array}$ $\begin{array}{llll}0.1801278201694746 & 0.4455630577196996 & 0.2076587339469315\end{array}$ $\begin{array}{llll}0.4983559380948141 & 0.7507856896488269 & 0.2257235545769941\end{array}$ $\begin{array}{llll}0.8182644299243895 & 0.4471589197374836 & 0.2078762379500495\end{array}$ $\begin{array}{llll}0.6803702015734427 & 0.7591138191348978 & 0.2145343064437634\end{array}$ $\begin{array}{llll}0.8164006766977021 & 0.6324062161145488 & 0.2007721598276681\end{array}$ $\begin{array}{llll}0.8176635275481797 & 0.2606594183758199 & 0.2006133327784147\end{array}$ $\begin{array}{llll}0.6818742975353332 & 0.1336332801746770 & 0.2139813404845326\end{array}$ $\begin{array}{llll}0.3182166333289843 & 0.1330859834586122 & 0.2142097592320039\end{array}$ $\begin{array}{llll}0.1817345374223939 & 0.2592649139401313 & 0.2007265669497882\end{array}$ $\begin{array}{llll}0.1809115563841017 & 0.6312583592640670 & 0.2005006813321219\end{array}$ $\begin{array}{llll}0.3165463102552920 & 0.7583329086391060 & 0.2142917624719061\end{array}$ $\begin{array}{llll}0.6950562726285487 & 0.5325197354568473 & 0.2044361604992982\end{array}$ $\begin{array}{llll}0.7401211347914971 & 0.6217396746295610 & 0.2001529284362944\end{array}$ $\begin{array}{llll}0.6711749751943755 & 0.6859641324872794 & 0.2119647016730795\end{array}$ 
$\begin{array}{llll}0.5871124302873283 & 0.6362628553499403 & 0.2194254864574111\end{array}$

$\begin{array}{lllll}0.7449252054331799 & 0.4466399677689311 & 0.1972597815884869\end{array}$

$\begin{array}{llll}0.6960436056012494 & 0.3603838663448298 & 0.2044722426096660\end{array}$

$\begin{array}{llll}0.5882764215017376 & 0.2561043900289220 & 0.2192853642924553\end{array}$

$\begin{array}{llll}0.6725340839916549 & 0.2067834859668345 & 0.2116649225403848\end{array}$

$\begin{array}{llll}0.7414299485820973 & 0.2712740849475050 & 0.2001055741947566\end{array}$

$\begin{array}{llll}0.4998503817419847 & 0.2149238708728189 & 0.2236779183581475\end{array}$

$\begin{array}{llll}0.4112560578560783 & 0.2558698387777165 & 0.2194612915251400\end{array}$

$\begin{array}{llll}0.3029506061949281 & 0.3596403023202244 & 0.2045523992505789\end{array}$

$\begin{array}{llll}0.2579360292878075 & 0.2703872591126627 & 0.2001700876924838\end{array}$

$\begin{array}{llll}0.3272301221339595 & 0.2062659402421237 & 0.2117297345220118\end{array}$

$\begin{array}{lllll}0.2535221070064066 & 0.4455111741403362 & 0.1971402189073542\end{array}$

$\begin{array}{lllll}0.3025585041387571 & 0.5317457174630770 & 0.2041551336138482\end{array}$

$\begin{array}{llll}0.4100807421454476 & 0.6357389057575338 & 0.2194058851840540\end{array}$

$\begin{array}{llll}0.4985083071508392 & 0.6766033014990516 & 0.2239929428315604\end{array}$

$\begin{array}{llll}0.3260251703561860 & 0.6851920924068186 & 0.2116350076785660\end{array}$

$\begin{array}{llll}0.2571693291542486 & 0.6207514607838048 & 0.1997847918955973\end{array}$

$\begin{array}{llll}0.6031636440477579 & 0.5423310587900022 & 0.2147114092542386\end{array}$

$\begin{array}{llll}0.6041839503135389 & 0.3501053630006145 & 0.2148268154452475\end{array}$

$\begin{array}{llll}0.3943271228253704 & 0.5418236807266805 & 0.2146738238635006\end{array}$

$\begin{array}{llll}0.3948427564651467 & 0.3498086612568823 & 0.2149932740460336\end{array}$

$\begin{array}{llll}0.4990504920387982 & 0.4459568885052891 & 0.1982437645244625\end{array}$

\section{FeP-Pt(111) - B-45, High Spin, vdW-DF-optPBE:}

$\mathrm{Pt} \mathrm{H} \mathrm{C} \mathrm{N} \mathrm{Fe}$

1.00000000000000

$\begin{array}{llll}14.1349999999999998 & 0.0000000000000000 & 0.0000000000000000\end{array}$

$\begin{array}{lll}0.0000000000000000 & 14.6880000000000006 & 0.0000000000000000\end{array}$

$\begin{array}{llll}0.0000000000000000 & 0.0000000000000000 & 35.0000000000000000\end{array}$

$\mathrm{Pt} \quad \mathrm{H} \quad \mathrm{C} \quad \mathrm{N} \quad \mathrm{Fe}$

$\begin{array}{lllll}90 & 12 & 20 & 4 & 1\end{array}$

Direct

0.69989999999999950 .83332999999999660 .0000000000000000

$\begin{array}{llll}0.7998899999999978 & 0.6666700000000034 & 0.0000000000000000\end{array}$

$\begin{array}{lllll}0.5999199999999973 & 0.6666700000000034 & 0.0000000000000000\end{array}$

$\begin{array}{lllll}0.8998800000000031 & 0.8333299999999966 & 0.0000000000000000\end{array}$

$\begin{array}{llll}0.2999599999999987 & 0.8333299999999966 & 0.0000000000000000\end{array}$

$\begin{array}{lll}0.3999499999999969 & 0.6666700000000034 & 0.0000000000000000\end{array}$

0.19997000000000040 .66667000000000340 .0000000000000000

$\begin{array}{llll}0.4999299999999991 & 0.8333299999999966 & 0.0000000000000000\end{array}$

$\begin{array}{lllll}0.0000000000000000 & 0.6666700000000034 & 0.0000000000000000\end{array}$

$\begin{array}{llll}0.0999899999999982 & 0.8333299999999966 & 0.0000000000000000\end{array}$

$\begin{array}{lll}0.6998999999999995 & 0.1666600000000003 & 0.0000000000000000\end{array}$

$\begin{array}{llll}0.7998899999999978 & 0.0000000000000000 & 0.0000000000000000\end{array}$

$\begin{array}{llll}0.5999199999999973 & 0.0000000000000000 & 0.0000000000000000\end{array}$

$\begin{array}{llll}0.8998800000000031 & 0.4999800000000008 & 0.0000000000000000\end{array}$

$\begin{array}{llll}0.8998800000000031 & 0.1666600000000003 & 0.0000000000000000\end{array}$ 
$0.7998899999999978 \quad 0.33332000000000050 .0000000000000000$ $\begin{array}{llll}0.2999599999999987 & 0.1666600000000003 & 0.0000000000000000\end{array}$ $\begin{array}{llll}0.3999499999999969 & 0.0000000000000000 & 0.0000000000000000\end{array}$ $\begin{array}{lll}0.1999700000000004 & 0.0000000000000000 & 0.0000000000000000\end{array}$ $\begin{array}{llll}0.4999299999999991 & 0.4999800000000008 & 0.0000000000000000\end{array}$ $\begin{array}{llll}0.4999299999999991 & 0.1666600000000003 & 0.0000000000000000\end{array}$ $\begin{array}{llll}0.5999199999999973 & 0.3333200000000005 & 0.0000000000000000\end{array}$ $\begin{array}{llll}0.3999499999999969 & 0.3333200000000005 & 0.0000000000000000\end{array}$ $\begin{array}{llll}0.6998999999999995 & 0.4999800000000008 & 0.0000000000000000\end{array}$ $\begin{array}{llll}0.0000000000000000 & 0.0000000000000000 & 0.0000000000000000\end{array}$ $\begin{array}{llll}0.0999899999999982 & 0.4999800000000008 & 0.0000000000000000\end{array}$ $\begin{array}{llll}0.0999899999999982 & 0.1666600000000003 & 0.0000000000000000\end{array}$ $\begin{array}{llll}0.1999700000000004 & 0.3333200000000005 & 0.0000000000000000\end{array}$ $\begin{array}{llll}0.0000000000000000 & 0.3333200000000005 & 0.0000000000000000\end{array}$ $\begin{array}{llll}0.2999599999999987 & 0.4999800000000008 & 0.0000000000000000\end{array}$ $\begin{array}{llll}0.8998800000000031 & 0.7222200000000001 & 0.0659399999999977\end{array}$ $\begin{array}{lllll}0.4999299999999991 & 0.7222200000000001 & 0.0659399999999977\end{array}$ $\begin{array}{lllll}0.6998999999999995 & 0.7222200000000001 & 0.0659399999999977\end{array}$ $\begin{array}{llll}0.7998899999999978 & 0.8888800000000003 & 0.0659399999999977\end{array}$ $\begin{array}{llll}0.5999199999999973 & 0.8888800000000003 & 0.0659399999999977\end{array}$ $\begin{array}{lllll}0.0999899999999982 & 0.7222200000000001 & 0.0659399999999977\end{array}$ $\begin{array}{lllll}0.2999599999999987 & 0.7222200000000001 & 0.0659399999999977\end{array}$ $\begin{array}{llll}0.3999499999999969 & 0.8888800000000003 & 0.0659399999999977\end{array}$ $\begin{array}{llll}0.1999700000000004 & 0.8888800000000003 & 0.0659399999999977\end{array}$ $\begin{array}{llll}0.0000000000000000 & 0.8888800000000003 & 0.0659399999999977\end{array}$ $\begin{array}{lllll}0.8998800000000031 & 0.0555499999999967 & 0.0659399999999977\end{array}$ $\begin{array}{lllll}0.4999299999999991 & 0.0555499999999967 & 0.0659399999999977\end{array}$ $\begin{array}{llll}0.6998999999999995 & 0.3888800000000003 & 0.0659399999999977\end{array}$ $\begin{array}{llll}0.6998999999999995 & 0.0555499999999967 & 0.0659399999999977\end{array}$ $\begin{array}{lllll}0.7998899999999978 & 0.2222099999999969 & 0.0659399999999977\end{array}$ $\begin{array}{lllll}0.5999199999999973 & 0.2222099999999969 & 0.0659399999999977\end{array}$ $\begin{array}{lllll}0.8998800000000031 & 0.3888800000000003 & 0.0659399999999977\end{array}$ $\begin{array}{lllll}0.7998899999999978 & 0.5555400000000006 & 0.0659399999999977\end{array}$ $\begin{array}{lllll}0.0999899999999982 & 0.0555499999999967 & 0.0659399999999977\end{array}$ $\begin{array}{lllll}0.2999599999999987 & 0.3888800000000003 & 0.0659399999999977\end{array}$ $\begin{array}{lllll}0.2999599999999987 & 0.0555499999999967 & 0.0659399999999977\end{array}$ $\begin{array}{lllll}0.3999499999999969 & 0.2222099999999969 & 0.0659399999999977\end{array}$ $\begin{array}{lllll}0.1999700000000004 & 0.2222099999999969 & 0.0659399999999977\end{array}$ $\begin{array}{llll}0.4999299999999991 & 0.3888800000000003 & 0.0659399999999977\end{array}$ $\begin{array}{lllll}0.5999199999999973 & 0.5555400000000006 & 0.0659399999999977\end{array}$ $\begin{array}{llll}0.3999499999999969 & 0.5555400000000006 & 0.0659399999999977\end{array}$ $\begin{array}{llll}0.0000000000000000 & 0.2222099999999969 & 0.0659399999999977\end{array}$ $\begin{array}{llll}0.0999899999999982 & 0.3888800000000003 & 0.0659399999999977\end{array}$ $\begin{array}{llll}0.1999700000000004 & 0.5555400000000006 & 0.0659399999999977\end{array}$ $\begin{array}{lllll}0.0000000000000000 & 0.5555400000000006 & 0.0659399999999977\end{array}$ $\begin{array}{llll}0.9009757785786405 & 0.9446048214012137 & 0.1330073279047710\end{array}$ $\begin{array}{llll}0.8018167582329312 & 0.7811597471299478 & 0.1325382907103858\end{array}$ 
$\begin{array}{llll}0.5002109111956372 & 0.9441389746829429 & 0.1316387930878733\end{array}$ $\begin{array}{llll}0.5990821786261336 & 0.7790110428193202 & 0.1361254810742381\end{array}$ $\begin{array}{lllll}0.4013885010169176 & 0.7789126463280809 & 0.1360565318142761\end{array}$ $\begin{array}{llll}0.7012803504739082 & 0.9443103320927975 & 0.1326755118584799\end{array}$ $\begin{array}{llll}0.0989584677036959 & 0.9447165130925512 & 0.1330100733607082\end{array}$ $\begin{array}{llll}0.1980229499292250 & 0.7812128034574997 & 0.1324534695508959\end{array}$ $\begin{array}{llll}0.9997512151814831 & 0.7802019115013579 & 0.1330838346824947\end{array}$ $\begin{array}{llll}0.2989409963063281 & 0.9444343414847651 & 0.1325967986067411\end{array}$ $\begin{array}{llll}0.9012355216931525 & 0.2743933712152611 & 0.1318726930377068\end{array}$ $\begin{array}{llll}0.8014022803687979 & 0.1082007009407349 & 0.1322228216701404\end{array}$ $\begin{array}{llll}0.4990487567085751 & 0.2806189528675702 & 0.1287707176963053\end{array}$ $\begin{array}{llll}0.5996738166054669 & 0.1092700775485440 & 0.1364821329703716\end{array}$ $\begin{array}{llll}0.4018082646170953 & 0.1090299694735535 & 0.1365840804661858\end{array}$ $\begin{array}{llll}0.7010864702929567 & 0.6156978079989983 & 0.1343899073737873\end{array}$ $\begin{array}{llll}0.7009049188934497 & 0.2737357989905362 & 0.1343957531219431\end{array}$ $\begin{array}{lllll}0.8063548409358958 & 0.4484209218002775 & 0.1368902369720928\end{array}$ $\begin{array}{llll}0.6038572192902905 & 0.4429887792965843 & 0.1297161154012727\end{array}$ $\begin{array}{llll}0.9013958518124241 & 0.6157488215899392 & 0.1316164972929315\end{array}$ $\begin{array}{llll}0.0996288805880923 & 0.2748310593838355 & 0.1319014175883879\end{array}$ $\begin{array}{llll}0.1987286227646692 & 0.1081328001430819 & 0.1322365993461005\end{array}$ $\begin{array}{llll}0.0000228450059652 & 0.1092689249858694 & 0.1328872013820686\end{array}$ $\begin{array}{llll}0.2985423724438903 & 0.6159962482434196 & 0.1343215087104426\end{array}$ $\begin{array}{llll}0.2987752138900746 & 0.2728929540278102 & 0.1347532615703457\end{array}$ $\begin{array}{llll}0.3949577605426953 & 0.4426719752229999 & 0.1297287099771367\end{array}$ $\begin{array}{llll}0.1936484748230356 & 0.4500277071956660 & 0.1363007149393454\end{array}$ $\begin{array}{llll}0.5002391521118597 & 0.6043430482441750 & 0.1293970246543168\end{array}$ $\begin{array}{lllll}0.0000405371528061 & 0.4435232022320719 & 0.1315590313555788\end{array}$ $\begin{array}{llll}0.0971756588403707 & 0.6163303654789587 & 0.1318532348369672\end{array}$ $\begin{array}{llll}0.2730130376472530 & 0.2217441838820946 & 0.2068305701984059\end{array}$ $\begin{array}{llll}0.2712571485970940 & 0.6619022862283046 & 0.2056334141388945\end{array}$ $\begin{array}{llll}0.7295048540548095 & 0.6635846125651383 & 0.2055917934757758\end{array}$ $\begin{array}{llll}0.7301859109191113 & 0.2233869631687924 & 0.2063372018112943\end{array}$ $\begin{array}{llll}0.8633613518830145 & 0.5351502243396169 & 0.2061328692091635\end{array}$ $\begin{array}{llll}0.8626595828211521 & 0.3491951960151383 & 0.2079935718601078\end{array}$ $\begin{array}{llll}0.5928435583752432 & 0.0901747128766885 & 0.2111089964982753\end{array}$ $\begin{array}{llll}0.4110854397624237 & 0.0896087950997853 & 0.2110413030071072\end{array}$ $\begin{array}{llll}0.1403290137938527 & 0.3470715001167903 & 0.2089640796007188\end{array}$ $\begin{array}{llll}0.1392281657907120 & 0.5329620533377479 & 0.2056290897679034\end{array}$ $\begin{array}{llll}0.4088211502054619 & 0.7957770060521483 & 0.2109753265735108\end{array}$ $\begin{array}{llll}0.5913718608673122 & 0.7961725782216007 & 0.2110520058744001\end{array}$ $\begin{array}{lllll}0.7047704263567738 & 0.3671026262522474 & 0.2077706183933898\end{array}$ $\begin{array}{llll}0.8018752342722451 & 0.3938952238770241 & 0.2051489929857340\end{array}$ $\begin{array}{llll}0.8028309712717530 & 0.4904552195343612 & 0.2020835262251737\end{array}$ $\begin{array}{llll}0.7045853666765467 & 0.5192920705686674 & 0.2051354186157113\end{array}$ $\begin{array}{llll}0.6755836263812469 & 0.2743698417861182 & 0.2005454692217725\end{array}$ $\begin{array}{llll}0.5809675732760766 & 0.2387809573157682 & 0.2005125493304121\end{array}$ $\begin{array}{llll}0.4219978006268761 & 0.2381519787820210 & 0.2002387957246512\end{array}$ 
$\begin{array}{llll}0.4484551713127800 & 0.1415524652680062 & 0.1944226495330881\end{array}$

$\begin{array}{llll}0.5553139802967166 & 0.1419231112149362 & 0.1944417897034327\end{array}$

$\begin{array}{llll}0.3269252892303811 & 0.2730195721274313 & 0.2005631969889663\end{array}$

$\begin{array}{lllll}0.2975395382477615 & 0.3656326904378560 & 0.2082063630160249\end{array}$

$\begin{array}{llll}0.2976955434014030 & 0.5177417336568837 & 0.2039214288305473\end{array}$

$\begin{array}{llll}0.1994018484639497 & 0.4880195960447864 & 0.2010407392318356\end{array}$

$\begin{array}{lllll}0.2010583238903578 & 0.3920022395178151 & 0.2063622958525357\end{array}$

$\begin{array}{llll}0.3253838240119293 & 0.6119561636953748 & 0.1978032907925140\end{array}$

$\begin{array}{llll}0.4208126001686026 & 0.6477017704980952 & 0.1986234531096756\end{array}$

$\begin{array}{llll}0.5801069021262819 & 0.6479785416111312 & 0.1989534925458472\end{array}$

$\begin{array}{llll}0.6756423808114604 & 0.6127827151140437 & 0.1984416296814473\end{array}$

$\begin{array}{llll}0.5536732929295454 & 0.7448589591855495 & 0.1942352958838995\end{array}$

$\begin{array}{llll}0.4468926861089528 & 0.7447823688858364 & 0.1941080001421795\end{array}$

$\begin{array}{llll}0.6470179951560340 & 0.4441268173488453 & 0.2080593008992437\end{array}$

$\begin{array}{llll}0.5012889954372568 & 0.2929722472890006 & 0.2023027022678647\end{array}$

$\begin{array}{lllll}0.5004537559170856 & 0.5928594743269002 & 0.2001421586883367\end{array}$

$\begin{array}{lllll}0.3556406605677296 & 0.4431913537283307 & 0.2072832440748158\end{array}$

$\begin{array}{llll}0.5017455123745620 & 0.4408977180222138 & 0.1944623886291197\end{array}$

FeP-Pt(111) - B-45, Intermediate Spin, vdW-DF-optPBE:

$\mathrm{Pt} \mathrm{H} \mathrm{C} \mathrm{N} \mathrm{Fe}$

1.00000000000000

$\begin{array}{lll}14.1349999999999998 & 0.0000000000000000 & 0.0000000000000000\end{array}$

$\begin{array}{lll}0.0000000000000000 & 14.6880000000000006 & 0.0000000000000000\end{array}$

$\begin{array}{llll}0.0000000000000000 & 0.0000000000000000 & 35.0000000000000000\end{array}$

$\mathrm{Pt} \quad \mathrm{H} \quad \mathrm{C} \quad \mathrm{N} \quad \mathrm{Fe}$

$\begin{array}{lllll}90 & 12 & 20 & 4 & 1\end{array}$

Direct

0.69989999999999950 .83332999999999660 .0000000000000000

$\begin{array}{llll}0.7998899999999978 & 0.6666700000000034 & 0.0000000000000000\end{array}$

$\begin{array}{lllll}0.5999199999999973 & 0.6666700000000034 & 0.0000000000000000\end{array}$

$\begin{array}{llll}0.8998800000000031 & 0.8333299999999966 & 0.0000000000000000\end{array}$

$\begin{array}{llll}0.2999599999999987 & 0.8333299999999966 & 0.0000000000000000\end{array}$

$\begin{array}{lll}0.3999499999999969 & 0.6666700000000034 & 0.0000000000000000\end{array}$

0.19997000000000040 .66667000000000340 .0000000000000000

$\begin{array}{lllll}0.4999299999999991 & 0.8333299999999966 & 0.0000000000000000\end{array}$

$\begin{array}{llll}0.0000000000000000 & 0.6666700000000034 & 0.0000000000000000\end{array}$

$\begin{array}{llll}0.0999899999999982 & 0.8333299999999966 & 0.0000000000000000\end{array}$

$\begin{array}{llll}0.6998999999999995 & 0.1666600000000003 & 0.0000000000000000\end{array}$

$\begin{array}{llll}0.7998899999999978 & 0.0000000000000000 & 0.0000000000000000\end{array}$

$\begin{array}{lllll}0.5999199999999973 & 0.0000000000000000 & 0.0000000000000000\end{array}$

$\begin{array}{llll}0.8998800000000031 & 0.4999800000000008 & 0.0000000000000000\end{array}$

$\begin{array}{llll}0.8998800000000031 & 0.1666600000000003 & 0.0000000000000000\end{array}$

$\begin{array}{llll}0.7998899999999978 & 0.3333200000000005 & 0.0000000000000000\end{array}$

$\begin{array}{lllll}0.2999599999999987 & 0.1666600000000003 & 0.0000000000000000\end{array}$

$\begin{array}{lllll}0.3999499999999969 & 0.0000000000000000 & 0.0000000000000000\end{array}$

$\begin{array}{lllll}0.1999700000000004 & 0.0000000000000000 & 0.0000000000000000\end{array}$ 
$\begin{array}{llll}0.4999299999999991 & 0.4999800000000008 & 0.0000000000000000\end{array}$

$\begin{array}{llll}0.4999299999999991 & 0.1666600000000003 & 0.0000000000000000\end{array}$

$\begin{array}{llll}0.5999199999999973 & 0.3333200000000005 & 0.0000000000000000\end{array}$

$\begin{array}{llll}0.3999499999999969 & 0.3333200000000005 & 0.0000000000000000\end{array}$

$\begin{array}{llll}0.6998999999999995 & 0.4999800000000008 & 0.0000000000000000\end{array}$

$\begin{array}{llll}0.0000000000000000 & 0.0000000000000000 & 0.0000000000000000\end{array}$

$\begin{array}{lllll}0.0999899999999982 & 0.4999800000000008 & 0.0000000000000000\end{array}$

$\begin{array}{lllll}0.0999899999999982 & 0.1666600000000003 & 0.0000000000000000\end{array}$

$\begin{array}{llll}0.1999700000000004 & 0.3333200000000005 & 0.0000000000000000\end{array}$

$\begin{array}{llll}0.0000000000000000 & 0.3333200000000005 & 0.0000000000000000\end{array}$

$\begin{array}{llll}0.2999599999999987 & 0.4999800000000008 & 0.0000000000000000\end{array}$

$\begin{array}{lllll}0.8998800000000031 & 0.7222200000000001 & 0.0659399999999977\end{array}$

$\begin{array}{lllll}0.4999299999999991 & 0.7222200000000001 & 0.0659399999999977\end{array}$

$\begin{array}{lllll}0.6998999999999995 & 0.7222200000000001 & 0.0659399999999977\end{array}$

$\begin{array}{llll}0.7998899999999978 & 0.8888800000000003 & 0.0659399999999977\end{array}$

$\begin{array}{llll}0.5999199999999973 & 0.8888800000000003 & 0.0659399999999977\end{array}$

$\begin{array}{lllll}0.0999899999999982 & 0.7222200000000001 & 0.0659399999999977\end{array}$

$\begin{array}{lllll}0.2999599999999987 & 0.7222200000000001 & 0.0659399999999977\end{array}$

$\begin{array}{llll}0.3999499999999969 & 0.8888800000000003 & 0.0659399999999977\end{array}$

$\begin{array}{llll}0.1999700000000004 & 0.8888800000000003 & 0.0659399999999977\end{array}$

$\begin{array}{lllll}0.0000000000000000 & 0.8888800000000003 & 0.0659399999999977\end{array}$

$\begin{array}{lllll}0.8998800000000031 & 0.0555499999999967 & 0.0659399999999977\end{array}$

$\begin{array}{lllll}0.4999299999999991 & 0.0555499999999967 & 0.0659399999999977\end{array}$

$\begin{array}{llll}0.6998999999999995 & 0.3888800000000003 & 0.0659399999999977\end{array}$

$\begin{array}{llll}0.6998999999999995 & 0.0555499999999967 & 0.0659399999999977\end{array}$

$\begin{array}{lllll}0.7998899999999978 & 0.2222099999999969 & 0.0659399999999977\end{array}$

$\begin{array}{lllll}0.5999199999999973 & 0.2222099999999969 & 0.0659399999999977\end{array}$

$\begin{array}{lllll}0.8998800000000031 & 0.3888800000000003 & 0.0659399999999977\end{array}$

$\begin{array}{llll}0.7998899999999978 & 0.5555400000000006 & 0.0659399999999977\end{array}$

$\begin{array}{lllll}0.0999899999999982 & 0.0555499999999967 & 0.0659399999999977\end{array}$

$\begin{array}{lllll}0.2999599999999987 & 0.3888800000000003 & 0.0659399999999977\end{array}$

$\begin{array}{lllll}0.2999599999999987 & 0.0555499999999967 & 0.0659399999999977\end{array}$

$\begin{array}{llll}0.3999499999999969 & 0.2222099999999969 & 0.0659399999999977\end{array}$

$\begin{array}{lllll}0.1999700000000004 & 0.2222099999999969 & 0.0659399999999977\end{array}$

$\begin{array}{lllll}0.4999299999999991 & 0.3888800000000003 & 0.0659399999999977\end{array}$

$\begin{array}{lllll}0.5999199999999973 & 0.5555400000000006 & 0.0659399999999977\end{array}$

$\begin{array}{llll}0.3999499999999969 & 0.5555400000000006 & 0.0659399999999977\end{array}$

$\begin{array}{lllll}0.0000000000000000 & 0.2222099999999969 & 0.0659399999999977\end{array}$

$\begin{array}{lllll}0.0999899999999982 & 0.3888800000000003 & 0.0659399999999977\end{array}$

$\begin{array}{lllll}0.1999700000000004 & 0.5555400000000006 & 0.0659399999999977\end{array}$

$\begin{array}{lllll}0.0000000000000000 & 0.5555400000000006 & 0.0659399999999977\end{array}$

$\begin{array}{llll}0.9009243512900024 & 0.9440897073888517 & 0.1329751244588218\end{array}$

$\begin{array}{llll}0.8017527651821883 & 0.7807181950398504 & 0.1325495085259946\end{array}$

$\begin{array}{llll}0.5002768995975391 & 0.9440337803710371 & 0.1316920845247413\end{array}$

$\begin{array}{llll}0.5990400432197145 & 0.7782629395242527 & 0.1363212029050077\end{array}$

$\begin{array}{llll}0.4014421203315215 & 0.7780391905263554 & 0.1363090535744992\end{array}$

$\begin{array}{llll}0.7010074751589462 & 0.9440250403976620 & 0.1326700962509108\end{array}$ 
$\begin{array}{llll}0.0992452549376540 & 0.9441203341712057 & 0.1330264768915042\end{array}$ $\begin{array}{llll}0.1981223444964918 & 0.7807690876631151 & 0.1325233811491415\end{array}$ $\begin{array}{llll}0.9997089489791759 & 0.7800241598048310 & 0.1330955172658001\end{array}$ $\begin{array}{llll}0.2994141912089672 & 0.9439874694305291 & 0.1325930008799655\end{array}$ $\begin{array}{llll}0.9013463645794552 & 0.2737275297181747 & 0.1318728304175896\end{array}$ $\begin{array}{llll}0.8014305551046427 & 0.1077867514491170 & 0.1322712143553640\end{array}$ $\begin{array}{lllll}0.4986781102887797 & 0.2796727958722179 & 0.1281732744587820\end{array}$ $\begin{array}{llll}0.5997630936131677 & 0.1095205074198062 & 0.1367296129655628\end{array}$ $\begin{array}{llll}0.4017547935385091 & 0.1091656224591576 & 0.1367652058222505\end{array}$ $\begin{array}{llll}0.7010597495256286 & 0.6153160178310360 & 0.1342726603854631\end{array}$ $\begin{array}{llll}0.7009268775424052 & 0.2735212268347400 & 0.1342499993614513\end{array}$ $\begin{array}{llll}0.8060824695189167 & 0.4470008791983187 & 0.1363784304460046\end{array}$ $\begin{array}{llll}0.6028477319684423 & 0.4427191495131655 & 0.1299809774386934\end{array}$ $\begin{array}{llll}0.9012687360129370 & 0.6152922674855773 & 0.1315629707747679\end{array}$ $\begin{array}{llll}0.0994765152020250 & 0.2742798086027258 & 0.1319853604260274\end{array}$ $\begin{array}{llll}0.1987750862272339 & 0.1077365800812480 & 0.1323262601675365\end{array}$ $\begin{array}{llll}0.0002189540353837 & 0.1085674856696609 & 0.1329372044945485\end{array}$ $\begin{array}{llll}0.2986886259589545 & 0.6156208313047355 & 0.1341975068154345\end{array}$ $\begin{array}{llll}0.2985824866093729 & 0.2729302102859629 & 0.1345437941099555\end{array}$ $\begin{array}{llll}0.3965487070160910 & 0.4428707840027137 & 0.1299130994692394\end{array}$ $\begin{array}{llll}0.1938344233819120 & 0.4495893655272040 & 0.1360718990647218\end{array}$ $\begin{array}{lllll}0.5004348836819067 & 0.6057586637147402 & 0.1286113512543829\end{array}$ $\begin{array}{llll}0.0000743430710445 & 0.4430502223713576 & 0.1314916850628473\end{array}$ $\begin{array}{llll}0.0971829784896258 & 0.6160691900752076 & 0.1318222665722502\end{array}$ $\begin{array}{llll}0.2738178754914828 & 0.2224087659363728 & 0.2067335872913849\end{array}$ $\begin{array}{llll}0.2721304791691603 & 0.6619091862600481 & 0.2056027161087306\end{array}$ $\begin{array}{llll}0.7289647899510737 & 0.6632930862419363 & 0.2056533865745282\end{array}$ $\begin{array}{llll}0.7293352976042657 & 0.2238124424365129 & 0.2063379326824745\end{array}$ $\begin{array}{llll}0.8616143493295202 & 0.5360558739820357 & 0.2059096598855728\end{array}$ $\begin{array}{llll}0.8613935784918658 & 0.3497283347373930 & 0.2079464553766449\end{array}$ $\begin{array}{llll}0.5925289495604824 & 0.0938672337575637 & 0.2111666472869759\end{array}$ $\begin{array}{llll}0.4107871847182593 & 0.0937181367025145 & 0.2109813070591620\end{array}$ $\begin{array}{llll}0.1418652488406229 & 0.3473550850284318 & 0.2089576058529018\end{array}$ $\begin{array}{llll}0.1405019441327316 & 0.5336443311710781 & 0.2055618593106613\end{array}$ $\begin{array}{llll}0.4089445148287141 & 0.7925874194548502 & 0.2108692176618413\end{array}$ $\begin{array}{llll}0.5914845348189676 & 0.7932842648515422 & 0.2109698364844592\end{array}$ $\begin{array}{llll}0.7039084106535395 & 0.3674692993242294 & 0.2073867419380637\end{array}$ $\begin{array}{llll}0.8007260164802619 & 0.3945685094366378 & 0.2050500612233470\end{array}$ $\begin{array}{llll}0.8014248575750685 & 0.4908141940473648 & 0.2022399911437154\end{array}$ $\begin{array}{lllll}0.7036214999725448 & 0.5192534315738357 & 0.2049115608703191\end{array}$ $\begin{array}{llll}0.6754250910079620 & 0.2753825581345675 & 0.2005140943961843\end{array}$ $\begin{array}{llll}0.5805081450683495 & 0.2424521517844747 & 0.2004808750710021\end{array}$ $\begin{array}{llll}0.4223430931626772 & 0.2419964442975989 & 0.2000455261850817\end{array}$ $\begin{array}{llll}0.4486360882829181 & 0.1453416302838929 & 0.1943269017243269\end{array}$ $\begin{array}{llll}0.5549386214251968 & 0.1455095611967759 & 0.1944365286514118\end{array}$ $\begin{array}{lllll}0.3270908933867979 & 0.2742381568116453 & 0.2004486685467376\end{array}$ $\begin{array}{lllll}0.2985362021983301 & 0.3662853280229367 & 0.2078923942426982\end{array}$ 
$\begin{array}{llll}0.2986384219684441 & 0.5179727969021991 & 0.2036678681524694\end{array}$

$\begin{array}{llll}0.2004964296739356 & 0.4885024137613634 & 0.2011903371997853\end{array}$

$\begin{array}{llll}0.2022312903964080 & 0.3926270505795756 & 0.2063411226086825\end{array}$

$\begin{array}{llll}0.3258279900991236 & 0.6114384096230847 & 0.1979578126215799\end{array}$

$\begin{array}{llll}0.4214888051063712 & 0.6445359965534001 & 0.1989594417251034\end{array}$

$\begin{array}{llll}0.5800437687796250 & 0.6449969919172832 & 0.1993130902941187\end{array}$

$\begin{array}{lllll}0.6755286193735055 & 0.6121366211198711 & 0.1985353753091362\end{array}$

$\begin{array}{llll}0.5536051982164899 & 0.7417783103439726 & 0.1942725589579865\end{array}$

$\begin{array}{llll}0.4473823765910096 & 0.7415168144000509 & 0.1941279710987730\end{array}$

$\begin{array}{llll}0.6449995934411686 & 0.4440297183551026 & 0.2071711990026833\end{array}$

$\begin{array}{llll}0.5014161225004706 & 0.2982129591006851 & 0.2024717469166693\end{array}$

$\begin{array}{lllll}0.5008201940336502 & 0.5885395174007466 & 0.2009807919858127\end{array}$

$\begin{array}{llll}0.3575213610735118 & 0.4432766436260137 & 0.2064616809313514\end{array}$

$\begin{array}{llll}0.5013363438915777 & 0.4431643716087027 & 0.2012610021237876\end{array}$

FeP-Pt(111) - T-0, vdW-DF-optB88:

Pt H C N Fe

1.00000000000000

$\begin{array}{lll}14.0790000000000006 & 0.0000000000000000 & 0.0000000000000000\end{array}$

$\begin{array}{lll}0.0000000000000000 & 14.6310000000000002 & 0.0000000000000000\end{array}$

$\begin{array}{llll}0.0000000000000000 & 0.0000000000000000 & 35.0000000000000000\end{array}$

$\mathrm{Pt} \mathrm{H} \quad \mathrm{C} \quad \mathrm{N}$ Fe

$\begin{array}{lllll}90 & 12 & 20 & 4 & 1\end{array}$

Direct

$0.0000000000000000 \quad 0.0000000000000000 \quad 0.0000000000000000$

$\begin{array}{llll}0.2000000000000028 & 0.0000000000000000 & 0.0000000000000000\end{array}$

$\begin{array}{lllll}0.3999999999999986 & 0.0000000000000000 & 0.0000000000000000\end{array}$

$\begin{array}{llll}0.3000100000000003 & 0.1666799999999995 & 0.0000000000000000\end{array}$

$\begin{array}{llll}0.0000000000000000 & 0.3333399999999997 & 0.0000000000000000\end{array}$

$\begin{array}{llll}0.2000000000000028 & 0.3333399999999997 & 0.0000000000000000\end{array}$

$\begin{array}{llll}0.1000099999999975 & 0.5000199999999992 & 0.0000000000000000\end{array}$

$\begin{array}{lllll}0.1000099999999975 & 0.1666799999999995 & 0.0000000000000000\end{array}$

$\begin{array}{llll}0.0000000000000000 & 0.6666999999999987 & 0.0000000000000000\end{array}$

$\begin{array}{llll}0.6000099999999975 & 0.0000000000000000 & 0.0000000000000000\end{array}$

$\begin{array}{llll}0.8000200000000035 & 0.0000000000000000 & 0.0000000000000000\end{array}$

$\begin{array}{lllll}0.7000099999999989 & 0.1666799999999995 & 0.0000000000000000\end{array}$

$\begin{array}{lllll}0.3999999999999986 & 0.3333399999999997 & 0.0000000000000000\end{array}$

$\begin{array}{llll}0.6000099999999975 & 0.3333399999999997 & 0.0000000000000000\end{array}$

$\begin{array}{llll}0.5000100000000032 & 0.5000199999999992 & 0.0000000000000000\end{array}$

$\begin{array}{llll}0.5000100000000032 & 0.1666799999999995 & 0.0000000000000000\end{array}$

$\begin{array}{lllll}0.2000000000000028 & 0.6666999999999987 & 0.0000000000000000\end{array}$

$\begin{array}{llll}0.3999999999999986 & 0.6666999999999987 & 0.0000000000000000\end{array}$

$\begin{array}{llll}0.3000100000000003 & 0.8333599999999990 & 0.0000000000000000\end{array}$

$\begin{array}{llll}0.3000100000000003 & 0.5000199999999992 & 0.0000000000000000\end{array}$

$\begin{array}{lllll}0.1000099999999975 & 0.8333599999999990 & 0.0000000000000000\end{array}$

$\begin{array}{lllll}0.8000200000000035 & 0.3333399999999997 & 0.0000000000000000\end{array}$

$\begin{array}{llll}0.9000199999999978 & 0.5000199999999992 & 0.0000000000000000\end{array}$ 
$0.9000199999999978 \quad 0.16667999999999950 .0000000000000000$ $\begin{array}{llll}0.6000099999999975 & 0.6666999999999987 & 0.0000000000000000\end{array}$ $\begin{array}{llll}0.8000200000000035 & 0.6666999999999987 & 0.0000000000000000\end{array}$ $\begin{array}{llll}0.7000099999999989 & 0.8333599999999990 & 0.0000000000000000\end{array}$ $\begin{array}{llll}0.7000099999999989 & 0.5000199999999992 & 0.0000000000000000\end{array}$ $\begin{array}{llll}0.5000100000000032 & 0.8333599999999990 & 0.0000000000000000\end{array}$ $\begin{array}{lllll}0.9000199999999978 & 0.8333599999999990 & 0.0000000000000000\end{array}$ $\begin{array}{llll}0.3000100000000003 & 0.9444400000000002 & 0.0656899999999965\end{array}$ $\begin{array}{llll}0.0000000000000000 & 0.1111199999999997 & 0.0656899999999965\end{array}$ $\begin{array}{lllll}0.2000000000000028 & 0.1111199999999997 & 0.0656899999999965\end{array}$ $\begin{array}{lllll}0.1000099999999975 & 0.2777900000000031 & 0.0656899999999965\end{array}$ $\begin{array}{llll}0.1000099999999975 & 0.9444400000000002 & 0.0656899999999965\end{array}$ $\begin{array}{llll}0.0000000000000000 & 0.4444599999999994 & 0.0656899999999965\end{array}$ $\begin{array}{llll}0.7000099999999989 & 0.9444400000000002 & 0.0656899999999965\end{array}$ $\begin{array}{llll}0.3999999999999986 & 0.1111199999999997 & 0.0656899999999965\end{array}$ $\begin{array}{llll}0.6000200000000007 & 0.1111199999999997 & 0.0656899999999965\end{array}$ $\begin{array}{lllll}0.5000100000000032 & 0.2777900000000031 & 0.0656899999999965\end{array}$ $\begin{array}{llll}0.5000100000000032 & 0.9444400000000002 & 0.0656899999999965\end{array}$ $\begin{array}{lllll}0.2000000000000028 & 0.4444599999999994 & 0.0656899999999965\end{array}$ $\begin{array}{llll}0.3999999999999986 & 0.4444599999999994 & 0.0656899999999965\end{array}$ $\begin{array}{llll}0.3000100000000003 & 0.6111399999999989 & 0.0656899999999965\end{array}$ $\begin{array}{llll}0.3000100000000003 & 0.2777900000000031 & 0.0656899999999965\end{array}$ $\begin{array}{llll}0.0000000000000000 & 0.7778100000000023 & 0.0656899999999965\end{array}$ $\begin{array}{llll}0.2000000000000028 & 0.7778100000000023 & 0.0656899999999965\end{array}$ $\begin{array}{llll}0.1000099999999975 & 0.6111399999999989 & 0.0656899999999965\end{array}$ $\begin{array}{lllll}0.8000200000000035 & 0.1111199999999997 & 0.0656899999999965\end{array}$ $\begin{array}{llll}0.9000199999999978 & 0.2777900000000031 & 0.0656899999999965\end{array}$ $\begin{array}{llll}0.9000199999999978 & 0.9444400000000002 & 0.0656899999999965\end{array}$ $\begin{array}{llll}0.6000200000000007 & 0.4444599999999994 & 0.0656899999999965\end{array}$ $\begin{array}{llll}0.8000200000000035 & 0.4444599999999994 & 0.0656899999999965\end{array}$ $\begin{array}{llll}0.7000099999999989 & 0.6111399999999989 & 0.0656899999999965\end{array}$ $\begin{array}{lllll}0.7000099999999989 & 0.2777900000000031 & 0.0656899999999965\end{array}$ $\begin{array}{llll}0.3999999999999986 & 0.7778100000000023 & 0.0656899999999965\end{array}$ $\begin{array}{llll}0.6000200000000007 & 0.7778100000000023 & 0.0656899999999965\end{array}$ $\begin{array}{llll}0.5000100000000032 & 0.6111399999999989 & 0.0656899999999965\end{array}$ $\begin{array}{llll}0.8000200000000035 & 0.7778100000000023 & 0.0656899999999965\end{array}$ $\begin{array}{llll}0.9000199999999978 & 0.6111399999999989 & 0.0656899999999965\end{array}$ $\begin{array}{llll}0.0003441077381529 & 0.8933884366828132 & 0.1319341325042621\end{array}$ $\begin{array}{lllll}0.1999700951616276 & 0.8874843035859461 & 0.1390599383157678\end{array}$ $\begin{array}{llll}0.1024464465077579 & 0.0577525378564247 & 0.1319708300282607\end{array}$ $\begin{array}{llll}0.0003617859762168 & 0.2221558648923648 & 0.1316343721729435\end{array}$ $\begin{array}{llll}0.4010515421305595 & 0.8899973525081208 & 0.1376842552992977\end{array}$ $\begin{array}{llll}0.6023583138024464 & 0.8873027671987640 & 0.1386731733115718\end{array}$ $\begin{array}{llll}0.5006678981638136 & 0.0565659778670744 & 0.1317334069604535\end{array}$ $\begin{array}{llll}0.1995763039660626 & 0.2283504429789626 & 0.1399129082227304\end{array}$ $\begin{array}{llll}0.4008238843627084 & 0.2235620151348717 & 0.1383492780454524\end{array}$ $\begin{array}{lllll}0.3031690401263805 & 0.3870320975093867 & 0.1285244707777700\end{array}$ 
$\begin{array}{llll}0.3001791331258367 & 0.0566468331553643 & 0.1317594498571328\end{array}$ $\begin{array}{llll}0.9955117193503819 & 0.5564153395758112 & 0.1306922385204404\end{array}$ $\begin{array}{llll}0.1901267133817306 & 0.5572994435191074 & 0.1330289031468768\end{array}$ $\begin{array}{llll}0.0972159285813437 & 0.7261252616292543 & 0.1354337895108729\end{array}$ $\begin{array}{llll}0.0972868688019212 & 0.3893371905432310 & 0.1351223541689894\end{array}$ $\begin{array}{llll}0.8012774367237764 & 0.8935535191903929 & 0.1318508879596713\end{array}$ $\begin{array}{llll}0.9007155806716619 & 0.0576304062854650 & 0.1324024606338567\end{array}$ $\begin{array}{llll}0.6015920920420806 & 0.2279766795721983 & 0.1395578401793732\end{array}$ $\begin{array}{llll}0.8005843254533193 & 0.2217960644931787 & 0.1316375453490727\end{array}$ $\begin{array}{llll}0.7039928777559006 & 0.3889099795379747 & 0.1349185762017316\end{array}$ $\begin{array}{lllll}0.6984094438213411 & 0.0574368791210084 & 0.1319481270614329\end{array}$ $\begin{array}{llll}0.4005307887402267 & 0.5574148360440374 & 0.1294233564332734\end{array}$ $\begin{array}{llll}0.6105357867685874 & 0.5571251491237085 & 0.1330634317230647\end{array}$ $\begin{array}{llll}0.4999064010170638 & 0.7263491414847207 & 0.1286774268647165\end{array}$ $\begin{array}{llll}0.4981725467824167 & 0.3871526081512763 & 0.1285373765629297\end{array}$ $\begin{array}{lllll}0.3013892231010793 & 0.7265380960496096 & 0.1288049979782787\end{array}$ $\begin{array}{llll}0.8051694246623099 & 0.5561638445120582 & 0.1306879931738907\end{array}$ $\begin{array}{llll}0.9007732567635161 & 0.7267612542427139 & 0.1320022839738814\end{array}$ $\begin{array}{llll}0.9005955957984485 & 0.3881384272180067 & 0.1322427509406864\end{array}$ $\begin{array}{llll}0.7044839461564463 & 0.7255127370446021 & 0.1348002424387163\end{array}$ $\begin{array}{lllll}0.7182946880589686 & 0.5581397939326804 & 0.2000054867083109\end{array}$ $\begin{array}{llll}0.4005176025388276 & 0.2494856568184398 & 0.2122675882080945\end{array}$ $\begin{array}{llll}0.0845438376690735 & 0.5595675605781242 & 0.2002364396624452\end{array}$ $\begin{array}{llll}0.4020901595808348 & 0.8678109628866377 & 0.2120642751826393\end{array}$ $\begin{array}{llll}0.0821815651630541 & 0.7427132618702188 & 0.2105925834901636\end{array}$ $\begin{array}{llll}0.2127193358205893 & 0.8652038296029885 & 0.2127528101906673\end{array}$ $\begin{array}{llll}0.5914386091470900 & 0.8643773472849234 & 0.2124377386824501\end{array}$ $\begin{array}{llll}0.7214942578237982 & 0.7414845504216225 & 0.2098601838556562\end{array}$ $\begin{array}{llll}0.7203418910378715 & 0.3762686236120815 & 0.2101191029026287\end{array}$ $\begin{array}{llll}0.5904373898511694 & 0.2533401838946646 & 0.2130953357586432\end{array}$ $\begin{array}{lllll}0.2112022110064238 & 0.2541934039302931 & 0.2132981205367666\end{array}$ $\begin{array}{llll}0.0818027710721853 & 0.3775924892641487 & 0.2103165810479929\end{array}$ $\begin{array}{llll}0.3108939977609213 & 0.7589517883437203 & 0.1892348520845011\end{array}$ $\begin{array}{llll}0.2178807319530081 & 0.8096928058295254 & 0.1915670127558232\end{array}$ $\begin{array}{llll}0.1465775487770558 & 0.7334258853213126 & 0.1933623098255005\end{array}$ $\begin{array}{llll}0.2040947447773669 & 0.6489610267986140 & 0.2011181344330918\end{array}$ $\begin{array}{llll}0.4019748441124519 & 0.8106722214347968 & 0.1914571168888605\end{array}$ $\begin{array}{llll}0.4927667512939876 & 0.7584919135403607 & 0.1891632158299986\end{array}$ $\begin{array}{llll}0.5991113976599726 & 0.6482114340852283 & 0.2007887277811954\end{array}$ $\begin{array}{llll}0.6567249368774530 & 0.7323763845175175 & 0.1928501286678975\end{array}$ $\begin{array}{llll}0.5857864730396761 & 0.8089579259389718 & 0.1912229596407354\end{array}$ $\begin{array}{lllll}0.6406261834947771 & 0.5585458495800897 & 0.1979869210923119\end{array}$ $\begin{array}{llll}0.5981916929183411 & 0.4691302752296920 & 0.2005077047235535\end{array}$ $\begin{array}{lllll}0.4916453461108555 & 0.3584385352797177 & 0.1891740002013786\end{array}$ $\begin{array}{llll}0.5848570527184035 & 0.3080494871635673 & 0.1915452812653552\end{array}$ $\begin{array}{llll}0.6558448980676402 & 0.3846445176094601 & 0.1928872664874613\end{array}$ $\begin{array}{llll}0.4007005843418281 & 0.3064952197920511 & 0.1915798851854102\end{array}$ 
$\begin{array}{llll}0.3101459566668144 & 0.3589429603455401 & 0.1891279483849753\end{array}$

$\begin{array}{llll}0.2042688597403394 & 0.4701106199012032 & 0.2005018627202787\end{array}$

$\begin{array}{llll}0.1621896181026393 & 0.5596639658482812 & 0.1980639160958711\end{array}$

$\begin{array}{llll}0.1461393845549826 & 0.3857582253532482 & 0.1929693288002912\end{array}$

$\begin{array}{llll}0.2168481250252131 & 0.3088453117397710 & 0.1917236031936821\end{array}$

$\begin{array}{llll}0.2979073994342076 & 0.6663567218555084 & 0.2032264180060649\end{array}$

$\begin{array}{llll}0.5053884333475065 & 0.6659921456096890 & 0.2031743619794982\end{array}$

$\begin{array}{llll}0.2978781331535284 & 0.4518129040469984 & 0.2030354802180819\end{array}$

$\begin{array}{llll}0.5044525098203664 & 0.4512933247400240 & 0.2030759464836609\end{array}$

$\begin{array}{llll}0.4013854143083558 & 0.5588449579002385 & 0.2000533746486757\end{array}$

\section{FeP-Pt(111) - T-45, vdW-DF-optB88:}

$\mathrm{Pt} \mathrm{H} \mathrm{C} \mathrm{N} \mathrm{Fe}$

1.00000000000000

$\begin{array}{lll}14.0790000000000006 & 0.0000000000000000 & 0.0000000000000000\end{array}$

$\begin{array}{llll}0.0000000000000000 & 14.6310000000000002 & 0.0000000000000000\end{array}$

$\begin{array}{llll}0.0000000000000000 & 0.0000000000000000 & 35.0000000000000000\end{array}$

Pt $\mathrm{H} \quad \mathrm{C} \quad \mathrm{N} \quad \mathrm{Fe}$

$\begin{array}{lllll}90 & 12 & 20 & 4 & 1\end{array}$

Direct

$\begin{array}{llll}0.0000000000000000 & 0.0000000000000000 & 0.0000000000000000\end{array}$

$\begin{array}{lllll}0.2000000000000028 & 0.0000000000000000 & 0.0000000000000000\end{array}$

$\begin{array}{llllll}0.3999999999999986 & 0.0000000000000000 & 0.0000000000000000\end{array}$

$\begin{array}{lllll}0.3000100000000003 & 0.1666799999999995 & 0.0000000000000000\end{array}$

$\begin{array}{lllll}0.0000000000000000 & 0.3333399999999999 & 0.0000000000000000\end{array}$

$\begin{array}{llll}0.2000000000000028 & 0.33333399999999997 & 0.0000000000000000\end{array}$

$\begin{array}{lllll}0.1000099999999975 & 0.5000199999999992 & 0.0000000000000000\end{array}$

$\begin{array}{lllll}0.1000099999999975 & 0.1666799999999995 & 0.0000000000000000\end{array}$

$\begin{array}{lllll}0.0000000000000000 & 0.6666999999999987 & 0.0000000000000000\end{array}$

$\begin{array}{lllll}0.6000099999999975 & 0.0000000000000000 & 0.0000000000000000\end{array}$

$\begin{array}{lllll}0.8000200000000035 & 0.0000000000000000 & 0.0000000000000000\end{array}$

$\begin{array}{lllll}0.7000099999999989 & 0.1666799999999995 & 0.0000000000000000\end{array}$

$\begin{array}{lllll}0.3999999999999986 & 0.3333399999999997 & 0.0000000000000000\end{array}$

$\begin{array}{lllll}0.6000099999999975 & 0.33333399999999997 & 0.0000000000000000\end{array}$

$\begin{array}{lllll}0.5000100000000032 & 0.5000199999999992 & 0.0000000000000000\end{array}$

$\begin{array}{llll}0.5000100000000032 & 0.1666799999999995 & 0.0000000000000000\end{array}$

$\begin{array}{lllll}0.2000000000000028 & 0.6666999999999987 & 0.0000000000000000\end{array}$

$\begin{array}{lllll}0.3999999999999986 & 0.6666999999999987 & 0.0000000000000000\end{array}$

$\begin{array}{lllll}0.3000100000000003 & 0.8333599999999990 & 0.0000000000000000\end{array}$

$\begin{array}{llll}0.3000100000000003 & 0.5000199999999992 & 0.0000000000000000\end{array}$

$\begin{array}{lllll}0.1000099999999975 & 0.8333599999999990 & 0.0000000000000000\end{array}$

$\begin{array}{lllll}0.8000200000000035 & 0.3333399999999997 & 0.0000000000000000\end{array}$

$\begin{array}{lllll}0.9000199999999978 & 0.5000199999999992 & 0.0000000000000000\end{array}$

$\begin{array}{llll}0.9000199999999978 & 0.1666799999999995 & 0.0000000000000000\end{array}$

$\begin{array}{llll}0.6000099999999975 & 0.6666999999999987 & 0.0000000000000000\end{array}$

$\begin{array}{lllll}0.8000200000000035 & 0.6666999999999987 & 0.0000000000000000\end{array}$

$\begin{array}{lllll}0.7000099999999989 & 0.8333599999999990 & 0.0000000000000000\end{array}$ 
$\begin{array}{llll}0.7000099999999989 & 0.5000199999999992 & 0.0000000000000000\end{array}$

$\begin{array}{lllll}0.5000100000000032 & 0.8333599999999990 & 0.0000000000000000\end{array}$

$\begin{array}{lllll}0.9000199999999978 & 0.8333599999999990 & 0.0000000000000000\end{array}$

$\begin{array}{llll}0.3000100000000003 & 0.9444400000000002 & 0.0656899999999965\end{array}$

$\begin{array}{lllll}0.0000000000000000 & 0.1111199999999997 & 0.0656899999999965\end{array}$

$\begin{array}{llll}0.2000000000000028 & 0.1111199999999997 & 0.0656899999999965\end{array}$

$\begin{array}{lllll}0.1000099999999975 & 0.2777900000000031 & 0.0656899999999965\end{array}$

$\begin{array}{llll}0.1000099999999975 & 0.9444400000000002 & 0.0656899999999965\end{array}$

$\begin{array}{llll}0.0000000000000000 & 0.4444599999999994 & 0.0656899999999965\end{array}$

$\begin{array}{llll}0.7000099999999989 & 0.9444400000000002 & 0.0656899999999965\end{array}$

$\begin{array}{llll}0.3999999999999986 & 0.1111199999999997 & 0.0656899999999965\end{array}$

$\begin{array}{llll}0.6000200000000007 & 0.1111199999999997 & 0.0656899999999965\end{array}$

$\begin{array}{llll}0.5000100000000032 & 0.2777900000000031 & 0.0656899999999965\end{array}$

$\begin{array}{llll}0.5000100000000032 & 0.9444400000000002 & 0.0656899999999965\end{array}$

$\begin{array}{llll}0.2000000000000028 & 0.4444599999999994 & 0.0656899999999965\end{array}$

$\begin{array}{llll}0.3999999999999986 & 0.4444599999999994 & 0.0656899999999965\end{array}$

$\begin{array}{lllll}0.3000100000000003 & 0.6111399999999989 & 0.0656899999999965\end{array}$

$\begin{array}{lllll}0.3000100000000003 & 0.2777900000000031 & 0.0656899999999965\end{array}$

$\begin{array}{llll}0.0000000000000000 & 0.7778100000000023 & 0.0656899999999965\end{array}$

$\begin{array}{llll}0.2000000000000028 & 0.7778100000000023 & 0.0656899999999965\end{array}$

$\begin{array}{llll}0.1000099999999975 & 0.6111399999999989 & 0.0656899999999965\end{array}$

$\begin{array}{lllll}0.8000200000000035 & 0.1111199999999997 & 0.0656899999999965\end{array}$

$\begin{array}{lllll}0.9000199999999978 & 0.2777900000000031 & 0.0656899999999965\end{array}$

$\begin{array}{llll}0.9000199999999978 & 0.9444400000000002 & 0.0656899999999965\end{array}$

$\begin{array}{llll}0.6000200000000007 & 0.4444599999999994 & 0.0656899999999965\end{array}$

$\begin{array}{lllll}0.8000200000000035 & 0.4444599999999994 & 0.0656899999999965\end{array}$

$\begin{array}{llll}0.7000099999999989 & 0.6111399999999989 & 0.0656899999999965\end{array}$

$\begin{array}{llllll}0.7000099999999989 & 0.2777900000000031 & 0.0656899999999965\end{array}$

$\begin{array}{llll}0.3999999999999986 & 0.7778100000000023 & 0.0656899999999965\end{array}$

$\begin{array}{llll}0.6000200000000007 & 0.7778100000000023 & 0.0656899999999965\end{array}$

$\begin{array}{llll}0.5000100000000032 & 0.6111399999999989 & 0.0656899999999965\end{array}$

$\begin{array}{llll}0.8000200000000035 & 0.7778100000000023 & 0.0656899999999965\end{array}$

$\begin{array}{llll}0.9000199999999978 & 0.6111399999999989 & 0.0656899999999965\end{array}$

$\begin{array}{llll}0.9983472978694358 & 0.8927751608727341 & 0.1326163710073491\end{array}$

$\begin{array}{llll}0.1951950871916915 & 0.8939900616371200 & 0.1305358626003563\end{array}$

$\begin{array}{llll}0.0984215713216515 & 0.0585257668427215 & 0.1326729777718947\end{array}$

$\begin{array}{llll}0.9979840740627041 & 0.2244866797913918 & 0.1326670663450463\end{array}$

$\begin{array}{llll}0.4000755815613242 & 0.8907042770062381 & 0.1367942098976628\end{array}$

$\begin{array}{llll}0.6050596017876083 & 0.8941117343986795 & 0.1304955102413277\end{array}$

$\begin{array}{llll}0.5007944174287188 & 0.0597766984872042 & 0.1311362674518179\end{array}$

$\begin{array}{llll}0.1950253552535859 & 0.2231215978808917 & 0.1309571177059810\end{array}$

$\begin{array}{llll}0.4010925675672545 & 0.2311295295416152 & 0.1379454720758407\end{array}$

$\begin{array}{llll}0.2916644082615728 & 0.3921194042456114 & 0.1328442619419211\end{array}$

$\begin{array}{llll}0.2997581823017563 & 0.0599517470287043 & 0.1311721053337323\end{array}$

$\begin{array}{llll}0.9982750543446665 & 0.5583728977275235 & 0.1326755833315853\end{array}$

$\begin{array}{llll}0.1957046252078530 & 0.5585563433805534 & 0.1296627599725473\end{array}$

$\begin{array}{llll}0.0951855202770821 & 0.7255677529068604 & 0.1323980931287717\end{array}$ 
$\begin{array}{llll}0.0947665456053789 & 0.3909791180032196 & 0.1320446730939662\end{array}$ $\begin{array}{llll}0.8020928810335336 & 0.8924215650646912 & 0.1325476620368875\end{array}$ $\begin{array}{llll}0.9001241956248845 & 0.0585161001568223 & 0.1325831377330077\end{array}$ $\begin{array}{llll}0.6059945995064013 & 0.2233953791855561 & 0.1308034834120902\end{array}$ $\begin{array}{llll}0.8030023966646895 & 0.2245209074830332 & 0.1325908550298172\end{array}$ $\begin{array}{llll}0.7060913358391219 & 0.3911980982655733 & 0.1321643253878846\end{array}$ $\begin{array}{llll}0.7020607067482590 & 0.0585654917514287 & 0.1326406852655879\end{array}$ $\begin{array}{llll}0.3998975770766949 & 0.5589778762442333 & 0.1312879611429703\end{array}$ $\begin{array}{llll}0.6048396800666346 & 0.5589021779237047 & 0.1295418099734247\end{array}$ $\begin{array}{llll}0.5065536101850644 & 0.7251900914995847 & 0.1327953310884050\end{array}$ $\begin{array}{lllll}0.5094224127860827 & 0.3927247003313544 & 0.1328083680106584\end{array}$ $\begin{array}{llll}0.2937308735081797 & 0.7252410372649418 & 0.1329848937016371\end{array}$ $\begin{array}{llll}0.8022986454133882 & 0.5582177438645540 & 0.1326766136204132\end{array}$ $\begin{array}{llll}0.9003617298368419 & 0.7253094205418474 & 0.1327816300312085\end{array}$ $\begin{array}{llll}0.9004405352831814 & 0.3920038082599989 & 0.1328794100392676\end{array}$ $\begin{array}{llll}0.7052456963334626 & 0.7256165872500868 & 0.1324077660523034\end{array}$ $\begin{array}{llll}0.6294032272897269 & 0.7802275576746769 & 0.2092941974120727\end{array}$ $\begin{array}{llll}0.6318713512062887 & 0.3455954153054970 & 0.2097821390129424\end{array}$ $\begin{array}{llll}0.1744409832960755 & 0.3446089143429608 & 0.2094337433363680\end{array}$ $\begin{array}{lllll}0.1727459686295610 & 0.7788226962045631 & 0.2093491519757611\end{array}$ $\begin{array}{llll}0.0454616374588321 & 0.4689369346295395 & 0.2286889888420202\end{array}$ $\begin{array}{llll}0.0448154008568478 & 0.6539776303415065 & 0.2289171296506379\end{array}$ $\begin{array}{llll}0.3047118618331370 & 0.9089124658101473 & 0.2050383141169269\end{array}$ $\begin{array}{llll}0.4966412437025587 & 0.9095658488910675 & 0.2047574790183473\end{array}$ $\begin{array}{llll}0.7586101546179091 & 0.6566583927408211 & 0.2291362820694182\end{array}$ $\begin{array}{lllll}0.7602535362596242 & 0.4715327039377575 & 0.2290073209672369\end{array}$ $\begin{array}{llll}0.5000057884599048 & 0.2150653898800758 & 0.2055457913262471\end{array}$ $\begin{array}{llll}0.3078439156116247 & 0.2144843564515903 & 0.2064175359257095\end{array}$ $\begin{array}{llll}0.2016648463384184 & 0.6375720512644873 & 0.2190092799374544\end{array}$ $\begin{array}{lllll}0.1055286152505604 & 0.6084324921166000 & 0.2262611431566021\end{array}$ $\begin{array}{lllll}0.1058865756721962 & 0.5149078449207934 & 0.2262029292385347\end{array}$ $\begin{array}{llll}0.2022495979259598 & 0.4861853523055372 & 0.2188926476752359\end{array}$ $\begin{array}{llll}0.2282198288805169 & 0.7266084222324615 & 0.2091087083811785\end{array}$ $\begin{array}{llll}0.3212540075490604 & 0.7558484045096001 & 0.1989989496651661\end{array}$ $\begin{array}{llll}0.4811737706459072 & 0.7562889054735813 & 0.1989201474397535\end{array}$ $\begin{array}{llll}0.4515176448889331 & 0.8537328480031360 & 0.1949897599029108\end{array}$ $\begin{array}{lllll}0.3500791793962127 & 0.8533788667696393 & 0.1951391345574553\end{array}$ $\begin{array}{llll}0.5744962634181793 & 0.7274336821038965 & 0.2089293352071179\end{array}$ $\begin{array}{llll}0.6021084070576308 & 0.6385596254401031 & 0.2188154330563391\end{array}$ $\begin{array}{llll}0.6032535393712469 & 0.4871252044997980 & 0.2187444359816724\end{array}$ $\begin{array}{lllll}0.6993211155179625 & 0.5167950826639042 & 0.2262008377813899\end{array}$ $\begin{array}{lllll}0.6985300530176896 & 0.6103540566148904 & 0.2263454782911779\end{array}$ $\begin{array}{llll}0.5763998594071598 & 0.3978249895591952 & 0.2093095081549352\end{array}$ $\begin{array}{llll}0.4830794282784606 & 0.3680986643299812 & 0.1993654674010156\end{array}$ $\begin{array}{llll}0.3232212649596758 & 0.3675200922769746 & 0.1999584478813590\end{array}$ $\begin{array}{llll}0.2297564127649965 & 0.3969824446259088 & 0.2093113347199891\end{array}$ $\begin{array}{llll}0.3527620971272383 & 0.2700257929751206 & 0.1962196096186225\end{array}$ 
$\begin{array}{llll}0.4542090018394687 & 0.2705006516345455 & 0.1959019962457288\end{array}$

$\begin{array}{llll}0.2603379236078261 & 0.5620607514801597 & 0.2166006305366351\end{array}$

$\begin{array}{llll}0.4014035063777942 & 0.7026086925568328 & 0.2052582662138707\end{array}$

$\begin{array}{llll}0.4028595208431069 & 0.4213402351799702 & 0.2057087393080606\end{array}$

$\begin{array}{llll}0.5444079487485179 & 0.5625411097484895 & 0.2162078187390355\end{array}$

$\begin{array}{llll}0.4022196525487426 & 0.5618638155917843 & 0.2027877775593899\end{array}$

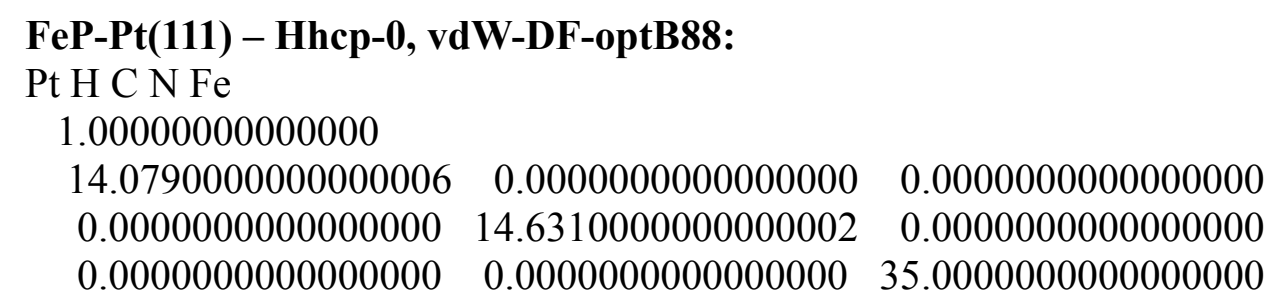

$\begin{array}{lllll}\mathrm{Pt} & \mathrm{H} & \mathrm{C} & \mathrm{N} & \mathrm{Fe}\end{array}$

$\begin{array}{lllll}90 & 12 & 20 & 4 & 1\end{array}$

Direct

$0.0000000000000000 \quad 0.0000000000000000 \quad 0.00000000000000000$

$\begin{array}{lllll}0.2000000000000028 & 0.0000000000000000 & 0.0000000000000000\end{array}$

$\begin{array}{lllll}0.3999999999999986 & 0.0000000000000000 & 0.0000000000000000\end{array}$

$\begin{array}{lllll}0.3000100000000003 & 0.1666799999999995 & 0.0000000000000000\end{array}$

$\begin{array}{lllll}0.0000000000000000 & 0.3333399999999997 & 0.0000000000000000\end{array}$

$\begin{array}{lllll}0.2000000000000028 & 0.3333399999999997 & 0.0000000000000000\end{array}$

$\begin{array}{lllll}0.1000099999999975 & 0.5000199999999992 & 0.0000000000000000\end{array}$

$\begin{array}{lllll}0.1000099999999975 & 0.1666799999999995 & 0.0000000000000000\end{array}$

$\begin{array}{lllll}0.0000000000000000 & 0.6666999999999987 & 0.0000000000000000\end{array}$

$\begin{array}{lllll}0.6000099999999975 & 0.0000000000000000 & 0.0000000000000000\end{array}$

$\begin{array}{lllll}0.8000200000000035 & 0.0000000000000000 & 0.0000000000000000\end{array}$

$\begin{array}{lllll}0.7000099999999989 & 0.1666799999999995 & 0.0000000000000000\end{array}$

$\begin{array}{lllll}0.3999999999999986 & 0.33333399999999997 & 0.0000000000000000\end{array}$

$\begin{array}{lllll}0.6000099999999975 & 0.33333999999999997 & 0.0000000000000000\end{array}$

$\begin{array}{llll}0.5000100000000032 & 0.5000199999999992 & 0.0000000000000000\end{array}$

$\begin{array}{llll}0.5000100000000032 & 0.1666799999999995 & 0.0000000000000000\end{array}$

$\begin{array}{lllll}0.2000000000000028 & 0.6666999999999987 & 0.0000000000000000\end{array}$

$\begin{array}{lllll}0.3999999999999986 & 0.6666999999999987 & 0.0000000000000000\end{array}$

$\begin{array}{lllll}0.3000100000000003 & 0.8333599999999990 & 0.0000000000000000\end{array}$

$\begin{array}{lllll}0.3000100000000003 & 0.5000199999999992 & 0.0000000000000000\end{array}$

$\begin{array}{lllll}0.1000099999999975 & 0.8333599999999990 & 0.0000000000000000\end{array}$

$\begin{array}{llll}0.8000200000000035 & 0.3333399999999997 & 0.0000000000000000\end{array}$

$\begin{array}{lllll}0.9000199999999978 & 0.5000199999999992 & 0.0000000000000000\end{array}$

$\begin{array}{lllll}0.9000199999999978 & 0.1666799999999995 & 0.0000000000000000\end{array}$

$\begin{array}{llll}0.6000099999999975 & 0.6666999999999987 & 0.0000000000000000\end{array}$

$\begin{array}{lllll}0.8000200000000035 & 0.6666999999999987 & 0.0000000000000000\end{array}$

$\begin{array}{lllll}0.7000099999999989 & 0.8333599999999990 & 0.0000000000000000\end{array}$

$\begin{array}{llll}0.7000099999999989 & 0.5000199999999992 & 0.0000000000000000\end{array}$

$\begin{array}{lllll}0.5000100000000032 & 0.8333599999999990 & 0.0000000000000000\end{array}$

$\begin{array}{lllll}0.9000199999999978 & 0.83335999999999990 & 0.0000000000000000\end{array}$

$\begin{array}{lllll}0.3000100000000003 & 0.9444400000000002 & 0.0656899999999965\end{array}$ 
$\begin{array}{llll}0.0000000000000000 & 0.1111199999999997 & 0.0656899999999965\end{array}$

$\begin{array}{lllll}0.2000000000000028 & 0.1111199999999997 & 0.0656899999999965\end{array}$

$\begin{array}{lllll}0.1000099999999975 & 0.2777900000000031 & 0.0656899999999965\end{array}$

$\begin{array}{llll}0.1000099999999975 & 0.9444400000000002 & 0.0656899999999965\end{array}$

$\begin{array}{lllll}0.0000000000000000 & 0.4444599999999994 & 0.0656899999999965\end{array}$

$\begin{array}{llll}0.7000099999999989 & 0.9444400000000002 & 0.0656899999999965\end{array}$

$\begin{array}{lllll}0.3999999999999986 & 0.1111199999999997 & 0.0656899999999965\end{array}$

$\begin{array}{lllll}0.6000200000000007 & 0.1111199999999997 & 0.0656899999999965\end{array}$

$\begin{array}{lllll}0.5000100000000032 & 0.2777900000000031 & 0.0656899999999965\end{array}$

$\begin{array}{llll}0.5000100000000032 & 0.9444400000000002 & 0.0656899999999965\end{array}$

$\begin{array}{llll}0.2000000000000028 & 0.4444599999999994 & 0.0656899999999965\end{array}$

$\begin{array}{llll}0.3999999999999986 & 0.4444599999999994 & 0.0656899999999965\end{array}$

$\begin{array}{llll}0.3000100000000003 & 0.6111399999999989 & 0.0656899999999965\end{array}$

$\begin{array}{lllll}0.3000100000000003 & 0.2777900000000031 & 0.0656899999999965\end{array}$

$\begin{array}{llll}0.0000000000000000 & 0.7778100000000023 & 0.0656899999999965\end{array}$

$\begin{array}{llll}0.2000000000000028 & 0.7778100000000023 & 0.0656899999999965\end{array}$

$\begin{array}{llll}0.1000099999999975 & 0.6111399999999989 & 0.0656899999999965\end{array}$

$\begin{array}{llll}0.8000200000000035 & 0.1111199999999997 & 0.0656899999999965\end{array}$

$\begin{array}{lllll}0.9000199999999978 & 0.2777900000000031 & 0.0656899999999965\end{array}$

$\begin{array}{llll}0.9000199999999978 & 0.9444400000000002 & 0.0656899999999965\end{array}$

$\begin{array}{llll}0.6000200000000007 & 0.4444599999999994 & 0.0656899999999965\end{array}$

$\begin{array}{lll}0.8000200000000035 & 0.4444599999999994 & 0.0656899999999965\end{array}$

$\begin{array}{llll}0.7000099999999989 & 0.6111399999999989 & 0.0656899999999965\end{array}$

$\begin{array}{lllll}0.7000099999999989 & 0.2777900000000031 & 0.0656899999999965\end{array}$

$\begin{array}{llll}0.3999999999999986 & 0.7778100000000023 & 0.0656899999999965\end{array}$

$\begin{array}{llll}0.6000200000000007 & 0.7778100000000023 & 0.0656899999999965\end{array}$

$\begin{array}{llll}0.5000100000000032 & 0.6111399999999989 & 0.0656899999999965\end{array}$

$\begin{array}{llll}0.8000200000000035 & 0.7778100000000023 & 0.0656899999999965\end{array}$

$\begin{array}{llll}0.9000199999999978 & 0.6111399999999989 & 0.0656899999999965\end{array}$

$\begin{array}{llll}0.0000220064323065 & 0.8914052862886942 & 0.1327008340355749\end{array}$

$\begin{array}{llll}0.2004326272111189 & 0.8905366645467894 & 0.1326198175486854\end{array}$

$\begin{array}{llll}0.0980207721275137 & 0.0541453113320212 & 0.1323897123284784\end{array}$

$\begin{array}{llll}0.0007455408102736 & 0.2256949868936606 & 0.1312684431447597\end{array}$

$\begin{array}{llll}0.4026960363597806 & 0.8896216136736713 & 0.1328013936596665\end{array}$

$\begin{array}{llll}0.6031105224719098 & 0.8896410543004408 & 0.1326545513727780\end{array}$

$\begin{array}{llll}0.5035809197821798 & 0.0524518886727619 & 0.1312539781944838\end{array}$

$\begin{array}{llll}0.1990721902450792 & 0.2146938968096782 & 0.1339432144102234\end{array}$

$\begin{array}{lllll}0.4029480204416261 & 0.2182980048793427 & 0.1317893805366872\end{array}$

$\begin{array}{llll}0.3056597940466197 & 0.3908756097149047 & 0.1311616896181093\end{array}$

$\begin{array}{llll}0.3010804662636805 & 0.0509439777006296 & 0.1315341332143722\end{array}$

$\begin{array}{llll}0.9961671174602375 & 0.5610857560974370 & 0.1307713205383481\end{array}$

$\begin{array}{llll}0.2024763649077832 & 0.5639123823552370 & 0.1329890303288560\end{array}$

$\begin{array}{llll}0.0999343989065870 & 0.7276393884266410 & 0.1321536774287670\end{array}$

$\begin{array}{llll}0.1023770214275888 & 0.4027345951858816 & 0.1363250613159245\end{array}$

$\begin{array}{llll}0.8016726596503252 & 0.8911271490010290 & 0.1325967417945029\end{array}$

$\begin{array}{llll}0.9007392915783796 & 0.0568979053859401 & 0.1325220782492436\end{array}$

$\begin{array}{lllll}0.6047790077705641 & 0.2204371630261098 & 0.1354236570199055\end{array}$ 
$\begin{array}{llll}0.8042119822985079 & 0.2234143949370201 & 0.1319362777698381\end{array}$

$\begin{array}{llll}0.7046770513034720 & 0.3906652165382097 & 0.1313774304887186\end{array}$

$\begin{array}{llll}0.7027739039661179 & 0.0552092736108196 & 0.1323418320131954\end{array}$

$\begin{array}{llll}0.4055219542631327 & 0.5607518559608432 & 0.1318037876759681\end{array}$

$\begin{array}{llll}0.6058051440714902 & 0.5607449822058874 & 0.1319095538872011\end{array}$

$\begin{array}{llll}0.5041810041504914 & 0.7255446775093857 & 0.1327096150655868\end{array}$

$\begin{array}{llll}0.5050566874696075 & 0.3943833889632163 & 0.1300028634265943\end{array}$

$\begin{array}{llll}0.3034189103753838 & 0.7267021302214298 & 0.1316645109929654\end{array}$

$\begin{array}{llll}0.8017534381010820 & 0.5583899793419994 & 0.1326339908176260\end{array}$

$\begin{array}{llll}0.9011276726557822 & 0.7262972824212954 & 0.1325842479317938\end{array}$

$\begin{array}{llll}0.9020591680570060 & 0.3920923014704559 & 0.1324660728875741\end{array}$

$\begin{array}{llll}0.7033290058235024 & 0.7255393019739182 & 0.1325704293589359\end{array}$

$\begin{array}{llll}0.3805919293102175 & 0.7566228850642550 & 0.2332054579686164\end{array}$

$\begin{array}{llll}0.7159532283354298 & 0.4636005908013142 & 0.2175907490744499\end{array}$

$\begin{array}{llll}0.3998941476909269 & 0.1485707076222014 & 0.2014851785328794\end{array}$

$\begin{array}{lllll}0.0691097218656657 & 0.4536425534700111 & 0.2073612869060603\end{array}$

$\begin{array}{llll}0.2142801818031278 & 0.1418246399348034 & 0.2077046542155960\end{array}$

$\begin{array}{llll}0.0726976878003315 & 0.2687464940534054 & 0.2115508812851930\end{array}$

$\begin{array}{llll}0.0636856690346319 & 0.6334729822618255 & 0.2054896602090677\end{array}$

$\begin{array}{llll}0.1942540397380282 & 0.7542827650519407 & 0.2311502094988951\end{array}$

$\begin{array}{lllll}0.5692268684654989 & 0.7687371735839157 & 0.2287191574990388\end{array}$

$\begin{array}{llll}0.7107770106832001 & 0.6443092390083389 & 0.2215286801042733\end{array}$

$\begin{array}{llll}0.7242131264535380 & 0.2806675919443510 & 0.2126086047786906\end{array}$

$\begin{array}{llll}0.5897748289461593 & 0.1482847172068360 & 0.2058830155166836\end{array}$

$\begin{array}{lllll}0.1905977906220756 & 0.3669577323578250 & 0.2012090702739369\end{array}$

$\begin{array}{llll}0.1477196467973011 & 0.2809014854387760 & 0.2060551711993428\end{array}$

$\begin{array}{llll}0.2189287623787290 & 0.2136591487629499 & 0.1996336623607797\end{array}$

$\begin{array}{llll}0.3076129917690563 & 0.2634252742709731 & 0.1966692580770280\end{array}$

$\begin{array}{llll}0.1408851008864832 & 0.4514502198897209 & 0.1952778903418903\end{array}$

$\begin{array}{llll}0.1907972684404317 & 0.5408652408089790 & 0.1963926506046217\end{array}$

$\begin{array}{llll}0.2970841029148250 & 0.6393168144057688 & 0.2205635996490035\end{array}$

$\begin{array}{lllll}0.2059895711268750 & 0.6842615870843661 & 0.2218204223164131\end{array}$

$\begin{array}{llll}0.1402833570080952 & 0.6244466809873230 & 0.2077306903547539\end{array}$

$\begin{array}{llll}0.3839868112886939 & 0.6835127067091977 & 0.2270803483640577\end{array}$

$\begin{array}{llll}0.4743049315797958 & 0.6450101518926558 & 0.2241460168227166\end{array}$

$\begin{array}{llll}0.5900334833050466 & 0.5434849272950213 & 0.2184439525152788\end{array}$

$\begin{array}{llll}0.6344691404963851 & 0.6323667767420560 & 0.2221743770894521\end{array}$

$\begin{array}{llll}0.5630736402686858 & 0.6949710932938624 & 0.2258064201407867\end{array}$

$\begin{array}{llll}0.6384720178528450 & 0.4606104435400226 & 0.2164311108127087\end{array}$

$\begin{array}{llll}0.5981357512699020 & 0.3736757774985539 & 0.2117183138895192\end{array}$

$\begin{array}{llll}0.4886149339050337 & 0.2673131445778932 & 0.2011445657071229\end{array}$

$\begin{array}{llll}0.3995187341440414 & 0.2215746819504311 & 0.1944435876279658\end{array}$

$\begin{array}{llll}0.5810356557384493 & 0.2207691187251305 & 0.1993269055208889\end{array}$

$\begin{array}{llll}0.6484403897782487 & 0.2902295117503596 & 0.2080528932399756\end{array}$

$\begin{array}{llll}0.2892239725061133 & 0.3567653101646082 & 0.1951057169372693\end{array}$

$\begin{array}{lllll}0.2864833917212690 & 0.5506325814511427 & 0.2084536228637772\end{array}$

$\begin{array}{llll}0.5001260350765280 & 0.3580281965120658 & 0.2067974178902569\end{array}$ 
$\begin{array}{llll}0.4922381782271490 & 0.5533432382634083 & 0.2192250477142553\end{array}$

$\begin{array}{llll}0.3979752845118507 & 0.4646578071407165 & 0.1953727086311880\end{array}$

\section{FeP-Pt(111) - Hhcp-45, vdW-DF-optB88:}

$\mathrm{Pt} \mathrm{H} \mathrm{C} \mathrm{N} \mathrm{Fe}$

1.00000000000000

$\begin{array}{lll}14.0790000000000006 & 0.0000000000000000 & 0.0000000000000000\end{array}$

$\begin{array}{lll}0.0000000000000000 & 14.6310000000000002 & 0.0000000000000000\end{array}$

$\begin{array}{llll}0.0000000000000000 & 0.0000000000000000 & 35.0000000000000000\end{array}$

$\mathrm{Pt} \quad \mathrm{H} \quad \mathrm{C} \quad \mathrm{N} \quad \mathrm{Fe}$

$\begin{array}{lllll}90 & 12 & 20 & 4 & 1\end{array}$

Direct

$0.0000000000000000 \quad 0.0000000000000000 \quad 0.0000000000000000$

$\begin{array}{llll}0.2000000000000028 & 0.0000000000000000 & 0.0000000000000000\end{array}$

$\begin{array}{llll}0.3999999999999986 & 0.0000000000000000 & 0.0000000000000000\end{array}$

$\begin{array}{llll}0.3000100000000003 & 0.1666799999999995 & 0.0000000000000000\end{array}$

$\begin{array}{llll}0.0000000000000000 & 0.3333399999999997 & 0.0000000000000000\end{array}$

$\begin{array}{lllll}0.2000000000000028 & 0.3333399999999997 & 0.0000000000000000\end{array}$

$\begin{array}{llll}0.1000099999999975 & 0.5000199999999992 & 0.0000000000000000\end{array}$

$\begin{array}{llll}0.1000099999999975 & 0.1666799999999995 & 0.0000000000000000\end{array}$

$\begin{array}{lllll}0.0000000000000000 & 0.6666999999999987 & 0.0000000000000000\end{array}$

$\begin{array}{lllll}0.6000099999999975 & 0.0000000000000000 & 0.0000000000000000\end{array}$

$\begin{array}{lll}0.8000200000000035 & 0.0000000000000000 & 0.0000000000000000\end{array}$

$\begin{array}{llll}0.7000099999999989 & 0.1666799999999995 & 0.0000000000000000\end{array}$

$\begin{array}{llll}0.3999999999999986 & 0.3333399999999997 & 0.0000000000000000\end{array}$

$\begin{array}{lllll}0.6000099999999975 & 0.3333399999999997 & 0.0000000000000000\end{array}$

$\begin{array}{llll}0.5000100000000032 & 0.5000199999999992 & 0.0000000000000000\end{array}$

$\begin{array}{llll}0.5000100000000032 & 0.1666799999999995 & 0.0000000000000000\end{array}$

$\begin{array}{llll}0.2000000000000028 & 0.6666999999999987 & 0.0000000000000000\end{array}$

$\begin{array}{llll}0.3999999999999986 & 0.6666999999999987 & 0.0000000000000000\end{array}$

$\begin{array}{llll}0.3000100000000003 & 0.8333599999999990 & 0.0000000000000000\end{array}$

$\begin{array}{lllll}0.3000100000000003 & 0.5000199999999992 & 0.0000000000000000\end{array}$

$\begin{array}{llll}0.1000099999999975 & 0.8333599999999990 & 0.0000000000000000\end{array}$

$\begin{array}{llll}0.8000200000000035 & 0.3333399999999997 & 0.0000000000000000\end{array}$

$\begin{array}{llll}0.9000199999999978 & 0.5000199999999992 & 0.0000000000000000\end{array}$

$\begin{array}{llll}0.9000199999999978 & 0.1666799999999995 & 0.0000000000000000\end{array}$

$\begin{array}{llll}0.6000099999999975 & 0.6666999999999987 & 0.0000000000000000\end{array}$

$\begin{array}{llll}0.8000200000000035 & 0.6666999999999987 & 0.0000000000000000\end{array}$

$\begin{array}{llll}0.7000099999999989 & 0.8333599999999990 & 0.0000000000000000\end{array}$

$\begin{array}{llll}0.7000099999999989 & 0.5000199999999992 & 0.0000000000000000\end{array}$

$\begin{array}{llll}0.5000100000000032 & 0.8333599999999990 & 0.0000000000000000\end{array}$

$\begin{array}{lllll}0.9000199999999978 & 0.8333599999999990 & 0.0000000000000000\end{array}$

$\begin{array}{llll}0.3000100000000003 & 0.9444400000000002 & 0.0656899999999965\end{array}$

$\begin{array}{llll}0.0000000000000000 & 0.1111199999999997 & 0.0656899999999965\end{array}$

$\begin{array}{llll}0.2000000000000028 & 0.1111199999999997 & 0.0656899999999965\end{array}$

$\begin{array}{lllll}0.1000099999999975 & 0.2777900000000031 & 0.0656899999999965\end{array}$

$\begin{array}{llll}0.1000099999999975 & 0.9444400000000002 & 0.0656899999999965\end{array}$ 
$\begin{array}{lll}0.0000000000000000 & 0.4444599999999994 & 0.0656899999999965\end{array}$

$\begin{array}{lllll}0.7000099999999989 & 0.9444400000000002 & 0.0656899999999965\end{array}$

$\begin{array}{lllll}0.3999999999999986 & 0.1111199999999997 & 0.0656899999999965\end{array}$

$\begin{array}{lllll}0.6000200000000007 & 0.1111199999999997 & 0.0656899999999965\end{array}$

$\begin{array}{lllll}0.5000100000000032 & 0.2777900000000031 & 0.0656899999999965\end{array}$

$\begin{array}{llll}0.5000100000000032 & 0.9444400000000002 & 0.0656899999999965\end{array}$

$\begin{array}{llll}0.2000000000000028 & 0.4444599999999994 & 0.0656899999999965\end{array}$

$\begin{array}{lllll}0.3999999999999986 & 0.4444599999999994 & 0.0656899999999965\end{array}$

$\begin{array}{llll}0.3000100000000003 & 0.6111399999999989 & 0.0656899999999965\end{array}$

$\begin{array}{llll}0.3000100000000003 & 0.2777900000000031 & 0.0656899999999965\end{array}$

$\begin{array}{llll}0.0000000000000000 & 0.7778100000000023 & 0.0656899999999965\end{array}$

$\begin{array}{lllll}0.2000000000000028 & 0.7778100000000023 & 0.0656899999999965\end{array}$

$\begin{array}{lllll}0.1000099999999975 & 0.6111399999999989 & 0.0656899999999965\end{array}$

$\begin{array}{llll}0.8000200000000035 & 0.1111199999999997 & 0.0656899999999965\end{array}$

$\begin{array}{lllll}0.9000199999999978 & 0.2777900000000031 & 0.0656899999999965\end{array}$

$\begin{array}{llll}0.9000199999999978 & 0.9444400000000002 & 0.0656899999999965\end{array}$

$\begin{array}{llll}0.6000200000000007 & 0.4444599999999994 & 0.0656899999999965\end{array}$

$\begin{array}{llll}0.8000200000000035 & 0.4444599999999994 & 0.0656899999999965\end{array}$

$\begin{array}{llll}0.7000099999999989 & 0.6111399999999989 & 0.0656899999999965\end{array}$

$\begin{array}{lllll}0.7000099999999989 & 0.2777900000000031 & 0.0656899999999965\end{array}$

$\begin{array}{llll}0.3999999999999986 & 0.7778100000000023 & 0.0656899999999965\end{array}$

$\begin{array}{lllll}0.6000200000000007 & 0.7778100000000023 & 0.0656899999999965\end{array}$

$\begin{array}{llll}0.5000100000000032 & 0.6111399999999989 & 0.0656899999999965\end{array}$

$\begin{array}{llll}0.8000200000000035 & 0.7778100000000023 & 0.0656899999999965\end{array}$

$\begin{array}{lllll}0.9000199999999978 & 0.6111399999999989 & 0.0656899999999965\end{array}$

$\begin{array}{llll}0.9991710625926640 & 0.8981812946967433 & 0.1325649448040624\end{array}$

$\begin{array}{llll}0.1975834646619890 & 0.9006781884908394 & 0.1324423644598272\end{array}$

$\begin{array}{llll}0.0995601443580163 & 0.0632632498572690 & 0.1327572123215590\end{array}$

$\begin{array}{llll}0.0000552120735492 & 0.2261201041967308 & 0.1317240344017657\end{array}$

$\begin{array}{llll}0.4006770010232520 & 0.9035766444083577 & 0.1322479075727574\end{array}$

$\begin{array}{llll}0.6033369995130006 & 0.9006300439976281 & 0.1324238986729598\end{array}$

$\begin{array}{lllll}0.5032585631331910 & 0.0636402830513115 & 0.1326021746172749\end{array}$

$\begin{array}{llll}0.2017340481139197 & 0.2291341025924145 & 0.1380385539126549\end{array}$

$\begin{array}{llll}0.4002646119514703 & 0.2258734710313196 & 0.1287326966710606\end{array}$

$\begin{array}{lllll}0.2991560388959873 & 0.3929285657205381 & 0.1292775620025829\end{array}$

$\begin{array}{llll}0.2978112785675657 & 0.0634313830873506 & 0.1325239963975966\end{array}$

$\begin{array}{llll}0.9997092798470946 & 0.5653921720711261 & 0.1315112198086782\end{array}$

$\begin{array}{llll}0.2000009195182137 & 0.5716547565858117 & 0.1339713363866908\end{array}$

$\begin{array}{llll}0.0983504615196080 & 0.7348091469643261 & 0.1315097048731398\end{array}$

$\begin{array}{llll}0.0942183919807869 & 0.3923205664085955 & 0.1353027202850108\end{array}$

$\begin{array}{llll}0.8017756977937500 & 0.8983171911188805 & 0.1326561485942505\end{array}$

$\begin{array}{llll}0.9005124902869922 & 0.0629457190586436 & 0.1326294161418066\end{array}$

$\begin{array}{llll}0.5993054982427640 & 0.2289854636171373 & 0.1380544164718233\end{array}$

$\begin{array}{llll}0.8004584420731884 & 0.2259600822034417 & 0.1316980029095882\end{array}$

$\begin{array}{llll}0.7063536646148593 & 0.3923642371196945 & 0.1352105581549754\end{array}$

$\begin{array}{llll}0.7015791196414236 & 0.0631635985248007 & 0.1328129872220345\end{array}$

$\begin{array}{llll}0.4006584429754245 & 0.5571022336275606 & 0.1294173203626497\end{array}$ 
$\begin{array}{llll}0.6007811809196869 & 0.5716790406575761 & 0.1339493344928398\end{array}$ $\begin{array}{llll}0.4993757646988239 & 0.7387160891694116 & 0.1341003546344703\end{array}$ $\begin{array}{llll}0.5023771070312326 & 0.3932844637140567 & 0.1293330564788435\end{array}$ $\begin{array}{llll}0.3010903853391773 & 0.7387248276958690 & 0.1341242259057936\end{array}$ $\begin{array}{llll}0.8008671340528313 & 0.5655791045312756 & 0.1316045924855320\end{array}$ $\begin{array}{llll}0.9005027224179261 & 0.7320231917044973 & 0.1326913303650866\end{array}$ $\begin{array}{llll}0.9001910032442001 & 0.3959508273196571 & 0.1307075931012015\end{array}$ $\begin{array}{llll}0.7026504347498312 & 0.7349935268501377 & 0.1315652258411146\end{array}$ $\begin{array}{llll}0.6288155695789521 & 0.6462558004056596 & 0.2035017325295629\end{array}$ $\begin{array}{llll}0.6307348627962391 & 0.2067351099517307 & 0.2113145156904477\end{array}$ $\begin{array}{llll}0.1718365803161924 & 0.2060943738075854 & 0.2113011125850578\end{array}$ $\begin{array}{llll}0.1720663994456150 & 0.6454792379302514 & 0.2037583463826430\end{array}$ $\begin{array}{llll}0.0371948505248623 & 0.3335765182200460 & 0.2065620269064598\end{array}$ $\begin{array}{llll}0.0383956022100159 & 0.5207527806324257 & 0.2078440108176584\end{array}$ $\begin{array}{llll}0.3079855757860057 & 0.7758509866670230 & 0.2082149770985518\end{array}$ $\begin{array}{llll}0.4926979678262328 & 0.7761218449922096 & 0.2081392316452131\end{array}$ $\begin{array}{lllll}0.7637439844561152 & 0.5221029134637977 & 0.2074855527285287\end{array}$ $\begin{array}{llll}0.7656838398668874 & 0.3347872145895344 & 0.2062018083096482\end{array}$ $\begin{array}{llll}0.4983959171415889 & 0.0809510547936555 & 0.2273146222527974\end{array}$ $\begin{array}{llll}0.3051639835914967 & 0.0800813216454964 & 0.2269586992091559\end{array}$ $\begin{array}{llll}0.1955593698462612 & 0.5007689480492942 & 0.2065832013889656\end{array}$ $\begin{array}{llll}0.0990424795470556 & 0.4748069813149475 & 0.2056924383411703\end{array}$ $\begin{array}{llll}0.0970118837580468 & 0.3783761963139183 & 0.2000303842075112\end{array}$ $\begin{array}{llll}0.1960323776280252 & 0.3493080481793831 & 0.2036400746331711\end{array}$ $\begin{array}{llll}0.2254363113122078 & 0.5927590960812932 & 0.1980243905368603\end{array}$ $\begin{array}{lllll}0.3208367029726530 & 0.6263303397414531 & 0.1977254531430986\end{array}$ $\begin{array}{lllll}0.4802508101256671 & 0.6265397525978504 & 0.1976535231650434\end{array}$ $\begin{array}{llll}0.4534351724901484 & 0.7224431193593146 & 0.1931490454423431\end{array}$ $\begin{array}{llll}0.3473342484083093 & 0.7222847418530625 & 0.1931860352036567\end{array}$ $\begin{array}{llll}0.5757503049552526 & 0.5931589419748404 & 0.1979252156558172\end{array}$ $\begin{array}{llll}0.6065170811282341 & 0.5013865100345354 & 0.2064973614067350\end{array}$ $\begin{array}{llll}0.6066938411204462 & 0.3499260826341777 & 0.2036898165460142\end{array}$ $\begin{array}{llll}0.7056003713807181 & 0.3792841047681338 & 0.1997214116503875\end{array}$ $\begin{array}{llll}0.7033022300668764 & 0.4759318277408107 & 0.2052639626761369\end{array}$ $\begin{array}{llll}0.5760114597458423 & 0.2558420129166858 & 0.2026106159122485\end{array}$ $\begin{array}{llll}0.4804160896772802 & 0.2281185208029275 & 0.2115898031845010\end{array}$ $\begin{array}{llll}0.3222492101463536 & 0.2274150101369016 & 0.2113966495858125\end{array}$ $\begin{array}{llll}0.2264792853338069 & 0.2551085402010947 & 0.2024232840932652\end{array}$ $\begin{array}{llll}0.3522112192012422 & 0.1374888246510102 & 0.2213556369680652\end{array}$ $\begin{array}{llll}0.4508410040876575 & 0.1378830019712954 & 0.2214627168625824\end{array}$ $\begin{array}{llll}0.2535867081089851 & 0.4237961405365563 & 0.2069056245623821\end{array}$ $\begin{array}{llll}0.4006130236941501 & 0.5705831299336976 & 0.1972318802150500\end{array}$ $\begin{array}{llll}0.4011853780274729 & 0.2827346336959429 & 0.2067315097914126\end{array}$ $\begin{array}{llll}0.5488873094461215 & 0.4242366820668233 & 0.2070531634004673\end{array}$ $\begin{array}{llll}0.4012015070447640 & 0.4196086817568760 & 0.1917582547730935\end{array}$ 


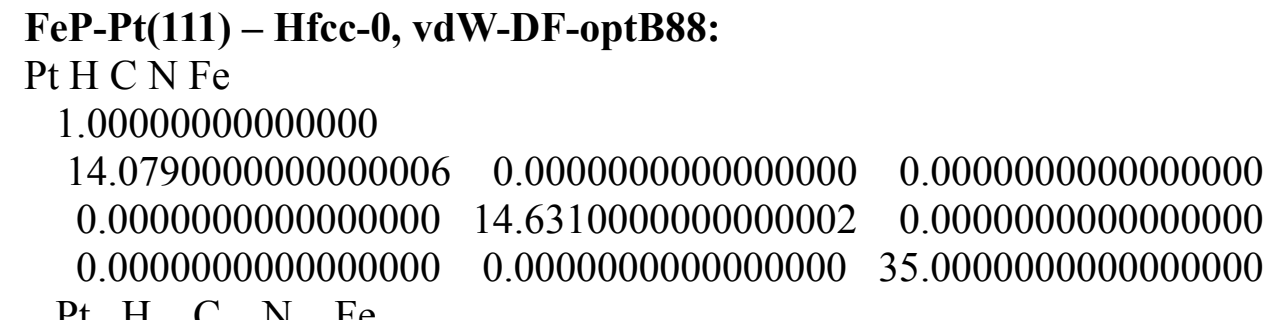

$\begin{array}{lllll}\mathrm{Pt} & \mathrm{H} & \mathrm{C} & \mathrm{N} & \mathrm{Fe}\end{array}$

$\begin{array}{lllll}90 & 12 & 20 & 4 & 1\end{array}$

Direct

$\begin{array}{llll}0.0000000000000000 & 0.0000000000000000 & 0.0000000000000000\end{array}$

$\begin{array}{lllll}0.2000000000000028 & 0.0000000000000000 & 0.0000000000000000\end{array}$

$\begin{array}{lllll}0.3999999999999986 & 0.0000000000000000 & 0.0000000000000000\end{array}$

$\begin{array}{lllll}0.3000100000000003 & 0.1666799999999995 & 0.0000000000000000\end{array}$

$\begin{array}{llll}0.0000000000000000 & 0.3333399999999997 & 0.0000000000000000\end{array}$

$\begin{array}{lllll}0.2000000000000028 & 0.3333399999999997 & 0.0000000000000000\end{array}$

$\begin{array}{llll}0.1000099999999975 & 0.5000199999999992 & 0.0000000000000000\end{array}$

$\begin{array}{llll}0.1000099999999975 & 0.1666799999999995 & 0.0000000000000000\end{array}$

$\begin{array}{lllll}0.0000000000000000 & 0.6666999999999987 & 0.0000000000000000\end{array}$

$\begin{array}{lllll}0.6000099999999975 & 0.0000000000000000 & 0.0000000000000000\end{array}$

$\begin{array}{lllll}0.8000200000000035 & 0.0000000000000000 & 0.0000000000000000\end{array}$

$\begin{array}{lllll}0.7000099999999989 & 0.1666799999999995 & 0.0000000000000000\end{array}$

$\begin{array}{lllll}0.3999999999999986 & 0.33333399999999997 & 0.0000000000000000\end{array}$

$\begin{array}{lllll}0.6000099999999975 & 0.3333399999999997 & 0.0000000000000000\end{array}$

$\begin{array}{llll}0.5000100000000032 & 0.5000199999999992 & 0.0000000000000000\end{array}$

$\begin{array}{llll}0.5000100000000032 & 0.1666799999999995 & 0.0000000000000000\end{array}$

$\begin{array}{llll}0.2000000000000028 & 0.6666999999999987 & 0.0000000000000000\end{array}$

$\begin{array}{lllll}0.3999999999999986 & 0.6666999999999987 & 0.0000000000000000\end{array}$

$\begin{array}{lllll}0.3000100000000003 & 0.8333599999999990 & 0.0000000000000000\end{array}$

$\begin{array}{lllll}0.3000100000000003 & 0.5000199999999992 & 0.0000000000000000\end{array}$

$\begin{array}{lllll}0.1000099999999975 & 0.8333599999999990 & 0.0000000000000000\end{array}$

$\begin{array}{lllll}0.8000200000000035 & 0.3333399999999997 & 0.0000000000000000\end{array}$

$\begin{array}{lllll}0.9000199999999978 & 0.5000199999999992 & 0.0000000000000000\end{array}$

$\begin{array}{llll}0.9000199999999978 & 0.1666799999999995 & 0.0000000000000000\end{array}$

$\begin{array}{llll}0.6000099999999975 & 0.6666999999999987 & 0.0000000000000000\end{array}$

$\begin{array}{lllll}0.8000200000000035 & 0.6666999999999987 & 0.0000000000000000\end{array}$

$\begin{array}{lllll}0.7000099999999989 & 0.8333599999999990 & 0.0000000000000000\end{array}$

$\begin{array}{lllll}0.7000099999999989 & 0.5000199999999992 & 0.0000000000000000\end{array}$

$\begin{array}{lllll}0.5000100000000032 & 0.8333599999999990 & 0.0000000000000000\end{array}$

$\begin{array}{lllll}0.9000199999999978 & 0.8333599999999990 & 0.0000000000000000\end{array}$

$\begin{array}{llllll}0.3000100000000003 & 0.9444400000000002 & 0.0656899999999965\end{array}$

$\begin{array}{lllll}0.0000000000000000 & 0.11111999999999997 & 0.06568999999999965\end{array}$

$\begin{array}{llll}0.2000000000000028 & 0.11111999999999997 & 0.0656899999999965\end{array}$

$\begin{array}{lllll}0.1000099999999975 & 0.2777900000000031 & 0.0656899999999965\end{array}$

$\begin{array}{lllll}0.1000099999999975 & 0.9444400000000002 & 0.0656899999999965\end{array}$

$\begin{array}{lllll}0.0000000000000000 & 0.4444599999999994 & 0.0656899999999965\end{array}$

$\begin{array}{lllll}0.7000099999999989 & 0.9444400000000002 & 0.0656899999999965\end{array}$

$\begin{array}{llll}0.3999999999999986 & 0.11111999999999997 & 0.0656899999999965\end{array}$ 
$\begin{array}{llll}0.6000200000000007 & 0.1111199999999997 & 0.0656899999999965\end{array}$

$\begin{array}{lllll}0.5000100000000032 & 0.2777900000000031 & 0.0656899999999965\end{array}$

$\begin{array}{llll}0.5000100000000032 & 0.9444400000000002 & 0.0656899999999965\end{array}$

$\begin{array}{llll}0.2000000000000028 & 0.4444599999999994 & 0.0656899999999965\end{array}$

$\begin{array}{llll}0.3999999999999986 & 0.4444599999999994 & 0.0656899999999965\end{array}$

$\begin{array}{llll}0.3000100000000003 & 0.6111399999999989 & 0.0656899999999965\end{array}$

$\begin{array}{lllll}0.3000100000000003 & 0.2777900000000031 & 0.0656899999999965\end{array}$

$\begin{array}{llll}0.0000000000000000 & 0.7778100000000023 & 0.0656899999999965\end{array}$

$\begin{array}{llll}0.2000000000000028 & 0.7778100000000023 & 0.0656899999999965\end{array}$

$\begin{array}{llll}0.1000099999999975 & 0.6111399999999989 & 0.0656899999999965\end{array}$

$\begin{array}{llll}0.8000200000000035 & 0.1111199999999997 & 0.0656899999999965\end{array}$

$\begin{array}{llll}0.9000199999999978 & 0.2777900000000031 & 0.0656899999999965\end{array}$

$\begin{array}{llll}0.9000199999999978 & 0.9444400000000002 & 0.0656899999999965\end{array}$

$\begin{array}{llll}0.6000200000000007 & 0.4444599999999994 & 0.0656899999999965\end{array}$

$\begin{array}{lll}0.8000200000000035 & 0.4444599999999994 & 0.0656899999999965\end{array}$

$\begin{array}{llll}0.7000099999999989 & 0.6111399999999989 & 0.0656899999999965\end{array}$

$\begin{array}{lllll}0.7000099999999989 & 0.2777900000000031 & 0.0656899999999965\end{array}$

$\begin{array}{llll}0.3999999999999986 & 0.7778100000000023 & 0.0656899999999965\end{array}$

$\begin{array}{llll}0.6000200000000007 & 0.7778100000000023 & 0.0656899999999965\end{array}$

$\begin{array}{llll}0.5000100000000032 & 0.6111399999999989 & 0.0656899999999965\end{array}$

$\begin{array}{lllll}0.8000200000000035 & 0.7778100000000023 & 0.0656899999999965\end{array}$

$\begin{array}{lllll}0.9000199999999978 & 0.6111399999999989 & 0.0656899999999965\end{array}$

$\begin{array}{llll}0.0000009550361312 & 0.8904244527516434 & 0.1324998998823335\end{array}$

$\begin{array}{llll}0.1972557331899918 & 0.8952377460765661 & 0.1321876744340947\end{array}$

$\begin{array}{llll}0.0987898435293162 & 0.0562429548963547 & 0.1327599300281682\end{array}$

$\begin{array}{llll}0.9998325239626727 & 0.2201327694950712 & 0.1329513906241105\end{array}$

$\begin{array}{llll}0.3995230946238169 & 0.9014118017021843 & 0.1317703518731431\end{array}$

$\begin{array}{llll}0.6004532832129366 & 0.9013283591522397 & 0.1317413505629656\end{array}$

$\begin{array}{llll}0.4999944127949055 & 0.0614757157047106 & 0.1335431602965847\end{array}$

$\begin{array}{llll}0.1975228843146931 & 0.2196244536832262 & 0.1323631908992311\end{array}$

$\begin{array}{llll}0.3993906679321950 & 0.2225474831591468 & 0.1316684257928714\end{array}$

$\begin{array}{llll}0.2967713030964707 & 0.3836339230823253 & 0.1337406763301513\end{array}$

$\begin{array}{llll}0.2989129949645709 & 0.0594713286860502 & 0.1328204178781558\end{array}$

$\begin{array}{llll}0.9997291910555268 & 0.5554797986768388 & 0.1320744726022748\end{array}$

$\begin{array}{llll}0.1990304995430710 & 0.5464344599468894 & 0.1364855411245249\end{array}$

$\begin{array}{llll}0.0993758395019330 & 0.7218927016652675 & 0.1313548483074473\end{array}$

$\begin{array}{lllll}0.0959626851337477 & 0.3850754612541236 & 0.1313929094381029\end{array}$

$\begin{array}{llll}0.8027055265599353 & 0.8949657542367542 & 0.1321547619562112\end{array}$

$\begin{array}{llll}0.9012093729792131 & 0.0562671585023937 & 0.1327558869503664\end{array}$

$\begin{array}{llll}0.6004270122000686 & 0.2224714044736210 & 0.1316110132232680\end{array}$

$\begin{array}{llll}0.8023925774141207 & 0.2196625358790939 & 0.1323531229410440\end{array}$

$\begin{array}{llll}0.7026090738775279 & 0.3833466751975979 & 0.1337275922970535\end{array}$

$\begin{array}{llll}0.7011086159047955 & 0.0594027219584527 & 0.1328105744607624\end{array}$

$\begin{array}{llll}0.3978199662008822 & 0.5600737805455742 & 0.1299970944515929\end{array}$

$\begin{array}{llll}0.6004874588337832 & 0.5592251661119273 & 0.1298250681757986\end{array}$

$\begin{array}{llll}0.4998668938488348 & 0.7370706964129228 & 0.1308782984606072\end{array}$

$\begin{array}{llll}0.4990481078357076 & 0.3886446333832153 & 0.1298976228396488\end{array}$ 
$\begin{array}{llll}0.2969829600756650 & 0.7365171597788017 & 0.1339843810521012\end{array}$ $\begin{array}{llll}0.7999271548343775 & 0.5464945413135078 & 0.1365024251696391\end{array}$ $\begin{array}{llll}0.9007433168251714 & 0.7218919155117128 & 0.1312603282012595\end{array}$ $\begin{array}{llll}0.9034459210685638 & 0.3853569033217733 & 0.1315727987659584\end{array}$ $\begin{array}{llll}0.7029607386166967 & 0.7360930822450367 & 0.1339176482024504\end{array}$ $\begin{array}{llll}0.4994889531137767 & 0.8029315227232597 & 0.2021654842744553\end{array}$ $\begin{array}{llll}0.8257615867127702 & 0.4947940182553965 & 0.2082575657814623\end{array}$ $\begin{array}{llll}0.4986050740817952 & 0.2111595414988534 & 0.2404263174295236\end{array}$ $\begin{array}{llll}0.1720995798412792 & 0.4952274779985434 & 0.2082076973979475\end{array}$ $\begin{array}{lllll}0.3111896166553875 & 0.1996602893912614 & 0.2343797868161245\end{array}$ $\begin{array}{llll}0.1741623703637749 & 0.3112728119433190 & 0.2075144907375475\end{array}$ $\begin{array}{llll}0.1742925278878077 & 0.6818765144245302 & 0.2133870106652437\end{array}$ $\begin{array}{llll}0.3144101577032257 & 0.8096366592276959 & 0.2079244506010204\end{array}$ $\begin{array}{llll}0.6831584125656904 & 0.8089933171400358 & 0.2073827849692784\end{array}$ $\begin{array}{llll}0.8235193106922175 & 0.6812887648085575 & 0.2130500905689788\end{array}$ $\begin{array}{llll}0.8231167767672608 & 0.3098388926831069 & 0.2078134340405628\end{array}$ $\begin{array}{llll}0.6851787069711823 & 0.1986655365730025 & 0.2342689419605609\end{array}$ $\begin{array}{llll}0.2956649799570190 & 0.4100426099156983 & 0.1969472236981527\end{array}$ $\begin{array}{llll}0.2500776589494150 & 0.3247427183370040 & 0.2094645599389364\end{array}$ $\begin{array}{llll}0.3193388087139562 & 0.2693684440671867 & 0.2240698018549779\end{array}$ $\begin{array}{llll}0.4085113581789985 & 0.3186785020405564 & 0.2217992684290445\end{array}$ $\begin{array}{llll}0.2432297998688827 & 0.4985514351689356 & 0.1955807895574069\end{array}$ $\begin{array}{llll}0.2914760078535620 & 0.5843171512599026 & 0.2007744050231174\end{array}$ $\begin{array}{llll}0.4072047237487467 & 0.6890194712259357 & 0.1941392368146605\end{array}$ $\begin{array}{llll}0.3186228488880730 & 0.7381596020388528 & 0.1992411698243304\end{array}$ $\begin{array}{lllll}0.2486928656147468 & 0.6699906291988752 & 0.2066406061176189\end{array}$ $\begin{array}{lllll}0.4990770295719487 & 0.7317094539735239 & 0.1924240052501339\end{array}$ $\begin{array}{llll}0.5906166991681281 & 0.6884645136899896 & 0.1937546539270870\end{array}$ $\begin{array}{llll}0.7062439552819049 & 0.5837482929043405 & 0.2006921380019205\end{array}$ $\begin{array}{llll}0.7490671922434325 & 0.6694464586934961 & 0.2063651751859859\end{array}$ $\begin{array}{llll}0.6792864692183112 & 0.7375686227585716 & 0.1985878764696062\end{array}$ $\begin{array}{llll}0.7546039882243427 & 0.4978841974090876 & 0.1956421839986845\end{array}$ $\begin{array}{llll}0.7022946679981733 & 0.4092912444266152 & 0.1970143728727335\end{array}$ $\begin{array}{llll}0.5887720592369234 & 0.3184040788566905 & 0.2217601890738605\end{array}$ $\begin{array}{llll}0.4986558603049124 & 0.2812770470986718 & 0.2297242219077749\end{array}$ $\begin{array}{llll}0.6774905703602982 & 0.2685275457684943 & 0.2241256378390271\end{array}$ $\begin{array}{llll}0.7472324912682151 & 0.3236822328616148 & 0.2097429436656953\end{array}$ $\begin{array}{llll}0.3921322981493542 & 0.4046908184890727 & 0.2079678557475534\end{array}$ $\begin{array}{llll}0.3893427421804874 & 0.5956234330853835 & 0.1926074454955493\end{array}$ $\begin{array}{llll}0.6056234857585964 & 0.4043095463592994 & 0.2078756932118413\end{array}$ $\begin{array}{llll}0.6083504289848706 & 0.5949709892653309 & 0.1924925450674384\end{array}$ $\begin{array}{lllll}0.4988276932265326 & 0.4901588761793639 & 0.1929608228130739\end{array}$

\section{FeP-Pt(111) - Hfcc-45, vdW-DF-optB88:}

$\mathrm{Pt} \mathrm{H} \mathrm{C} \mathrm{N} \mathrm{Fe}$ 1.00000000000000
14.0790000000000006
0.0000000000000000
0.0000000000000000 


$\begin{array}{rrrr}0.0000000000000000 & 14.6310000000000002 & 0.0000000000000000 \\ 0.0000000000000000 & 0.0000000000000000 & 35.0000000000000000\end{array}$

$\mathrm{Pt} \mathrm{H} \quad \mathrm{C} \quad \mathrm{N}$ Fe

Direct

$\begin{array}{lllll}90 & 12 & 20 & 4 & 1\end{array}$

$0.0000000000000000 \quad 0.0000000000000000 \quad 0.0000000000000000$

$\begin{array}{llll}0.2000000000000028 & 0.0000000000000000 & 0.0000000000000000\end{array}$

$\begin{array}{llll}0.3999999999999986 & 0.0000000000000000 & 0.0000000000000000\end{array}$

$\begin{array}{llll}0.3000100000000003 & 0.1666799999999995 & 0.0000000000000000\end{array}$

$\begin{array}{llll}0.0000000000000000 & 0.3333399999999997 & 0.0000000000000000\end{array}$

$\begin{array}{llll}0.2000000000000028 & 0.3333399999999997 & 0.0000000000000000\end{array}$

$\begin{array}{llll}0.1000099999999975 & 0.5000199999999992 & 0.0000000000000000\end{array}$

$\begin{array}{llll}0.1000099999999975 & 0.1666799999999995 & 0.0000000000000000\end{array}$

$\begin{array}{llll}0.0000000000000000 & 0.6666999999999987 & 0.0000000000000000\end{array}$

$\begin{array}{llll}0.6000099999999975 & 0.0000000000000000 & 0.0000000000000000\end{array}$

$\begin{array}{llll}0.8000200000000035 & 0.0000000000000000 & 0.0000000000000000\end{array}$

$\begin{array}{llll}0.7000099999999989 & 0.1666799999999995 & 0.0000000000000000\end{array}$

$\begin{array}{llll}0.3999999999999986 & 0.3333399999999997 & 0.0000000000000000\end{array}$

$\begin{array}{llll}0.6000099999999975 & 0.3333399999999997 & 0.0000000000000000\end{array}$

$\begin{array}{llll}0.5000100000000032 & 0.5000199999999992 & 0.0000000000000000\end{array}$

$\begin{array}{llll}0.5000100000000032 & 0.1666799999999995 & 0.0000000000000000\end{array}$

$\begin{array}{lllll}0.2000000000000028 & 0.6666999999999987 & 0.0000000000000000\end{array}$

$\begin{array}{llll}0.3999999999999986 & 0.6666999999999987 & 0.0000000000000000\end{array}$

$\begin{array}{llll}0.3000100000000003 & 0.8333599999999990 & 0.0000000000000000\end{array}$

$\begin{array}{llll}0.3000100000000003 & 0.5000199999999992 & 0.0000000000000000\end{array}$

$\begin{array}{llllll}0.1000099999999975 & 0.8333599999999990 & 0.0000000000000000\end{array}$

$\begin{array}{lllll}0.8000200000000035 & 0.3333399999999997 & 0.0000000000000000\end{array}$

$\begin{array}{llll}0.9000199999999978 & 0.5000199999999992 & 0.0000000000000000\end{array}$

$\begin{array}{llll}0.9000199999999978 & 0.1666799999999995 & 0.0000000000000000\end{array}$

$\begin{array}{llll}0.6000099999999975 & 0.6666999999999987 & 0.0000000000000000\end{array}$

$\begin{array}{lllll}0.8000200000000035 & 0.6666999999999987 & 0.0000000000000000\end{array}$

$\begin{array}{lllll}0.7000099999999989 & 0.8333599999999990 & 0.0000000000000000\end{array}$

$\begin{array}{llll}0.7000099999999989 & 0.5000199999999992 & 0.0000000000000000\end{array}$

$\begin{array}{llll}0.5000100000000032 & 0.8333599999999990 & 0.0000000000000000\end{array}$

$\begin{array}{llll}0.9000199999999978 & 0.8333599999999990 & 0.0000000000000000\end{array}$

$\begin{array}{llll}0.3000100000000003 & 0.9444400000000002 & 0.0656899999999965\end{array}$

$\begin{array}{lllll}0.0000000000000000 & 0.1111199999999997 & 0.0656899999999965\end{array}$

$\begin{array}{lllll}0.2000000000000028 & 0.1111199999999997 & 0.0656899999999965\end{array}$

$\begin{array}{lllll}0.1000099999999975 & 0.2777900000000031 & 0.0656899999999965\end{array}$

$\begin{array}{llll}0.1000099999999975 & 0.9444400000000002 & 0.0656899999999965\end{array}$

$\begin{array}{lllll}0.0000000000000000 & 0.4444599999999994 & 0.0656899999999965\end{array}$

$\begin{array}{llll}0.7000099999999989 & 0.9444400000000002 & 0.0656899999999965\end{array}$

$\begin{array}{llll}0.3999999999999986 & 0.1111199999999997 & 0.0656899999999965\end{array}$

$\begin{array}{llll}0.6000200000000007 & 0.1111199999999997 & 0.0656899999999965\end{array}$

$\begin{array}{llll}0.5000100000000032 & 0.2777900000000031 & 0.0656899999999965\end{array}$

$\begin{array}{llll}0.5000100000000032 & 0.9444400000000002 & 0.0656899999999965\end{array}$

$\begin{array}{llll}0.2000000000000028 & 0.4444599999999994 & 0.0656899999999965\end{array}$ 
$\begin{array}{lll}0.3999999999999986 & 0.4444599999999994 & 0.0656899999999965\end{array}$ $\begin{array}{llll}0.3000100000000003 & 0.6111399999999989 & 0.0656899999999965\end{array}$ $\begin{array}{lllll}0.3000100000000003 & 0.2777900000000031 & 0.0656899999999965\end{array}$ $\begin{array}{llll}0.0000000000000000 & 0.7778100000000023 & 0.0656899999999965\end{array}$ $\begin{array}{llll}0.2000000000000028 & 0.7778100000000023 & 0.0656899999999965\end{array}$ $\begin{array}{llll}0.1000099999999975 & 0.6111399999999989 & 0.0656899999999965\end{array}$ $\begin{array}{lllll}0.8000200000000035 & 0.1111199999999997 & 0.0656899999999965\end{array}$ $\begin{array}{lllll}0.9000199999999978 & 0.2777900000000031 & 0.0656899999999965\end{array}$ $\begin{array}{llll}0.9000199999999978 & 0.9444400000000002 & 0.0656899999999965\end{array}$ $\begin{array}{llll}0.6000200000000007 & 0.4444599999999994 & 0.0656899999999965\end{array}$ $\begin{array}{llll}0.8000200000000035 & 0.4444599999999994 & 0.0656899999999965\end{array}$ $\begin{array}{llll}0.7000099999999989 & 0.6111399999999989 & 0.0656899999999965\end{array}$ $\begin{array}{lllll}0.7000099999999989 & 0.2777900000000031 & 0.0656899999999965\end{array}$ $\begin{array}{llll}0.3999999999999986 & 0.7778100000000023 & 0.0656899999999965\end{array}$ $\begin{array}{llll}0.6000200000000007 & 0.7778100000000023 & 0.0656899999999965\end{array}$ $\begin{array}{lllll}0.5000100000000032 & 0.6111399999999989 & 0.0656899999999965\end{array}$ $\begin{array}{lllll}0.8000200000000035 & 0.7778100000000023 & 0.0656899999999965\end{array}$ $\begin{array}{lllll}0.9000199999999978 & 0.6111399999999989 & 0.0656899999999965\end{array}$ $\begin{array}{llll}0.9989669906333936 & 0.8889573179877041 & 0.1324711502898664\end{array}$ $\begin{array}{llll}0.1987526018905709 & 0.8883426372937041 & 0.1322656610766448\end{array}$ $\begin{array}{lllll}0.0978775065206851 & 0.0536718163086596 & 0.1326623068382684\end{array}$ $\begin{array}{llll}0.9989853198696560 & 0.2188422234361506 & 0.1328100080743436\end{array}$ $\begin{array}{llll}0.3981853057368088 & 0.8890850656169619 & 0.1323771301600829\end{array}$ $\begin{array}{llll}0.5998557366188798 & 0.8898687943935412 & 0.1322274249730664\end{array}$ $\begin{array}{llll}0.4989481107295088 & 0.0511395231793651 & 0.1317249922560580\end{array}$ $\begin{array}{llll}0.1962284345450639 & 0.2161715166893003 & 0.1318848162270289\end{array}$ $\begin{array}{llll}0.4001544580239147 & 0.2140342314246798 & 0.1339355637179115\end{array}$ $\begin{array}{llll}0.2995667218598924 & 0.3782941825168920 & 0.1340299338273255\end{array}$ $\begin{array}{llll}0.2965905675088473 & 0.0513322903952851 & 0.1326104845687844\end{array}$ $\begin{array}{llll}0.9990987747117840 & 0.5564349232460641 & 0.1304935671220272\end{array}$ $\begin{array}{llll}0.1940053232816731 & 0.5577405503946761 & 0.1352856336162898\end{array}$ $\begin{array}{llll}0.0988391253257064 & 0.7260031585335173 & 0.1316752103477996\end{array}$ $\begin{array}{llll}0.0996881197395715 & 0.3851553846072421 & 0.1309732258839674\end{array}$ $\begin{array}{lllll}0.7992091189020798 & 0.8886211886629087 & 0.1322107742060377\end{array}$ $\begin{array}{llll}0.9004626540400265 & 0.0533638541880209 & 0.1327075420266577\end{array}$ $\begin{array}{llll}0.5983546513514564 & 0.2143642459393527 & 0.1340246274596371\end{array}$ $\begin{array}{llll}0.8026030348716716 & 0.2164235497774893 & 0.1319768490210089\end{array}$ $\begin{array}{llll}0.6998567506212409 & 0.3783426512377241 & 0.1340832926877455\end{array}$ $\begin{array}{llll}0.7011852156154663 & 0.0520613751539041 & 0.1323964237946255\end{array}$ $\begin{array}{llll}0.3964932727669632 & 0.5572113449171157 & 0.1293217827215146\end{array}$ $\begin{array}{llll}0.6027492070582892 & 0.5569472722271767 & 0.1294494414355397\end{array}$ $\begin{array}{lllll}0.4996049766034683 & 0.7260355887837501 & 0.1290064118338966\end{array}$ $\begin{array}{llll}0.4998131237832800 & 0.3942444685906610 & 0.1291461646809221\end{array}$ $\begin{array}{llll}0.3011315655563280 & 0.7222863624646743 & 0.1376860603159626\end{array}$ $\begin{array}{llll}0.8047291165865860 & 0.5578883854828733 & 0.1352962520452110\end{array}$ $\begin{array}{lllll}0.8998107435606099 & 0.7260646723617574 & 0.1316929918939927\end{array}$ $\begin{array}{llll}0.8999467648098411 & 0.3851302311713793 & 0.1310254877814985\end{array}$ 
$\begin{array}{llll}0.6967737930231980 & 0.7228930743641766 & 0.1373340636973318\end{array}$

$\begin{array}{lllll}0.2678030260004592 & 0.7484299865437626 & 0.2109265259112764\end{array}$

$\begin{array}{llll}0.7268858280056711 & 0.7496705544603824 & 0.2109307462512247\end{array}$

$\begin{array}{llll}0.7279653635074679 & 0.3097858747590210 & 0.2041225785861247\end{array}$

$\begin{array}{llll}0.2707164797204408 & 0.3091327992368285 & 0.2040673859512052\end{array}$

$\begin{array}{llll}0.5910253778872835 & 0.1789512378106696 & 0.2081810038580978\end{array}$

$\begin{array}{llll}0.4069333947336450 & 0.1792121588187200 & 0.2081355501050720\end{array}$

$\begin{array}{llll}0.1360795355860150 & 0.4330864578545468 & 0.2075875566340265\end{array}$

$\begin{array}{llll}0.1338409610713924 & 0.6202674623358907 & 0.2061160550256068\end{array}$

$\begin{array}{llll}0.4005375507449697 & 0.8743794508003586 & 0.2277936805989484\end{array}$

$\begin{array}{llll}0.5940202211757372 & 0.8745529227944976 & 0.2278134804900656\end{array}$

$\begin{array}{llll}0.8621279768871362 & 0.6229896167531035 & 0.2061443301993621\end{array}$

$\begin{array}{llll}0.8622946960161690 & 0.4357885285607351 & 0.2074147184721216\end{array}$

$\begin{array}{llll}0.4192858211356620 & 0.3286085194491584 & 0.1973833884544897\end{array}$

$\begin{array}{llll}0.4459100351217875 & 0.2326457221773467 & 0.1928421335054578\end{array}$

$\begin{array}{lllll}0.5522204438832301 & 0.2324208111149661 & 0.1928440601306710\end{array}$

$\begin{array}{lllll}0.5791638020617300 & 0.3285341588827081 & 0.1973651000275973\end{array}$

$\begin{array}{llll}0.3236213816038616 & 0.3620690933005649 & 0.1979088169996251\end{array}$

$\begin{array}{llll}0.2932842560635006 & 0.4539130137329979 & 0.2065759419084826\end{array}$

$\begin{array}{lllll}0.2928804349714707 & 0.6053774707002841 & 0.2038357736101951\end{array}$

$\begin{array}{llll}0.1941545254986423 & 0.5757278485595364 & 0.2001183629171862\end{array}$

$\begin{array}{llll}0.1964604435144786 & 0.4792799637024634 & 0.2054061992220011\end{array}$

$\begin{array}{lllll}0.3228628100026248 & 0.6996285380991181 & 0.2023194900262408\end{array}$

$\begin{array}{llll}0.4184367304373616 & 0.7281035332815436 & 0.2111712122141052\end{array}$

$\begin{array}{llll}0.5766004436602792 & 0.7280622221744142 & 0.2111840048250226\end{array}$

$\begin{array}{llll}0.5467873910700153 & 0.8175377549800977 & 0.2217380607521732\end{array}$

$\begin{array}{llll}0.4480458828188309 & 0.8175601771672295 & 0.2217377549001044\end{array}$

$\begin{array}{llll}0.6723424456971436 & 0.7001274540975562 & 0.2025533276490020\end{array}$

$\begin{array}{llll}0.7033997495919648 & 0.6063058549103277 & 0.2038208251919910\end{array}$

$\begin{array}{llll}0.7046565209904393 & 0.4546509279288399 & 0.2062192850947582\end{array}$

$\begin{array}{llll}0.6748215940367952 & 0.3624089337530157 & 0.1978349196371502\end{array}$

$\begin{array}{llll}0.8013097324898503 & 0.4812227353133583 & 0.2051187566760384\end{array}$

$\begin{array}{llll}0.8024184646317017 & 0.5778038142481121 & 0.2000453496137007\end{array}$

$\begin{array}{llll}0.4992136527399787 & 0.3843695722501702 & 0.1970470820176321\end{array}$

$\begin{array}{lllll}0.3507920761971687 & 0.5310301195036439 & 0.2070268625996332\end{array}$

$\begin{array}{llll}0.6462932474154700 & 0.5312453348298858 & 0.2067144319175239\end{array}$

$\begin{array}{llll}0.4974926281203630 & 0.6732149773335652 & 0.2058413530942184\end{array}$

$\begin{array}{lllll}0.4981317533050306 & 0.5354080576368309 & 0.1916357199423218\end{array}$

FeP-Pt(111) - B-0, vdW-DF-optB88:

$\mathrm{Pt} \mathrm{H} \mathrm{C} \mathrm{N} \mathrm{Fe}$

1.00000000000000

$\begin{array}{lll}14.0790000000000006 & 0.0000000000000000 & 0.0000000000000000\end{array}$

$\begin{array}{llll}0.0000000000000000 & 14.6310000000000002 & 0.0000000000000000\end{array}$

$\begin{array}{llll}0.0000000000000000 & 0.0000000000000000 & 35.0000000000000000\end{array}$

$\mathrm{Pt} \mathrm{H} \quad \mathrm{C} \quad \mathrm{N}$ Fe

$\begin{array}{lllll}90 & 12 & 20 & 4 & 1\end{array}$ 
Direct

$0.0000000000000000 \quad 0.0000000000000000 \quad 0.0000000000000000$

$\begin{array}{llll}0.2000000000000028 & 0.0000000000000000 & 0.0000000000000000\end{array}$

$\begin{array}{llll}0.3999999999999986 & 0.0000000000000000 & 0.0000000000000000\end{array}$

$\begin{array}{llll}0.3000100000000003 & 0.1666799999999995 & 0.0000000000000000\end{array}$

$\begin{array}{llll}0.0000000000000000 & 0.3333399999999997 & 0.0000000000000000\end{array}$

$\begin{array}{lllll}0.2000000000000028 & 0.3333399999999997 & 0.0000000000000000\end{array}$

$\begin{array}{llll}0.1000099999999975 & 0.5000199999999992 & 0.0000000000000000\end{array}$

$\begin{array}{lllll}0.1000099999999975 & 0.1666799999999995 & 0.0000000000000000\end{array}$

$\begin{array}{llll}0.0000000000000000 & 0.6666999999999987 & 0.0000000000000000\end{array}$

$\begin{array}{llll}0.6000099999999975 & 0.0000000000000000 & 0.0000000000000000\end{array}$

$\begin{array}{llll}0.8000200000000035 & 0.0000000000000000 & 0.0000000000000000\end{array}$

$\begin{array}{llll}0.7000099999999989 & 0.1666799999999995 & 0.0000000000000000\end{array}$

$\begin{array}{lllll}0.3999999999999986 & 0.3333399999999997 & 0.0000000000000000\end{array}$

$\begin{array}{llll}0.6000099999999975 & 0.3333399999999997 & 0.0000000000000000\end{array}$

$\begin{array}{llll}0.5000100000000032 & 0.5000199999999992 & 0.0000000000000000\end{array}$

$\begin{array}{llll}0.5000100000000032 & 0.1666799999999995 & 0.0000000000000000\end{array}$

$\begin{array}{llll}0.2000000000000028 & 0.6666999999999987 & 0.0000000000000000\end{array}$

$\begin{array}{llll}0.3999999999999986 & 0.6666999999999987 & 0.0000000000000000\end{array}$

$\begin{array}{llll}0.3000100000000003 & 0.8333599999999990 & 0.0000000000000000\end{array}$

$\begin{array}{llll}0.3000100000000003 & 0.5000199999999992 & 0.0000000000000000\end{array}$

$\begin{array}{lllll}0.1000099999999975 & 0.8333599999999990 & 0.0000000000000000\end{array}$

$\begin{array}{llll}0.8000200000000035 & 0.3333399999999997 & 0.0000000000000000\end{array}$

$\begin{array}{llll}0.9000199999999978 & 0.5000199999999992 & 0.0000000000000000\end{array}$

$\begin{array}{llll}0.9000199999999978 & 0.1666799999999995 & 0.0000000000000000\end{array}$

$\begin{array}{lllll}0.6000099999999975 & 0.6666999999999987 & 0.0000000000000000\end{array}$

$\begin{array}{lllll}0.8000200000000035 & 0.6666999999999987 & 0.0000000000000000\end{array}$

$\begin{array}{lllll}0.7000099999999989 & 0.8333599999999990 & 0.0000000000000000\end{array}$

$\begin{array}{llll}0.7000099999999989 & 0.5000199999999992 & 0.0000000000000000\end{array}$

$\begin{array}{llll}0.5000100000000032 & 0.8333599999999990 & 0.0000000000000000\end{array}$

$\begin{array}{lllll}0.9000199999999978 & 0.8333599999999990 & 0.0000000000000000\end{array}$

$\begin{array}{llll}0.3000100000000003 & 0.9444400000000002 & 0.0656899999999965\end{array}$

$\begin{array}{lllll}0.0000000000000000 & 0.1111199999999997 & 0.0656899999999965\end{array}$

$\begin{array}{lllll}0.2000000000000028 & 0.1111199999999997 & 0.0656899999999965\end{array}$

$\begin{array}{lllll}0.1000099999999975 & 0.2777900000000031 & 0.0656899999999965\end{array}$

$\begin{array}{lllll}0.1000099999999975 & 0.9444400000000002 & 0.0656899999999965\end{array}$

$\begin{array}{llll}0.0000000000000000 & 0.4444599999999994 & 0.0656899999999965\end{array}$

$\begin{array}{llll}0.7000099999999989 & 0.9444400000000002 & 0.0656899999999965\end{array}$

$\begin{array}{llll}0.3999999999999986 & 0.1111199999999997 & 0.0656899999999965\end{array}$

$\begin{array}{lllll}0.6000200000000007 & 0.1111199999999997 & 0.0656899999999965\end{array}$

$\begin{array}{lllll}0.5000100000000032 & 0.2777900000000031 & 0.0656899999999965\end{array}$

$\begin{array}{llll}0.5000100000000032 & 0.9444400000000002 & 0.0656899999999965\end{array}$

$\begin{array}{llll}0.2000000000000028 & 0.4444599999999994 & 0.0656899999999965\end{array}$

$\begin{array}{llll}0.3999999999999986 & 0.4444599999999994 & 0.0656899999999965\end{array}$

$\begin{array}{lllll}0.3000100000000003 & 0.6111399999999989 & 0.0656899999999965\end{array}$

$\begin{array}{lllll}0.3000100000000003 & 0.2777900000000031 & 0.0656899999999965\end{array}$

$\begin{array}{llll}0.0000000000000000 & 0.7778100000000023 & 0.0656899999999965\end{array}$ 
$\begin{array}{llll}0.2000000000000028 & 0.7778100000000023 & 0.0656899999999965\end{array}$

$\begin{array}{lllll}0.1000099999999975 & 0.6111399999999989 & 0.0656899999999965\end{array}$

$\begin{array}{lllll}0.8000200000000035 & 0.1111199999999997 & 0.0656899999999965\end{array}$

$\begin{array}{lllll}0.9000199999999978 & 0.2777900000000031 & 0.0656899999999965\end{array}$

$\begin{array}{llll}0.9000199999999978 & 0.9444400000000002 & 0.0656899999999965\end{array}$

$\begin{array}{llll}0.6000200000000007 & 0.4444599999999994 & 0.0656899999999965\end{array}$

$\begin{array}{llll}0.8000200000000035 & 0.4444599999999994 & 0.0656899999999965\end{array}$

$\begin{array}{lllll}0.7000099999999989 & 0.6111399999999989 & 0.0656899999999965\end{array}$

$\begin{array}{lllll}0.7000099999999989 & 0.2777900000000031 & 0.0656899999999965\end{array}$

$\begin{array}{llll}0.3999999999999986 & 0.7778100000000023 & 0.0656899999999965\end{array}$

$\begin{array}{llll}0.6000200000000007 & 0.7778100000000023 & 0.0656899999999965\end{array}$

$\begin{array}{llll}0.5000100000000032 & 0.6111399999999989 & 0.0656899999999965\end{array}$

$\begin{array}{llll}0.8000200000000035 & 0.7778100000000023 & 0.0656899999999965\end{array}$

$\begin{array}{llll}0.9000199999999978 & 0.6111399999999989 & 0.0656899999999965\end{array}$

$\begin{array}{llll}0.0004635195840283 & 0.8905750352625386 & 0.1327478726507131\end{array}$

$\begin{array}{llll}0.1994297814565305 & 0.8905735145983883 & 0.1327534423535227\end{array}$

$\begin{array}{lllll}0.0999140001522112 & 0.0546148908781206 & 0.1326749749340279\end{array}$

$\begin{array}{llll}0.0028743350981350 & 0.2223910317504348 & 0.1311000715147944\end{array}$

$\begin{array}{llll}0.4003314271498138 & 0.8907798166856945 & 0.1326704183115377\end{array}$

$\begin{array}{llll}0.6000359750181323 & 0.8910485352084820 & 0.1327572013367951\end{array}$

$\begin{array}{lllll}0.4995288273842651 & 0.0553396828347351 & 0.1320430584038872\end{array}$

$\begin{array}{llll}0.1960490344425717 & 0.2226571676009498 & 0.1309429680581564\end{array}$

$\begin{array}{llll}0.3925547869212440 & 0.2175418252995498 & 0.1352489637508650\end{array}$

$\begin{array}{llll}0.3058136221685686 & 0.3895520786708971 & 0.1357184068364657\end{array}$

$\begin{array}{llll}0.2971263198082013 & 0.0524818791485972 & 0.1315134959612463\end{array}$

$\begin{array}{lllll}0.0029111560664672 & 0.5562875390720050 & 0.1306815675581891\end{array}$

$\begin{array}{llll}0.1964846964914278 & 0.5562858042604133 & 0.1305475717072778\end{array}$

$\begin{array}{llll}0.0999538706825084 & 0.7252600123030688 & 0.1326957033561798\end{array}$

$\begin{array}{llll}0.0993643456532172 & 0.3895394205346670 & 0.1315599636128028\end{array}$

$\begin{array}{llll}0.7996907290382974 & 0.8907413751524251 & 0.1326570830637817\end{array}$

$\begin{array}{llll}0.9027505953300249 & 0.0524712174461713 & 0.1316419026108714\end{array}$

$\begin{array}{llll}0.5997482943546322 & 0.2205624097655512 & 0.1296788610244628\end{array}$

$\begin{array}{llll}0.8064855424955606 & 0.2174665243697071 & 0.1353198693368546\end{array}$

$\begin{array}{llll}0.6959600428879789 & 0.3905328285514323 & 0.1302771411495794\end{array}$

$\begin{array}{llll}0.7005111377227227 & 0.0552622369986884 & 0.1321818949976100\end{array}$

$\begin{array}{llll}0.3940971750080460 & 0.5618421270241987 & 0.1352400125109917\end{array}$

$\begin{array}{llll}0.5998285987800592 & 0.5596559410870655 & 0.1299091455426804\end{array}$

$\begin{array}{llll}0.5000713285704730 & 0.7261851834477729 & 0.1317836870631443\end{array}$

$\begin{array}{llll}0.5036076694947308 & 0.3905383423845419 & 0.1304406462651553\end{array}$

$\begin{array}{llll}0.2972050836143001 & 0.7276768479775529 & 0.1314221323212547\end{array}$

$\begin{array}{llll}0.8056107414576839 & 0.5619202592534663 & 0.1351184307437001\end{array}$

$\begin{array}{llll}0.9027678020380654 & 0.7276091508935703 & 0.1314600434528424\end{array}$

$\begin{array}{llll}0.8934147104421291 & 0.3894682380526369 & 0.1360957241415981\end{array}$

$\begin{array}{llll}0.6999469655038933 & 0.7260058214721773 & 0.1319717033300978\end{array}$

$\begin{array}{lllll}0.2791326035947435 & 0.3885976191573456 & 0.2067447706280019\end{array}$

$\begin{array}{llll}0.5969649100302817 & 0.6943146584564015 & 0.2271663836200881\end{array}$

$\begin{array}{llll}0.9157514845849171 & 0.3892360758744212 & 0.2071928263536662\end{array}$ 
$\begin{array}{llll}0.5976008824275473 & 0.0838729292828860 & 0.2273343681508583\end{array}$

$\begin{array}{lllll}0.9152570725850185 & 0.2029242400877457 & 0.1997603025675694\end{array}$

$\begin{array}{llll}0.7795979889459754 & 0.0759178281080067 & 0.2147511708145272\end{array}$

$\begin{array}{llll}0.4155674613554720 & 0.0758659026616137 & 0.2145193419635749\end{array}$

$\begin{array}{llll}0.2803093408080861 & 0.2028043653492498 & 0.1991591907994422\end{array}$

$\begin{array}{llll}0.2800938254583035 & 0.5747045904998447 & 0.1989672851962920\end{array}$

$\begin{array}{llll}0.4153688551007567 & 0.7018170476455908 & 0.2145675796812506\end{array}$

$\begin{array}{llll}0.7796328837896891 & 0.7021326092729860 & 0.2145657422867040\end{array}$

$\begin{array}{llll}0.9148053078421906 & 0.5745985541748613 & 0.1995414801866310\end{array}$

$\begin{array}{llll}0.6859669379046683 & 0.1991061396913427 & 0.2192764322130289\end{array}$

$\begin{array}{llll}0.7698698440853707 & 0.1492718071848003 & 0.2115279347481057\end{array}$

$\begin{array}{lllll}0.8384519182025871 & 0.2133544066186595 & 0.1986954408752056\end{array}$

$\begin{array}{lllll}0.7931850291969025 & 0.3028027809904556 & 0.2029093772288178\end{array}$

$\begin{array}{llll}0.5975848050017354 & 0.1582707217030470 & 0.2246221946653844\end{array}$

$\begin{array}{llll}0.5092429238505363 & 0.1990693370824483 & 0.2193435926453711\end{array}$

$\begin{array}{lllll}0.4022222278602329 & 0.3026499181115930 & 0.2028049512413213\end{array}$

$\begin{array}{llll}0.3571230009211417 & 0.2133428131418460 & 0.1985618814363253\end{array}$

$\begin{array}{llll}0.4254578803686576 & 0.1491792789172733 & 0.2112895561273621\end{array}$

$\begin{array}{llll}0.3524766924688718 & 0.3888991716165321 & 0.1956244650820225\end{array}$

$\begin{array}{llll}0.4019520446828851 & 0.4749587508079118 & 0.2032106095231866\end{array}$

$\begin{array}{llll}0.5089966629576173 & 0.5786606079840201 & 0.2197819051749612\end{array}$

$\begin{array}{llll}0.4250867432787429 & 0.6283841494534472 & 0.2117465582964169\end{array}$

$\begin{array}{lllll}0.3568682759302237 & 0.5642886924636666 & 0.1989482833673686\end{array}$

$\begin{array}{llll}0.5973232023499618 & 0.6198296754986196 & 0.2247124502182787\end{array}$

$\begin{array}{llll}0.6858507755333179 & 0.5792432453213258 & 0.2195075244592168\end{array}$

$\begin{array}{lllll}0.7927095945942959 & 0.4751169531124384 & 0.2031535052857589\end{array}$

$\begin{array}{llll}0.8425917230138520 & 0.3891113641861909 & 0.1958460591237441\end{array}$

$\begin{array}{llll}0.8379847781441629 & 0.5643261187684433 & 0.1986675221733006\end{array}$

$\begin{array}{llll}0.7696794732598204 & 0.6287876918551344 & 0.2115036340990778\end{array}$

$\begin{array}{llll}0.7016810435925795 & 0.2928295830764893 & 0.2137699560725143\end{array}$

$\begin{array}{llll}0.4935509607014846 & 0.2928014491691422 & 0.2138882680348326\end{array}$

$\begin{array}{llll}0.7014521543704495 & 0.4852743355421303 & 0.2141362786053927\end{array}$

$\begin{array}{llll}0.4932483705414065 & 0.4848567571518355 & 0.2143628720834238\end{array}$

$\begin{array}{llll}0.5974215553301063 & 0.3890054077683689 & 0.1974378889910917\end{array}$

\section{FeP-Pt(111) - B-45, High Spin, vdW-DF-optB88:}

Pt H C N Fe

1.00000000000000

$\begin{array}{lll}14.0790000000000006 & 0.0000000000000000 & 0.0000000000000000\end{array}$

$\begin{array}{lll}0.0000000000000000 & 14.6310000000000002 & 0.0000000000000000\end{array}$

$\begin{array}{llll}0.0000000000000000 & 0.0000000000000000 & 35.0000000000000000\end{array}$

$\mathrm{Pt} \quad \mathrm{H} \quad \mathrm{C} \quad \mathrm{N} \quad \mathrm{Fe}$

$\begin{array}{lllll}90 & 12 & 20 & 4 & 1\end{array}$

Direct

$\begin{array}{llll}0.0000000000000000 & 0.0000000000000000 & 0.0000000000000000\end{array}$

$\begin{array}{lllll}0.2000000000000028 & 0.0000000000000000 & 0.0000000000000000\end{array}$

$\begin{array}{llll}0.3999999999999986 & 0.0000000000000000 & 0.0000000000000000\end{array}$ 
$0.3000100000000003 \quad 0.16667999999999950 .0000000000000000$

$\begin{array}{lllll}0.0000000000000000 & 0.3333399999999997 & 0.0000000000000000\end{array}$

$\begin{array}{llll}0.2000000000000028 & 0.3333399999999997 & 0.0000000000000000\end{array}$

$\begin{array}{llll}0.1000099999999975 & 0.5000199999999992 & 0.0000000000000000\end{array}$

$\begin{array}{llll}0.1000099999999975 & 0.1666799999999995 & 0.0000000000000000\end{array}$

$\begin{array}{llll}0.0000000000000000 & 0.6666999999999987 & 0.0000000000000000\end{array}$

$\begin{array}{llll}0.6000099999999975 & 0.0000000000000000 & 0.0000000000000000\end{array}$

$\begin{array}{lll}0.8000200000000035 & 0.0000000000000000 & 0.0000000000000000\end{array}$

$\begin{array}{llll}0.7000099999999989 & 0.1666799999999995 & 0.0000000000000000\end{array}$

$\begin{array}{llll}0.3999999999999986 & 0.3333399999999997 & 0.0000000000000000\end{array}$

$\begin{array}{lllll}0.6000099999999975 & 0.3333399999999997 & 0.0000000000000000\end{array}$

$\begin{array}{llll}0.5000100000000032 & 0.5000199999999992 & 0.0000000000000000\end{array}$

$\begin{array}{llll}0.5000100000000032 & 0.1666799999999995 & 0.0000000000000000\end{array}$

$\begin{array}{llll}0.2000000000000028 & 0.6666999999999987 & 0.0000000000000000\end{array}$

$\begin{array}{llll}0.3999999999999986 & 0.6666999999999987 & 0.0000000000000000\end{array}$

$\begin{array}{lllll}0.3000100000000003 & 0.8333599999999990 & 0.0000000000000000\end{array}$

$\begin{array}{llll}0.3000100000000003 & 0.5000199999999992 & 0.0000000000000000\end{array}$

$\begin{array}{lllll}0.1000099999999975 & 0.8333599999999990 & 0.0000000000000000\end{array}$

$\begin{array}{llll}0.8000200000000035 & 0.3333399999999997 & 0.0000000000000000\end{array}$

$\begin{array}{llll}0.9000199999999978 & 0.5000199999999992 & 0.0000000000000000\end{array}$

$\begin{array}{llll}0.9000199999999978 & 0.1666799999999995 & 0.0000000000000000\end{array}$

$\begin{array}{lllll}0.6000099999999975 & 0.6666999999999987 & 0.0000000000000000\end{array}$

$\begin{array}{llll}0.8000200000000035 & 0.6666999999999987 & 0.0000000000000000\end{array}$

$\begin{array}{llll}0.7000099999999989 & 0.8333599999999990 & 0.0000000000000000\end{array}$

$\begin{array}{llll}0.7000099999999989 & 0.5000199999999992 & 0.0000000000000000\end{array}$

$\begin{array}{lllll}0.5000100000000032 & 0.8333599999999990 & 0.0000000000000000\end{array}$

$\begin{array}{lllll}0.9000199999999978 & 0.8333599999999990 & 0.0000000000000000\end{array}$

$\begin{array}{llll}0.3000100000000003 & 0.9444400000000002 & 0.0656899999999965\end{array}$

$\begin{array}{lllll}0.0000000000000000 & 0.1111199999999997 & 0.0656899999999965\end{array}$

$\begin{array}{llll}0.2000000000000028 & 0.1111199999999997 & 0.0656899999999965\end{array}$

$\begin{array}{lllll}0.1000099999999975 & 0.2777900000000031 & 0.0656899999999965\end{array}$

$\begin{array}{llll}0.1000099999999975 & 0.9444400000000002 & 0.0656899999999965\end{array}$

$\begin{array}{llll}0.0000000000000000 & 0.4444599999999994 & 0.0656899999999965\end{array}$

$\begin{array}{llll}0.7000099999999989 & 0.9444400000000002 & 0.0656899999999965\end{array}$

$\begin{array}{llll}0.3999999999999986 & 0.1111199999999997 & 0.0656899999999965\end{array}$

$\begin{array}{lllll}0.6000200000000007 & 0.1111199999999997 & 0.0656899999999965\end{array}$

$\begin{array}{lllll}0.5000100000000032 & 0.2777900000000031 & 0.0656899999999965\end{array}$

$\begin{array}{llll}0.5000100000000032 & 0.9444400000000002 & 0.0656899999999965\end{array}$

$\begin{array}{llll}0.2000000000000028 & 0.4444599999999994 & 0.0656899999999965\end{array}$

$\begin{array}{llll}0.3999999999999986 & 0.4444599999999994 & 0.0656899999999965\end{array}$

$\begin{array}{llll}0.3000100000000003 & 0.6111399999999989 & 0.0656899999999965\end{array}$

$\begin{array}{llll}0.3000100000000003 & 0.2777900000000031 & 0.0656899999999965\end{array}$

$\begin{array}{llll}0.0000000000000000 & 0.7778100000000023 & 0.0656899999999965\end{array}$

$\begin{array}{llll}0.2000000000000028 & 0.7778100000000023 & 0.0656899999999965\end{array}$

$\begin{array}{lllll}0.1000099999999975 & 0.6111399999999989 & 0.0656899999999965\end{array}$

$\begin{array}{lllll}0.8000200000000035 & 0.1111199999999997 & 0.0656899999999965\end{array}$

$\begin{array}{lllll}0.9000199999999978 & 0.2777900000000031 & 0.0656899999999965\end{array}$ 
$\begin{array}{llll}0.9000199999999978 & 0.9444400000000002 & 0.0656899999999965\end{array}$ $\begin{array}{llll}0.6000200000000007 & 0.4444599999999994 & 0.0656899999999965\end{array}$ $\begin{array}{lllll}0.8000200000000035 & 0.4444599999999994 & 0.0656899999999965\end{array}$ $\begin{array}{llll}0.7000099999999989 & 0.6111399999999989 & 0.0656899999999965\end{array}$ $\begin{array}{lllll}0.7000099999999989 & 0.2777900000000031 & 0.0656899999999965\end{array}$ $\begin{array}{llll}0.3999999999999986 & 0.7778100000000023 & 0.0656899999999965\end{array}$ $\begin{array}{llll}0.6000200000000007 & 0.7778100000000023 & 0.0656899999999965\end{array}$ $\begin{array}{llll}0.5000100000000032 & 0.6111399999999989 & 0.0656899999999965\end{array}$ $\begin{array}{llll}0.8000200000000035 & 0.7778100000000023 & 0.0656899999999965\end{array}$ $\begin{array}{llll}0.9000199999999978 & 0.6111399999999989 & 0.0656899999999965\end{array}$ $\begin{array}{llll}0.0004736103799145 & 0.8905337478016403 & 0.1326081694951038\end{array}$ $\begin{array}{llll}0.1987754538448399 & 0.8904346682482027 & 0.1326240246557179\end{array}$ $\begin{array}{llll}0.0995837421743749 & 0.0546674749227190 & 0.1328057345597986\end{array}$ $\begin{array}{llll}0.0018748091017916 & 0.2181918491756236 & 0.1316038445859979\end{array}$ $\begin{array}{llll}0.3984965455445509 & 0.8911066111708149 & 0.1322447927833718\end{array}$ $\begin{array}{llll}0.5997152760527819 & 0.8911244098762864 & 0.1309726371256948\end{array}$ $\begin{array}{llll}0.5009705696043002 & 0.0561759254081177 & 0.1355066675401095\end{array}$ $\begin{array}{llll}0.1974639788286575 & 0.2185192161657312 & 0.1311726554079440\end{array}$ $\begin{array}{llll}0.3980167430016346 & 0.2188509022480484 & 0.1335828664441218\end{array}$ $\begin{array}{llll}0.2932372680707459 & 0.3852797164871439 & 0.1361934625588808\end{array}$ $\begin{array}{llll}0.2976498729290746 & 0.0538463356210584 & 0.1321132474088295\end{array}$ $\begin{array}{llll}0.0006212405877058 & 0.5594673624776886 & 0.1315244190305144\end{array}$ $\begin{array}{llll}0.1992982040342959 & 0.5597521403548510 & 0.1314830403522663\end{array}$ $\begin{array}{llll}0.0999358139600676 & 0.7252820356995026 & 0.1324047148418449\end{array}$ $\begin{array}{llll}0.0997589244647525 & 0.3911037491705048 & 0.1307947088094386\end{array}$ $\begin{array}{llll}0.8008644841975943 & 0.8909284071293280 & 0.1321983129538066\end{array}$ $\begin{array}{llll}0.9015229070960064 & 0.0541045486270460 & 0.1321923204833302\end{array}$ $\begin{array}{llll}0.5997899084358183 & 0.2311068089428062 & 0.1284901565790371\end{array}$ $\begin{array}{llll}0.8011889245997503 & 0.2187489566865925 & 0.1337600132207464\end{array}$ $\begin{array}{llll}0.7045373616710328 & 0.3930730296315885 & 0.1297862481740019\end{array}$ $\begin{array}{llll}0.6979856432210062 & 0.0560951960472243 & 0.1356618487772581\end{array}$ $\begin{array}{llll}0.3991407759680122 & 0.5611848307196581 & 0.1339881914392038\end{array}$ $\begin{array}{llll}0.6019007465937585 & 0.5548878499324991 & 0.1279707501092125\end{array}$ $\begin{array}{llll}0.5005032901540793 & 0.7263755884446128 & 0.1356028608853203\end{array}$ $\begin{array}{llll}0.4954842085139730 & 0.3934440114317580 & 0.1296797834686947\end{array}$ $\begin{array}{llll}0.2986968456208141 & 0.7265169249065468 & 0.1316031247929663\end{array}$ $\begin{array}{llll}0.8019757737310991 & 0.5613696933154561 & 0.1343351267604307\end{array}$ $\begin{array}{llll}0.9012812790482088 & 0.7265278311646765 & 0.1316066966548127\end{array}$ $\begin{array}{llll}0.9063606022678183 & 0.3841234806308504 & 0.1361574302371713\end{array}$ $\begin{array}{llll}0.6983212490114963 & 0.7265277007786065 & 0.1358704817141568\end{array}$ $\begin{array}{llll}0.3684205457526820 & 0.6134205180610814 & 0.2050770628289767\end{array}$ $\begin{array}{llll}0.8265791975462747 & 0.6141192576638125 & 0.2055387171676588\end{array}$ $\begin{array}{llll}0.8280100327556390 & 0.1731955446304698 & 0.2049197245483221\end{array}$ $\begin{array}{llll}0.3683705005908479 & 0.1723161549405547 & 0.2046212698491051\end{array}$ $\begin{array}{llll}0.6898835976991714 & 0.0401436309600139 & 0.2102722979653888\end{array}$ $\begin{array}{llll}0.5072027629234058 & 0.0397989086511651 & 0.2102118573829247\end{array}$ $\begin{array}{llll}0.2350568912313885 & 0.3008389371754453 & 0.2050550783550905\end{array}$ 
$\begin{array}{lll}0.2360477341832272 & 0.4877858641345242 & 0.2074362423008169\end{array}$

$\begin{array}{lllll}0.5066403632180789 & 0.7462802248348527 & 0.2099274353497527\end{array}$

$\begin{array}{lllll}0.6889557381738087 & 0.7459986475264347 & 0.2099139259247835\end{array}$

$\begin{array}{llll}0.9590532494793972 & 0.4892087423029602 & 0.2086259475538784\end{array}$

$\begin{array}{llll}0.9609299724589487 & 0.3021961484296113 & 0.2056177717756516\end{array}$

$\begin{array}{llll}0.5181995783369926 & 0.1882060477179577 & 0.1978949208927498\end{array}$

$\begin{array}{llll}0.5450273726448291 & 0.0911496669500251 & 0.1932162307584990\end{array}$

$\begin{array}{lllll}0.6519601307477918 & 0.0914088188279774 & 0.1932362572054108\end{array}$

$\begin{array}{llll}0.6781912993703941 & 0.1885186861020239 & 0.1977822148212793\end{array}$

$\begin{array}{llll}0.4226810575076001 & 0.2231944991734878 & 0.1973809567224463\end{array}$

$\begin{array}{llll}0.3942170233945814 & 0.3168851006006065 & 0.2039543430376068\end{array}$

$\begin{array}{llll}0.3938830263240705 & 0.4692048529604658 & 0.2070279634094435\end{array}$

$\begin{array}{lllll}0.2970015097096430 & 0.4425435201648646 & 0.2047177599028930\end{array}$

$\begin{array}{llll}0.2957694101889952 & 0.3459042259200339 & 0.2008138170221245\end{array}$

$\begin{array}{llll}0.4231057943168562 & 0.5618853913233606 & 0.1993384655133116\end{array}$

$\begin{array}{llll}0.5181130975438853 & 0.5968518355558428 & 0.1996642824323751\end{array}$

$\begin{array}{llll}0.6774166571122103 & 0.5972331450122976 & 0.1989331702706366\end{array}$

$\begin{array}{llll}0.6510484899801814 & 0.6939092785341447 & 0.1932753011103802\end{array}$

$\begin{array}{llll}0.5442216566687103 & 0.6937488967257546 & 0.1934572329659672\end{array}$

$\begin{array}{llll}0.7725732100078749 & 0.5624325804219106 & 0.1990121654283264\end{array}$

$\begin{array}{llll}0.8016572565932945 & 0.4698656744450236 & 0.2074116813185341\end{array}$

$\begin{array}{lllll}0.8018242511444084 & 0.3178136961310847 & 0.2034318890757979\end{array}$

$\begin{array}{llll}0.7737624707246759 & 0.2237839873342204 & 0.1972381287746290\end{array}$

$\begin{array}{llll}0.9003723919819580 & 0.3472896016267922 & 0.2008701219307849\end{array}$

$\begin{array}{llll}0.8983184338298016 & 0.4436389254500260 & 0.2060045295291053\end{array}$

$\begin{array}{lllll}0.5980636995587989 & 0.2432860076159609 & 0.1991579299758169\end{array}$

$\begin{array}{llll}0.4516374802185322 & 0.3919710924664849 & 0.2069768667415870\end{array}$

$\begin{array}{llll}0.7440446372656399 & 0.3924269749846769 & 0.2063842982175864\end{array}$

$\begin{array}{llll}0.5977103626065566 & 0.5424408262990393 & 0.2015047117013408\end{array}$

$\begin{array}{llll}0.5976559940337081 & 0.3938716922658074 & 0.1929718971676730\end{array}$

\section{FeP-Pt(111) - B-45, Intermediate Spin, vdW-DF-optB88:}

Pt H C N Fe

1.00000000000000

$\begin{array}{lll}14.0790000000000006 & 0.0000000000000000 & 0.0000000000000000\end{array}$

$\begin{array}{llll}0.0000000000000000 & 14.6310000000000002 & 0.0000000000000000\end{array}$

$\begin{array}{llll}0.0000000000000000 & 0.0000000000000000 & 35.0000000000000000\end{array}$

$\mathrm{Pt} \quad \mathrm{H} \quad \mathrm{C} \quad \mathrm{N} \quad \mathrm{Fe}$

$\begin{array}{lllll}90 & 12 & 20 & 4 & 1\end{array}$

Direct

$\begin{array}{llll}0.0000000000000000 & 0.0000000000000000 & 0.0000000000000000\end{array}$

$\begin{array}{lll}0.2000000000000028 & 0.0000000000000000 & 0.0000000000000000\end{array}$

$\begin{array}{llll}0.3999999999999986 & 0.0000000000000000 & 0.0000000000000000\end{array}$

$\begin{array}{lll}0.3000100000000003 & 0.1666799999999995 & 0.0000000000000000\end{array}$

$\begin{array}{lllll}0.0000000000000000 & 0.3333399999999997 & 0.0000000000000000\end{array}$

$\begin{array}{lllll}0.2000000000000028 & 0.3333399999999997 & 0.0000000000000000\end{array}$

$\begin{array}{llll}0.1000099999999975 & 0.5000199999999992 & 0.0000000000000000\end{array}$ 
$\begin{array}{llll}0.1000099999999975 & 0.1666799999999995 & 0.0000000000000000\end{array}$

$\begin{array}{lllll}0.0000000000000000 & 0.6666999999999987 & 0.0000000000000000\end{array}$

$\begin{array}{lllll}0.6000099999999975 & 0.0000000000000000 & 0.0000000000000000\end{array}$

$\begin{array}{lllll}0.8000200000000035 & 0.0000000000000000 & 0.0000000000000000\end{array}$

$\begin{array}{llll}0.7000099999999989 & 0.1666799999999995 & 0.0000000000000000\end{array}$

$\begin{array}{lllll}0.3999999999999986 & 0.3333399999999997 & 0.0000000000000000\end{array}$

$\begin{array}{lllll}0.6000099999999975 & 0.3333399999999997 & 0.0000000000000000\end{array}$

$\begin{array}{llll}0.5000100000000032 & 0.5000199999999992 & 0.0000000000000000\end{array}$

$\begin{array}{llll}0.5000100000000032 & 0.1666799999999995 & 0.0000000000000000\end{array}$

$\begin{array}{llll}0.2000000000000028 & 0.6666999999999987 & 0.0000000000000000\end{array}$

$\begin{array}{lllll}0.3999999999999986 & 0.6666999999999987 & 0.0000000000000000\end{array}$

$\begin{array}{lllll}0.3000100000000003 & 0.8333599999999990 & 0.0000000000000000\end{array}$

$\begin{array}{llll}0.3000100000000003 & 0.5000199999999992 & 0.0000000000000000\end{array}$

$\begin{array}{llll}0.1000099999999975 & 0.8333599999999990 & 0.0000000000000000\end{array}$

$\begin{array}{llll}0.8000200000000035 & 0.3333399999999997 & 0.0000000000000000\end{array}$

$\begin{array}{lllll}0.9000199999999978 & 0.5000199999999992 & 0.0000000000000000\end{array}$

$\begin{array}{llll}0.9000199999999978 & 0.1666799999999995 & 0.0000000000000000\end{array}$

$\begin{array}{lllll}0.6000099999999975 & 0.6666999999999987 & 0.0000000000000000\end{array}$

$\begin{array}{llll}0.8000200000000035 & 0.6666999999999987 & 0.0000000000000000\end{array}$

$\begin{array}{llll}0.7000099999999989 & 0.8333599999999990 & 0.0000000000000000\end{array}$

$\begin{array}{llll}0.7000099999999989 & 0.5000199999999992 & 0.0000000000000000\end{array}$

$\begin{array}{lllll}0.5000100000000032 & 0.8333599999999990 & 0.0000000000000000\end{array}$

$\begin{array}{lllll}0.9000199999999978 & 0.8333599999999990 & 0.0000000000000000\end{array}$

$\begin{array}{llll}0.3000100000000003 & 0.9444400000000002 & 0.0656899999999965\end{array}$

$\begin{array}{lllll}0.0000000000000000 & 0.1111199999999997 & 0.0656899999999965\end{array}$

$\begin{array}{lllll}0.2000000000000028 & 0.1111199999999997 & 0.0656899999999965\end{array}$

$\begin{array}{lllll}0.1000099999999975 & 0.2777900000000031 & 0.0656899999999965\end{array}$

$\begin{array}{llll}0.1000099999999975 & 0.9444400000000002 & 0.0656899999999965\end{array}$

$\begin{array}{llll}0.0000000000000000 & 0.4444599999999994 & 0.0656899999999965\end{array}$

$\begin{array}{llll}0.7000099999999989 & 0.9444400000000002 & 0.0656899999999965\end{array}$

$\begin{array}{lllll}0.3999999999999986 & 0.1111199999999997 & 0.0656899999999965\end{array}$

$\begin{array}{llllll}0.6000200000000007 & 0.1111199999999997 & 0.0656899999999965\end{array}$

$\begin{array}{llll}0.5000100000000032 & 0.2777900000000031 & 0.0656899999999965\end{array}$

$\begin{array}{llll}0.5000100000000032 & 0.9444400000000002 & 0.0656899999999965\end{array}$

$\begin{array}{llll}0.2000000000000028 & 0.4444599999999994 & 0.0656899999999965\end{array}$

$\begin{array}{lllll}0.3999999999999986 & 0.4444599999999994 & 0.0656899999999965\end{array}$

$\begin{array}{llll}0.3000100000000003 & 0.6111399999999989 & 0.0656899999999965\end{array}$

$\begin{array}{llll}0.3000100000000003 & 0.2777900000000031 & 0.0656899999999965\end{array}$

$\begin{array}{llll}0.0000000000000000 & 0.7778100000000023 & 0.0656899999999965\end{array}$

$\begin{array}{llll}0.2000000000000028 & 0.7778100000000023 & 0.0656899999999965\end{array}$

$\begin{array}{lllll}0.1000099999999975 & 0.6111399999999989 & 0.0656899999999965\end{array}$

$\begin{array}{llll}0.8000200000000035 & 0.1111199999999997 & 0.0656899999999965\end{array}$

$\begin{array}{lllll}0.9000199999999978 & 0.2777900000000031 & 0.0656899999999965\end{array}$

$\begin{array}{llll}0.9000199999999978 & 0.9444400000000002 & 0.0656899999999965\end{array}$

$\begin{array}{lllll}0.6000200000000007 & 0.4444599999999994 & 0.0656899999999965\end{array}$

$\begin{array}{lllll}0.8000200000000035 & 0.4444599999999994 & 0.0656899999999965\end{array}$

$\begin{array}{llll}0.7000099999999989 & 0.6111399999999989 & 0.0656899999999965\end{array}$ 
$\begin{array}{llll}0.7000099999999989 & 0.2777900000000031 & 0.0656899999999965\end{array}$ $\begin{array}{llll}0.3999999999999986 & 0.7778100000000023 & 0.0656899999999965\end{array}$ $\begin{array}{llll}0.6000200000000007 & 0.7778100000000023 & 0.0656899999999965\end{array}$ $\begin{array}{llll}0.5000100000000032 & 0.6111399999999989 & 0.0656899999999965\end{array}$ $\begin{array}{llll}0.8000200000000035 & 0.7778100000000023 & 0.0656899999999965\end{array}$ $\begin{array}{llll}0.9000199999999978 & 0.6111399999999989 & 0.0656899999999965\end{array}$ $\begin{array}{llll}0.0005345578225402 & 0.8906402658511468 & 0.1326354528899714\end{array}$ $\begin{array}{llll}0.1989102662209845 & 0.8905445728834422 & 0.1326583177299696\end{array}$ $\begin{array}{lllll}0.0996120130852987 & 0.0546372673680864 & 0.1328907120919425\end{array}$ $\begin{array}{llll}0.0019774700552517 & 0.2182760584583008 & 0.1315414181734056\end{array}$ $\begin{array}{llll}0.3986335693264351 & 0.8909403223439369 & 0.1321471093303472\end{array}$ $\begin{array}{lllll}0.5995917237028578 & 0.8910088584545974 & 0.1311499614045033\end{array}$ $\begin{array}{llll}0.5004935087594049 & 0.0565344813390780 & 0.1356352157617238\end{array}$ $\begin{array}{llll}0.1974147542952025 & 0.2184667465349790 & 0.1311770099283009\end{array}$ $\begin{array}{llll}0.3980052452420253 & 0.2189024834470032 & 0.1335780424226201\end{array}$ $\begin{array}{llll}0.2934661071427342 & 0.3849512063158969 & 0.1359785634476367\end{array}$ $\begin{array}{lllll}0.2976767404314131 & 0.0538752237351190 & 0.1321196565713194\end{array}$ $\begin{array}{llll}0.0005890782896003 & 0.5594108916963592 & 0.1314828288400705\end{array}$ $\begin{array}{llll}0.1993322444082963 & 0.5596832485371124 & 0.1314573814772402\end{array}$ $\begin{array}{llll}0.1000035467828368 & 0.7254623061092502 & 0.1324129049986595\end{array}$ $\begin{array}{llll}0.0997903149731272 & 0.3910531602887417 & 0.1308527679518363\end{array}$ $\begin{array}{llll}0.8007271585884581 & 0.8909342517696714 & 0.1321777356581180\end{array}$ $\begin{array}{llll}0.9014658485395515 & 0.0540758321990964 & 0.1321948380108466\end{array}$ $\begin{array}{llll}0.5998015093705007 & 0.2306007112369528 & 0.1283109722084887\end{array}$ $\begin{array}{lllll}0.8011676976304116 & 0.2187536300893314 & 0.1337286402044668\end{array}$ $\begin{array}{llll}0.7039820932852905 & 0.3926445484635579 & 0.1296688750966837\end{array}$ $\begin{array}{llll}0.6976479842211631 & 0.0562870189504423 & 0.1357965195709809\end{array}$ $\begin{array}{llll}0.3990177553482610 & 0.5610408034462253 & 0.1337601307128224\end{array}$ $\begin{array}{llll}0.6019730512065209 & 0.5549133071985040 & 0.1279190273424504\end{array}$ $\begin{array}{llll}0.5007723489809095 & 0.7259385371506610 & 0.1357388293396085\end{array}$ $\begin{array}{llll}0.4963563250943182 & 0.3926357058463950 & 0.1296403767716825\end{array}$ $\begin{array}{llll}0.2987064667829316 & 0.7265426715078860 & 0.1316045690066332\end{array}$ $\begin{array}{llll}0.8020431599742537 & 0.5613648842841883 & 0.1340881150389421\end{array}$ $\begin{array}{llll}0.9013014374580237 & 0.7265572472315540 & 0.1316049891131925\end{array}$ $\begin{array}{llll}0.9062525518170617 & 0.3840447722202072 & 0.1359741839947759\end{array}$ $\begin{array}{llll}0.6984331629242959 & 0.7263245006768102 & 0.1359751002585057\end{array}$ $\begin{array}{llll}0.3690831446087302 & 0.6136802386628801 & 0.2050063092145962\end{array}$ $\begin{array}{llll}0.8253710206626295 & 0.6140504012155930 & 0.2055052107626736\end{array}$ $\begin{array}{llll}0.8271316488284910 & 0.1736595514022170 & 0.2049548261851371\end{array}$ $\begin{array}{lllll}0.3690147383743110 & 0.1730506793646711 & 0.2047766845092482\end{array}$ $\begin{array}{llll}0.6895873337666814 & 0.0429071725233570 & 0.2101949891094890\end{array}$ $\begin{array}{llll}0.5070950304392667 & 0.0426314472527736 & 0.2102689023091244\end{array}$ $\begin{array}{llll}0.2368999021847600 & 0.3008319211304382 & 0.2050934128008635\end{array}$ $\begin{array}{llll}0.2373678421535814 & 0.4878987382449012 & 0.2077316910442235\end{array}$ $\begin{array}{llll}0.5070547469765430 & 0.7435155575793075 & 0.2100115611494928\end{array}$ $\begin{array}{llll}0.6896056617055919 & 0.7422923790961633 & 0.2101058279269594\end{array}$ $\begin{array}{llll}0.9574864584366765 & 0.4889867425777226 & 0.2088201378267911\end{array}$ 
$\begin{array}{llll}0.9587857959030472 & 0.3019276738297805 & 0.2056130332008408\end{array}$

$\begin{array}{llll}0.5187303809649322 & 0.1909916257919875 & 0.1980566337923761\end{array}$

$\begin{array}{llll}0.5449441117815468 & 0.0938326714038027 & 0.1931418496686064\end{array}$

$\begin{array}{llll}0.6513652770064269 & 0.0938695231611177 & 0.1930518075475618\end{array}$

$\begin{array}{llll}0.6775622307631721 & 0.1911340101714885 & 0.1974766759162421\end{array}$

$\begin{array}{llll}0.4231301398150791 & 0.2241482505307957 & 0.1976045068424739\end{array}$

$\begin{array}{llll}0.3956203004751941 & 0.3174485029200369 & 0.2036832791172358\end{array}$

$\begin{array}{llll}0.3948594112525043 & 0.4695386813834261 & 0.2068875960571859\end{array}$

$\begin{array}{llll}0.2984012726986123 & 0.4426664089036976 & 0.2052611900525463\end{array}$

$\begin{array}{llll}0.2973519047902542 & 0.3463152752217908 & 0.2009799515831716\end{array}$

$\begin{array}{llll}0.4231439057454267 & 0.5617259804107704 & 0.1990102013263808\end{array}$

$\begin{array}{llll}0.5184231344698915 & 0.5945190397400282 & 0.1992106052448079\end{array}$

$\begin{array}{llll}0.6766798028673122 & 0.5940867273566113 & 0.1983587446847963\end{array}$

$\begin{array}{llll}0.6512082921761433 & 0.6911189929116475 & 0.1931439143935165\end{array}$

$\begin{array}{llll}0.5447289938816908 & 0.6913826320205985 & 0.1933192557240133\end{array}$

$\begin{array}{llll}0.7721754614297183 & 0.5617790659715993 & 0.1987881608374096\end{array}$

$\begin{array}{llll}0.8003951762914099 & 0.4697671021787642 & 0.2071239638761782\end{array}$

$\begin{array}{llll}0.8002258335692289 & 0.3180938820443870 & 0.2029563972324340\end{array}$

$\begin{array}{llll}0.7732586361263500 & 0.2244272387130692 & 0.1971053872969719\end{array}$

$\begin{array}{llll}0.8984880615752218 & 0.3474341523079048 & 0.2010189339347548\end{array}$

$\begin{array}{llll}0.8966683489530354 & 0.4434487461935603 & 0.2063430138955837\end{array}$

$\begin{array}{llll}0.5980363370528750 & 0.2473187965374208 & 0.1992896530722135\end{array}$

$\begin{array}{llll}0.4540329002946137 & 0.3926139731328107 & 0.2062397611734497\end{array}$

$\begin{array}{llll}0.7413166296915179 & 0.3927609640489251 & 0.2053556195748385\end{array}$

$\begin{array}{llll}0.5972858609263128 & 0.5382182920100931 & 0.2009020171094136\end{array}$

$\begin{array}{llll}0.5974601213879254 & 0.3931597938192084 & 0.1999105592514354\end{array}$

\section{FeP-Pt(111) - T-0, vdW-DF-revPBE:}

Pt Fe N C H

1.00000000000000

$\begin{array}{llll}14.2799999999999994 & 0.0000000000000000 & 0.0000000000000000\end{array}$

$\begin{array}{llll}0.0000000000000000 & 14.8409999999999993 & 0.0000000000000000\end{array}$

$\begin{array}{llll}0.0000000000000000 & 0.0000000000000000 & 35.0000000000000000\end{array}$

$\mathrm{Pt} F \mathrm{~N}$ C $\mathrm{H}$

$\begin{array}{lllll}90 & 1 & 4 & 20 & 12\end{array}$

Direct

$\begin{array}{llll}0.7999999999999972 & 0.6666669999999968 & 0.0000000000000000\end{array}$

$\begin{array}{llll}0.9000040000000027 & 0.8333319999999986 & 0.0000000000000000\end{array}$

$\begin{array}{llll}0.7999999999999972 & 0.3333330000000032 & 0.0000000000000000\end{array}$

$\begin{array}{llll}0.9000040000000027 & 0.4999990000000025 & 0.0000000000000000\end{array}$

$\begin{array}{lllll}0.7999999999999972 & 0.0000000000000000 & 0.0000000000000000\end{array}$

$\begin{array}{llll}0.9000040000000027 & 0.1666650000000018 & 0.0000000000000000\end{array}$

$\begin{array}{llll}0.6000090000000000 & 0.6666669999999968 & 0.0000000000000000\end{array}$

$\begin{array}{llll}0.7000140000000030 & 0.8333319999999986 & 0.0000000000000000\end{array}$

$\begin{array}{lllll}0.3999999999999986 & 0.6666669999999968 & 0.0000000000000000\end{array}$

$\begin{array}{llll}0.5000039999999970 & 0.8333319999999986 & 0.0000000000000000\end{array}$

$\begin{array}{llll}0.6000090000000000 & 0.3333330000000032 & 0.0000000000000000\end{array}$ 
$\begin{array}{lll}0.7000140000000030 & 0.4999990000000025 & 0.0000000000000000\end{array}$

$\begin{array}{llll}0.3999999999999986 & 0.3333330000000032 & 0.0000000000000000\end{array}$

$\begin{array}{llll}0.5000039999999970 & 0.4999990000000025 & 0.0000000000000000\end{array}$

$\begin{array}{llll}0.6000090000000000 & 0.0000000000000000 & 0.0000000000000000\end{array}$

$\begin{array}{llll}0.7000140000000030 & 0.1666650000000018 & 0.0000000000000000\end{array}$

$\begin{array}{llll}0.3999999999999986 & 0.0000000000000000 & 0.0000000000000000\end{array}$

$\begin{array}{llll}0.5000039999999970 & 0.1666650000000018 & 0.0000000000000000\end{array}$

$\begin{array}{llll}0.2000090000000014 & 0.6666669999999968 & 0.0000000000000000\end{array}$

$\begin{array}{llll}0.3000139999999973 & 0.8333319999999986 & 0.0000000000000000\end{array}$

$\begin{array}{llll}0.0000000000000000 & 0.6666669999999968 & 0.0000000000000000\end{array}$

$\begin{array}{lll}0.1000039999999984 & 0.8333319999999986 & 0.0000000000000000\end{array}$

$\begin{array}{llll}0.2000090000000014 & 0.3333330000000032 & 0.0000000000000000\end{array}$

$\begin{array}{llll}0.3000139999999973 & 0.4999990000000025 & 0.0000000000000000\end{array}$

$\begin{array}{llll}0.0000000000000000 & 0.3333330000000032 & 0.0000000000000000\end{array}$

$\begin{array}{llll}0.1000039999999984 & 0.4999990000000025 & 0.0000000000000000\end{array}$

$\begin{array}{lllll}0.2000090000000014 & 0.0000000000000000 & 0.0000000000000000\end{array}$

$\begin{array}{llll}0.3000139999999973 & 0.1666650000000018 & 0.0000000000000000\end{array}$

$\begin{array}{llll}0.0000000000000000 & 0.0000000000000000 & 0.0000000000000000\end{array}$

0.10000399999999840 .16666500000000180 .0000000000000000

$\begin{array}{llll}0.9000040000000027 & 0.7222220000000021 & 0.0666289999999989\end{array}$

$\begin{array}{llll}0.7999999999999972 & 0.8888869999999969 & 0.0666289999999989\end{array}$

$\begin{array}{lllll}0.9000040000000027 & 0.3888880000000015 & 0.0666289999999989\end{array}$

$\begin{array}{llll}0.7999999999999972 & 0.5555540000000008 & 0.0666289999999989\end{array}$

$\begin{array}{llll}0.9000040000000027 & 0.0555549999999982 & 0.0666289999999989\end{array}$

$\begin{array}{lllll}0.7999999999999972 & 0.2222209999999976 & 0.0666289999999989\end{array}$

$\begin{array}{lllll}0.6000090000000000 & 0.8888869999999969 & 0.0666289999999989\end{array}$

$\begin{array}{llll}0.7000140000000030 & 0.7222220000000021 & 0.0666289999999989\end{array}$

$\begin{array}{lllll}0.5000039999999970 & 0.7222220000000021 & 0.0666289999999989\end{array}$

$\begin{array}{llll}0.3999999999999986 & 0.8888869999999969 & 0.0666289999999989\end{array}$

$\begin{array}{llll}0.6000090000000000 & 0.5555540000000008 & 0.0666289999999989\end{array}$

$\begin{array}{llll}0.7000140000000030 & 0.3888880000000015 & 0.0666289999999989\end{array}$

$\begin{array}{llll}0.5000039999999970 & 0.3888880000000015 & 0.0666289999999989\end{array}$

$\begin{array}{llll}0.3999999999999986 & 0.5555540000000008 & 0.0666289999999989\end{array}$

$\begin{array}{llll}0.6000090000000000 & 0.2222209999999976 & 0.0666289999999989\end{array}$

$\begin{array}{llll}0.7000140000000030 & 0.0555549999999982 & 0.0666289999999989\end{array}$

$\begin{array}{lllll}0.5000039999999970 & 0.0555549999999982 & 0.0666289999999989\end{array}$

$\begin{array}{lllll}0.3999999999999986 & 0.2222209999999976 & 0.0666289999999989\end{array}$

$\begin{array}{lllll}0.2000090000000014 & 0.8888869999999969 & 0.0666289999999989\end{array}$

$\begin{array}{llll}0.3000139999999973 & 0.7222220000000021 & 0.0666289999999989\end{array}$

$\begin{array}{lllll}0.1000039999999984 & 0.7222220000000021 & 0.0666289999999989\end{array}$

$\begin{array}{llll}0.0000000000000000 & 0.8888869999999969 & 0.0666289999999989\end{array}$

$\begin{array}{llll}0.2000090000000014 & 0.5555540000000008 & 0.0666289999999989\end{array}$

$\begin{array}{llll}0.3000139999999973 & 0.3888880000000015 & 0.0666289999999989\end{array}$

$\begin{array}{llll}0.1000039999999984 & 0.3888880000000015 & 0.0666289999999989\end{array}$

$\begin{array}{lllll}0.0000000000000000 & 0.5555540000000008 & 0.0666289999999989\end{array}$

$\begin{array}{lllll}0.2000090000000014 & 0.2222209999999976 & 0.0666289999999989\end{array}$

$\begin{array}{llll}0.3000139999999973 & 0.0555549999999982 & 0.0666289999999989\end{array}$ 
$\begin{array}{llll}0.1000039999999984 & 0.0555549999999982 & 0.0666289999999989\end{array}$ $\begin{array}{lllll}0.0000000000000000 & 0.2222209999999976 & 0.0666289999999989\end{array}$ $\begin{array}{llll}0.7993594450792009 & 0.7809226334248732 & 0.1342933540976212\end{array}$ $\begin{array}{llll}0.8974395021629147 & 0.9458288639215499 & 0.1342472080172572\end{array}$ $\begin{array}{llll}0.8036197664807073 & 0.4469807716077100 & 0.1322491744350222\end{array}$ $\begin{array}{llll}0.8999604537688937 & 0.6144740652500756 & 0.1346507822618783\end{array}$ $\begin{array}{llll}0.7979282032924899 & 0.1112724426243786 & 0.1331591208690593\end{array}$ $\begin{array}{lllll}0.8995108297779392 & 0.2776929252516097 & 0.1337004849453865\end{array}$ $\begin{array}{llll}0.3986384935125164 & 0.7803675536592921 & 0.1345543419102002\end{array}$ $\begin{array}{llll}0.5983786049527924 & 0.7820523232561082 & 0.1340380059697728\end{array}$ $\begin{array}{llll}0.6969548757117873 & 0.9488542290256129 & 0.1340110252707111\end{array}$ $\begin{array}{llll}0.4985780162659816 & 0.9475791216154661 & 0.1343736150890384\end{array}$ $\begin{array}{llll}0.3980948753799726 & 0.4474690679426865 & 0.1352321058824255\end{array}$ $\begin{array}{llll}0.6070681012080952 & 0.4495468099095373 & 0.1374710390576008\end{array}$ $\begin{array}{llll}0.7000642164184698 & 0.6173295332272986 & 0.1333910973846737\end{array}$ $\begin{array}{llll}0.4983968790831584 & 0.6149569014676636 & 0.1321672827292500\end{array}$ $\begin{array}{lllll}0.3955357967248797 & 0.1093859636594721 & 0.1337123713516227\end{array}$ $\begin{array}{llll}0.5921607143197902 & 0.1197132704507086 & 0.1433181728175154\end{array}$ $\begin{array}{llll}0.6988679217688457 & 0.2774530491145888 & 0.1364492033628447\end{array}$ $\begin{array}{llll}0.4939563299400814 & 0.2807662128148970 & 0.1299169407672407\end{array}$ $\begin{array}{llll}0.9980396729035236 & 0.7799526574993618 & 0.1343274817040745\end{array}$ $\begin{array}{llll}0.1983438386373836 & 0.7798990840027759 & 0.1341091130028098\end{array}$ $\begin{array}{llll}0.2977900334034019 & 0.9447340855643639 & 0.1344035245303498\end{array}$ $\begin{array}{llll}0.0978498634235608 & 0.9448591655850158 & 0.1341510557573596\end{array}$ $\begin{array}{llll}0.9978788834087573 & 0.4462376950700847 & 0.1335178528872957\end{array}$ $\begin{array}{llll}0.1936479585977252 & 0.4451224965615872 & 0.1409527196806851\end{array}$ $\begin{array}{llll}0.2983139533484774 & 0.6136831099711288 & 0.1320209309512033\end{array}$ $\begin{array}{llll}0.0988845709718386 & 0.6136172878006237 & 0.1333598429912312\end{array}$ $\begin{array}{llll}0.9982723704856850 & 0.1109990579057948 & 0.1342281302556880\end{array}$ $\begin{array}{llll}0.1969859013670693 & 0.1106031559491001 & 0.1340390004060685\end{array}$ $\begin{array}{llll}0.2949494945873456 & 0.2799681096900981 & 0.1308545595915588\end{array}$ $\begin{array}{llll}0.0972719772528958 & 0.2779785401199914 & 0.1335718314126475\end{array}$ $\begin{array}{llll}0.4101532389901061 & 0.4371098340073310 & 0.2089130540750660\end{array}$ $\begin{array}{llll}0.3087957940234405 & 0.5326818764754562 & 0.2234446369588255\end{array}$ $\begin{array}{llll}0.3136989112681703 & 0.3360063993880028 & 0.2190568282046706\end{array}$ $\begin{array}{llll}0.5159784560816105 & 0.3324300919912627 & 0.2126681400351791\end{array}$ $\begin{array}{llll}0.5105241888986704 & 0.5326092220704073 & 0.2229540386298005\end{array}$ $\begin{array}{llll}0.4146653473705706 & 0.1991483305082821 & 0.2196173129295206\end{array}$ $\begin{array}{llll}0.4093046857796865 & 0.6653352146781374 & 0.2354169639187625\end{array}$ $\begin{array}{llll}0.5014771327273670 & 0.2390506818928020 & 0.2105520598044176\end{array}$ $\begin{array}{llll}0.3216659173865376 & 0.6243404430807398 & 0.2311973368101449\end{array}$ $\begin{array}{llll}0.6069164769558687 & 0.3503941623522400 & 0.2046138695133936\end{array}$ $\begin{array}{llll}0.3281604818858470 & 0.2435332479842030 & 0.2246305299654583\end{array}$ $\begin{array}{llll}0.2185669261292722 & 0.3491734270779842 & 0.2171296066086784\end{array}$ $\begin{array}{llll}0.4972722853333792 & 0.6239333639189480 & 0.2315411030600053\end{array}$ $\begin{array}{llll}0.2149339579078347 & 0.5201082890843638 & 0.2179796618685583\end{array}$ $\begin{array}{llll}0.6045026912805622 & 0.5211268613957498 & 0.2171509040852366\end{array}$ 
$\begin{array}{llll}0.1748050222522045 & 0.4341939799275651 & 0.2071075732720230\end{array}$

$\begin{array}{llll}0.6457834573808763 & 0.4399754710496812 & 0.2019101478276292\end{array}$

$\begin{array}{llll}0.5903104860711434 & 0.1915947067262985 & 0.1999398442779413\end{array}$

$\begin{array}{llll}0.6607607660129272 & 0.2667788492765531 & 0.1960282214365479\end{array}$

$\begin{array}{llll}0.2384289389523436 & 0.2000680328610445 & 0.2290857306330498\end{array}$

$\begin{array}{llll}0.5868376922934572 & 0.6684327265889395 & 0.2326046853889929\end{array}$

$\begin{array}{llll}0.2315562399714239 & 0.6685038000828828 & 0.2316971938763296\end{array}$

$\begin{array}{llll}0.1648959293728635 & 0.6038883481855341 & 0.2232810549027441\end{array}$

$\begin{array}{llll}0.1703499323418302 & 0.2652841852748100 & 0.2238952845500796\end{array}$

$\begin{array}{llll}0.6539454166242648 & 0.6045703237032466 & 0.2235387357566686\end{array}$

$\begin{array}{llll}0.4131585429490498 & 0.1258318674296335 & 0.2207604317967600\end{array}$

$\begin{array}{llll}0.4096523199332668 & 0.7378111192525666 & 0.2403762704418113\end{array}$

$\begin{array}{llll}0.7221875251287219 & 0.4410621427781363 & 0.2014662442869354\end{array}$

$\begin{array}{llll}0.0985999961840349 & 0.4324301534750206 & 0.2088640614930240\end{array}$

$\begin{array}{llll}0.6092703067183413 & 0.1324345089842948 & 0.2169294374833873\end{array}$

$\begin{array}{llll}0.7287534240401791 & 0.2585893970598942 & 0.2099961978831593\end{array}$

$\begin{array}{lllll}0.0896536246167579 & 0.6118994971553458 & 0.2210902072385888\end{array}$

$\begin{array}{llll}0.2206252919627749 & 0.7397749026001061 & 0.2368478362424198\end{array}$

$\begin{array}{llll}0.5976446397142391 & 0.7393606697411540 & 0.2385354877326564\end{array}$

$\begin{array}{llll}0.2280859322369756 & 0.1286466054631768 & 0.2339948417534305\end{array}$

$\begin{array}{llll}0.0949655253271105 & 0.2570017626647703 & 0.2245669510494091\end{array}$

$\begin{array}{llll}0.7291893695033312 & 0.6133666660472959 & 0.2216100661056035\end{array}$

\section{FeP-Pt(111) - T-45, vdW-DF-revPBE:}

$\mathrm{Pt} \mathrm{Fe} \mathrm{N} \mathrm{C} \mathrm{H}$

1.00000000000000

$\begin{array}{llll}14.2799999999999994 & 0.0000000000000000 & 0.0000000000000000\end{array}$

$\begin{array}{llll}0.0000000000000000 & 14.8409999999999993 & 0.0000000000000000\end{array}$

$\begin{array}{llll}0.0000000000000000 & 0.0000000000000000 & 35.0000000000000000\end{array}$

$\mathrm{Pt} F \mathrm{~N}$ C H

$\begin{array}{lllll}90 & 1 & 4 & 20 & 12\end{array}$

Direct

$\begin{array}{llll}0.7999999999999972 & 0.6666669999999968 & 0.0000000000000000\end{array}$

$\begin{array}{llll}0.9000040000000027 & 0.8333319999999986 & 0.0000000000000000\end{array}$

$\begin{array}{lllll}0.7999999999999972 & 0.3333330000000032 & 0.0000000000000000\end{array}$

$\begin{array}{llll}0.9000040000000027 & 0.4999990000000025 & 0.0000000000000000\end{array}$

$\begin{array}{llll}0.7999999999999972 & 0.0000000000000000 & 0.0000000000000000\end{array}$

$\begin{array}{llll}0.9000040000000027 & 0.1666650000000018 & 0.0000000000000000\end{array}$

$\begin{array}{llll}0.6000090000000000 & 0.6666669999999968 & 0.0000000000000000\end{array}$

$\begin{array}{llll}0.7000140000000030 & 0.8333319999999986 & 0.0000000000000000\end{array}$

$\begin{array}{lllll}0.3999999999999986 & 0.6666669999999968 & 0.0000000000000000\end{array}$

$\begin{array}{lllll}0.5000039999999970 & 0.8333319999999986 & 0.0000000000000000\end{array}$

$\begin{array}{llll}0.6000090000000000 & 0.3333330000000032 & 0.0000000000000000\end{array}$

$\begin{array}{llll}0.7000140000000030 & 0.4999990000000025 & 0.0000000000000000\end{array}$

$\begin{array}{lllll}0.3999999999999986 & 0.3333330000000032 & 0.0000000000000000\end{array}$

$\begin{array}{llll}0.5000039999999970 & 0.4999990000000025 & 0.0000000000000000\end{array}$

$\begin{array}{llll}0.6000090000000000 & 0.0000000000000000 & 0.0000000000000000\end{array}$ 
$\begin{array}{lll}0.7000140000000030 & 0.1666650000000018 & 0.0000000000000000\end{array}$

$\begin{array}{llll}0.3999999999999986 & 0.0000000000000000 & 0.0000000000000000\end{array}$

$\begin{array}{llll}0.5000039999999970 & 0.1666650000000018 & 0.0000000000000000\end{array}$

$\begin{array}{llll}0.2000090000000014 & 0.6666669999999968 & 0.0000000000000000\end{array}$

$\begin{array}{llll}0.3000139999999973 & 0.8333319999999986 & 0.0000000000000000\end{array}$

$\begin{array}{llll}0.0000000000000000 & 0.6666669999999968 & 0.0000000000000000\end{array}$

$\begin{array}{lllll}0.1000039999999984 & 0.8333319999999986 & 0.0000000000000000\end{array}$

$\begin{array}{llll}0.2000090000000014 & 0.3333330000000032 & 0.0000000000000000\end{array}$

$\begin{array}{llll}0.3000139999999973 & 0.4999990000000025 & 0.0000000000000000\end{array}$

$\begin{array}{llll}0.0000000000000000 & 0.3333330000000032 & 0.0000000000000000\end{array}$

$\begin{array}{lll}0.1000039999999984 & 0.4999990000000025 & 0.0000000000000000\end{array}$

$\begin{array}{llll}0.2000090000000014 & 0.0000000000000000 & 0.0000000000000000\end{array}$

$\begin{array}{llll}0.3000139999999973 & 0.1666650000000018 & 0.0000000000000000\end{array}$

$\begin{array}{llll}0.0000000000000000 & 0.0000000000000000 & 0.0000000000000000\end{array}$

$\begin{array}{llll}0.1000039999999984 & 0.1666650000000018 & 0.0000000000000000\end{array}$

$\begin{array}{lllll}0.9000040000000027 & 0.7222220000000021 & 0.0666289999999989\end{array}$

$\begin{array}{lllll}0.7999999999999972 & 0.8888869999999969 & 0.0666289999999989\end{array}$

$\begin{array}{lllll}0.9000040000000027 & 0.3888880000000015 & 0.0666289999999989\end{array}$

$\begin{array}{llll}0.7999999999999972 & 0.5555540000000008 & 0.0666289999999989\end{array}$

$\begin{array}{llll}0.9000040000000027 & 0.0555549999999982 & 0.0666289999999989\end{array}$

$\begin{array}{lllll}0.7999999999999972 & 0.2222209999999976 & 0.0666289999999989\end{array}$

$\begin{array}{llll}0.6000090000000000 & 0.8888869999999969 & 0.0666289999999989\end{array}$

$\begin{array}{llll}0.7000140000000030 & 0.7222220000000021 & 0.0666289999999989\end{array}$

$\begin{array}{lllll}0.5000039999999970 & 0.7222220000000021 & 0.0666289999999989\end{array}$

$\begin{array}{llll}0.3999999999999986 & 0.8888869999999969 & 0.0666289999999989\end{array}$

$\begin{array}{lllll}0.6000090000000000 & 0.5555540000000008 & 0.0666289999999989\end{array}$

$\begin{array}{llll}0.7000140000000030 & 0.3888880000000015 & 0.0666289999999989\end{array}$

$\begin{array}{llll}0.5000039999999970 & 0.3888880000000015 & 0.0666289999999989\end{array}$

$\begin{array}{llll}0.3999999999999986 & 0.5555540000000008 & 0.0666289999999989\end{array}$

$\begin{array}{llll}0.6000090000000000 & 0.2222209999999976 & 0.0666289999999989\end{array}$

$\begin{array}{lllll}0.7000140000000030 & 0.0555549999999982 & 0.0666289999999989\end{array}$

$\begin{array}{lllll}0.5000039999999970 & 0.0555549999999982 & 0.0666289999999989\end{array}$

$\begin{array}{lllll}0.3999999999999986 & 0.2222209999999976 & 0.0666289999999989\end{array}$

$\begin{array}{llll}0.2000090000000014 & 0.8888869999999969 & 0.0666289999999989\end{array}$

$\begin{array}{llll}0.3000139999999973 & 0.7222220000000021 & 0.0666289999999989\end{array}$

$\begin{array}{lllll}0.1000039999999984 & 0.7222220000000021 & 0.0666289999999989\end{array}$

$\begin{array}{llll}0.0000000000000000 & 0.8888869999999969 & 0.0666289999999989\end{array}$

$\begin{array}{llll}0.2000090000000014 & 0.5555540000000008 & 0.0666289999999989\end{array}$

$\begin{array}{llll}0.3000139999999973 & 0.3888880000000015 & 0.0666289999999989\end{array}$

$\begin{array}{llll}0.1000039999999984 & 0.3888880000000015 & 0.0666289999999989\end{array}$

$\begin{array}{llll}0.0000000000000000 & 0.5555540000000008 & 0.0666289999999989\end{array}$

$\begin{array}{lllll}0.2000090000000014 & 0.2222209999999976 & 0.0666289999999989\end{array}$

$\begin{array}{llll}0.3000139999999973 & 0.0555549999999982 & 0.0666289999999989\end{array}$

$\begin{array}{lllll}0.1000039999999984 & 0.0555549999999982 & 0.0666289999999989\end{array}$

$\begin{array}{lllll}0.0000000000000000 & 0.2222209999999976 & 0.0666289999999989\end{array}$

$\begin{array}{llll}0.8006816318489818 & 0.7787809526342435 & 0.1342519077699589\end{array}$

$\begin{array}{llll}0.9000527846427597 & 0.9448698533128714 & 0.1340536849569539\end{array}$ 
$\begin{array}{llll}0.8011610199979179 & 0.4458536912288192 & 0.1342722246570375\end{array}$

$\begin{array}{llll}0.9000773240912883 & 0.6120974707391155 & 0.1345639565978817\end{array}$

$\begin{array}{llll}0.8012781971423266 & 0.1117117302325265 & 0.1343280889800210\end{array}$

$\begin{array}{llll}0.8999855943105186 & 0.2786350310386028 & 0.1346216500276045\end{array}$

$\begin{array}{llll}0.4002288160076318 & 0.7800937523669098 & 0.1337892093250400\end{array}$

$\begin{array}{llll}0.6016361472662218 & 0.7793064429662994 & 0.1337921359351454\end{array}$

$\begin{array}{llll}0.7007107221608074 & 0.9450194540970528 & 0.1342339697979011\end{array}$

$\begin{array}{llll}0.5004150087226140 & 0.9463796007261550 & 0.1336656216456235\end{array}$

$\begin{array}{llll}0.4001148661107334 & 0.4473239359258743 & 0.1377971483434514\end{array}$

$\begin{array}{llll}0.6019092365161078 & 0.4468880846389864 & 0.1326824849814646\end{array}$

$\begin{array}{llll}0.7016786996923171 & 0.6126691661025631 & 0.1338569057219625\end{array}$

$\begin{array}{llll}0.5012041387666454 & 0.6130741228420360 & 0.1329767722342406\end{array}$

$\begin{array}{llll}0.4000161178906553 & 0.1189656942090239 & 0.1422842028935961\end{array}$

$\begin{array}{llll}0.6026962462276392 & 0.1109153998707772 & 0.1327264975437938\end{array}$

$\begin{array}{llll}0.7028903198990313 & 0.2789629742429580 & 0.1336811727362236\end{array}$

$\begin{array}{llll}0.5030765676164409 & 0.2825865315005525 & 0.1324164362064247\end{array}$

$\begin{array}{llll}0.9994841524199239 & 0.7787493658406025 & 0.1341994475202916\end{array}$

$\begin{array}{llll}0.1986685422956203 & 0.7792687959387020 & 0.1337798705671228\end{array}$

$\begin{array}{llll}0.2998339024505483 & 0.9463397240864678 & 0.1336509978684529\end{array}$

$\begin{array}{llll}0.0994485025640692 & 0.9449414674424518 & 0.1342035172654837\end{array}$

$\begin{array}{llll}0.9989060758431895 & 0.4458809522098249 & 0.1342701284656999\end{array}$

$\begin{array}{lllll}0.1982306601957925 & 0.4468730382663208 & 0.1326435165083948\end{array}$

$\begin{array}{lllll}0.2994143155708994 & 0.6130123500825541 & 0.1328891159709287\end{array}$

$\begin{array}{llll}0.0987232568274078 & 0.6126183275382114 & 0.1339136935227122\end{array}$

$\begin{array}{llll}0.9987782856178640 & 0.1116940481081605 & 0.1343427329522725\end{array}$

$\begin{array}{lllll}0.1973223721951878 & 0.1108384649905154 & 0.1327270515646984\end{array}$

$\begin{array}{llll}0.2969430812053354 & 0.2825587912764156 & 0.1324249747202506\end{array}$

$\begin{array}{llll}0.0970499634852157 & 0.2789680020193603 & 0.1335965841257119\end{array}$

$\begin{array}{llll}0.3986696117965395 & 0.4419009326806526 & 0.2123174012133726\end{array}$

$\begin{array}{llll}0.3976871110446217 & 0.5788650362804563 & 0.2258789582444827\end{array}$

$\begin{array}{llll}0.2565909637284524 & 0.4386356398312725 & 0.2266801383652159\end{array}$

$\begin{array}{llll}0.3995082913705547 & 0.2990682184377008 & 0.2176240571091095\end{array}$

$\begin{array}{llll}0.5407037634151153 & 0.4397373541562705 & 0.2267573686513003\end{array}$

$\begin{array}{llll}0.2287497590535941 & 0.2741990116177467 & 0.2201100162749675\end{array}$

$\begin{array}{lllll}0.5685524440337997 & 0.6047375413330427 & 0.2275850558393618\end{array}$

$\begin{array}{llll}0.3212655748505880 & 0.2449558037395106 & 0.2134649410701411\end{array}$

$\begin{array}{llll}0.4753373293242447 & 0.6348949431622202 & 0.2252136396464359\end{array}$

$\begin{array}{llll}0.4782884090590638 & 0.2454809946998088 & 0.2138033194862274\end{array}$

$\begin{array}{llll}0.1994177869505104 & 0.3629578253807429 & 0.2274461993743842\end{array}$

$\begin{array}{llll}0.1978217035146179 & 0.5129722986317455 & 0.2305809108993019\end{array}$

$\begin{array}{llll}0.5985284904167050 & 0.5148265709139057 & 0.2300108076831617\end{array}$

$\begin{array}{llll}0.3194428599145482 & 0.6342633988906295 & 0.2257855077186275\end{array}$

$\begin{array}{llll}0.5988793373412532 & 0.3647169556878779 & 0.2279742245223399\end{array}$

$\begin{array}{lllll}0.2266592598532831 & 0.6032452152546560 & 0.2286552016830509\end{array}$

$\begin{array}{llll}0.5703906395497963 & 0.2756045139930813 & 0.2207982039910922\end{array}$

$\begin{array}{llll}0.3504230747471564 & 0.1510428100708062 & 0.2065077697272237\end{array}$

$\begin{array}{lllll}0.4499734036709184 & 0.1513933878417310 & 0.2066644814688507\end{array}$ 
$\begin{array}{llll}0.1028975141235040 & 0.3904814072961312 & 0.2334060668054716\end{array}$ $\begin{array}{llll}0.6949053099856554 & 0.4857983579568373 & 0.2346079154105321\end{array}$

$\begin{array}{llll}0.4451487410040606 & 0.7283380087905222 & 0.2240587495227006\end{array}$

$\begin{array}{lllll}0.3487617576703048 & 0.7279752839711521 & 0.2244326620597935\end{array}$

$\begin{array}{llll}0.1018306484524723 & 0.4828607446461786 & 0.2350263645834232\end{array}$

$\begin{array}{llll}0.6950468538026733 & 0.3933532674149344 & 0.2337044634205528\end{array}$

$\begin{array}{llll}0.1743014863638502 & 0.2229954148345364 & 0.2187906336703401\end{array}$

$\begin{array}{llll}0.6228316016720786 & 0.6562180570293838 & 0.2279896324721182\end{array}$

$\begin{array}{llll}0.6253845489437982 & 0.2249079381079231 & 0.2196665015025492\end{array}$

$\begin{array}{llll}0.1719247856547914 & 0.6542398196036620 & 0.2293505145834361\end{array}$

$\begin{array}{llll}0.3053612258206467 & 0.0934559930670403 & 0.2115092814988856\end{array}$

$\begin{array}{llll}0.4953769972171579 & 0.0940860979236138 & 0.2117188849690495\end{array}$

$\begin{array}{llll}0.3016123745621118 & 0.7852511267268639 & 0.2238743767043525\end{array}$

$\begin{array}{llll}0.4917261577106679 & 0.7859748816010099 & 0.2233062290775170\end{array}$

$\begin{array}{llll}0.7543175135215799 & 0.5307844454456078 & 0.2374910568109603\end{array}$

$\begin{array}{llll}0.0438513357831527 & 0.3447011788796090 & 0.2350082819430191\end{array}$

$\begin{array}{llll}0.0418903750583646 & 0.5270824805422277 & 0.2382501157246723\end{array}$

$\begin{array}{llll}0.7545714276624570 & 0.3482260505664829 & 0.2355713609309728\end{array}$

FeP-Pt(111) - Hhcp-0, vdW-DF-revPBE:

$\mathrm{Pt} \mathrm{Fe} \mathrm{N} \mathrm{C} \mathrm{H}$

1.00000000000000

$\begin{array}{lll}14.2799999999999994 & 0.0000000000000000 & 0.0000000000000000\end{array}$

$\begin{array}{llll}0.0000000000000000 & 14.8409999999999993 & 0.0000000000000000\end{array}$

$\begin{array}{llll}0.0000000000000000 & 0.0000000000000000 & 35.0000000000000000\end{array}$

$\mathrm{Pt} \mathrm{Fe} \mathrm{N} \mathrm{C}$

$\begin{array}{lllll}90 & 1 & 4 & 20 & 12\end{array}$

Direct

$\begin{array}{llll}0.7999999999999972 & 0.6666669999999968 & 0.0000000000000000\end{array}$

$\begin{array}{llll}0.9000040000000027 & 0.8333319999999986 & 0.0000000000000000\end{array}$

$\begin{array}{llll}0.7999999999999972 & 0.3333330000000032 & 0.0000000000000000\end{array}$

$\begin{array}{llll}0.9000040000000027 & 0.4999990000000025 & 0.0000000000000000\end{array}$

$\begin{array}{llll}0.7999999999999972 & 0.0000000000000000 & 0.0000000000000000\end{array}$

$\begin{array}{llll}0.9000040000000027 & 0.1666650000000018 & 0.0000000000000000\end{array}$

$\begin{array}{llll}0.6000090000000000 & 0.6666669999999968 & 0.0000000000000000\end{array}$

$\begin{array}{llll}0.7000140000000030 & 0.8333319999999986 & 0.0000000000000000\end{array}$

$\begin{array}{llll}0.3999999999999986 & 0.6666669999999968 & 0.0000000000000000\end{array}$

$\begin{array}{llll}0.5000039999999970 & 0.8333319999999986 & 0.0000000000000000\end{array}$

$\begin{array}{llll}0.6000090000000000 & 0.3333330000000032 & 0.0000000000000000\end{array}$

$\begin{array}{llll}0.7000140000000030 & 0.4999990000000025 & 0.0000000000000000\end{array}$

$\begin{array}{llll}0.3999999999999986 & 0.3333330000000032 & 0.0000000000000000\end{array}$

$\begin{array}{llll}0.5000039999999970 & 0.4999990000000025 & 0.0000000000000000\end{array}$

$\begin{array}{llll}0.6000090000000000 & 0.0000000000000000 & 0.0000000000000000\end{array}$

$\begin{array}{llll}0.7000140000000030 & 0.1666650000000018 & 0.0000000000000000\end{array}$

$\begin{array}{llll}0.3999999999999986 & 0.0000000000000000 & 0.0000000000000000\end{array}$

$\begin{array}{llll}0.5000039999999970 & 0.1666650000000018 & 0.0000000000000000\end{array}$

$\begin{array}{llll}0.2000090000000014 & 0.6666669999999968 & 0.0000000000000000\end{array}$ 
$\begin{array}{lll}0.3000139999999973 & 0.8333319999999986 & 0.0000000000000000\end{array}$ $\begin{array}{lllll}0.0000000000000000 & 0.6666669999999968 & 0.0000000000000000\end{array}$ $\begin{array}{llll}0.1000039999999984 & 0.8333319999999986 & 0.0000000000000000\end{array}$ $\begin{array}{llll}0.2000090000000014 & 0.3333330000000032 & 0.0000000000000000\end{array}$ $\begin{array}{llll}0.3000139999999973 & 0.4999990000000025 & 0.0000000000000000\end{array}$ $\begin{array}{llll}0.0000000000000000 & 0.3333330000000032 & 0.0000000000000000\end{array}$ $\begin{array}{lll}0.1000039999999984 & 0.4999990000000025 & 0.0000000000000000\end{array}$ $\begin{array}{llll}0.2000090000000014 & 0.0000000000000000 & 0.0000000000000000\end{array}$ $\begin{array}{llll}0.3000139999999973 & 0.1666650000000018 & 0.0000000000000000\end{array}$ $\begin{array}{llll}0.0000000000000000 & 0.0000000000000000 & 0.0000000000000000\end{array}$ $\begin{array}{llll}0.1000039999999984 & 0.1666650000000018 & 0.0000000000000000\end{array}$ $\begin{array}{lllll}0.9000040000000027 & 0.7222220000000021 & 0.0666289999999989\end{array}$ $\begin{array}{llll}0.7999999999999972 & 0.8888869999999969 & 0.0666289999999989\end{array}$ $\begin{array}{lllll}0.9000040000000027 & 0.3888880000000015 & 0.0666289999999989\end{array}$ $\begin{array}{llll}0.7999999999999972 & 0.5555540000000008 & 0.0666289999999989\end{array}$ $\begin{array}{llll}0.9000040000000027 & 0.0555549999999982 & 0.0666289999999989\end{array}$ $\begin{array}{lllll}0.7999999999999972 & 0.2222209999999976 & 0.0666289999999989\end{array}$ $\begin{array}{llll}0.6000090000000000 & 0.8888869999999969 & 0.0666289999999989\end{array}$ $\begin{array}{llll}0.7000140000000030 & 0.7222220000000021 & 0.0666289999999989\end{array}$ $\begin{array}{lllll}0.5000039999999970 & 0.7222220000000021 & 0.0666289999999989\end{array}$ $\begin{array}{llll}0.3999999999999986 & 0.8888869999999969 & 0.0666289999999989\end{array}$ $\begin{array}{llll}0.6000090000000000 & 0.5555540000000008 & 0.0666289999999989\end{array}$ $\begin{array}{llll}0.7000140000000030 & 0.3888880000000015 & 0.0666289999999989\end{array}$ $\begin{array}{llll}0.5000039999999970 & 0.3888880000000015 & 0.0666289999999989\end{array}$ $\begin{array}{llll}0.3999999999999986 & 0.5555540000000008 & 0.0666289999999989\end{array}$ $\begin{array}{lllll}0.6000090000000000 & 0.2222209999999976 & 0.0666289999999989\end{array}$ $\begin{array}{llll}0.7000140000000030 & 0.0555549999999982 & 0.0666289999999989\end{array}$ $\begin{array}{lllll}0.5000039999999970 & 0.0555549999999982 & 0.0666289999999989\end{array}$ $\begin{array}{lllll}0.3999999999999986 & 0.2222209999999976 & 0.0666289999999989\end{array}$ $\begin{array}{llll}0.2000090000000014 & 0.8888869999999969 & 0.0666289999999989\end{array}$ $\begin{array}{lllll}0.3000139999999973 & 0.7222220000000021 & 0.0666289999999989\end{array}$ $\begin{array}{lllll}0.1000039999999984 & 0.7222220000000021 & 0.0666289999999989\end{array}$ $\begin{array}{llll}0.0000000000000000 & 0.8888869999999969 & 0.0666289999999989\end{array}$ $\begin{array}{llll}0.2000090000000014 & 0.5555540000000008 & 0.0666289999999989\end{array}$ $\begin{array}{llll}0.3000139999999973 & 0.3888880000000015 & 0.0666289999999989\end{array}$ $\begin{array}{lllll}0.1000039999999984 & 0.3888880000000015 & 0.0666289999999989\end{array}$ $\begin{array}{llll}0.0000000000000000 & 0.5555540000000008 & 0.0666289999999989\end{array}$ $\begin{array}{llll}0.2000090000000014 & 0.2222209999999976 & 0.0666289999999989\end{array}$ $\begin{array}{llll}0.3000139999999973 & 0.0555549999999982 & 0.0666289999999989\end{array}$ $\begin{array}{lllll}0.1000039999999984 & 0.0555549999999982 & 0.0666289999999989\end{array}$ $\begin{array}{lllll}0.0000000000000000 & 0.2222209999999976 & 0.0666289999999989\end{array}$ $\begin{array}{llll}0.8014220552749698 & 0.7790708972215939 & 0.1335113926011993\end{array}$ $\begin{array}{llll}0.8998623652815406 & 0.9462195224009594 & 0.1341906125814359\end{array}$ $\begin{array}{llll}0.8016109864226791 & 0.4442242042690211 & 0.1342619007141117\end{array}$ $\begin{array}{llll}0.8999227546003926 & 0.6114230446455792 & 0.1344734269915535\end{array}$ $\begin{array}{llll}0.8005789642250338 & 0.1123604195916114 & 0.1343301534463292\end{array}$ $\begin{array}{llll}0.9002810283259264 & 0.2778784425678979 & 0.1341635029296489\end{array}$ 
$\begin{array}{llll}0.3992166098827155 & 0.7792719117914430 & 0.1347489181412868\end{array}$ $\begin{array}{llll}0.6015250241862405 & 0.7802281671639780 & 0.1379071344947320\end{array}$ $\begin{array}{llll}0.7015362297112283 & 0.9471185312252299 & 0.1337120472137150\end{array}$ $\begin{array}{llll}0.5009241146620592 & 0.9489447612730671 & 0.1326549866524147\end{array}$ $\begin{array}{llll}0.4005448443887559 & 0.4446946476125304 & 0.1354461076845701\end{array}$ $\begin{array}{llll}0.6023237955083399 & 0.4433730304304668 & 0.1330581748141793\end{array}$ $\begin{array}{llll}0.7025012158641438 & 0.6108363495650524 & 0.1329026538112150\end{array}$ $\begin{array}{llll}0.5004267890407627 & 0.6082405794873340 & 0.1319945429811980\end{array}$ $\begin{array}{llll}0.4003153852660485 & 0.1132471169873371 & 0.1345450792649095\end{array}$ $\begin{array}{llll}0.6009741894153661 & 0.1126833042058321 & 0.1343603637951103\end{array}$ $\begin{array}{llll}0.7009250655733352 & 0.2778890413296148 & 0.1339232982922542\end{array}$ $\begin{array}{llll}0.5004252128808342 & 0.2785927943274869 & 0.1338785032794310\end{array}$ $\begin{array}{llll}0.9982149892036591 & 0.7791033321402880 & 0.1334164855046838\end{array}$ $\begin{array}{llll}0.1976674139842690 & 0.7805340340589295 & 0.1375500483776237\end{array}$ $\begin{array}{llll}0.2990697639829616 & 0.9490874956073700 & 0.1326639189978972\end{array}$ $\begin{array}{lllll}0.0982810732376893 & 0.9474744892668456 & 0.1336041299554132\end{array}$ $\begin{array}{llll}0.9989481513716852 & 0.4440287023201435 & 0.1341706656869179\end{array}$ $\begin{array}{llll}0.1983021448592842 & 0.4432534672741160 & 0.1330247417972124\end{array}$ $\begin{array}{llll}0.2997365971462890 & 0.6079454685095830 & 0.1321086661327988\end{array}$ $\begin{array}{llll}0.0974259274424951 & 0.6107115921990848 & 0.1328826693507077\end{array}$ $\begin{array}{llll}0.9996629516208736 & 0.1124630683629281 & 0.1343708543271043\end{array}$ $\begin{array}{lllll}0.1995959137924604 & 0.1128338916829441 & 0.1344332147729936\end{array}$ $\begin{array}{llll}0.3006938964084844 & 0.2786363084878632 & 0.1339038186850383\end{array}$ $\begin{array}{llll}0.0999751747550919 & 0.2777198521076613 & 0.1339698906815888\end{array}$ $\begin{array}{llll}0.3962812474130840 & 0.5449716371489371 & 0.2091202399491835\end{array}$ $\begin{array}{lllll}0.4985324908757710 & 0.6505477311041896 & 0.2152719858201202\end{array}$ $\begin{array}{llll}0.2944775286873371 & 0.6502843590291049 & 0.2154644282449198\end{array}$ $\begin{array}{llll}0.2951046235982643 & 0.4534758902916420 & 0.2279787682215027\end{array}$ $\begin{array}{llll}0.4972470838405905 & 0.4535939405618961 & 0.2276540240934327\end{array}$ $\begin{array}{llll}0.1552547721334210 & 0.5521306511839824 & 0.2270864282472514\end{array}$ $\begin{array}{lllll}0.6374497037126587 & 0.5521133357380672 & 0.2266832465317279\end{array}$ $\begin{array}{llll}0.1991122865516390 & 0.4679661593031598 & 0.2291580986960106\end{array}$ $\begin{array}{llll}0.5956954825484889 & 0.6368114919271465 & 0.2204026865046727\end{array}$ $\begin{array}{llll}0.3080634247770600 & 0.3611932171029300 & 0.2300766211749734\end{array}$ $\begin{array}{lllll}0.1971369031662433 & 0.6366636465452927 & 0.2204366161151080\end{array}$ $\begin{array}{llll}0.3072199425040921 & 0.7384184684865889 & 0.2066814956875787\end{array}$ $\begin{array}{lllll}0.5933783246226384 & 0.4678373554180192 & 0.2285606231731097\end{array}$ $\begin{array}{llll}0.4859567463371119 & 0.7388907821103092 & 0.2067509420059573\end{array}$ $\begin{array}{llll}0.4837610926708987 & 0.3613166634943571 & 0.2299848799830353\end{array}$ $\begin{array}{llll}0.3965668410563895 & 0.7809870764608200 & 0.1987440888512796\end{array}$ $\begin{array}{llll}0.3959283658676206 & 0.3185469563432118 & 0.2307176700378236\end{array}$ $\begin{array}{llll}0.1504569552615503 & 0.3819701651308958 & 0.2318206994760726\end{array}$ $\begin{array}{llll}0.2176139627936030 & 0.3161191868456103 & 0.2321081618023894\end{array}$ $\begin{array}{llll}0.1487388873793208 & 0.7190605113320035 & 0.2143050485412671\end{array}$ $\begin{array}{llll}0.6414448202066824 & 0.3815254613675583 & 0.2312770871456725\end{array}$ $\begin{array}{llll}0.6442710645053743 & 0.7195279070346583 & 0.2148482133000841\end{array}$ $\begin{array}{llll}0.5772305269170855 & 0.7853907528549245 & 0.2037109324171261\end{array}$ 
$\begin{array}{llll}0.2162046343898749 & 0.7850212072638927 & 0.2032670933088397\end{array}$

$\begin{array}{llll}0.5739138325328696 & 0.3159603493227008 & 0.2319975889538732\end{array}$

$\begin{array}{llll}0.0790721800605370 & 0.5519413806164764 & 0.2285281481740741\end{array}$

$\begin{array}{llll}0.7136047935040551 & 0.5516720159393521 & 0.2282836553007156\end{array}$

$\begin{array}{llll}0.3959714984061796 & 0.2453108680311688 & 0.2320447845723008\end{array}$

$\begin{array}{llll}0.3963038335802282 & 0.8540413742380830 & 0.2025218008296680\end{array}$

$\begin{array}{llll}0.0750081642762974 & 0.3738445893069173 & 0.2320861447334031\end{array}$

$\begin{array}{llll}0.2077360660008838 & 0.2437236251565957 & 0.2328120183901365\end{array}$

$\begin{array}{llll}0.5853344018695038 & 0.8576034894198606 & 0.2077628718638048\end{array}$

$\begin{array}{llll}0.7188228177785047 & 0.7298586472201679 & 0.2185051581319039\end{array}$

$\begin{array}{llll}0.7168479558606231 & 0.3729987734415787 & 0.2316154528180701\end{array}$

$\begin{array}{llll}0.0742216613029193 & 0.7298118005797805 & 0.2178572799166114\end{array}$

$\begin{array}{lllll}0.2082394119824623 & 0.8572071448447031 & 0.2075146931878763\end{array}$

$\begin{array}{llll}0.5834490960398291 & 0.2435245687709720 & 0.2329548554664376\end{array}$

FeP-Pt(111) - Hhcp-45, High Spin, vdW-DF-revPBE:

Pt Fe N C H

1.00000000000000

$\begin{array}{llll}14.2799999999999994 & 0.0000000000000000 & 0.0000000000000000\end{array}$

$\begin{array}{lll}0.0000000000000000 & 14.8409999999999993 & 0.0000000000000000\end{array}$

$\begin{array}{llll}0.0000000000000000 & 0.0000000000000000 & 35.0000000000000000\end{array}$

Pt Fe N C H

$\begin{array}{lllll}90 & 1 & 4 & 20 & 12\end{array}$

Direct

$\begin{array}{llll}0.7999999999999972 & 0.6666669999999968 & 0.0000000000000000\end{array}$

$\begin{array}{lllll}0.9000040000000027 & 0.8333319999999986 & 0.0000000000000000\end{array}$

$\begin{array}{lllll}0.7999999999999972 & 0.3333330000000032 & 0.0000000000000000\end{array}$

$\begin{array}{llll}0.9000040000000027 & 0.4999990000000025 & 0.0000000000000000\end{array}$

$\begin{array}{llll}0.7999999999999972 & 0.0000000000000000 & 0.0000000000000000\end{array}$

$\begin{array}{llll}0.9000040000000027 & 0.1666650000000018 & 0.0000000000000000\end{array}$

$\begin{array}{llll}0.6000090000000000 & 0.6666669999999968 & 0.0000000000000000\end{array}$

$\begin{array}{llll}0.7000140000000030 & 0.8333319999999986 & 0.0000000000000000\end{array}$

$\begin{array}{llll}0.3999999999999986 & 0.6666669999999968 & 0.0000000000000000\end{array}$

$\begin{array}{llll}0.5000039999999970 & 0.8333319999999986 & 0.0000000000000000\end{array}$

$\begin{array}{llll}0.6000090000000000 & 0.3333330000000032 & 0.0000000000000000\end{array}$

$\begin{array}{llll}0.7000140000000030 & 0.4999990000000025 & 0.0000000000000000\end{array}$

$\begin{array}{llll}0.3999999999999986 & 0.3333330000000032 & 0.0000000000000000\end{array}$

$\begin{array}{llll}0.5000039999999970 & 0.4999990000000025 & 0.0000000000000000\end{array}$

$\begin{array}{llll}0.6000090000000000 & 0.0000000000000000 & 0.0000000000000000\end{array}$

$\begin{array}{lllll}0.7000140000000030 & 0.1666650000000018 & 0.0000000000000000\end{array}$

$\begin{array}{lllll}0.3999999999999986 & 0.0000000000000000 & 0.0000000000000000\end{array}$

$\begin{array}{llll}0.5000039999999970 & 0.1666650000000018 & 0.0000000000000000\end{array}$

0.20000900000000140 .66666699999999680 .0000000000000000

$\begin{array}{llll}0.3000139999999973 & 0.8333319999999986 & 0.0000000000000000\end{array}$

$\begin{array}{lllll}0.0000000000000000 & 0.6666669999999968 & 0.0000000000000000\end{array}$

$\begin{array}{lllll}0.1000039999999984 & 0.8333319999999986 & 0.0000000000000000\end{array}$

$\begin{array}{lllll}0.2000090000000014 & 0.3333330000000032 & 0.0000000000000000\end{array}$ 
$\begin{array}{lll}0.3000139999999973 & 0.4999990000000025 & 0.0000000000000000\end{array}$

$\begin{array}{llll}0.0000000000000000 & 0.3333330000000032 & 0.0000000000000000\end{array}$

$\begin{array}{llll}0.1000039999999984 & 0.4999990000000025 & 0.0000000000000000\end{array}$

$\begin{array}{llll}0.2000090000000014 & 0.0000000000000000 & 0.0000000000000000\end{array}$

$\begin{array}{llll}0.3000139999999973 & 0.1666650000000018 & 0.0000000000000000\end{array}$

$\begin{array}{llll}0.0000000000000000 & 0.0000000000000000 & 0.0000000000000000\end{array}$

$\begin{array}{lllll}0.1000039999999984 & 0.1666650000000018 & 0.0000000000000000\end{array}$

$\begin{array}{llll}0.9000040000000027 & 0.7222220000000021 & 0.0666289999999989\end{array}$

$\begin{array}{lllll}0.7999999999999972 & 0.8888869999999969 & 0.0666289999999989\end{array}$

$\begin{array}{llll}0.9000040000000027 & 0.3888880000000015 & 0.0666289999999989\end{array}$

$\begin{array}{llll}0.7999999999999972 & 0.5555540000000008 & 0.0666289999999989\end{array}$

$\begin{array}{lllll}0.9000040000000027 & 0.0555549999999982 & 0.0666289999999989\end{array}$

$\begin{array}{lllll}0.7999999999999972 & 0.2222209999999976 & 0.0666289999999989\end{array}$

$\begin{array}{llll}0.6000090000000000 & 0.8888869999999969 & 0.0666289999999989\end{array}$

$\begin{array}{llll}0.7000140000000030 & 0.7222220000000021 & 0.0666289999999989\end{array}$

$\begin{array}{lllll}0.5000039999999970 & 0.7222220000000021 & 0.0666289999999989\end{array}$

$\begin{array}{lllll}0.3999999999999986 & 0.8888869999999969 & 0.0666289999999989\end{array}$

$\begin{array}{llll}0.6000090000000000 & 0.5555540000000008 & 0.0666289999999989\end{array}$

$\begin{array}{llll}0.7000140000000030 & 0.3888880000000015 & 0.0666289999999989\end{array}$

$\begin{array}{llll}0.5000039999999970 & 0.3888880000000015 & 0.0666289999999989\end{array}$

$\begin{array}{lllll}0.3999999999999986 & 0.5555540000000008 & 0.0666289999999989\end{array}$

$\begin{array}{lllll}0.6000090000000000 & 0.2222209999999976 & 0.0666289999999989\end{array}$

$\begin{array}{llll}0.7000140000000030 & 0.0555549999999982 & 0.0666289999999989\end{array}$

$\begin{array}{lllll}0.5000039999999970 & 0.0555549999999982 & 0.0666289999999989\end{array}$

$\begin{array}{lllll}0.3999999999999986 & 0.2222209999999976 & 0.0666289999999989\end{array}$

$\begin{array}{lllll}0.2000090000000014 & 0.8888869999999969 & 0.0666289999999989\end{array}$

$\begin{array}{lllll}0.3000139999999973 & 0.7222220000000021 & 0.0666289999999989\end{array}$

$\begin{array}{lllll}0.1000039999999984 & 0.7222220000000021 & 0.0666289999999989\end{array}$

$\begin{array}{llll}0.0000000000000000 & 0.8888869999999969 & 0.0666289999999989\end{array}$

$\begin{array}{llll}0.2000090000000014 & 0.5555540000000008 & 0.0666289999999989\end{array}$

$\begin{array}{llll}0.3000139999999973 & 0.3888880000000015 & 0.0666289999999989\end{array}$

$\begin{array}{lllll}0.1000039999999984 & 0.3888880000000015 & 0.0666289999999989\end{array}$

$\begin{array}{lllll}0.0000000000000000 & 0.5555540000000008 & 0.0666289999999989\end{array}$

$\begin{array}{lllll}0.2000090000000014 & 0.2222209999999976 & 0.0666289999999989\end{array}$

$\begin{array}{llll}0.3000139999999973 & 0.0555549999999982 & 0.0666289999999989\end{array}$

$\begin{array}{lllll}0.1000039999999984 & 0.0555549999999982 & 0.0666289999999989\end{array}$

$\begin{array}{lllll}0.0000000000000000 & 0.2222209999999976 & 0.0666289999999989\end{array}$

$\begin{array}{llll}0.8005253022532708 & 0.7769650304931779 & 0.1342522472025482\end{array}$

$\begin{array}{llll}0.9000891234108939 & 0.9420738320440816 & 0.1340982467239248\end{array}$

$\begin{array}{llll}0.8006910350499209 & 0.4425720592418032 & 0.1338453812754068\end{array}$

$\begin{array}{llll}0.9002462478282054 & 0.6102042408217316 & 0.1339519481475855\end{array}$

$\begin{array}{llll}0.8004678930131369 & 0.1082079110246378 & 0.1340478794595035\end{array}$

$\begin{array}{llll}0.8992935711700859 & 0.2750753976202461 & 0.1341066710015105\end{array}$

$\begin{array}{llll}0.4001370035081170 & 0.7784308334511402 & 0.1326634525909028\end{array}$

$\begin{array}{llll}0.6005459123183101 & 0.7768211452652705 & 0.1343671736816943\end{array}$

$\begin{array}{llll}0.7008282042053718 & 0.9423250165655617 & 0.1344541731599629\end{array}$

$\begin{array}{llll}0.5010813974107308 & 0.9417673081128632 & 0.1342480950307019\end{array}$ 
$\begin{array}{llll}0.4010015369468656 & 0.4488994741153952 & 0.1301447071192278\end{array}$ $\begin{array}{llll}0.6020662588649192 & 0.4416881605346802 & 0.1335430904375654\end{array}$ $\begin{array}{lllll}0.7016037583308573 & 0.6112160104361281 & 0.1334618122299853\end{array}$ $\begin{array}{llll}0.4996883677275406 & 0.6124871538971272 & 0.1351187254479483\end{array}$ $\begin{array}{llll}0.4003092261643097 & 0.1040648815128020 & 0.1332388811871739\end{array}$ $\begin{array}{llll}0.6012752787008867 & 0.1071285499435022 & 0.1339620880469372\end{array}$ $\begin{array}{llll}0.7001544986510694 & 0.2739682273450968 & 0.1336987480704276\end{array}$ $\begin{array}{llll}0.4990115014177052 & 0.2716545703209239 & 0.1380420674403129\end{array}$ $\begin{array}{llll}0.9998039286902510 & 0.7769150822503400 & 0.1342853219579312\end{array}$ $\begin{array}{llll}0.1996642640558405 & 0.7770116025315003 & 0.1341994687900059\end{array}$ $\begin{array}{llll}0.2990054040409797 & 0.9418044115781719 & 0.1343273177352961\end{array}$ $\begin{array}{llll}0.0992114801130661 & 0.9420652490846831 & 0.1344661248961750\end{array}$ $\begin{array}{llll}0.9996461389142035 & 0.4420836024095208 & 0.1334182571124513\end{array}$ $\begin{array}{llll}0.2001884156198186 & 0.4376914889450739 & 0.1376249408708037\end{array}$ $\begin{array}{llll}0.3006537752460659 & 0.6125138041272477 & 0.1348046184966299\end{array}$ $\begin{array}{llll}0.0995620088161132 & 0.6106247842584628 & 0.1326750602042617\end{array}$ $\begin{array}{llll}0.9994924016389871 & 0.1077879720361310 & 0.1341649443123503\end{array}$ $\begin{array}{llll}0.1987602334867702 & 0.1064676658840411 & 0.1340380922687343\end{array}$ $\begin{array}{llll}0.3023543172137421 & 0.2702970584941931 & 0.1361902947095948\end{array}$ $\begin{array}{llll}0.0979834367396343 & 0.2728245426230018 & 0.1328959460186068\end{array}$ $\begin{array}{llll}0.3978412443751162 & 0.5830243039753880 & 0.2016557911816028\end{array}$ $\begin{array}{lllll}0.3986188157611554 & 0.7134975737618506 & 0.2248227938877971\end{array}$ $\begin{array}{llll}0.2538673366703972 & 0.5752692558074691 & 0.2192616986677215\end{array}$ $\begin{array}{llll}0.3961915045751921 & 0.4337781738447575 & 0.2079917741965391\end{array}$ $\begin{array}{llll}0.5404688355641604 & 0.5736388165511682 & 0.2207241662502554\end{array}$ $\begin{array}{llll}0.2231269362822043 & 0.4146077695486585 & 0.2022346960363242\end{array}$ $\begin{array}{llll}0.5701556156600703 & 0.7377008662558023 & 0.2256923639916275\end{array}$ $\begin{array}{llll}0.3185030955763332 & 0.3808914354031236 & 0.2030914867956099\end{array}$ $\begin{array}{llll}0.4766962010833741 & 0.7686724086223288 & 0.2270591353994923\end{array}$ $\begin{array}{llll}0.4753270677950226 & 0.3780743665024195 & 0.2079391115905037\end{array}$ $\begin{array}{lllll}0.1953649962747657 & 0.5028946297591421 & 0.2155653458639494\end{array}$ $\begin{array}{llll}0.1967387897893053 & 0.6496604952859409 & 0.2270228095861410\end{array}$ $\begin{array}{llll}0.5996864603216938 & 0.6477341251350555 & 0.2242932971542853\end{array}$ $\begin{array}{llll}0.3212562215726396 & 0.7689941351206361 & 0.2293497024240661\end{array}$ $\begin{array}{llll}0.5971666829341585 & 0.4987862699408802 & 0.2203129784648153\end{array}$ $\begin{array}{lllll}0.2278932601734603 & 0.7385648594090672 & 0.2302023215855135\end{array}$ $\begin{array}{lllll}0.5671940970381917 & 0.4090534738235007 & 0.2134243421377232\end{array}$ $\begin{array}{llll}0.3442376176070296 & 0.2858536084368523 & 0.1958335166083854\end{array}$ $\begin{array}{llll}0.4493773181245249 & 0.2848594800192744 & 0.1976201569221137\end{array}$ $\begin{array}{lllll}0.0990470086288110 & 0.5296163356981135 & 0.2221401220206091\end{array}$ $\begin{array}{llll}0.6962057313845311 & 0.6176590552649365 & 0.2258120341389470\end{array}$ $\begin{array}{llll}0.4476164301289388 & 0.8612504591290132 & 0.2323342890474499\end{array}$ $\begin{array}{llll}0.3511763821577182 & 0.8612570821013623 & 0.2338697821993999\end{array}$ $\begin{array}{llll}0.1000289942356477 & 0.6210693358491736 & 0.2289091074930942\end{array}$ $\begin{array}{llll}0.6946317750572569 & 0.5249741054328835 & 0.2242380023250596\end{array}$ $\begin{array}{llll}0.1705424486751750 & 0.3621873059013801 & 0.2064478750619479\end{array}$ $\begin{array}{lllll}0.6246966521985496 & 0.7886638832551681 & 0.2279000121852181\end{array}$ 
$\begin{array}{llll}0.6215683083023479 & 0.3576579241876325 & 0.2139058559671780\end{array}$

$\begin{array}{llll}0.1741496095795376 & 0.7897215356238889 & 0.2342618338802133\end{array}$

$\begin{array}{lllll}0.3045549364862818 & 0.2322484308411677 & 0.2098131492020913\end{array}$

$\begin{array}{llll}0.4847613342948583 & 0.2286304457265472 & 0.2115833460978153\end{array}$

$\begin{array}{llll}0.3045913475361626 & 0.9183417611719115 & 0.2374634164069691\end{array}$

$\begin{array}{llll}0.4946594292123399 & 0.9183726696494859 & 0.2344858127593314\end{array}$

$\begin{array}{llll}0.7566368892959501 & 0.6616061355042810 & 0.2279608120277002\end{array}$

$\begin{array}{llll}0.0391571570682555 & 0.4847967730957906 & 0.2209357535943560\end{array}$

$\begin{array}{llll}0.0408109553378960 & 0.6651334589715875 & 0.2341478041770224\end{array}$

$\begin{array}{llll}0.7533857167402348 & 0.4787364384748642 & 0.2243350164579868\end{array}$

FeP-Pt(111) - Hhcp-45, Intermediate Spin, vdW-DF-revPBE:

$\mathrm{Pt} \mathrm{Fe} \mathrm{N} \mathrm{C} \mathrm{H}$

1.00000000000000

$\begin{array}{lll}14.2799999999999994 & 0.0000000000000000 & 0.0000000000000000\end{array}$

$\begin{array}{lll}0.0000000000000000 & 14.8409999999999993 & 0.0000000000000000\end{array}$

$\begin{array}{llll}0.0000000000000000 & 0.0000000000000000 & 35.0000000000000000\end{array}$

$\mathrm{Pt} F \mathrm{~N}$ C $\mathrm{H}$

$\begin{array}{lllll}90 & 1 & 4 & 20 & 12\end{array}$

Direct

$\begin{array}{llll}0.0977929388823853 & 0.2703894479853659 & 0.1326193832865741\end{array}$

$\begin{array}{llll}0.3012654766353791 & 0.2707250976875528 & 0.1361888777797446\end{array}$

$\begin{array}{llll}0.1990104022478718 & 0.1040201524522715 & 0.1337289475663823\end{array}$

$\begin{array}{llll}0.9993865339327783 & 0.1044367119819682 & 0.1335961886117829\end{array}$

$\begin{array}{llll}0.0985511190051440 & 0.6071030296186564 & 0.1327478648738634\end{array}$

$\begin{array}{llll}0.2995797297030620 & 0.6099486627846389 & 0.1334469570098733\end{array}$

$\begin{array}{llll}0.1999297419541293 & 0.4374437283875281 & 0.1379557934072864\end{array}$

$\begin{array}{llll}0.9986432060980874 & 0.4391436021326811 & 0.1327195067736966\end{array}$

$\begin{array}{llll}0.0997627305151738 & 0.9381825732024041 & 0.1339560394791519\end{array}$

$\begin{array}{llll}0.2992703335924460 & 0.9391481344692352 & 0.1338836723807759\end{array}$

$\begin{array}{llll}0.1995835802294295 & 0.7734009937991857 & 0.1342190555276304\end{array}$

$\begin{array}{llll}0.9995956607312237 & 0.7731250839266792 & 0.1339589237393000\end{array}$

$\begin{array}{llll}0.4981456062180811 & 0.2723571908573632 & 0.1380156842044897\end{array}$

$\begin{array}{llll}0.7000211966417567 & 0.2717338051753870 & 0.1328163805332849\end{array}$

$\begin{array}{llll}0.6002817375164824 & 0.1053600814957534 & 0.1338181692687996\end{array}$

$\begin{array}{llll}0.4000214307645535 & 0.1029494802765711 & 0.1331608423288273\end{array}$

$\begin{array}{llll}0.5013149084690127 & 0.6105413674745748 & 0.1336814952128833\end{array}$

$\begin{array}{llll}0.7023305892999900 & 0.6077530762026200 & 0.1333645837779471\end{array}$

$\begin{array}{llll}0.6016810343253098 & 0.4402929405103038 & 0.1338078853598645\end{array}$

$\begin{array}{llll}0.4014537648215395 & 0.4464217094972298 & 0.1293783651409157\end{array}$

$\begin{array}{lllll}0.5007056347703127 & 0.9393676201204997 & 0.1338618100661719\end{array}$

$\begin{array}{llll}0.7004975519391081 & 0.9395927750175161 & 0.1338685015214480\end{array}$

$\begin{array}{llll}0.6009397899570885 & 0.7739597346360796 & 0.1343529801994094\end{array}$

$\begin{array}{llll}0.4003891073442958 & 0.7751689963098514 & 0.1328378501634546\end{array}$

$\begin{array}{llll}0.8991831417209326 & 0.2727384851813710 & 0.1341742789876790\end{array}$

$\begin{array}{llll}0.8002503537126984 & 0.1054147718386389 & 0.1336653410216186\end{array}$

$\begin{array}{llll}0.9000255779573720 & 0.6069591255080411 & 0.1341229497090097\end{array}$ 
$\begin{array}{llll}0.8001847946659950 & 0.4398080694128836 & 0.1339687646835301\end{array}$

$\begin{array}{llll}0.9000154399023401 & 0.9384560642742059 & 0.1339354265835695\end{array}$

$\begin{array}{llll}0.8002600746148900 & 0.7732888300066492 & 0.1340279172821539\end{array}$

$\begin{array}{lllll}0.0000000000000000 & 0.2222209999999976 & 0.0666289999999989\end{array}$

$\begin{array}{lllll}0.1000039999999984 & 0.0555549999999982 & 0.0666289999999989\end{array}$

$\begin{array}{lllll}0.3000139999999973 & 0.0555549999999982 & 0.0666289999999989\end{array}$

$\begin{array}{llll}0.2000090000000014 & 0.2222209999999976 & 0.0666289999999989\end{array}$

$\begin{array}{lllll}0.0000000000000000 & 0.5555540000000008 & 0.0666289999999989\end{array}$

$\begin{array}{lllll}0.1000039999999984 & 0.3888880000000015 & 0.0666289999999989\end{array}$

$\begin{array}{llll}0.3000139999999973 & 0.3888880000000015 & 0.0666289999999989\end{array}$

$\begin{array}{llll}0.2000090000000014 & 0.5555540000000008 & 0.0666289999999989\end{array}$

$\begin{array}{llll}0.0000000000000000 & 0.8888869999999969 & 0.0666289999999989\end{array}$

$\begin{array}{lllll}0.1000039999999984 & 0.7222220000000021 & 0.0666289999999989\end{array}$

$\begin{array}{lllll}0.3000139999999973 & 0.7222220000000021 & 0.0666289999999989\end{array}$

$\begin{array}{lllll}0.2000090000000014 & 0.8888869999999969 & 0.0666289999999989\end{array}$

$\begin{array}{llll}0.3999999999999986 & 0.2222209999999976 & 0.0666289999999989\end{array}$

$\begin{array}{lllll}0.5000039999999970 & 0.0555549999999982 & 0.0666289999999989\end{array}$

$\begin{array}{lllll}0.7000140000000030 & 0.0555549999999982 & 0.0666289999999989\end{array}$

$\begin{array}{lllll}0.6000090000000000 & 0.2222209999999976 & 0.0666289999999989\end{array}$

$\begin{array}{llll}0.3999999999999986 & 0.5555540000000008 & 0.0666289999999989\end{array}$

$\begin{array}{llll}0.5000039999999970 & 0.3888880000000015 & 0.0666289999999989\end{array}$

$\begin{array}{llll}0.7000140000000030 & 0.3888880000000015 & 0.0666289999999989\end{array}$

$\begin{array}{lllll}0.6000090000000000 & 0.5555540000000008 & 0.0666289999999989\end{array}$

$\begin{array}{llll}0.3999999999999986 & 0.8888869999999969 & 0.0666289999999989\end{array}$

$\begin{array}{lllll}0.5000039999999970 & 0.7222220000000021 & 0.0666289999999989\end{array}$

$\begin{array}{lllll}0.7000140000000030 & 0.7222220000000021 & 0.0666289999999989\end{array}$

$\begin{array}{lllll}0.6000090000000000 & 0.8888869999999969 & 0.0666289999999989\end{array}$

$\begin{array}{lllll}0.7999999999999972 & 0.2222209999999976 & 0.0666289999999989\end{array}$

$\begin{array}{llll}0.9000040000000027 & 0.0555549999999982 & 0.0666289999999989\end{array}$

$\begin{array}{llll}0.7999999999999972 & 0.5555540000000008 & 0.0666289999999989\end{array}$

$\begin{array}{llll}0.9000040000000027 & 0.3888880000000015 & 0.0666289999999989\end{array}$

$\begin{array}{llll}0.7999999999999972 & 0.8888869999999969 & 0.0666289999999989\end{array}$

$\begin{array}{lllll}0.9000040000000027 & 0.7222220000000021 & 0.0666289999999989\end{array}$

$0.10000399999999840 .1666650000000018 \quad 0.0000000000000000$

$\begin{array}{llll}0.0000000000000000 & 0.0000000000000000 & 0.0000000000000000\end{array}$

$0.3000139999999973 \quad 0.16666500000000180 .0000000000000000$

$0.2000090000000014 \quad 0.0000000000000000 \quad 0.0000000000000000$

$\begin{array}{lll}0.1000039999999984 & 0.4999990000000025 & 0.0000000000000000\end{array}$

$\begin{array}{llll}0.0000000000000000 & 0.3333330000000032 & 0.0000000000000000\end{array}$

$\begin{array}{llll}0.3000139999999973 & 0.4999990000000025 & 0.0000000000000000\end{array}$

$\begin{array}{llll}0.2000090000000014 & 0.3333330000000032 & 0.0000000000000000\end{array}$

$\begin{array}{llll}0.1000039999999984 & 0.8333319999999986 & 0.0000000000000000\end{array}$

$\begin{array}{llll}0.0000000000000000 & 0.6666669999999968 & 0.0000000000000000\end{array}$

$\begin{array}{llll}0.3000139999999973 & 0.8333319999999986 & 0.0000000000000000\end{array}$

$\begin{array}{llll}0.2000090000000014 & 0.6666669999999968 & 0.0000000000000000\end{array}$

$\begin{array}{llll}0.5000039999999970 & 0.1666650000000018 & 0.0000000000000000\end{array}$

$\begin{array}{lllll}0.3999999999999986 & 0.0000000000000000 & 0.0000000000000000\end{array}$ 
$\begin{array}{llll}0.7000140000000030 & 0.1666650000000018 & 0.0000000000000000\end{array}$

$\begin{array}{lllll}0.6000090000000000 & 0.0000000000000000 & 0.0000000000000000\end{array}$

$\begin{array}{lllll}0.5000039999999970 & 0.4999990000000025 & 0.0000000000000000\end{array}$

$\begin{array}{lllll}0.3999999999999986 & 0.33333330000000032 & 0.0000000000000000\end{array}$

$\begin{array}{llll}0.7000140000000030 & 0.4999990000000025 & 0.0000000000000000\end{array}$

$\begin{array}{lllll}0.6000090000000000 & 0.3333330000000032 & 0.0000000000000000\end{array}$

$\begin{array}{lllll}0.5000039999999970 & 0.83333319999999986 & 0.0000000000000000\end{array}$

$\begin{array}{lllll}0.3999999999999986 & 0.6666669999999968 & 0.0000000000000000\end{array}$

$\begin{array}{llll}0.7000140000000030 & 0.8333319999999986 & 0.0000000000000000\end{array}$

$\begin{array}{lllll}0.6000090000000000 & 0.6666669999999968 & 0.0000000000000000\end{array}$

$\begin{array}{lllll}0.9000040000000027 & 0.1666650000000018 & 0.0000000000000000\end{array}$

$\begin{array}{lllll}0.7999999999999972 & 0.0000000000000000 & 0.0000000000000000\end{array}$

$\begin{array}{lllll}0.9000040000000027 & 0.4999990000000025 & 0.0000000000000000\end{array}$

$\begin{array}{lllll}0.7999999999999972 & 0.33333330000000032 & 0.0000000000000000\end{array}$

$\begin{array}{lllll}0.9000040000000027 & 0.83333319999999986 & 0.0000000000000000\end{array}$

$\begin{array}{lllll}0.7999999999999972 & 0.6666669999999968 & 0.0000000000000000\end{array}$

$\begin{array}{lllll}0.3979102917305113 & 0.5777952247161480 & 0.2166418883284749\end{array}$

$\begin{array}{lllll}0.5383825156441802 & 0.5746015396781061 & 0.2232063508959925\end{array}$

$\begin{array}{lllll}0.3963192849348260 & 0.4374652089819335 & 0.2099865010032076\end{array}$

$\begin{array}{lllll}0.2562366839879360 & 0.5772293683332776 & 0.2210727266045822\end{array}$

$\begin{array}{llllll}0.3991962030320053 & 0.7120611328179365 & 0.2270941233832602\end{array}$

$\begin{array}{lllll}0.6928027099326073 & 0.5258311770796809 & 0.2270011454954073\end{array}$

$\begin{array}{llllll}0.1021719088692663 & 0.6227734967257490 & 0.2306381486595868\end{array}$

$\begin{array}{lllll}0.3523233318859980 & 0.8610196099956156 & 0.2352532371188616\end{array}$

$\begin{array}{lllll}0.4484207805845628 & 0.8607988004189338 & 0.2337245477389374\end{array}$

$\begin{array}{lllll}0.6944693493728877 & 0.6183112666065398 & 0.2290369266653869\end{array}$

$\begin{array}{lllll}0.1009245732485269 & 0.5315165881143287 & 0.2236745352607485\end{array}$

$\begin{array}{lllll}0.4487941502990651 & 0.2887037437492808 & 0.1971262422967399\end{array}$

$\begin{array}{lllll}0.3437276896550543 & 0.2902213346159002 & 0.1951884895148694\end{array}$

$\begin{array}{lllll}0.5668349921008513 & 0.4106949682135621 & 0.2145096072294024\end{array}$

$\begin{array}{lllll}0.2290566736255357 & 0.7398687132688622 & 0.2322140759731113\end{array}$

$\begin{array}{lllll}0.5957711110483217 & 0.4998237299191288 & 0.2225285954876739\end{array}$

$\begin{array}{lllll}0.3223510535987657 & 0.7686090559683123 & 0.2312117765445905\end{array}$

$\begin{array}{lllll}0.5984394726065716 & 0.6485913503220715 & 0.2270096494425147\end{array}$

$\begin{array}{llllll}0.1984591905973119 & 0.6512872402803254 & 0.2288551773986001\end{array}$

$\begin{array}{lllll}0.1968425332164401 & 0.5051122204512239 & 0.2169116280877077\end{array}$

$\begin{array}{lllll}0.4750609120395808 & 0.3812439874361999 & 0.2086077827378290\end{array}$

$\begin{array}{lllll}0.4768654330320956 & 0.7681436216308057 & 0.2289270603436009\end{array}$

$\begin{array}{lllll}0.3188746983356125 & 0.3852056068524519 & 0.2034794304520275\end{array}$

$\begin{array}{lllll}0.5701071618848346 & 0.7382276063580022 & 0.2281448286302634\end{array}$

$\begin{array}{lllll}0.2236331350476632 & 0.4178766712432136 & 0.2028503073845016\end{array}$

$\begin{array}{lllll}0.7513308767827880 & 0.4792935509702403 & 0.2272228063519748\end{array}$

$\begin{array}{llllll}0.0432149708613068 & 0.6670556182749294 & 0.2359372107896931\end{array}$

$\begin{array}{lllll}0.0411547718145684 & 0.4866072804158250 & 0.2221875699916041\end{array}$

$\begin{array}{lllll}0.7546533184984909 & 0.6623989065765343 & 0.2316879024185710\end{array}$

$\begin{array}{lllll}0.4960299588600421 & 0.9175311467628120 & 0.2356422970136762\end{array}$

$\begin{array}{lllll}0.3055323809978887 & 0.9179703493692983 & 0.2386884588873244\end{array}$ 
$\begin{array}{llll}0.4832728840779907 & 0.2322227902900238 & 0.2113036209940375\end{array}$

$\begin{array}{llll}0.3040990859527071 & 0.2371910759272211 & 0.2096513011265770\end{array}$

$\begin{array}{llll}0.1756703136097855 & 0.7914001643668342 & 0.2361213861712912\end{array}$

$\begin{array}{llll}0.6210534477389018 & 0.3592416963372713 & 0.2143610307632855\end{array}$

$\begin{array}{llll}0.6246385419306151 & 0.7891867415530385 & 0.2301729916512940\end{array}$

$\begin{array}{lllll}0.1713255437921006 & 0.3651306693372121 & 0.2069034769268683\end{array}$

\section{FeP-Pt(111) - Hfcc-0, vdW-DF-revPBE:}

$\mathrm{Pt} \mathrm{Fe} \mathrm{N} \mathrm{C} \mathrm{H}$

1.00000000000000

$\begin{array}{lll}14.2799999999999994 & 0.0000000000000000 & 0.0000000000000000\end{array}$

$\begin{array}{llll}0.0000000000000000 & 14.8409999999999993 & 0.0000000000000000\end{array}$

$\begin{array}{llll}0.0000000000000000 & 0.0000000000000000 & 35.0000000000000000\end{array}$

$\mathrm{Pt} F \mathrm{Ne} \mathrm{C} \mathrm{H}$

$\begin{array}{lllll}90 & 1 & 4 & 20 & 12\end{array}$

Direct

$\begin{array}{llll}0.7999999999999972 & 0.6666669999999968 & 0.0000000000000000\end{array}$

$\begin{array}{llll}0.9000040000000027 & 0.8333319999999986 & 0.0000000000000000\end{array}$

$\begin{array}{llll}0.7999999999999972 & 0.3333330000000032 & 0.0000000000000000\end{array}$

$\begin{array}{llll}0.9000040000000027 & 0.4999990000000025 & 0.0000000000000000\end{array}$

$\begin{array}{llll}0.7999999999999972 & 0.0000000000000000 & 0.0000000000000000\end{array}$

$\begin{array}{llll}0.9000040000000027 & 0.1666650000000018 & 0.0000000000000000\end{array}$

$\begin{array}{llll}0.6000090000000000 & 0.6666669999999968 & 0.0000000000000000\end{array}$

$\begin{array}{llll}0.7000140000000030 & 0.8333319999999986 & 0.0000000000000000\end{array}$

$\begin{array}{llll}0.3999999999999986 & 0.6666669999999968 & 0.0000000000000000\end{array}$

$\begin{array}{llll}0.5000039999999970 & 0.8333319999999986 & 0.0000000000000000\end{array}$

$\begin{array}{llll}0.6000090000000000 & 0.3333330000000032 & 0.0000000000000000\end{array}$

$\begin{array}{llll}0.7000140000000030 & 0.4999990000000025 & 0.0000000000000000\end{array}$

$\begin{array}{lllll}0.3999999999999986 & 0.3333330000000032 & 0.0000000000000000\end{array}$

$\begin{array}{llll}0.5000039999999970 & 0.4999990000000025 & 0.0000000000000000\end{array}$

$\begin{array}{llll}0.6000090000000000 & 0.0000000000000000 & 0.0000000000000000\end{array}$

$\begin{array}{llll}0.7000140000000030 & 0.1666650000000018 & 0.0000000000000000\end{array}$

$\begin{array}{llll}0.3999999999999986 & 0.0000000000000000 & 0.0000000000000000\end{array}$

$\begin{array}{llll}0.5000039999999970 & 0.1666650000000018 & 0.0000000000000000\end{array}$

$\begin{array}{lllll}0.2000090000000014 & 0.6666669999999968 & 0.0000000000000000\end{array}$

$\begin{array}{lllll}0.3000139999999973 & 0.8333319999999986 & 0.0000000000000000\end{array}$

$\begin{array}{lllll}0.0000000000000000 & 0.6666669999999968 & 0.0000000000000000\end{array}$

0.10000399999999840 .83333199999999860 .0000000000000000

$\begin{array}{llll}0.2000090000000014 & 0.3333330000000032 & 0.0000000000000000\end{array}$

$\begin{array}{llll}0.3000139999999973 & 0.4999990000000025 & 0.0000000000000000\end{array}$

$\begin{array}{lllll}0.0000000000000000 & 0.3333330000000032 & 0.0000000000000000\end{array}$

$\begin{array}{lllll}0.1000039999999984 & 0.4999990000000025 & 0.0000000000000000\end{array}$

$0.20000900000000140 .0000000000000000 \quad 0.0000000000000000$

$\begin{array}{llll}0.3000139999999973 & 0.1666650000000018 & 0.0000000000000000\end{array}$

$\begin{array}{llll}0.0000000000000000 & 0.0000000000000000 & 0.0000000000000000\end{array}$

$\begin{array}{lllll}0.1000039999999984 & 0.1666650000000018 & 0.0000000000000000\end{array}$

$\begin{array}{llll}0.9000040000000027 & 0.7222220000000021 & 0.0666289999999989\end{array}$ 
$\begin{array}{llll}0.7999999999999972 & 0.8888869999999969 & 0.0666289999999989\end{array}$

$\begin{array}{lllll}0.9000040000000027 & 0.3888880000000015 & 0.0666289999999989\end{array}$

$\begin{array}{llll}0.7999999999999972 & 0.5555540000000008 & 0.0666289999999989\end{array}$

$\begin{array}{lllll}0.9000040000000027 & 0.0555549999999982 & 0.0666289999999989\end{array}$

$\begin{array}{lllll}0.7999999999999972 & 0.2222209999999976 & 0.0666289999999989\end{array}$

$\begin{array}{llll}0.6000090000000000 & 0.8888869999999969 & 0.0666289999999989\end{array}$

$\begin{array}{llll}0.7000140000000030 & 0.7222220000000021 & 0.0666289999999989\end{array}$

$\begin{array}{lllll}0.5000039999999970 & 0.7222220000000021 & 0.0666289999999989\end{array}$

$\begin{array}{llll}0.3999999999999986 & 0.8888869999999969 & 0.0666289999999989\end{array}$

$\begin{array}{llll}0.6000090000000000 & 0.5555540000000008 & 0.0666289999999989\end{array}$

$\begin{array}{llll}0.7000140000000030 & 0.3888880000000015 & 0.0666289999999989\end{array}$

$\begin{array}{llll}0.5000039999999970 & 0.3888880000000015 & 0.0666289999999989\end{array}$

$\begin{array}{lllll}0.3999999999999986 & 0.5555540000000008 & 0.0666289999999989\end{array}$

$\begin{array}{llll}0.6000090000000000 & 0.2222209999999976 & 0.0666289999999989\end{array}$

$\begin{array}{llll}0.7000140000000030 & 0.0555549999999982 & 0.0666289999999989\end{array}$

$\begin{array}{lllll}0.5000039999999970 & 0.0555549999999982 & 0.0666289999999989\end{array}$

$\begin{array}{lllll}0.3999999999999986 & 0.2222209999999976 & 0.0666289999999989\end{array}$

$\begin{array}{lllll}0.2000090000000014 & 0.8888869999999969 & 0.0666289999999989\end{array}$

$\begin{array}{llll}0.3000139999999973 & 0.7222220000000021 & 0.0666289999999989\end{array}$

$\begin{array}{lllll}0.1000039999999984 & 0.7222220000000021 & 0.0666289999999989\end{array}$

$\begin{array}{lllll}0.0000000000000000 & 0.8888869999999969 & 0.0666289999999989\end{array}$

$\begin{array}{lllll}0.2000090000000014 & 0.5555540000000008 & 0.0666289999999989\end{array}$

$\begin{array}{lllll}0.3000139999999973 & 0.3888880000000015 & 0.0666289999999989\end{array}$

$\begin{array}{llll}0.1000039999999984 & 0.3888880000000015 & 0.0666289999999989\end{array}$

$\begin{array}{lllll}0.0000000000000000 & 0.5555540000000008 & 0.0666289999999989\end{array}$

$\begin{array}{lllll}0.2000090000000014 & 0.2222209999999976 & 0.0666289999999989\end{array}$

$\begin{array}{lllll}0.3000139999999973 & 0.0555549999999982 & 0.0666289999999989\end{array}$

$\begin{array}{lllll}0.1000039999999984 & 0.0555549999999982 & 0.0666289999999989\end{array}$

$\begin{array}{lllll}0.0000000000000000 & 0.2222209999999976 & 0.0666289999999989\end{array}$

$\begin{array}{llll}0.8005131760001737 & 0.7769012676910909 & 0.1340578993612982\end{array}$

$\begin{array}{lllll}0.9002711638844804 & 0.9422397821009616 & 0.1341720719811581\end{array}$

$\begin{array}{llll}0.8016697542708774 & 0.4443678875097842 & 0.1329052775422494\end{array}$

$\begin{array}{llll}0.9017037013849967 & 0.6112107281946192 & 0.1342756002901531\end{array}$

$\begin{array}{llll}0.8009272333125307 & 0.1074065885207695 & 0.1338458592008376\end{array}$

$\begin{array}{llll}0.9006930252687002 & 0.2760808195513830 & 0.1333574644890680\end{array}$

$\begin{array}{lllll}0.4004343697197189 & 0.7758238020767706 & 0.1338729234990455\end{array}$

$\begin{array}{lllll}0.5997929157199973 & 0.7757825732464266 & 0.1337887978747584\end{array}$

$\begin{array}{llll}0.7004208945637072 & 0.9411833104298939 & 0.1342592665511733\end{array}$

$\begin{array}{llll}0.5000526816542243 & 0.9405104404011787 & 0.1344053518010224\end{array}$

$\begin{array}{llll}0.3989652476441065 & 0.4480334622185810 & 0.1319944600141220\end{array}$

$\begin{array}{lllll}0.6006408547794204 & 0.4478340086662722 & 0.1317475596688474\end{array}$

$\begin{array}{lllll}0.7028224520708067 & 0.6115893204773687 & 0.1332023017570974\end{array}$

$\begin{array}{llll}0.4999537454544253 & 0.6105362131663463 & 0.1358475618702150\end{array}$

$\begin{array}{llll}0.3994036457561076 & 0.1044366198835835 & 0.1329535444529906\end{array}$

$\begin{array}{llll}0.6004881313525016 & 0.1047923047267897 & 0.1329693230674106\end{array}$

$\begin{array}{lllll}0.7005793333728645 & 0.2737123236931971 & 0.1374686625351136\end{array}$

$\begin{array}{llll}0.4994099797520163 & 0.2739059441647184 & 0.1345507154525248\end{array}$ 
$\begin{array}{llll}0.9999476444104665 & 0.7772610178622517 & 0.1343808168424449\end{array}$

$\begin{array}{llll}0.1994526014772693 & 0.7769514737732207 & 0.1340401645445244\end{array}$

$\begin{array}{llll}0.2995185191523140 & 0.9410610404274290 & 0.1342558161317582\end{array}$

$\begin{array}{llll}0.0995238387088219 & 0.9423065879494179 & 0.1342200010040054\end{array}$

$\begin{array}{llll}0.9998938583238958 & 0.4443813107966083 & 0.1344526515659509\end{array}$

$\begin{array}{llll}0.1981339898293662 & 0.4444489173297529 & 0.1328574555664655\end{array}$

$\begin{array}{lllll}0.2972269064082816 & 0.6117413331013037 & 0.1331690639839171\end{array}$

$\begin{array}{llll}0.0982768906488724 & 0.6112741830918562 & 0.1342541852867255\end{array}$

$\begin{array}{llll}0.9998274488762888 & 0.1089720244317238 & 0.1340133538102992\end{array}$

$\begin{array}{llll}0.1987578596915822 & 0.1071764003739593 & 0.1338027244703222\end{array}$

$\begin{array}{llll}0.2987425050923141 & 0.2733580175770697 & 0.1373176642088158\end{array}$

$\begin{array}{llll}0.0986802214804332 & 0.2761844240447789 & 0.1332618239812838\end{array}$

$\begin{array}{lllll}0.4967682183065527 & 0.5153054269512797 & 0.2072189151820074\end{array}$

$\begin{array}{llll}0.5972061517725573 & 0.6060681566598390 & 0.2270191070593395\end{array}$

$\begin{array}{llll}0.3951450314831936 & 0.6047849975646395 & 0.2270566861753186\end{array}$

$\begin{array}{llll}0.3948767783695478 & 0.4086102933341243 & 0.2135596437149044\end{array}$

$\begin{array}{lllll}0.5997706494561627 & 0.4098614134029464 & 0.2135772217951320\end{array}$

$\begin{array}{llll}0.2553589989882554 & 0.5058561512605182 & 0.2254405995401328\end{array}$

$\begin{array}{llll}0.7381000627617088 & 0.5086507573892580 & 0.2257597153527971\end{array}$

$\begin{array}{llll}0.2974363770807398 & 0.4212811008292904 & 0.2189245408673310\end{array}$

$\begin{array}{llll}0.6934785341584796 & 0.5924609561738912 & 0.2278816568032558\end{array}$

$\begin{array}{lllll}0.4082979394721187 & 0.3199927320719544 & 0.2056998571500920\end{array}$

$\begin{array}{llll}0.2990478497259489 & 0.5900319330733539 & 0.2278271643976790\end{array}$

$\begin{array}{llll}0.4077806064830430 & 0.6969228253146195 & 0.2301250708250871\end{array}$

$\begin{array}{llll}0.6969732534704339 & 0.4238121537818504 & 0.2191529309215395\end{array}$

$\begin{array}{llll}0.5832549530356846 & 0.6981639586635385 & 0.2300075018754881\end{array}$

$\begin{array}{lllll}0.5875837363438589 & 0.3210773319423817 & 0.2056940562590734\end{array}$

$\begin{array}{llll}0.4952499527799695 & 0.7402016114007122 & 0.2312482282710338\end{array}$

$\begin{array}{llll}0.4983365060683621 & 0.2776546769142262 & 0.1982384602981530\end{array}$

$\begin{array}{llll}0.2498043334404334 & 0.3380267259162354 & 0.2139292513822181\end{array}$

$\begin{array}{lllll}0.3176921222586899 & 0.2724549747531489 & 0.2031579764301609\end{array}$

$\begin{array}{lllll}0.2503231953007417 & 0.6758536561565169 & 0.2312022977713441\end{array}$

$\begin{array}{llll}0.7457415453415126 & 0.3411465768833123 & 0.2142279733600479\end{array}$

$\begin{array}{llll}0.7409805440507095 & 0.6788312260979114 & 0.2312911069158512\end{array}$

$\begin{array}{llll}0.6730651793496181 & 0.7439697372421475 & 0.2323189085184425\end{array}$

$\begin{array}{llll}0.3174604877801535 & 0.7417499400339370 & 0.2323696780055568\end{array}$

$\begin{array}{llll}0.6789876041873067 & 0.2745123515177142 & 0.2032455571618903\end{array}$

$\begin{array}{llll}0.1792498730759986 & 0.5059576742650834 & 0.2271946334383960\end{array}$

$\begin{array}{llll}0.8142094646870345 & 0.5094425206498198 & 0.2276742952237977\end{array}$

$\begin{array}{llll}0.4988210687453694 & 0.2049665194978878 & 0.2033044046114093\end{array}$

$\begin{array}{llll}0.4948126986786401 & 0.8133930991113647 & 0.2330862394379495\end{array}$

$\begin{array}{llll}0.1755217002634103 & 0.3268149603710934 & 0.2180450560966493\end{array}$

$\begin{array}{llll}0.3105684005788807 & 0.2003906245657591 & 0.2079505901744965\end{array}$

$\begin{array}{llll}0.6822805837633802 & 0.8163856825062806 & 0.2339346808295454\end{array}$

$\begin{array}{llll}0.8163564395567260 & 0.6875857850623018 & 0.2319458075203527\end{array}$

$\begin{array}{llll}0.8201100704992026 & 0.3308707652701682 & 0.2184886446064559\end{array}$

$\begin{array}{lllll}0.1748603242677862 & 0.6838297801779577 & 0.2314398753798834\end{array}$ 
$\begin{array}{llll}0.3074916874878094 & 0.8140742539798896 & 0.2337434305501661\end{array}$

$\begin{array}{llll}0.6869378235407896 & 0.2025786062343918 & 0.2082393658600855\end{array}$

\section{FeP-Pt(111) - Hfcc-45, vdW-DF-revPBE:}

Pt Fe N C H

1.00000000000000

$\begin{array}{lll}14.2799999999999994 & 0.0000000000000000 & 0.0000000000000000\end{array}$

$\begin{array}{lll}0.0000000000000000 & 14.8409999999999993 & 0.0000000000000000\end{array}$

$\begin{array}{llll}0.0000000000000000 & 0.0000000000000000 & 35.0000000000000000\end{array}$

$\mathrm{Pt} \mathrm{Fe} \mathrm{N}$ C H

$\begin{array}{lllll}90 & 1 & 4 & 20 & 12\end{array}$

Direct

$\begin{array}{llll}0.7999999999999972 & 0.6666669999999968 & 0.0000000000000000\end{array}$

$\begin{array}{llll}0.9000040000000027 & 0.8333319999999986 & 0.0000000000000000\end{array}$

$\begin{array}{llll}0.7999999999999972 & 0.3333330000000032 & 0.0000000000000000\end{array}$

$\begin{array}{llll}0.9000040000000027 & 0.4999990000000025 & 0.0000000000000000\end{array}$

$\begin{array}{llll}0.7999999999999972 & 0.0000000000000000 & 0.0000000000000000\end{array}$

$\begin{array}{llll}0.9000040000000027 & 0.1666650000000018 & 0.0000000000000000\end{array}$

$\begin{array}{llll}0.6000090000000000 & 0.6666669999999968 & 0.0000000000000000\end{array}$

$\begin{array}{llll}0.7000140000000030 & 0.8333319999999986 & 0.0000000000000000\end{array}$

$\begin{array}{llll}0.3999999999999986 & 0.6666669999999968 & 0.0000000000000000\end{array}$

$\begin{array}{llll}0.5000039999999970 & 0.8333319999999986 & 0.0000000000000000\end{array}$

$\begin{array}{llll}0.6000090000000000 & 0.3333330000000032 & 0.0000000000000000\end{array}$

$\begin{array}{lll}0.7000140000000030 & 0.4999990000000025 & 0.0000000000000000\end{array}$

$\begin{array}{llll}0.3999999999999986 & 0.3333330000000032 & 0.0000000000000000\end{array}$

$\begin{array}{llll}0.5000039999999970 & 0.4999990000000025 & 0.0000000000000000\end{array}$

$\begin{array}{llll}0.6000090000000000 & 0.0000000000000000 & 0.0000000000000000\end{array}$

$\begin{array}{llll}0.7000140000000030 & 0.1666650000000018 & 0.0000000000000000\end{array}$

$\begin{array}{llll}0.3999999999999986 & 0.0000000000000000 & 0.0000000000000000\end{array}$

$\begin{array}{llll}0.5000039999999970 & 0.1666650000000018 & 0.0000000000000000\end{array}$

$\begin{array}{llll}0.2000090000000014 & 0.6666669999999968 & 0.0000000000000000\end{array}$

$\begin{array}{llll}0.3000139999999973 & 0.8333319999999986 & 0.0000000000000000\end{array}$

$\begin{array}{llll}0.0000000000000000 & 0.6666669999999968 & 0.0000000000000000\end{array}$

$\begin{array}{llll}0.1000039999999984 & 0.8333319999999986 & 0.0000000000000000\end{array}$

$\begin{array}{llll}0.2000090000000014 & 0.3333330000000032 & 0.0000000000000000\end{array}$

$\begin{array}{llll}0.3000139999999973 & 0.4999990000000025 & 0.0000000000000000\end{array}$

$\begin{array}{llll}0.0000000000000000 & 0.3333330000000032 & 0.0000000000000000\end{array}$

0.10000399999999840 .49999900000000250 .0000000000000000

$\begin{array}{llll}0.2000090000000014 & 0.0000000000000000 & 0.0000000000000000\end{array}$

$\begin{array}{llll}0.3000139999999973 & 0.1666650000000018 & 0.0000000000000000\end{array}$

$\begin{array}{llll}0.0000000000000000 & 0.0000000000000000 & 0.0000000000000000\end{array}$

$\begin{array}{llll}0.1000039999999984 & 0.1666650000000018 & 0.0000000000000000\end{array}$

$\begin{array}{llll}0.9000040000000027 & 0.7222220000000021 & 0.0666289999999989\end{array}$

$\begin{array}{llll}0.7999999999999972 & 0.8888869999999969 & 0.0666289999999989\end{array}$

$\begin{array}{lllll}0.9000040000000027 & 0.3888880000000015 & 0.0666289999999989\end{array}$

$\begin{array}{llll}0.7999999999999972 & 0.5555540000000008 & 0.0666289999999989\end{array}$

$\begin{array}{llll}0.9000040000000027 & 0.0555549999999982 & 0.0666289999999989\end{array}$ 
$\begin{array}{llll}0.7999999999999972 & 0.2222209999999976 & 0.0666289999999989\end{array}$

$\begin{array}{llll}0.6000090000000000 & 0.8888869999999969 & 0.0666289999999989\end{array}$

$\begin{array}{lllll}0.7000140000000030 & 0.7222220000000021 & 0.0666289999999989\end{array}$

$\begin{array}{lllll}0.5000039999999970 & 0.7222220000000021 & 0.0666289999999989\end{array}$

$\begin{array}{llll}0.3999999999999986 & 0.8888869999999969 & 0.0666289999999989\end{array}$

$\begin{array}{llll}0.6000090000000000 & 0.5555540000000008 & 0.0666289999999989\end{array}$

$\begin{array}{llll}0.7000140000000030 & 0.3888880000000015 & 0.0666289999999989\end{array}$

$\begin{array}{llll}0.5000039999999970 & 0.3888880000000015 & 0.0666289999999989\end{array}$

$\begin{array}{llll}0.3999999999999986 & 0.5555540000000008 & 0.0666289999999989\end{array}$

$\begin{array}{llll}0.6000090000000000 & 0.2222209999999976 & 0.0666289999999989\end{array}$

$\begin{array}{llll}0.7000140000000030 & 0.0555549999999982 & 0.0666289999999989\end{array}$

$\begin{array}{lllll}0.5000039999999970 & 0.0555549999999982 & 0.0666289999999989\end{array}$

$\begin{array}{lllll}0.3999999999999986 & 0.2222209999999976 & 0.0666289999999989\end{array}$

$\begin{array}{llll}0.2000090000000014 & 0.8888869999999969 & 0.0666289999999989\end{array}$

$\begin{array}{llll}0.3000139999999973 & 0.7222220000000021 & 0.0666289999999989\end{array}$

$\begin{array}{lllll}0.1000039999999984 & 0.7222220000000021 & 0.0666289999999989\end{array}$

$\begin{array}{lllll}0.0000000000000000 & 0.8888869999999969 & 0.0666289999999989\end{array}$

$\begin{array}{llll}0.2000090000000014 & 0.5555540000000008 & 0.0666289999999989\end{array}$

$\begin{array}{llll}0.3000139999999973 & 0.3888880000000015 & 0.0666289999999989\end{array}$

$\begin{array}{llll}0.1000039999999984 & 0.3888880000000015 & 0.0666289999999989\end{array}$

$\begin{array}{lllll}0.0000000000000000 & 0.5555540000000008 & 0.0666289999999989\end{array}$

$\begin{array}{lllll}0.2000090000000014 & 0.2222209999999976 & 0.0666289999999989\end{array}$

$\begin{array}{llll}0.3000139999999973 & 0.0555549999999982 & 0.0666289999999989\end{array}$

$\begin{array}{lllll}0.1000039999999984 & 0.0555549999999982 & 0.0666289999999989\end{array}$

$\begin{array}{lllll}0.0000000000000000 & 0.2222209999999976 & 0.0666289999999989\end{array}$

$\begin{array}{llll}0.8023620515331298 & 0.7829005977286868 & 0.1331014845294475\end{array}$

$\begin{array}{llll}0.9010694618275554 & 0.9476279954070417 & 0.1344448644765082\end{array}$

$\begin{array}{llll}0.8014609764189642 & 0.4460318083867386 & 0.1328317860363775\end{array}$

$\begin{array}{llll}0.9003575038962698 & 0.6137030330994784 & 0.1332765772635243\end{array}$

$\begin{array}{llll}0.8013111812489041 & 0.1134292023332357 & 0.1345898813022611\end{array}$

$\begin{array}{llll}0.9005976121042506 & 0.2788710620823333 & 0.1343200672400810\end{array}$

$\begin{array}{llll}0.3998907271286706 & 0.7867658940740415 & 0.1357357064582287\end{array}$

$\begin{array}{llll}0.5977804024733473 & 0.7858453946214196 & 0.1356393744994440\end{array}$

$\begin{array}{llll}0.7021480096785130 & 0.9493770137090172 & 0.1340910156604688\end{array}$

$\begin{array}{llll}0.4997316641898433 & 0.9516252875709975 & 0.1333622772136832\end{array}$

$\begin{array}{llll}0.3995198568084486 & 0.4398541157977974 & 0.1335900019623526\end{array}$

$\begin{array}{llll}0.6003747810193147 & 0.4398470568291348 & 0.1336511149153383\end{array}$

$\begin{array}{llll}0.7005565936458162 & 0.6179389297659134 & 0.1376927530847879\end{array}$

$\begin{array}{llll}0.4998143245259773 & 0.6024990684005809 & 0.1310873853312865\end{array}$

$\begin{array}{llll}0.3986058355742637 & 0.1130946198653852 & 0.1345955350063844\end{array}$

$\begin{array}{lllll}0.6013723883545601 & 0.1130871539977806 & 0.1345938738971603\end{array}$

$\begin{array}{llll}0.7014578786771026 & 0.2782975404066358 & 0.1341679013687165\end{array}$

$\begin{array}{llll}0.5000975304170531 & 0.2752450404928086 & 0.1331558291783648\end{array}$

$\begin{array}{llll}0.9996380472817795 & 0.7807886167109033 & 0.1345260929442162\end{array}$

$\begin{array}{lllll}0.1964070231575513 & 0.7834159029691334 & 0.1333328344445765\end{array}$

$\begin{array}{llll}0.2968439571726929 & 0.9499316522659962 & 0.1341970691619762\end{array}$

$\begin{array}{lllll}0.0983268164648203 & 0.9478127781882967 & 0.1344902500056611\end{array}$ 
$\begin{array}{llll}0.9999751493131024 & 0.4462023838919030 & 0.1339442174304385\end{array}$ $\begin{array}{llll}0.1985123241411926 & 0.4460784467434152 & 0.1328177296902935\end{array}$ $\begin{array}{lllll}0.2985953026163484 & 0.6186449358880171 & 0.1376754001429091\end{array}$ $\begin{array}{llll}0.0988077734062146 & 0.6139052123277730 & 0.1333389077451737\end{array}$ $\begin{array}{llll}0.9999411796844138 & 0.1134294289241014 & 0.1343891391596212\end{array}$ $\begin{array}{llll}0.1984835892072785 & 0.1135427921042579 & 0.1346534904449226\end{array}$ $\begin{array}{llll}0.2985603715368817 & 0.2783123168411710 & 0.1341659263550440\end{array}$ $\begin{array}{llll}0.0993878498447129 & 0.2789020578036320 & 0.1342651144729695\end{array}$ $\begin{array}{llll}0.4986375948229483 & 0.4720883617567602 & 0.2019807574838085\end{array}$ $\begin{array}{llll}0.4984461251451704 & 0.6218796011512445 & 0.2005942469904924\end{array}$ $\begin{array}{llll}0.3545747425437714 & 0.4800584622618180 & 0.2190202711081522\end{array}$ $\begin{array}{lllll}0.4985110211164901 & 0.3414967539192334 & 0.2267527397830480\end{array}$ $\begin{array}{llll}0.6423750708828534 & 0.4808863210418634 & 0.2191102346996502\end{array}$ $\begin{array}{llll}0.3274601072089187 & 0.3167116008870389 & 0.2288510403914590\end{array}$ $\begin{array}{llll}0.6724770553542391 & 0.6422887144358316 & 0.2017900189581881\end{array}$ $\begin{array}{lllll}0.4211674138020829 & 0.2859591094083199 & 0.2293678617833599\end{array}$ $\begin{array}{llll}0.5774571089484060 & 0.6768954742600880 & 0.2003098973705136\end{array}$ $\begin{array}{llll}0.5760974259427818 & 0.2864583333424472 & 0.2298894113242569\end{array}$ $\begin{array}{lllll}0.2964538693583939 & 0.4057784108761741 & 0.2259004601200287\end{array}$ $\begin{array}{llll}0.2969351014822088 & 0.5528667067979706 & 0.2154342667121980\end{array}$ $\begin{array}{llll}0.6999646249556122 & 0.5536919969460337 & 0.2154280007541391\end{array}$ $\begin{array}{llll}0.4191722770164842 & 0.6765322560362257 & 0.2005108216618159\end{array}$ $\begin{array}{lllll}0.7003196190922808 & 0.4070318070538814 & 0.2269011532805515\end{array}$ $\begin{array}{llll}0.3244437268258621 & 0.6417258677922902 & 0.2021971341424009\end{array}$ $\begin{array}{llll}0.6695807752047930 & 0.3178809579548319 & 0.2301499776181686\end{array}$ $\begin{array}{lllll}0.4506597427509688 & 0.1931505309327082 & 0.2337390097704753\end{array}$ $\begin{array}{llll}0.5471394322740467 & 0.1934266386227529 & 0.2340204756813473\end{array}$ $\begin{array}{llll}0.1999092290545214 & 0.4350229761103587 & 0.2273966032368006\end{array}$ $\begin{array}{llll}0.7967945005462331 & 0.5283821468346299 & 0.2222999459424083\end{array}$ $\begin{array}{llll}0.5507227101070961 & 0.7715741696236468 & 0.1955060410177900\end{array}$ $\begin{array}{llll}0.4454649175917484 & 0.7715084945667520 & 0.1955969127866974\end{array}$ $\begin{array}{llll}0.1999763506511939 & 0.5268652301367289 & 0.2214908342607176\end{array}$ $\begin{array}{llll}0.7967725884924982 & 0.4368445569350409 & 0.2289003094119531\end{array}$ $\begin{array}{llll}0.2734092265006638 & 0.2653315775379202 & 0.2317165511732783\end{array}$ $\begin{array}{llll}0.7245059952558670 & 0.6946306637663503 & 0.2071837846925776\end{array}$ $\begin{array}{llll}0.7238915231707650 & 0.2670769104354185 & 0.2337877479410471\end{array}$ $\begin{array}{lllll}0.2722600227058021 & 0.6940160867778394 & 0.2073765494192536\end{array}$ $\begin{array}{llll}0.4039574500702852 & 0.1357503608996749 & 0.2358387095789838\end{array}$ $\begin{array}{llll}0.5942280746575984 & 0.1364479242196298 & 0.2366174753986246\end{array}$ $\begin{array}{llll}0.4073117969276070 & 0.8247248157920545 & 0.2103883244152716\end{array}$ $\begin{array}{lllll}0.5892104206020641 & 0.8248289536176191 & 0.2101306366839611\end{array}$ $\begin{array}{llll}0.8562103653679095 & 0.5738339615125385 & 0.2211634732697629\end{array}$ $\begin{array}{llll}0.1399969835383195 & 0.3914178493523928 & 0.2319302217960109\end{array}$ $\begin{array}{llll}0.1405205641742857 & 0.5722921553391668 & 0.2204789560110842\end{array}$ $\begin{array}{llll}0.8565300152241609 & 0.3935411055800204 & 0.2341825368703124\end{array}$ 


\section{FeP-Pt(111) - B-0, vdW-DF-revPBE:}

$\mathrm{Pt} \mathrm{Fe} \mathrm{N} \mathrm{C} \mathrm{H}$

1.00000000000000

$\begin{array}{lll}14.2799999999999994 & 0.0000000000000000 & 0.0000000000000000\end{array}$

$\begin{array}{llll}0.0000000000000000 & 14.8409999999999993 & 0.0000000000000000\end{array}$

$\begin{array}{llll}0.0000000000000000 & 0.0000000000000000 & 35.0000000000000000\end{array}$

$\mathrm{Pt} \mathrm{Fe} \mathrm{N} \mathrm{C}$

$\begin{array}{lllll}90 & 1 & 4 & 20 & 12\end{array}$

Direct

$\begin{array}{llll}0.7999999999999972 & 0.6666669999999968 & 0.0000000000000000\end{array}$

$\begin{array}{llll}0.9000040000000027 & 0.8333319999999986 & 0.0000000000000000\end{array}$

$\begin{array}{llll}0.7999999999999972 & 0.3333330000000032 & 0.0000000000000000\end{array}$

$\begin{array}{llll}0.9000040000000027 & 0.4999990000000025 & 0.0000000000000000\end{array}$

$\begin{array}{llll}0.7999999999999972 & 0.0000000000000000 & 0.0000000000000000\end{array}$

$\begin{array}{llll}0.9000040000000027 & 0.1666650000000018 & 0.0000000000000000\end{array}$

$\begin{array}{llll}0.6000090000000000 & 0.6666669999999968 & 0.0000000000000000\end{array}$

$\begin{array}{lllll}0.7000140000000030 & 0.8333319999999986 & 0.0000000000000000\end{array}$

$\begin{array}{lllll}0.3999999999999986 & 0.6666669999999968 & 0.0000000000000000\end{array}$

$\begin{array}{llll}0.5000039999999970 & 0.8333319999999986 & 0.0000000000000000\end{array}$

$\begin{array}{llll}0.6000090000000000 & 0.3333330000000032 & 0.0000000000000000\end{array}$

$\begin{array}{llll}0.7000140000000030 & 0.4999990000000025 & 0.0000000000000000\end{array}$

$\begin{array}{lllll}0.3999999999999986 & 0.3333330000000032 & 0.0000000000000000\end{array}$

$\begin{array}{llll}0.5000039999999970 & 0.4999990000000025 & 0.0000000000000000\end{array}$

$\begin{array}{llll}0.6000090000000000 & 0.0000000000000000 & 0.0000000000000000\end{array}$

$\begin{array}{llll}0.7000140000000030 & 0.1666650000000018 & 0.0000000000000000\end{array}$

$\begin{array}{lllll}0.3999999999999986 & 0.0000000000000000 & 0.0000000000000000\end{array}$

$\begin{array}{lllll}0.5000039999999970 & 0.1666650000000018 & 0.0000000000000000\end{array}$

$\begin{array}{llll}0.2000090000000014 & 0.6666669999999968 & 0.0000000000000000\end{array}$

$\begin{array}{llll}0.3000139999999973 & 0.8333319999999986 & 0.0000000000000000\end{array}$

$\begin{array}{llll}0.0000000000000000 & 0.6666669999999968 & 0.0000000000000000\end{array}$

$\begin{array}{llll}0.1000039999999984 & 0.8333319999999986 & 0.0000000000000000\end{array}$

$\begin{array}{lllll}0.2000090000000014 & 0.3333330000000032 & 0.0000000000000000\end{array}$

$\begin{array}{llll}0.3000139999999973 & 0.4999990000000025 & 0.0000000000000000\end{array}$

$\begin{array}{llll}0.0000000000000000 & 0.3333330000000032 & 0.0000000000000000\end{array}$

$\begin{array}{lllll}0.1000039999999984 & 0.4999990000000025 & 0.0000000000000000\end{array}$

$\begin{array}{llll}0.2000090000000014 & 0.0000000000000000 & 0.0000000000000000\end{array}$

$\begin{array}{llll}0.3000139999999973 & 0.1666650000000018 & 0.0000000000000000\end{array}$

$\begin{array}{llll}0.0000000000000000 & 0.0000000000000000 & 0.0000000000000000\end{array}$

$\begin{array}{llll}0.1000039999999984 & 0.1666650000000018 & 0.0000000000000000\end{array}$

$\begin{array}{lllll}0.9000040000000027 & 0.7222220000000021 & 0.0666289999999989\end{array}$

$\begin{array}{llll}0.7999999999999972 & 0.8888869999999969 & 0.0666289999999989\end{array}$

$\begin{array}{lllll}0.9000040000000027 & 0.3888880000000015 & 0.0666289999999989\end{array}$

$\begin{array}{llll}0.7999999999999972 & 0.5555540000000008 & 0.0666289999999989\end{array}$

$\begin{array}{llll}0.9000040000000027 & 0.0555549999999982 & 0.0666289999999989\end{array}$

$\begin{array}{lllll}0.7999999999999972 & 0.2222209999999976 & 0.0666289999999989\end{array}$

$\begin{array}{llll}0.6000090000000000 & 0.8888869999999969 & 0.0666289999999989\end{array}$

$\begin{array}{lllll}0.7000140000000030 & 0.7222220000000021 & 0.0666289999999989\end{array}$ 
$\begin{array}{llll}0.5000039999999970 & 0.7222220000000021 & 0.0666289999999989\end{array}$ $\begin{array}{llll}0.3999999999999986 & 0.8888869999999969 & 0.0666289999999989\end{array}$ $\begin{array}{lllll}0.6000090000000000 & 0.5555540000000008 & 0.0666289999999989\end{array}$ $\begin{array}{llll}0.7000140000000030 & 0.3888880000000015 & 0.0666289999999989\end{array}$ $\begin{array}{llll}0.5000039999999970 & 0.3888880000000015 & 0.0666289999999989\end{array}$ $\begin{array}{llll}0.3999999999999986 & 0.5555540000000008 & 0.0666289999999989\end{array}$ $\begin{array}{lllll}0.6000090000000000 & 0.2222209999999976 & 0.0666289999999989\end{array}$ $\begin{array}{llll}0.7000140000000030 & 0.0555549999999982 & 0.0666289999999989\end{array}$ $\begin{array}{lllll}0.5000039999999970 & 0.0555549999999982 & 0.0666289999999989\end{array}$ $\begin{array}{lllll}0.3999999999999986 & 0.2222209999999976 & 0.0666289999999989\end{array}$ $\begin{array}{llll}0.2000090000000014 & 0.8888869999999969 & 0.0666289999999989\end{array}$ $\begin{array}{lllll}0.3000139999999973 & 0.7222220000000021 & 0.0666289999999989\end{array}$ $\begin{array}{lllll}0.1000039999999984 & 0.7222220000000021 & 0.0666289999999989\end{array}$ $\begin{array}{llll}0.0000000000000000 & 0.8888869999999969 & 0.0666289999999989\end{array}$ $\begin{array}{llll}0.2000090000000014 & 0.5555540000000008 & 0.0666289999999989\end{array}$ $\begin{array}{llll}0.3000139999999973 & 0.3888880000000015 & 0.0666289999999989\end{array}$ $\begin{array}{lllll}0.1000039999999984 & 0.3888880000000015 & 0.0666289999999989\end{array}$ $\begin{array}{llll}0.0000000000000000 & 0.5555540000000008 & 0.0666289999999989\end{array}$ $\begin{array}{lllll}0.2000090000000014 & 0.2222209999999976 & 0.0666289999999989\end{array}$ $\begin{array}{llll}0.3000139999999973 & 0.0555549999999982 & 0.0666289999999989\end{array}$ $\begin{array}{lllll}0.1000039999999984 & 0.0555549999999982 & 0.0666289999999989\end{array}$ $\begin{array}{lllll}0.0000000000000000 & 0.2222209999999976 & 0.0666289999999989\end{array}$ $\begin{array}{llll}0.8034011720040849 & 0.7822081721679729 & 0.1334030607237639\end{array}$ $\begin{array}{llll}0.9014306253238271 & 0.9444280099903644 & 0.1345703365748949\end{array}$ $\begin{array}{llll}0.7925023740653926 & 0.4438633939047710 & 0.1390840429592534\end{array}$ $\begin{array}{lllll}0.9038197782702151 & 0.6107025486309987 & 0.1326102975452511\end{array}$ $\begin{array}{llll}0.8029477634229720 & 0.1065005911323725 & 0.1332748308850157\end{array}$ $\begin{array}{llll}0.9029834441889814 & 0.2781611265239651 & 0.1324586310435926\end{array}$ $\begin{array}{llll}0.3996325680015754 & 0.7796449170807407 & 0.1332524166029527\end{array}$ $\begin{array}{llll}0.6014100186478955 & 0.7795760478650496 & 0.1332045527646971\end{array}$ $\begin{array}{llll}0.7000549216203350 & 0.9442731563862022 & 0.1343407924539985\end{array}$ $\begin{array}{llll}0.5003651507733724 & 0.9440904105608573 & 0.1345925253646236\end{array}$ $\begin{array}{llll}0.4037574460303070 & 0.4431792774183880 & 0.1316183470405353\end{array}$ $\begin{array}{llll}0.5968003777742514 & 0.4433631591849884 & 0.1318450816067495\end{array}$ $\begin{array}{llll}0.7084926713016562 & 0.6171839322926060 & 0.1377059581484088\end{array}$ $\begin{array}{llll}0.5007443803225066 & 0.6135407316948026 & 0.1312900393510467\end{array}$ $\begin{array}{llll}0.4001047770562148 & 0.1087274722565936 & 0.1335032378414798\end{array}$ $\begin{array}{llll}0.6001504733769639 & 0.1087764024692736 & 0.1334814599608012\end{array}$ $\begin{array}{llll}0.7060847553596550 & 0.2719654308791585 & 0.1374400108148520\end{array}$ $\begin{array}{llll}0.5002003279387355 & 0.2739979118368573 & 0.1318187662720003\end{array}$ $\begin{array}{llll}0.0006301572189070 & 0.7797417645371346 & 0.1344180725700793\end{array}$ $\begin{array}{llll}0.1977601191084449 & 0.7816977881094112 & 0.1334390015818121\end{array}$ $\begin{array}{llll}0.3008351667891443 & 0.9442114358827983 & 0.1343929520829326\end{array}$ $\begin{array}{llll}0.0997458795504427 & 0.9442841826789500 & 0.1345782133039121\end{array}$ $\begin{array}{llll}0.0005727440371050 & 0.4443792359575323 & 0.1333608243860296\end{array}$ $\begin{array}{llll}0.2084583160995758 & 0.4443670790053740 & 0.1394542828303813\end{array}$ $\begin{array}{llll}0.2933388206734531 & 0.6169631401604990 & 0.1377049620058597\end{array}$ 
$\begin{array}{llll}0.0973510278413570 & 0.6107541377216315 & 0.1327553410085297\end{array}$ $\begin{array}{llll}0.0002386576112612 & 0.1093238554612220 & 0.1345923014334178\end{array}$ $\begin{array}{llll}0.1974631218067409 & 0.1064699149871070 & 0.1332252539133848\end{array}$ $\begin{array}{llll}0.2942007539559455 & 0.2719718488275390 & 0.1375451057290160\end{array}$ $\begin{array}{llll}0.0973922444645585 & 0.2781980001010114 & 0.1325276214210866\end{array}$ $\begin{array}{llll}0.5020598531522914 & 0.4426021339055015 & 0.2057962964177886\end{array}$ $\begin{array}{llll}0.3974105927928077 & 0.5383748864228649 & 0.2187845011048860\end{array}$ $\begin{array}{llll}0.3971108602881511 & 0.3471009561730553 & 0.2189591016663359\end{array}$ $\begin{array}{llll}0.6063409174594707 & 0.3470554980048685 & 0.2189437783475140\end{array}$ $\begin{array}{llll}0.6067574774095077 & 0.5385437773943891 & 0.2187842120639190\end{array}$ $\begin{array}{llll}0.5016160317299981 & 0.2140745294419306 & 0.2286773118683668\end{array}$ $0.50235257468879820 .6718193616060704 \quad 0.2277346628737686$ $\begin{array}{llll}0.5900219743991089 & 0.2539670817059819 & 0.2241923774709833\end{array}$ $\begin{array}{llll}0.4139916342344961 & 0.6316255485736162 & 0.2234456080020196\end{array}$ $\begin{array}{llll}0.6973970814452115 & 0.3568104759777787 & 0.2084366300313997\end{array}$ $\begin{array}{llll}0.4132035805058586 & 0.2538802491778621 & 0.2239790523638466\end{array}$ $\begin{array}{llll}0.3061160089984106 & 0.3570941929719409 & 0.2084920065966051\end{array}$ $\begin{array}{llll}0.5906170429540643 & 0.6318427646179645 & 0.2234995987312630\end{array}$ $\begin{array}{llll}0.3064614225542005 & 0.5286176953151001 & 0.2082935619046618\end{array}$ $\begin{array}{llll}0.6976459666923707 & 0.5283500913578884 & 0.2083234233828612\end{array}$ $\begin{array}{llll}0.2578248263124087 & 0.4428640194389495 & 0.2010087516328892\end{array}$ $\begin{array}{llll}0.7455805948214542 & 0.4425454606053057 & 0.2008361318524208\end{array}$ $\begin{array}{llll}0.6743669932881363 & 0.2052137041326443 & 0.2173155051805112\end{array}$ $\begin{array}{llll}0.7420784480566909 & 0.2683005293608026 & 0.2050177053037310\end{array}$ $\begin{array}{llll}0.3294247430712517 & 0.2050038159632370 & 0.2167267611438829\end{array}$ $\begin{array}{llll}0.6746171628284642 & 0.6805839641820498 & 0.2160885096028803\end{array}$ $\begin{array}{llll}0.3305329249493596 & 0.6806317552925679 & 0.2158975848426148\end{array}$ $\begin{array}{llll}0.2623101670573895 & 0.6172518149846568 & 0.2037645038873829\end{array}$ $\begin{array}{llll}0.2616405897362314 & 0.2685588264208897 & 0.2046263646524640\end{array}$ $\begin{array}{llll}0.7423308047404183 & 0.6169304758049582 & 0.2041005826593941\end{array}$ $\begin{array}{llll}0.5016526432541224 & 0.1408528459199390 & 0.2308244149549763\end{array}$ $\begin{array}{llll}0.5021532542046694 & 0.7451257443442099 & 0.2293925448610656\end{array}$ $\begin{array}{llll}0.8185383882804846 & 0.4427206084493880 & 0.2107021465212111\end{array}$ $\begin{array}{llll}0.1851107834397284 & 0.4430814235806070 & 0.2111863695680151\end{array}$ $\begin{array}{llll}0.6835820368349572 & 0.1330030251674952 & 0.2198082918110291\end{array}$ $\begin{array}{llll}0.8172411071242620 & 0.2575710529720631 & 0.2042664357217635\end{array}$ $\begin{array}{llll}0.1871177543356450 & 0.6282315108709127 & 0.2041064834292369\end{array}$ $\begin{array}{llll}0.3215212484852081 & 0.7528606378652327 & 0.2183855048025230\end{array}$ $\begin{array}{llll}0.6840143435751631 & 0.7528136362512863 & 0.2184242146075951\end{array}$ $\begin{array}{llll}0.3202277165165637 & 0.1327552550535958 & 0.2191724312361690\end{array}$ $\begin{array}{llll}0.1864615101210044 & 0.2578161617055038 & 0.2041452658474539\end{array}$ $\begin{array}{llll}0.8175742403047748 & 0.6274688293832552 & 0.2040971353510176\end{array}$

\section{FeP-Pt(111) - B-45, vdW-DF-revPBE:}

$\mathrm{Pt} \mathrm{Fe} \mathrm{N} \mathrm{C} \mathrm{H}$ 1.00000000000000 $\begin{array}{lll}14.2799999999999994 & 0.0000000000000000 & 0.0000000000000000\end{array}$ 


$\begin{array}{rrrr}0.0000000000000000 & 14.84099999999999993 & 0.0000000000000000 \\ 0.0000000000000000 & 0.0000000000000000 & 35.0000000000000000 \\ \text { Pt Fe N C H } & & \end{array}$

$\begin{array}{lllll}90 & 1 & 4 & 20 & 12\end{array}$

Direct

$\begin{array}{llll}0.7999999999999972 & 0.6666669999999968 & 0.0000000000000000\end{array}$

$\begin{array}{lllll}0.9000040000000027 & 0.8333319999999986 & 0.00000000000000000\end{array}$

$\begin{array}{llll}0.7999999999999972 & 0.3333330000000032 & 0.00000000000000000\end{array}$

$\begin{array}{lllll}0.9000040000000027 & 0.4999990000000025 & 0.0000000000000000\end{array}$

$\begin{array}{lllll}0.7999999999999972 & 0.0000000000000000 & 0.0000000000000000\end{array}$

$\begin{array}{lllll}0.9000040000000027 & 0.1666650000000018 & 0.0000000000000000\end{array}$

$\begin{array}{lllll}0.6000090000000000 & 0.6666669999999968 & 0.0000000000000000\end{array}$

$\begin{array}{lllll}0.7000140000000030 & 0.8333319999999986 & 0.0000000000000000\end{array}$

$\begin{array}{lllll}0.3999999999999986 & 0.6666669999999968 & 0.0000000000000000\end{array}$

$\begin{array}{lllll}0.5000039999999970 & 0.8333319999999986 & 0.0000000000000000\end{array}$

$\begin{array}{lllll}0.6000090000000000 & 0.33333330000000032 & 0.0000000000000000\end{array}$

$\begin{array}{llll}0.7000140000000030 & 0.4999990000000025 & 0.0000000000000000\end{array}$

$\begin{array}{lllll}0.3999999999999986 & 0.33333330000000032 & 0.0000000000000000\end{array}$

$\begin{array}{lllll}0.5000039999999970 & 0.4999990000000025 & 0.0000000000000000\end{array}$

$\begin{array}{lllll}0.6000090000000000 & 0.0000000000000000 & 0.0000000000000000\end{array}$

$\begin{array}{lllll}0.7000140000000030 & 0.1666650000000018 & 0.0000000000000000\end{array}$

$\begin{array}{lllll}0.3999999999999986 & 0.0000000000000000 & 0.0000000000000000\end{array}$

$\begin{array}{llllll}0.5000039999999970 & 0.1666650000000018 & 0.0000000000000000\end{array}$

$\begin{array}{llllll}0.2000090000000014 & 0.6666669999999968 & 0.0000000000000000\end{array}$

$\begin{array}{lllll}0.3000139999999973 & 0.8333319999999986 & 0.0000000000000000\end{array}$

$\begin{array}{lllll}0.0000000000000000 & 0.6666669999999968 & 0.0000000000000000\end{array}$

$\begin{array}{lllll}0.1000039999999984 & 0.8333319999999986 & 0.0000000000000000\end{array}$

$\begin{array}{llllll}0.2000090000000014 & 0.3333330000000032 & 0.0000000000000000\end{array}$

$\begin{array}{lllll}0.3000139999999973 & 0.4999990000000025 & 0.0000000000000000\end{array}$

$\begin{array}{lllll}0.0000000000000000 & 0.3333330000000032 & 0.0000000000000000\end{array}$

$\begin{array}{lllll}0.1000039999999984 & 0.4999990000000025 & 0.0000000000000000\end{array}$

$\begin{array}{lllll}0.2000090000000014 & 0.0000000000000000 & 0.0000000000000000\end{array}$

$\begin{array}{lllll}0.3000139999999973 & 0.1666650000000018 & 0.0000000000000000\end{array}$

$\begin{array}{lllll}0.0000000000000000 & 0.0000000000000000 & 0.0000000000000000\end{array}$

$\begin{array}{lllll}0.1000039999999984 & 0.1666650000000018 & 0.0000000000000000\end{array}$

$\begin{array}{lllll}0.9000040000000027 & 0.7222220000000021 & 0.06662899999999989\end{array}$

$\begin{array}{lllll}0.7999999999999972 & 0.8888869999999969 & 0.0666289999999989\end{array}$

$\begin{array}{lllll}0.9000040000000027 & 0.3888880000000015 & 0.0666289999999989\end{array}$

$\begin{array}{lllll}0.7999999999999972 & 0.5555540000000008 & 0.0666289999999989\end{array}$

$\begin{array}{llllll}0.9000040000000027 & 0.0555549999999982 & 0.0666289999999989\end{array}$

$\begin{array}{lllll}0.7999999999999972 & 0.2222209999999976 & 0.0666289999999989\end{array}$

$\begin{array}{llllll}0.6000090000000000 & 0.8888869999999969 & 0.0666289999999989\end{array}$

$\begin{array}{lllll}0.7000140000000030 & 0.7222220000000021 & 0.0666289999999989\end{array}$

$\begin{array}{lllll}0.5000039999999970 & 0.7222220000000021 & 0.0666289999999989\end{array}$

$\begin{array}{lllll}0.3999999999999986 & 0.8888869999999969 & 0.0666289999999989\end{array}$

$\begin{array}{lllll}0.6000090000000000 & 0.5555540000000008 & 0.0666289999999989\end{array}$

$\begin{array}{lllll}0.7000140000000030 & 0.3888880000000015 & 0.0666289999999989\end{array}$ 
$\begin{array}{llll}0.5000039999999970 & 0.3888880000000015 & 0.0666289999999989\end{array}$ $\begin{array}{llll}0.3999999999999986 & 0.5555540000000008 & 0.0666289999999989\end{array}$ $\begin{array}{lllll}0.6000090000000000 & 0.2222209999999976 & 0.0666289999999989\end{array}$ $\begin{array}{llll}0.7000140000000030 & 0.0555549999999982 & 0.0666289999999989\end{array}$ $\begin{array}{lllll}0.5000039999999970 & 0.0555549999999982 & 0.0666289999999989\end{array}$ $\begin{array}{lllll}0.3999999999999986 & 0.2222209999999976 & 0.0666289999999989\end{array}$ $\begin{array}{llll}0.2000090000000014 & 0.8888869999999969 & 0.0666289999999989\end{array}$ $\begin{array}{llll}0.3000139999999973 & 0.7222220000000021 & 0.0666289999999989\end{array}$ $\begin{array}{lllll}0.1000039999999984 & 0.7222220000000021 & 0.0666289999999989\end{array}$ $\begin{array}{llll}0.0000000000000000 & 0.8888869999999969 & 0.0666289999999989\end{array}$ $\begin{array}{llll}0.2000090000000014 & 0.5555540000000008 & 0.0666289999999989\end{array}$ $\begin{array}{llll}0.3000139999999973 & 0.3888880000000015 & 0.0666289999999989\end{array}$ $\begin{array}{lllll}0.1000039999999984 & 0.3888880000000015 & 0.0666289999999989\end{array}$ $\begin{array}{llll}0.0000000000000000 & 0.5555540000000008 & 0.0666289999999989\end{array}$ $\begin{array}{lllll}0.2000090000000014 & 0.2222209999999976 & 0.0666289999999989\end{array}$ $\begin{array}{llll}0.3000139999999973 & 0.0555549999999982 & 0.0666289999999989\end{array}$ $\begin{array}{lllll}0.1000039999999984 & 0.0555549999999982 & 0.0666289999999989\end{array}$ $\begin{array}{lllll}0.0000000000000000 & 0.2222209999999976 & 0.0666289999999989\end{array}$ $\begin{array}{llll}0.8023351196654148 & 0.7797429506697398 & 0.1336032109610950\end{array}$ $\begin{array}{llll}0.9018540795773583 & 0.9449367691930739 & 0.1342586831314500\end{array}$ $\begin{array}{llll}0.8050055262986350 & 0.4450218524168383 & 0.1327532919975566\end{array}$ $\begin{array}{llll}0.9019235872335734 & 0.6129213552678999 & 0.1337204197278150\end{array}$ $\begin{array}{llll}0.8022436607381314 & 0.1106545737043395 & 0.1336438574796227\end{array}$ $\begin{array}{llll}0.9020924390732987 & 0.2768353978699528 & 0.1340573830884362\end{array}$ $\begin{array}{llll}0.4026255388368427 & 0.7784018714758052 & 0.1382948409362436\end{array}$ $\begin{array}{lllll}0.6004589182026474 & 0.7779999412482393 & 0.1380842467572440\end{array}$ $\begin{array}{llll}0.7016759689860287 & 0.9455847250986336 & 0.1335595881104820\end{array}$ $\begin{array}{llll}0.5015376004499705 & 0.9458445625139612 & 0.1327274936392894\end{array}$ $\begin{array}{llll}0.3966320013695719 & 0.4452568530465449 & 0.1319804659340885\end{array}$ $\begin{array}{llll}0.6038451665438218 & 0.4447473837438958 & 0.1320187477951506\end{array}$ $\begin{array}{llll}0.7023266614293948 & 0.6137222031835582 & 0.1379701612759063\end{array}$ $\begin{array}{llll}0.5008779580697350 & 0.6071334404535900 & 0.1291992911237475\end{array}$ $\begin{array}{lllll}0.4029749942016091 & 0.1123589272978071 & 0.1387618282556243\end{array}$ $\begin{array}{llll}0.6012128997050743 & 0.1126672166154863 & 0.1386115467863045\end{array}$ $\begin{array}{llll}0.7026949279708132 & 0.2775902674199190 & 0.1377068630853369\end{array}$ $\begin{array}{lllll}0.5003065289237694 & 0.2811592186546842 & 0.1290000073600455\end{array}$ $\begin{array}{llll}0.0009706521454011 & 0.7792111762256803 & 0.1342758297118961\end{array}$ $\begin{array}{llll}0.1996130585641270 & 0.7798399943705400 & 0.1337178840752236\end{array}$ $\begin{array}{llll}0.3011130851717246 & 0.9455963795672064 & 0.1335423556686657\end{array}$ $\begin{array}{lllll}0.1006570963388160 & 0.9451223352597879 & 0.1342405929194928\end{array}$ $\begin{array}{lllll}0.0010600962576966 & 0.4451903452000964 & 0.1340017781601213\end{array}$ $\begin{array}{llll}0.1967204577359025 & 0.4462181016932250 & 0.1327414239307441\end{array}$ $\begin{array}{llll}0.2995503883809046 & 0.6147407579983205 & 0.1380817718897603\end{array}$ $\begin{array}{llll}0.0998786294296446 & 0.6133297457773521 & 0.1339814803873364\end{array}$ $\begin{array}{lllll}0.0013263745090756 & 0.1111121444950770 & 0.1341006168288580\end{array}$ $\begin{array}{llll}0.2003307958556275 & 0.1106314544081167 & 0.1334915610151733\end{array}$ $\begin{array}{llll}0.2994987272580281 & 0.2773673377438368 & 0.1378048291513416\end{array}$ 
$\begin{array}{llll}0.1001094741213478 & 0.2770003550964111 & 0.1339951923484018\end{array}$

$\begin{array}{llll}0.5041452317480370 & 0.4455809054409645 & 0.1983845994118122\end{array}$

$\begin{array}{llll}0.3615232920342729 & 0.4464778226025912 & 0.2116551797989538\end{array}$

$\begin{array}{llll}0.5029857468548599 & 0.2969661141169314 & 0.2067987658856220\end{array}$

$\begin{array}{llll}0.6453432031887263 & 0.4465524270292514 & 0.2128953367198009\end{array}$

$\begin{array}{llll}0.5029925781197641 & 0.5956160317601427 & 0.2061660205944678\end{array}$

$\begin{array}{llll}0.6757964021860057 & 0.2796833835671059 & 0.2062197395802902\end{array}$

$\begin{array}{lllll}0.3301114745435715 & 0.6132409937055108 & 0.2047326272189420\end{array}$

$\begin{array}{llll}0.7028259577804062 & 0.3709917321630368 & 0.2154987912973882\end{array}$

$\begin{array}{llll}0.3038324750864348 & 0.5217904223343786 & 0.2145085335452317\end{array}$

$\begin{array}{llll}0.7029433054578250 & 0.5218092852039276 & 0.2149505257365013\end{array}$

$\begin{array}{llll}0.5819137619898956 & 0.2437474679709789 & 0.2043328196800679\end{array}$

$\begin{array}{llll}0.4244569731567679 & 0.2436948145406959 & 0.2033250572810812\end{array}$

$\begin{array}{llll}0.4244073532877161 & 0.6490382041023338 & 0.2031398655693415\end{array}$

$\begin{array}{llll}0.3039673651632398 & 0.3709279452537235 & 0.2146880068575105\end{array}$

$\begin{array}{llll}0.5819082350886153 & 0.6490248032745400 & 0.2035681633691506\end{array}$

$\begin{array}{llll}0.3302511363255117 & 0.2796129523749246 & 0.2048214696702114\end{array}$

$\begin{array}{llll}0.6760768569038839 & 0.6132377090307827 & 0.2053445008769922\end{array}$

$\begin{array}{llll}0.7979961257214817 & 0.3995366174522215 & 0.2212006188357366\end{array}$

$\begin{array}{llll}0.7980829133360786 & 0.4934157515536125 & 0.2209114007167866\end{array}$

$\begin{array}{llll}0.5562602602515484 & 0.1479693698973105 & 0.1966994191455606\end{array}$

$\begin{array}{llll}0.4499726052852832 & 0.7450144148471318 & 0.1963612238903308\end{array}$

$\begin{array}{lllll}0.2092380562089697 & 0.4932690664467077 & 0.2211635671387898\end{array}$

$\begin{array}{llll}0.2093443481319608 & 0.3993042001540102 & 0.2213145278543618\end{array}$

$\begin{array}{llll}0.4500866780753593 & 0.1476637984749556 & 0.1965541094312004\end{array}$

$\begin{array}{llll}0.5560795691252319 & 0.7448532716965812 & 0.1964660353349762\end{array}$

$\begin{array}{llll}0.7298331328941747 & 0.2293757099156988 & 0.2116298684397236\end{array}$

$\begin{array}{llll}0.2767815855437617 & 0.6636317845742435 & 0.2112668556171447\end{array}$

$\begin{array}{llll}0.7296777749013659 & 0.6636897830381551 & 0.2112899224630560\end{array}$

$\begin{array}{llll}0.2766885735823464 & 0.2293298512385172 & 0.2111334575646447\end{array}$

$\begin{array}{lllll}0.8581073017669073 & 0.3552940289911908 & 0.2236510822983045\end{array}$

$\begin{array}{lllll}0.8581815397020452 & 0.5377700917840001 & 0.2231519956980746\end{array}$

$\begin{array}{llll}0.1494184199621512 & 0.3550648988800574 & 0.2242668812474410\end{array}$

$\begin{array}{llll}0.1492477009651993 & 0.5374762575577876 & 0.2240781542628363\end{array}$

$\begin{array}{llll}0.4133767674869500 & 0.7967861066939039 & 0.2128576214347496\end{array}$

$\begin{array}{llll}0.5924838290030318 & 0.0957290947842799 & 0.2130786559880778\end{array}$

$\begin{array}{lllll}0.4135965995292831 & 0.0960031576830761 & 0.2131733360572610\end{array}$

$\begin{array}{llll}0.5924732510452834 & 0.7969751813088056 & 0.2128242833259213\end{array}$ 